\title{
The Use of Ultraviolet Thomson Scattering as a Versatile Diagnostic for Detailed Measurements of a Collisional Laser Produced Plasma
}

Mark David Tracy

(Ph.D. Thesis)

January 8, 1993

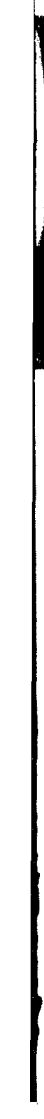


This document was prepared as an account of work sponsored by an agency of the Lnited States Government. Neither the Lnited States Government nor the Lniversity of California nor any of their employees, makes any warranty, express or implied, or assumes any legal liability or responsibility for the accuracy, completeness. or usefulness of any information, apparatus, product, or process disclosed, or represents that its use would not infringe privately owned rights. Reference herein to any specific commercial products, process, or service by trade name, trademark, manufacturer, or otherwise, does not necessarily constitute or imply its endorsement. recommendation, or favoring by the Lnited States Government or the Lniversity of California. The views and opinions of authors expressed herein du not necessarily state or reflect those of the Linited States Government or the Lniversity of Califurnia, and shall not be used for advertising or product endorsement purposes.

This report has been reproduced directly from the best available copy.

A vailable to DOE and DOE contractors from the Office of Scientific and Technical Information

P.O. Box 62, Oak Ridge, TN 37831

Prices available from (615) 576.8401, FTS 626-8401

Available to the public from the National Technical Information Service

L.S. Department of Commerce 5285 Port Royal Rd.,

Springfield, VA 22161

Work performed under the auspices of the L.S. Department of Energy by Lawrence Livermore National Laboratory under Contract W-7405-Eng-48. 
The Use of Ultraviolet Thomson Scattering as a Versatile Diagnostic for Detailed Measurements of a Collisional Laser Produced Plasma

\author{
Mark David Tracy \\ (Ph.D. Thesis)
}

Manuscript date: January 8, 1993

\title{
LAWRENCE LIVERMORE NATIONAL LABORATORY
} University of California - Livermore, California • 94551

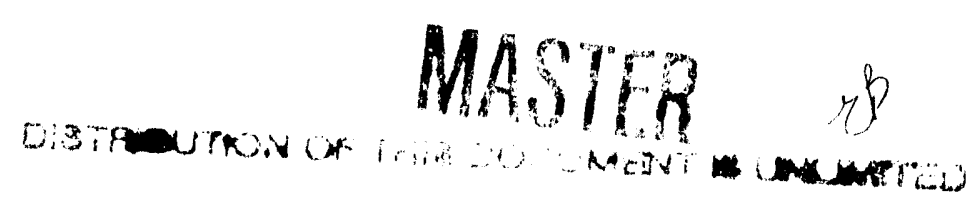


The Use of Ultraviolet Thomson Scattering as a Versatile Diagnostic for Detailed Measurements of a Collisional Laser Produced Plasma

By

Mark David Tracy

B.S. Physics (University of Rochester) 1987

B.S. Optics (University of Rochester) 1987

M.S. Engineering and Applied Science (University of California, Davis) 1988

\section{DISSERTATION}

Submitted in partial satisfaction of the requirements for the degree of

DOCTOR OF PHILOSOPHY
in
ENGINEERING-APPLIED SCIENCE
in the
GRADUATE DIVISION
of the
UNIVERSITY OF CALIFORNIA
DAVIS

Approved:

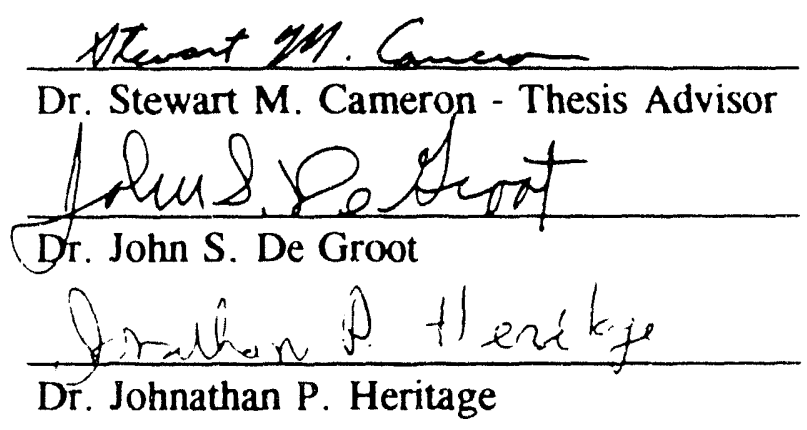

Committee in Charge

1993

$-\mathrm{i}-$ 


\section{Copyright by}

\section{Mark David Tracy}

1993

The Goverment reserves for itself and others acting on its behalf a royalty free, nonexclusive, irrevocable, world-wide license for govermental purposes to publish, distribute, translate, duplicate, exhibit, and perform any such data copyrighted by the contractor. 
There are many individuals I would like to extend my appreciation to for their help and guidance, which they often gave above and beyond the call of duty. I extend my appreciation to Dr. David Milam for always being able to find the right optic for me when desperation struck and Dr. Ian Thomas whose wizardry with Sol Gel coatings made much of my experiment possible. I would like to thank Dr. Edward Williams for his never ending supply of answers to any question ever posed to him. I am grateful to Dr. John De Groot both for his moral and scientific support in my thesis. A resounding applause is given for Dr. Kent Estabrook. I will always be indebted to him for always making time to listen to my scientific rambling and for his sometimes constant computational support on the CRAY's. I would like to thank my advisor, Dr. Stewart Cameron, for the support and motivation for completing my thesis project. Finally, to the soon to be Dr. Christopher Bonang, thank you for keeping my stay as a graduate student in the laboratory a joyous one.

I wish to acknowledge the financial support of the Fannie and John Hertz Foundation which eased the burden of graduate school.

This thesis is dedicated to my loving wife Julia and my wonderful son Quinn who have made surviving the graduate school experience in Livermore, California possible. 
Collective Thomson scattering from ion-acoustic waves at $266 \mathrm{~nm}$ is used to obtain spatially resolved, two-dimensional electron density, sound speed, and radial drift profiles of a collisional laser plasma (critical density, $\mathrm{n}_{\mathrm{c}}=1 \times 10^{21} \mathrm{~cm}^{-3}$ ). An ultraviolet diagnostic wavelength minimizes the complicating effects of inverse bremsstrahlung and refractive turning in the coronal region of interest, where the electron densities approach $n_{c} / 10$. Laser plasmas of this type are important because they model some of the aspects of the plasmas found in high-gain laser-fusion pellets irradiated by long pulse widths $\left(t_{L}\right.$ $\geq 10 \mathrm{nsec}$ ), where the laser light is absorbed mostly in the corona. The experimental results and LASNEX [Comments Plasma Phys. Controlled Fusion 2, 51 (1975)] simulations agree within a percent standard deviation of $40 \%$ for the electron density and $50 \%$ for the sound speed and radial drift velocity. Thus it is shown that the hydrodynamics equations with classical coefficients and the numerical approximations in LASNEX are valid models of laser-heated, highly collisional plasmas.

The versatility of Thomson scattering is expanded upon by extending existing theory with a Fokker-Planck based model to include plasmas that are characterized by ( 0 $\left.\leq \mathrm{k}_{\mathrm{ia}} \lambda_{\mathrm{ii}} \leq \infty\right)$ and $\mathrm{ZT}_{\mathrm{e}} / \mathrm{T}_{\mathrm{i}}$, where $\mathrm{k}_{\mathrm{ia}}$ is the ion-acoustic wave number, $\lambda_{\mathrm{ii}}$ is the ion-ion mean free path, $\mathrm{Z}$ is the ionization state of the plasma, and $\mathrm{T}_{\mathrm{e}}, \mathrm{T}_{\mathrm{i}}$ are the electron and ion temperatures in elcctron volts (eV), respectively. The model is valid for plasmas in which the electrons are approximately collisionless, $\left(\mathrm{k}_{\mathrm{ia}} \lambda_{\mathrm{ei}}, \mathrm{k}_{\mathrm{ia}} \lambda_{\mathrm{ee}} \geq 1\right)$, and quasineutrality holds, $(\alpha * 1)$, where $\alpha=1 / \mathrm{k} \lambda_{\mathrm{DE}}$ and $\lambda_{\mathrm{DE}}$ is the electron Debye length. 
This newly developed model predicts the lineshape of the ion-acoustic Thomson spectra and when fit to experimental data provides a direct measurement of the relative thermal flow velocity between the electrons and ions. The model also correctly predicts the appearance of a zero-frequency or entropy peak in the Thomson spectra in the ion-ion collisional limit. 
Table of Contents

Chapter I: Laser-produced plasma diagnostics $\ldots \ldots \ldots \ldots \ldots$

Laser-produced plasmas .................... 2

Electric $(\mathrm{E})$ and magnetic fields $(\mathrm{B}) \ldots \ldots \ldots \ldots$

Refractive index .................. 6

Electromagnetic absorption and radiation $\ldots \ldots \ldots . \ldots 7$

Self-emissive diagnostics . . . . . . . . . . . . . . . 9

Refractive/absorptive diagnostics . . . . . . . . . . . . . 13

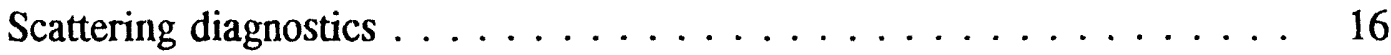

Choosing a diagnostic . . . . . . . . . . . . . . . . 19

Collective Thomson scattering ................ 20

Dissertation organization ...................... 22

Chapter II: Thomson scattering theory - fluid and kinetic limits . . . . . . . 24

General scattered light conditions ................ 25

Generalized dynamical form factor $\mathbf{S}(\mathbf{k}, \boldsymbol{\omega}) \ldots \ldots \ldots 26$

The fluctuation-dissipation theorem . . . . . . . . . 27

Coulomb's law ...................... 28

The continuity equation $\ldots \ldots \ldots \ldots \ldots \ldots$

Ohm's law ................................ 29

Generalized electron and ion susceptibilities ............ 32 
High frequency limit $(\omega \rightarrow \infty) \quad \ldots \ldots \ldots \ldots \ldots \ldots$

D.C. limit $(\omega \rightarrow 0) \quad \ldots \ldots \ldots \ldots \ldots \ldots \ldots \ldots \ldots \ldots$

Generalized total cross section $\mathrm{S}_{\mathrm{T}}(\mathbf{k}) \ldots \ldots \ldots \ldots \ldots \ldots \ldots$

Dynamical form factor (collisionless limit) $\ldots \ldots \ldots \ldots \ldots \ldots . . \ldots$

Spectral characteristics $\ldots \ldots \ldots \ldots \ldots \ldots \ldots \ldots$

Peak height asymmetry $\ldots \ldots \ldots \ldots \ldots \ldots \ldots \ldots \ldots 42$

Dynamical form factor (collisional limit) $\ldots \ldots \ldots \ldots \ldots \ldots \ldots 44$

Measuring the three quantities $\ldots \ldots \ldots \ldots \ldots \ldots \ldots \ldots$

Electron density $\ldots \ldots \ldots \ldots \ldots \ldots \ldots \ldots \ldots$

Sound speed . . . . . . . . . . . . . . . . 49

Radial drift $\ldots \ldots \ldots \ldots \ldots \ldots \ldots \ldots \ldots \ldots$

Chapter III: Experimental configuration $\ldots \ldots \ldots \ldots \ldots \ldots \ldots \ldots . \ldots \ldots$

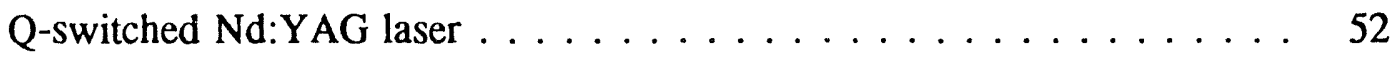

Pulse-to-pulse energy stability $\ldots \ldots \ldots \ldots \ldots \ldots \ldots \ldots 2$

Injection seeding $\ldots \ldots \ldots \ldots \ldots \ldots \ldots \ldots \ldots$

Variable reflectivity mirror $\ldots \ldots \ldots \ldots \ldots \ldots \ldots$

Plasma generation $\ldots \ldots \ldots \ldots \ldots \ldots \ldots \ldots \ldots \ldots$

Plasma coordinate system $\ldots \ldots \ldots \ldots \ldots \ldots \ldots \ldots$

Ultraviolet diagnostic beam $\ldots \ldots \ldots \ldots \ldots \ldots \ldots$

Thomson scattering collection optics $\ldots \ldots \ldots \ldots \ldots \ldots$

Stray light reduction $\ldots \ldots \ldots \ldots \ldots \ldots \ldots \ldots \ldots \ldots$ 
Timing system $\ldots \ldots \ldots \ldots \ldots \ldots \ldots \ldots \ldots \ldots \ldots$

Rough alignment of the diagnostic beam $\ldots \ldots \ldots \ldots \ldots 6$

Target debris $\ldots \ldots \ldots \ldots \ldots \ldots \ldots \ldots \ldots \ldots \ldots$

Chapter IV: Data acquisition and analysis . . . . . . . . . . . . 69

Collective Thomson spectra $\ldots \ldots \ldots \ldots \ldots \ldots \ldots \ldots$

Bremsstrahlung emission from the plasma $\ldots \ldots \ldots \ldots \ldots 71$

Background subtraction $\ldots \ldots \ldots \ldots \ldots \ldots \ldots \ldots \ldots$

Measurement of electron density $\ldots \ldots \ldots \ldots \ldots \ldots \ldots \ldots$

$\boldsymbol{B}_{\text {adj }}$ parameter $\ldots \ldots \ldots \ldots \ldots \ldots \ldots \ldots \ldots \ldots \ldots$

$\mathrm{S}_{\mathrm{T}}{ }^{\mathrm{i}}\left(\mathbf{k}_{\mathrm{ia}}\right)$ and $\mathrm{e}^{-\Delta}$ parameters $\ldots \ldots \ldots \ldots \ldots \ldots \ldots$

Rayleigh calibration of the collection system $\ldots \ldots \ldots \ldots 79$

Measurement of the sound speed and radial drift $\ldots \ldots \ldots \ldots 2$

Absolute distances $\ldots \ldots \ldots \ldots \ldots \ldots \ldots \ldots \ldots$

Plasma reproducibility $\ldots \ldots \ldots \ldots \ldots \ldots \ldots \ldots \ldots$

Nonintrusive diagnostic beam $\ldots \ldots \ldots \ldots \ldots \ldots . \ldots . \ldots 8$

Results from the collective Thomson scattering measurements . . . . . 89

Physical modeling $\ldots \ldots \ldots \ldots \ldots \ldots \ldots \ldots \ldots$

$\mathrm{Z}$ and $\mathrm{T}_{\mathrm{e}}$ from equilibrium models $\ldots \ldots \ldots \ldots \ldots$

Radial sonic point from steady state equation of motion $\ldots \ldots 97$

Comparison of experimental data with LASNEX . . . . . . . . 100

Simple analytical model $\ldots \ldots \ldots \ldots \ldots \ldots$ 
Answers to questions . . . . . . . . . . . . . . . . 108

Are electron and ion temperatures equal? . . . . . . . . . 108

Is a 532nm diagnostic wavelength suitable? . . . . . . . . . . 109

Inconsistent peak height asymmetries $\ldots \ldots \ldots \ldots \ldots \ldots$

Chapter V: Thomson scattering theory - intermediate ion-ion collisional regime . 116

General closure relations . . . . . . . . . . . . . . . 118

Modified ion susceptibility $\ldots \ldots \ldots \ldots \ldots \ldots$

Modified dynamical form factor $\mathbf{S}(\mathbf{k}, \boldsymbol{\omega}) \ldots \ldots \ldots \ldots$

Modified fluid model in collisionless Landau limit . . . . . . . . . . . . 122

Perturbed plasma quantities $n_{1}, u_{1}, T_{1}, q_{1}$, and $\pi_{1} \ldots \ldots 122$

Transport coefficients ... . . . . . . . . . . . . 124

Ion Landau damping . . . . . . . . . . . . . . . . . . . 124

Ion-acoustic spectrum $\ldots \ldots \ldots \ldots \ldots \ldots \ldots$

Solution of the Fokker-Planck equation . . . . . . . . . . . . . 127

The Fokker-Planck equation . . . . . . . . . . . . . 128

Linearized Fokker-Planck equation . . . . . . . . . . . . . . 129

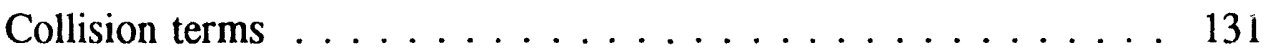

Perturbed ion distribution function $\ldots \ldots \ldots 131$

Matrix form of the solution $\ldots \ldots \ldots 133$

Entropy wave results $\ldots \ldots \ldots \ldots 135$

Ion-acoustic wave results $\ldots \ldots \ldots \ldots 141$ 
Calculation of $\kappa_{1}, \kappa_{2}, \mu_{1}, \mu_{2}$ and $\Gamma \ldots \ldots \ldots \ldots \ldots \ldots$

Self-consistency of $\kappa_{1}, \kappa_{2}, \mu_{1}, \mu_{2}$, and $\Gamma \ldots \ldots \ldots \ldots \ldots \ldots$

Peak height asymmetry $\ldots \ldots \ldots \ldots \ldots \ldots \ldots \ldots \ldots$

Application of the modified fluid model $\ldots \ldots \ldots \ldots \ldots \ldots$

Chapter VI: Experimental configuration Il (peak asymmetry investigation) $\ldots . .151$

The new CCD detector $\ldots \ldots \ldots \ldots \ldots \ldots \ldots \ldots \ldots \ldots$

Modification of collection system $\ldots \ldots \ldots \ldots \ldots \ldots \ldots$

Astigmatic compensation of the spectrometer $\ldots \ldots \ldots \ldots \ldots 157$

Chapter VII: Conclusions $\ldots \ldots \ldots \ldots \ldots \ldots$. . . . . . . . . 164

Appendix: Code for eigenvalue solution of the Fokker-Planck $\ldots \ldots \ldots$. . 169




Figures

Figure 1: Total cross section $\mathrm{S}_{\mathrm{T}}(\mathbf{k})$ for a general plasma as a function of $\mathrm{ZT}_{\mathrm{e}} / \mathrm{T}_{\mathrm{i}}$



Figure 2: Real and imaginary parts of the complex ion-acoustic frequency as a function of $\mathrm{ZT}_{\mathrm{e}} / \mathrm{T}_{\mathrm{i}}$ for a collisionless plasma with $\alpha \rightarrow \infty$ neglecting electron Landau damping. . . . . . . . . . . . . . . . . . . . 39

Figure 3: Real and imaginary parts of the function $W(x) . \ldots \ldots \ldots$

Figure 4: Theoretical ion-acoustic Thomson spectra for a collisionless aluminum plasma with $\mathrm{ZT}_{e} / \mathrm{T}_{\mathrm{i}}=4$ and varying values of $\alpha . \ldots \ldots \ldots$

Figure 5: Theoretical ion-acoustic Thomson spectra for a collisionless aluminum plasma with $\alpha=1$ and varying values of $\mathrm{ZT}_{\mathrm{e}} / \mathrm{T}_{\mathrm{i}} \ldots \ldots \ldots$

Figure 6: Peak height asymmetries due to a relative drift between electrons and ions in an aluminum plasma with $\mathrm{ZT}_{\mathrm{e}} / \mathrm{T}_{\mathrm{i}}=8, \alpha=3$, and different values

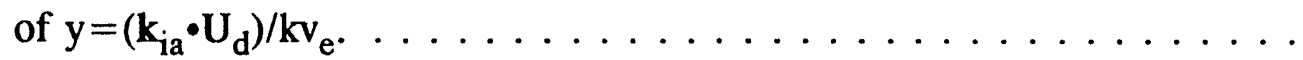

Figure 7: Theoretical ion-acoustic Thomson spectra in the ion fluid $\left(\mathrm{k}_{\mathrm{ia}} \lambda_{\mathrm{ii}}=0.1\right)$ and ion collisionless limits for a plasma with $\mathrm{ZT}_{\mathrm{e}} / \mathrm{T}_{\mathrm{i}}=1$ and $\alpha=300$. 46

Figure 8: Total ion cross section $S_{\mathrm{T}}{ }^{\mathrm{i}}(\mathbf{k})$ in the collisionless (line) and fluid (circle) ion limits as a function of $\alpha$ for $\mathrm{ZT}_{\mathrm{e}} / \mathrm{T}_{\mathrm{i}}$ ratios $(1 \rightarrow 8)$ in an aluminum

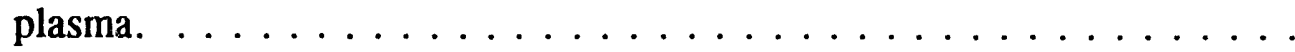

Figure 9: Experimental configuration; top view. . . . . . . . . . . 52

Figure 10: Experimental configuration; side view. . . . . . . . . . 53 
Figure 11: Experimental configuration; side view of target. . . . . . . . 54

Figure 12: Temporal profile of the Nd:YAG laser pulse during unseeded

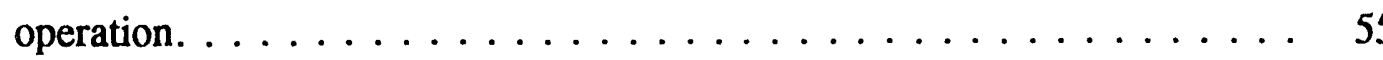

Figure 13: Temporal profile of the Nd:YAG laser pulse during seeded operation (solid line) with a Gaussian pulse shape of the same (FWHM $=9 \mathrm{nsec})$ width for comparison (dashed line) $\ldots \ldots \ldots \ldots \ldots \ldots$

Figure 14: Near-field spatial profile of the $1.06 \mu \mathrm{m}$ pulse produced by a Qswitched, Nd:YAG laser equipped with a hard edged output coupler. . . .

Figure 15: Near-field spatial profile of the $1.06 \mu \mathrm{m}$ pulse produced by a Qswitched, Nd:YAG laser equipped with a variable reflectivity mirror (solid line). The best fit Gaussian (dashed line) is included (FWHM width $8.2 \mathrm{~mm}) . \ldots \ldots \ldots \ldots \ldots \ldots \ldots \ldots \ldots \ldots$

Figure 16: Focused $1.06 \mu \mathrm{m}$ beam at the target surface (circles) with a best fit Gaussian profile (dashed line). The best fit Gaussian to the data has a width $(\mathrm{FWHM}=92 \pm 10 \mu \mathrm{m}) . \ldots \ldots \ldots \ldots \ldots \ldots$

Figure 17: Optical schematic of the collection system which couples the Thomson scattered signal from the plasma into the spectrometer. . . . . . . . 61

Figure 18: Timing schematic for the Thomson scattering experimental configuration.

Figure 19: Raw data of collective Thomson scattering from a single point in the plasma which entails signal + background and background data sets. . . . .

Figure 20: Collective Thomson scattered signal obtained after background 


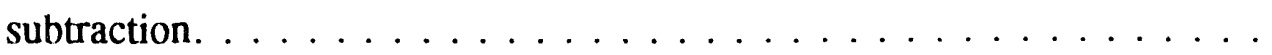

Figure 21: Time dependent electron density at $\mathrm{r}=0$ and $\mathrm{z}=65 \mu \mathrm{m}$ as computed by LASNEX. The 5ns (FWHM) Gaussian diagnostic pulse is included for reference. . . . . . . . . . . . . . . . .

Figure 22: Time dependent electron temperature at $\mathrm{r}=0$ and $\mathrm{z}=65 \mu \mathrm{m}$ as computed by LASNEX. The 5ns (FWHM) Gaussian diagnostic pulse is



Figure 23: Time dependent average ionization state at $r=0$ and $z=65 \mu \mathrm{m}$ as computed by LASNEX. The 5ns (FWHM) Gaussian diagnostic pulse is included for reference. $\ldots \ldots \ldots \ldots \ldots \ldots \ldots \ldots \ldots \ldots \ldots$

Figure 24: The parameter $B_{a d j}$ at $r=0$ as a function of the axial distance $z . \quad \ldots \quad 78$

Figure 25: Pulse height analyzer output of $266 \mathrm{~nm}$ diagnostic beam energy from the Rayleigh calibration. The RMS pulse-to-pulse amplitude fluctuation of the diagnostic beam energy is $\pm 4 \%, \ldots \ldots \ldots \ldots \ldots \ldots$

Figure 26: Calibration curve for the normalized counts $\left\langle\mathrm{E}_{\mathrm{sc}}{ }^{\mathrm{R}}>\right.$ from Rayleigh scattering in oxygen. The best fit (line) to the data (circles) has a slope of $\mathrm{m}=3.96 \times 10^{-4}$ Torr $^{-1} . \ldots \ldots \ldots \ldots \ldots \ldots$

Figure 27: Radial $\mathrm{x}-\mathrm{y}$ plane at an arbitrary $\mathrm{z}$ position in the plasma. Assuming a cylindrically symmetric plasma, an on-axis diagnostic beam will produce a symmetric Doppler shift in the Thomson signal about $x=0 . \ldots \ldots 85$


Thomson spectra collected at the same point in the plasma. The solid line 
$(-)$ is the average of the independent spectra. $\ldots \ldots \ldots \ldots \ldots$

Figure 29: Thomson spectra taken at the same point in the plasma for different diagnostic intensities. The sound speed, peak separation, is independent of diagnostic intensity. . . . . . . . . . . . . .

Figure 30: Contour plot of the experimentally measured normalized electron density $\left(n_{\mathrm{e}} / \mathrm{n}_{\mathrm{c}}\right)$ from collective Thomson scattering. . . . . . . 90

Figure 31: Contour plot of the experimentally measured $(\mathrm{Z}+5 / 3) \mathrm{T}_{\mathrm{e}}(\mathrm{eV})$ product frorn collective Thomson scattering. . . . . . . . . . . .

Figure 32: Contour plot of the experimentally measured normalized radial drift velocity $\left(\mathrm{U}_{\mathrm{rad}} / \mathrm{c}_{\mathrm{s}}\right)$ from collective Thomson scattering. The circles are the

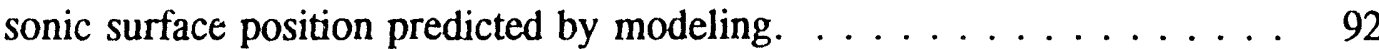

Figure 33: Approximate time $\tau_{\mathrm{e}}$ necessary to reach a given ionization state $\mathrm{Z}$ in a plasma with $\mathrm{T}_{\mathrm{e}}=40 \mathrm{eV}$ and a range of electron densities $\left(10^{19}-10^{20}\right.$



Figure 34: Electron densities required as a function of electron temperature for an aluminum species to be in LTE. An aluminum species is in LTE when the density lies above the line for that species.

Figure 35: The temperature dependent average ionization state for an aluminum plasma with $\mathrm{n}_{\mathrm{e}}=10^{19} \mathrm{~cm}^{-3}$ as predicted by the three models 1) LTE, 2) NLTE (ionization balance), and 3) coronal. . . . . . . . . . . . 95

Figure 36: Measured electron density $\left({ }^{*}\right)$ on axis as a function of axial distance. Results from a LASNEX simulation (-) and the analytical spherical 
model (- -) are included for comparison.

Figure 37: Measured sound speed $\left(^{*}\right)$ on axis as a function of axial distance. Results from a LASNEX simulation (-) and the analytical spherical model $(--)$ are included for comparison. . . . . . . . . . . . . 10

Figure 38: Measured electron density $\left({ }^{*}\right)$ at $\mathrm{z}=165 \mu \mathrm{m}$ as a function of radial distance. Results from a LASNEX simulation at $\mathrm{z}=150 \mu \mathrm{m}(-)$ and $\mathrm{z}=180 \mu \mathrm{m}(---)$ are included for comparison

102

Figure 39: Measured sound speed $(*)$ at $\mathrm{z}=165 \mu \mathrm{m}$ as a function of radial distance. Results from a LASNEX simulation at $\mathrm{z}=150 \mu \mathrm{m}$ (-) and $\mathrm{z}=180 \mu \mathrm{m}(---)$ are included for comparison.

Figure 40: Measured radial drift velocity $\left(^{*}\right)$ at $\mathrm{z}=165 \mu \mathrm{m}$ as a function of radial distance. Results from a LASNEX simulation (-) are included for comparison. ............................ 104

Figure 41: Refractive turning calculations for a $532 \mathrm{~nm}$ and $266 \mathrm{~nm}$ diagnostic beam as a function of axial distance in the laser plasma. The calculations are based on the LASNEX simulation results for the plasma. . . . . . 110

Figure 42: Raw collective Thomson scattering data showing inconsistent peak height asymmetries for different radial positions at an axial distance of

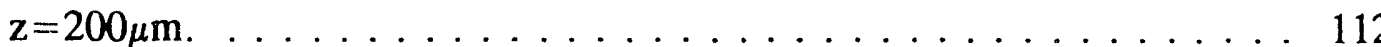

Figure 43: The ion-acoustic spectrum calculated from collisionless theory (- -) and from the modified fluid model (-) with $\mathrm{ZT}_{\mathrm{e}} / \mathrm{Ti}=8$ and $\left(\mathrm{k}_{\mathrm{ia}} \cdot \mathrm{U}_{\mathrm{d}}\right) / \mathrm{k}_{\mathrm{ia}} \mathrm{v}_{\mathrm{e}}$ $=02$ 
Figure 44: Reduction in the damping of the entropy wave from collisional 'Braginskii' theory when $\mathrm{k}_{\mathrm{ent}} \lambda_{\mathrm{ii}} \approx 1$ for a ratio of $\mathrm{ZT}_{\mathrm{e}} / \mathrm{T}_{\mathrm{i}}=4 . \ldots 137$

Figure 45: Reduction in the ion thermal conductivity $\kappa_{2}$ for the entropy wave from the collisional 'Braginskii' limit when $\mathrm{k}_{\mathrm{ent}} \lambda_{\mathrm{ii}} \approx 1$ for a ratio of

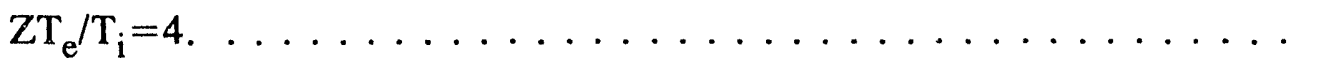

Figure 46: Damping of the ion-acoustic wave found from the solution of the Fokker-Planck equation for $\mathrm{ZT}_{\mathrm{e}} / \mathrm{T}_{\mathrm{i}}=8$. Collisional 'Braginskii' theory and the collisionless 'Landau' limit are included. . . . . . . . . . . .

Figure 47: Phase-amplitude normalized real part of the perturbed distribution function for the ion-acoustic wave with $\mathrm{ZT}_{\mathrm{e}} / \mathrm{T}_{\mathrm{i}}=8$ and $\mathrm{k}_{\mathrm{ia}} \lambda_{\mathrm{ii}}=0.133$ (dashes), 0.532 (dots), and 2.66 (line). . . . . . . . . . . . 142

Figure 48: Phase-amplitude normalized imaginary part of the perturbed distribution function for the ion-acoustic wave with $\mathrm{ZT}_{\mathrm{e}} / \mathrm{T}_{\mathrm{i}}=8$ and $\mathrm{k}_{\mathrm{ia}} \lambda_{\mathrm{ii}}=0.133$ (dashes), 0.532 (dots), 2.66 (line). . . . . . . . . .

Figure 49: The ion-acoustic damping calculated from the modified fluid model (circles) compared to the exact damping from the eigenvalue solution of the Fokker-Planck equation (line) for $\mathrm{ZT}_{\mathrm{e}} / \mathrm{T}_{\mathrm{i}}=8 \ldots \ldots \ldots \ldots$

Figure 50: Peak height asymmetry variation with the ion collisionality $\left(\mathrm{k}_{\mathrm{ia}} \lambda_{\mathrm{ii}}\right)$ for fixed $\mathrm{ZT}_{\mathrm{e}} / \mathrm{T}_{\mathrm{i}}=8$ and relative $\operatorname{drift}\left(\mathrm{k}_{\mathrm{ia}} \cdot \mathrm{U}_{\mathrm{d}}\right) / \mathrm{k}_{\mathrm{ia}} \mathrm{v}_{\mathrm{e}}=0.02 \ldots \ldots 146$

Figure 51: Modified fluid model fit (solid line) to Thomson spectra (dashed line) from a point in the plasma with axial position $=200 \mu \mathrm{m}$, radial position $=-25 \mu \mathrm{m}$. 
Figure 52: Optical schematic of the collection system which couples the Thomson scattered signal from the plasma onto the CCD detector for the peak height asymmetry analysis. $\ldots \ldots \ldots \ldots \ldots \ldots \ldots \ldots \ldots \ldots$

Figure 53: Optical schematic of the spectrometer used for the Thomson scattering experiments. A cylindrical lens is added to provide astigmatic

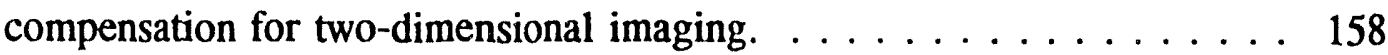

Figure 54: $\mathrm{CCD} /$ Spectrometer imaging of $100 \mu \mathrm{m}$ wire mesh with $150 \mu \mathrm{m}$ spacing. The horizontal wires are not visible due to the astigmatism of the

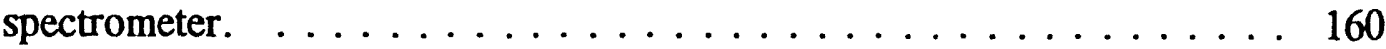

Figure 55: CCD/Compensated Spectrometer imaging of $100 \mu \mathrm{m}$ wire mesh with




Table I: Conductivity coefficients $\left(\kappa_{1}, \kappa_{2}\right)$ in the quasineutral Landau limit.

Also, the ion-acoustic frequency from Landau theory and the modified fluid model. ........................... 125

Table II: The damping coefficients ${\gamma_{\mathrm{ia}}}^{\circ}$ and $\gamma_{\mathrm{ent}}{ }^{\circ}$ for the ion-acoustic and entropy waves in the collisional limit. . . . . . . . . . . . . . . . . 134

Table III: Damping and ion thermal conductivity for the entropy wave as a function of $\mathrm{k}_{\text {ent }} \lambda_{\mathrm{ii}}$ for $\mathrm{ZT}_{\mathrm{e}} / \mathrm{T}_{\mathrm{i}}$ ratios of 2,4 , and $8 \ldots \ldots 136$

Table IV: Eigenvalue results for the complex ion-acoustic frequency with $\mathrm{ZT}_{\mathrm{e}} / \mathrm{T}_{\mathrm{i}}=8$. (ADI) results from Ref. 151 are included for comparison. . . 140 Table V: Collisionally dependent transport coefficients and ratio of specific heats for the ion-acoustic wave with $\mathrm{ZT}_{\mathrm{e}} / \mathrm{T}_{\mathrm{i}}=8 \ldots \ldots \ldots 144$ 
Chapter I: Laser-produced plasma diagnostics

Only one plasma diagnostic is needed to completely diagnose the parameters of a given plasma. This plasma diagnostic provides one with the distribution function for all species of the particular plasma at every point in that plasma for the entire evolution of the plasma. With this distribution function, $\mathscr{F}(\mathbf{x}, \mathbf{v}, \mathbf{t})=\Sigma \mathrm{f}_{\text {species }}(\mathbf{x}, \mathbf{v}, \mathrm{t})$, any macroscopic quantity as a function of position and time can be determined by calculating a weighted average of the corresponding microscopic quantity over velocity space with the distribution function. For example, the microscopic quantity $1 / 2 \mathrm{mv}^{2}$ is the kinetic energy of a particle. The macroscopic counterpart, temperature $\theta(x, t)$, can be calculated by performing the integral ${ }^{3} / 2 \theta(x, t)=\int 1 /{ }_{2} \mathrm{mv}^{2} \mathscr{F}(\mathrm{x}, \mathrm{v}, \mathrm{t}) \mathrm{d}^{3} \mathrm{v}$. Unfortunately, this perfect plasma diagnostic does not exist, and it probably never will. Therefore, a plasma physicist is left with choosing a diagnostic or multiple diagnostics each of which provide a small piece of vital information about the plasma, and hopefully this information is enough to investigate the problem of interest.

Plasma diagnostics fall under one of three categories: self-emissive, refractive/absorptive, and scattering. The self-emissive diagnostics operate with a detector, only, and the detector monitors some quantity which is supplied by the plasma. Diagnostics which fall under the other two categories require both a detector and a probe. The detector monitors a change in the probe due to an interaction with the plasma. There are many diagnostics which fall under these categories; to cover all of them would be nearly impossible and beyond the scope of this dissertation. Therefore, a small sampling 
of diagnostics will be presented that are useful in measuring characteristics of laserproduced plasmas.

Laser-produced plasmas possess certain characteristics which limit the type of diagnostics that can be used on them. These plasmas are small, typically less than one millimeter in size. Therefore, a diagnostic must have submillimeter resolution to measure the spatial scale lengths present in these plasmas. Pulsed lasers are normally used for plasma production. The laser pulse durations can be as long as one hundred nanoseconds (ns), but due to recent developments in laser technology, can be as short as one hundred femtoseconds $(\mathrm{fs})^{1,2}$. The plasmas formed by these lasers evolve very quickly, and if temporal information is required, a diagnostic must be able to provide resolution on these time scales. Laser-produced plasmas have a wide variation of electron densities ranging from $10^{17}-10^{21} \mathrm{~cm}^{-3}$ for gas targets to $10^{19}-10^{23} \mathrm{~cm}^{-3}$ for solid targets to $10^{26} \mathrm{~cm}^{-3}$ for Inertial Confinement Fusion (ICF) pellet targets. The temperatures of these plasmas cover a broad range as well from only a few electron volts (eV) to several thousand electron volts (keV). Therefore, laser-plasma diagnostics must be able to operate over a large range of densities and temperatures.

Laser-produced plasma diagnostics must take into account the internally generated electric (E) and magnetic fields (B) of laser-produced plasmas. One process by which an internal electric field can be generated is easily demonstrated by considering the fundamental criteria for plasmas, quasineutrality. The necessary condition imposed by quasineutrality on any electric current density (j) that may be present in the plasma is, 


$$
\vec{\nabla} \cdot \vec{j}=0
$$

In other words, there can be no net charge flow into or out of a plasma. The electric current density can be related to the electric field in a plasma through the generalized Ohm's law ${ }^{3}$,

$$
\vec{j}=\sigma \cdot\left(\vec{E}+\frac{\vec{v}}{c} x \vec{B}+\frac{k T_{e}}{e n_{e}} \vec{\nabla} n_{e}-\frac{\vec{j} \times \vec{B}}{c e n_{e}}\right)+\vec{\tau} \cdot \vec{\nabla} T_{e}
$$

where $\sigma$ is the conductivity, $\mathbf{v}$ is the mass velocity, $\boldsymbol{\tau}$ is the thermoelectric coefficient at constant density, and $n_{e}, T_{e}$, and $e$ are the electron density, electron temperature, and electron charge respectively. A sufficient condition for $\mathrm{Eq}$. (1) is $(\mathrm{j}=0)$, and when substituted into Eq. (2), neglecting magnetic field effects, an equation for the electric field in terms of gradients in temperature and density is obtained. Therefore when the sufficient condition $(j=0)$ for quasineutrality is satisfied, electric fields are generated via gradients in temperature and density. Spontaneous magnetic fields can also exist in laserproduced plasmas ${ }^{4,5,6}$. The source for magnetic field generation is derived by taking Eq. (2), solving for the electric field (E), and substituting this expression into Maxwell's equation $\partial \mathrm{B} / \partial \mathrm{t}=-\mathrm{c} \nabla \times \mathbf{E}$. The resulting equation for the time dependent magnetic field is (Ref. 4),

$$
\frac{\partial \vec{B}}{\partial t}=\vec{\nabla} \times(\vec{v} \times \vec{B})+\frac{c^{2}}{4 \pi \sigma} \vec{\nabla} \vec{B}-\left[c \vec{\nabla} x\left(\vec{\tau} \cdot \vec{\nabla} r_{e}\right)+\frac{c k}{e n_{e}} \vec{\nabla} n_{e} x \vec{\nabla} T_{e}\right]
$$

where the $(j \times$ B) Hall term has been neglected. The final two terms bracketed in Eq. (3) 
are the source terms for the magnetic field. If the thermoelectric coefficient is a scalar and independent of position, then the first term is zero, and a magnetic field is generated only by non-parallel gradients in electron temperature and electron density. Electric field effects are fairly easy to include in plasma diagnostic analysis, but considerable complexity is added when magnetic fields are included. Therefore, the effect of magnetic fields on laser-produced plasma diagnostics should be eliminated when possible. Magnetic fields could be eliminated entirely by producing plasmas in which the source terms in Eq. (3) are identically zero, but this method is not entirely practical. Another method involves making the magnetic field effects small by properly choosing the conditions under which a diagnostic is performed. The relative effect of magnetic fields on plasma diagnostics can be quantified by the ratio of the cyclotron frequency $(\Omega)$ to the characteristic frequency of the process being measured ( $\left.\omega_{\text {process }}\right)$. The electron cyclotron i:equency $\left(\Omega_{e}=e B / m_{e} c\right)$ is used if the process involves electron motion, and the ion cyclotron frequency $\left(\Omega_{\mathrm{i}}=\mathrm{ZeB} / \mathrm{m}_{\mathrm{i}} \mathrm{c}\right)$ is used if the process involves ion motion. To the extent that diagnostic conditions can be chosen to make this ratio small, magnetic field effects can be ignored.

One example of reducing magnetic field effects in laser-plasma measurements can be demonstrated through the diagnostic technique of interferometry, which will be discussed in more detail later in the refractive/absorptive diagnostic section. Interferometric diagnostics measure directly the spatially dependent refractive index of a plasma which for a fully ionized, magnetic plasma is given by the Appleton-Hartree formula to $b e^{7}$, 


$$
n^{2}(\omega)=1-\frac{\frac{\omega_{p}^{2}}{\omega^{2}}\left(1-\frac{\omega_{p}^{2}}{\omega^{2}}\right)}{1-\frac{\omega_{p}^{2}}{\omega^{2}}-\frac{1}{2} \frac{\Omega_{e}^{2}}{\omega^{2}} \sin ^{2} \theta \pm\left[\left(\frac{1}{2} \frac{\Omega_{e}^{2}}{\omega^{2}} \sin ^{2} \theta\right)^{2}+\left(1-\frac{\omega_{p}^{2}}{\omega^{2}}\right)^{2} \frac{\Omega_{e}^{2}}{\omega^{2}} \cos ^{2} \theta\right]^{\frac{1}{2}}}
$$

where $\theta$ is the angle between the direction of electromagnetic propagation and the magnetic field, and $\omega_{\mathrm{p}}$ is the electron plasma frequency given by ${ }^{8,9}$,

$$
\omega_{p}=\sqrt{\left(\frac{4 \pi e^{2} n_{e}}{m_{e}}\right)}=5.64 \times 10^{4} n_{e}^{1 / 2}
$$

Therefore the refractive index is a direct function of the electron density $n_{e}\left(\mathrm{~cm}^{-3}\right)$ and unfortunately the magnetic field, a quantity which is typically unknown. Since interferometry measures the refractive index, the electron density can also be measured with an accuracy that is partially dependent on eliminating the magnetic field dependence in Eq. (4). This is accomplished by choosing a diagnostic frequency ( $\omega)$ large enough such that the ratio $\left(\Omega_{\mathrm{e}} / \omega\right)$ is small. Eliminating the complicating effect of the magnetic field does allow for a more accurate measurement of the electron density in this case, but does by no means indicate the magnetic field is not influencing the plasma quantities being measured. It is a well known fact that magnetic fields effect the transport coefficients of a plasma ${ }^{10,11}$, and can therefore impede heat flow ${ }^{12,13}$. These types of effects can make the characteristics of a magnetic plasma different from a plasma with no magnetic field. 
The refractive index of a fully-ionized, non-magnetic plasma is obtained by setting $\Omega_{\mathrm{e}}=0$ in Eq. (4),

$$
n(\omega)=\sqrt{\left(1-\frac{\omega_{p}^{2}}{\omega^{2}}\right)}
$$

and is always less than or equal to 1.0 for $\omega \leq \omega_{\mathrm{p}}$. This optical characteristic is opposite from all other forms of matter: gases, liquids, and solids, which exhibit an index of refraction greater or equal to 1.0. Since the refractive index is a function of electron plasma density and laser-produced plasmas typically have large density gradients, the refractive index will also have large gradients and the plasma will appear as a short focal length lens to electromagnetic waves. Therefore, electromagnetic waves will not travel in a straight line through the plasma but their paths will bend by the process of refractive turning. In the limiting case when the frequency $(\omega)$ in question approaches the plasma frequency $\left(\omega_{\mathrm{p}}\right)$, the wave will become evanescent and reflection will occur. The density at which the wave becomes evanescent is defined as the critical density $\left(n_{c}\right)$, and can be calculated as a function of $(\omega)$ by setting the index of refraction in Eq. (6) equal to zero and using the definition in Eq. (5) for the plasma frequency.

$$
n_{c}=\left(\frac{m_{e}}{4 \pi e^{2}}\right) \omega^{2}=3.15 \times 10^{-10} \omega^{2} \mathrm{~cm}^{-3}
$$

Given a radiation frequency, the critical density defines an absolute limit to how far radiation can propagate into or out of a plasma, and refractive turning can limit the 
accessible region even further ${ }^{14}$. The combination of refractive turning and electron densities above critical impose limitations on all three categories of laser-plasma diagnostics.

Electromagnetic absorption and radiation are two other optical properties of plasmas that play a role in laser-plasma diagnostics. The absorption and radiation characteristics of laser-produced plasmas are governed predominately by the atomic processes of bound-bound, free-bound, and free-free transitions ${ }^{15,16}$ and to a lesser extent by cyclotron and Cerenkov radiation (Ref. 7). Bound-bound transitions occur when an electron in a bound state of an atom or an ion makes a transition to another bound state. Line emission and resonant line absorption are the radiation and absorption mechanisms respectively of bound-bound transitions. Radiative free-bound transitions occur when an electron in a continuum state is captured by an ion by recombination. The inverse absorptive transition is photoionization. Bound-bound and free-bound transitions do not always need to involve the absorption or emission of a photon. For example, collisional excitation and de-excitation can cause bound-bound transitions to occur without involving a photon, and three-body recombination, collisional ionization, dielectronic recombination, and autoionization are non-photon associated free-bound transitions. Finally, free-free transitions occur when an electron in a continuum state collides with an ion and makes a transition to another continuum state. When the electron looses energy, bremsstrahlung radiation is emitted, and when the electror gains energy, inverse bremsstrahlung absorption occurs. Bound-bound transitions exhibit sharp features in their absorption and radiation spectra, on the other hand free-bound and free-free absorption 
and radiation are characterized by broad spectral (continuum) structure. Cyclotron radiation is due the acceleration of an electron in a magnetic field. If the electron-ion collision frequency $\left(v_{\mathrm{ei}}\right)$ is much less than the electron cyclotron freciuency and the magnetic field is spatially uniform, the radiation spectrum will appear as a series of harmonic peaks broadened by Doppler and relativistic effects. In laser-produced plasmas the opposite conditions typically occur. The electron-ion collision frequency is comparable to or greater than the electron cyclotron frequency and the magnetic fields will have large spatial variations due the small size of laser-plasmas. These two conditions independently will randomize the motion of the electron, thereby destroying the cyclotron resonance and eliminating the harmonic peaks from the emission spectrum of the plasma. Cerenkov emission can be generated by either the relativistic motion of electrons in a magnetized plasma (Ref. 7) or through the process of inverse Landau damping where no magnetic field is necessary (Ref. 16).

These radiative and absorptive processes can be a mixed blessing for laser-plasma diagnostics. For example, in a plasma with a Maxwellian distribution of velocities, the bremsstrahlung emissivity $\epsilon_{b}(\omega)$ is unpolarized, spatially isotropic, and is given by (Ref. 15),

$$
\epsilon_{b}(\omega)=8.67 \times 10^{-41} \frac{Z n_{e}^{2}}{T_{e}^{1 / 2}} e^{-\left(\frac{\hbar \omega}{k T_{e}}\right)} G_{f f}
$$

where the emissivity is in units of watts per cubic centimeter of plasma per steradian per 
unit frequency interval, $\mathrm{n}_{\mathrm{e}}\left(\mathrm{cm}^{-3}\right)$ and $\mathrm{T}_{\mathrm{e}}\left({ }^{\mathrm{O}} \mathrm{K}\right)$ are the electron density and temperature, $\mathrm{Z}$ is the ionization state, and $\mathrm{G}_{\mathrm{ff}}$ is the Gaunt factor; it is a dimensionless quantity ranging from 1 to 10 . The log of the bremsstrahlung emissivity has a slope of $1 / \mathrm{kT}_{\mathrm{e}}$ as a function of $\omega$, and this relationship can be used by spectroscopic diagnostics to accurately measure the electron temperature of a plasma. Just as the bremsstrahlung emission helps in the diagnosis of the plasma, inverse bremsstrahlung absorption can hinder spectroscopic diagnostics by making the plasma opaque over some ranges of electromagnetic frequencies. The inverse bremsstrahlung absorption length for electromagnetic frequencies $(\omega)$ much greater than the electron-ion collision frequency $\left(v_{\mathrm{ei}}\right)$ is given by (Ref. 14),

$$
\alpha_{b}=\frac{\omega_{p}^{2} v_{c i}}{\omega^{2} c}\left(1-\frac{\omega_{p}^{2}}{\omega^{2}}\right)^{\frac{-1}{2}}
$$

This absorption mechanism can also be a hinderance to laser diagnostics. A diagnostic laser beam can not only lose energy passing through the plasma, but if the energy loss is large enough, local plasma heating can occur thus making the diagnostic undesirably intrusive.

The first group of laser-produced plasma diagnostics that will be discussed fall under the category of self-emissive diagnostics. As mentioned earlier, these diagnostics require a only a detector which monitors some quantity supplied by the plasma. Most diagnostics in this category are electromagnetic related, but there is a small group of diagnostics that detect particle emission from laser-produced plasmas.

The energy and angular distribution of electrons emitted from a laser-produced 
plasma can be measured with magnetic spectrometers ${ }^{17}$. The energy distribution of electrons yields information about the high-energy tails that exist in the electron distribution function of plasmas generated by high-intensity laser pulses. The suprathermal electrons in the high-energy tail have very long mean-free paths and 'decouple' (Ref. 16) from the rest of the plasma. This effect robs energy from the laser generating the plasma that could otherwise be used for ablation and compression ${ }^{18}$. The hot electrons can also cause preheat in the fuel of ICF targets ${ }^{19,20,21}$. The amount of laser radiation expended in generating the fast electrons can be obtained by integrating over the energy and angular measurements (Ref. 17). Ion emission from laser-produced plasmas can also be measured with charge or Faraday $\operatorname{cups}^{22}$ or by Thomson ion spectrometers ${ }^{23}$. A strong correlation has been shown to exist between the mean fast ion energy and the hot electron temperatures of several laser-matter interaction experiments ${ }^{24}$ : Therefore, ion diagnostics might be used indirectly for hot electron diagnostics. Neutron diagnostics are another form of particle diagnostics which prove useful for ICF plasmas, where the neutron yields can be measured by $\mathrm{Ag}$ activation detectors and scintillators ${ }^{25}$ or currentmode time-of-flight detectors ${ }^{26}$. Measurements of the neutron energy spectrum can provide a value for the thermal ion fuel temperatures (Refs. 7, 25, 26).

All of the remaining self-emissive diagnostics can be placed under the subtitle, plasma spectroscopy. Plasma spectroscopy has been used for a long time as an effective diagnostic for laser-produced plasmas (Ref. 15). Spectroscopic techniques can be used to measure many of the quantities characterizing laser-produced plasmas: electron densities, electron and ion temperatures, magnetic and electric fields, particle velocities, velocity 
distributions, energy transport, mass ablation rate, and plasma evolution. The electron density is obtained typically by measuring the Stark broadening of optical lines emitted from bound-bound transitions. Lines in the $\mathrm{x}$-ray region of the spectrum ${ }^{27,28}$ are normally used, but Stark broadened lines from the visible/ultraviolet region work as well $^{29}$. The electron density can also be calculated from absolute line intensities or ratios of optical lines which depend on electron density and not electron temperature $e^{30}$. The ion temperature might be diagnosed through the Doppler broadening of optical lines (Ref. 7). Doppler broadening is caused by the thermal motion of the ions, therefore the magnitude of the effect is a direct measure of the ion temperature. Although both Stark and Doppler broadening effects can be present in comparable amounts simultaneously, thus making line width analysis difficult, the effects are easily decoupled by judicious choice of diagnostic location in the plasma. Doppler broadening will dominate in regions of the plasma which possess large ion temperatures and low electron densities, and Stark broadening will be largest in regions of high electron density and moderate ion temperature. The electron temperature of a laser-produced plasma can be measured in one of three ways: the slope of the frequency spectrum of bremsstrahlung emission ${ }^{31}$ as outlined by Eq. (8), relative line to continuum measurements (Ref. 15), and optical line intensity ratios (Refs. 28, 29, 30, 32, 33). Magnetic fields can cause line emission profiles to be polarized through the Zeeman effect. The magnetic field strengths are measured from the polarization dependence of these line profiles ${ }^{34}$. The spontaneous magnetic fields a laser-produced plasma have also been diagnosed with a magnetic field probe in proximity of the plasma (Ref. 31 ), but due to the small size of laser-produced 
plasmas this diagnostic technique has limited application. Electric fields can also cause polarization effects in line emission. An electric field, unlike magnetic fields, causes the line intensity to be polarization dependent, and can be measured through this effect ${ }^{35}$. The measurement of electron densities, electron and ion temperatures, magnetic and electric fields through the spectroscopic diagnostics outlined here require detailed theoretical models. These models are dependent on the underlying conditions of the plasma. Whether the plasma is in local thermal equilibrium (LTE), coronal equilibrium, coliision-radiative equilibrium (Ref. 16), or some other characteristic state definitely effects the theoretical modeling necessary for analyzing data and the accuracy of the results.

There exists a group of spectroscopic diagnostics which require much less theoretical modeling for quantitative measurements of laser-produced plasmas. Doppler effects from bulk ion motion will shift emission lines from their normal spectral positions. The magnitude of this shift can yield information about the ion particle velocities in the laser-plasma (Refs. 7, 32). Suprathermal electrons present in the electron velocity distribution of a laser-produced plasma are detected through bremsstrahlung emission. The interaction of suprathermal electrons with background plasma ions produces a distinctive suprathermal component in the bremsstrahlung radiation spectrum which can be detected experimentally $36,37,38,39$. By layering targets with various materials of different $x$-ray spectral emission characteristics, the energy transport and mass ablation rate in a laser-produced plasma can be diagnosed by monitoring the spatial and time dependent evolution of these layers through their characteristic $\mathrm{x}$-ray spectra 
(Ref. 21). Plasma evolution characteristics may be monitored by two-dimensional imaging of selective regions of the $x$-ray emission spectrum of a laser-produced plasma. An $x$-ray region is chosen by placing the appropriate $x$-ray spectral filter in the diagnostic line of sight, and then using an imaging apparatus to record the spatially dependent, filtered, $\mathrm{x}$ ray emission. A common imaging technique involves the use of pinhole camera. Pinhole cameras are attractive because they are cheap, simple, and provide fairly good spatial resolutions. The resolution is on the order of the pinhole size, ignoring diffractive effects, and can be as fine as 5-10 microns. Pinhole cameras have been used to look at the size of $x$-ray emitting regions in laser-produced plasmas ${ }^{40}$. When pinhole cameras are used in conjunction with microchannel plates (MCP) in a stripline configuration, gated, time sequenced, snap shots of imploding (ICF) targets can be taken $41,42,43,44$. This represents a very powerful technique for monitoring the two-dimensional evolution of a laser-produced plasma. X-ray microscopes ${ }^{45}$ and $\mathrm{X}$-ray shadowgraphy (Ref. 40) are variations on the pinhole camera that have been used to provide better spatial resolution under certain circumstances. The use of an annular (ring) aperture instead of a pinhole is another variation that has been shown to improve the signal-to-noise in images as much as a factor of ten. Three dimensional measurements can actually be made by utilizing the data from several pinhole cameras along different lines of sight with computed tomography. This diagnostic has produced three-dimensional images of electron density and temperature in a laser-produced plasma ${ }^{46}$.

The second group of laser-produced plasma diagnostics are filed under the category of refractive/absorptive diagnostics. Refractive/absorptive diagnostics unlike the 
self-emissive diagnostics require both a detector and a probe to operate. Refractive diagnostics probe the refractive index variations in a laser-produced plasma, and there are several geometries employed for performing these measurements: interferometry, holographic interferometry, schlieren imaging, and shadowgraph imaging. Interferometry and holographic interferometry require two optical beams, a reference beam and a probe beam, which are both derived from the same source (Ref. 7). The probe beam develops spatially dependent, wave front phase shifts when it passes through a plasma, and when recombined with the reference beam produces an interferometric image of the plasma. This image contains a series of fringes (isophase contours), and by measuring fringe shifts in the image, the spatially dependent phase of the probe is obtained. Since the phase shifts in the probe are due to refractive index variations in the plasma, the electron density can be measured from these diagnostics $47,48,49$. Interferometry has successfully been used to study filamentation in laser-produced plasmas ${ }^{50}$, and holographic interferometry has also been utilized to look at radiation-pressure effects in laser-produced plasmas ${ }^{51}$. Interferometry differs from holographic interferometry in that interferometry produces a real image that can immediately be recorded on film. Holographic interferometry requires a read out process after the fact to produce a real image. Holographic interferometry has the advantage of allowing several different images to be recorded on the same piece of film, from which each image can be read out individually at a later time $e^{52}$. Schlieren and shadowgraph imaging require only one diagnostic beam to operate, and are sensitive to the first and second spatial derivatives of the refractive index respectively (Ref. 7 ). Schlieren imaging is performed by passing a parallel probe beam through the plasma and 
collecting only those rays that are refractively turned through a given range of angles. The image produced from these rays is in the form of contours of deflection angle in the plasma. The deflection contours are usually shaped like the electron density contours in the plasma and are used for monitoring plasma expansion characteristics (Ref. 32). Shadowgraph imaging involves sending a beam with a uniformly intense cross-section through a plasma and imaging the modified beam profile on the other side. The beam profile is modified by refractive turning in the plasma, and these effects appear as intensity variations across the once uniform beam. Shadowgraph imaging diagnostics have been used for monitoring whole beam self-focusing and filamentation on laser beams passing through laser-produced plasmas $^{53,54,55,56}$. All of the refractive diagnostics share a common drawback, the refractive effects on the diagnostic beam are line integrated as the beam passes through the plasma. Therefore, to deduce the properties of the plasma at a given point along the diagnostic beam path requires some theoretical modeling. However, when the plasma is cylindrically symmetric the problem is simply reduced to performing an Abel inversion on the data (Ref. 7).

Absorptive diagnostics are used for measuring a variety of laser-produced plasma quantities. Laser-produced plasmas can absorb light by many different processes. Two of these absorption processes were outlined earlier: resonant line absorption due to boundbound transitions, and inverse bremsstrahlung (Eq. (9)). The resonant line absorption due to bound-bound transitions has been used with $\mathrm{x}$-ray backlighting diagnostics to measure density, temperature and ionization profiles in laser-produced plasmas ${ }^{57,58,59}$. Xray backlighting is a technique which involves generating a separate laser-produced 
plasma as a source of $x$-rays. This source is placed behind the plasma to be diagnosed and the absorptive characteristics of the plasma on the source emission are measured spectroscopically. The inverse bremsstrahlung absorption process has also been used with $\mathrm{x}$-ray backlighting to measure the Rayleigh-Taylor instability $60,61,62,63,64$ and nonuniformity imprints on laser-irradiated target surfaces ${ }^{65}$. The resonant line absorption due to bound-bound transitions should not be confused with resonance absorption which is a third important absorption mechanism in laser-produced plasmas. Resonance absorption occurs when an optical wave passes through a plasma near its critical density (Eq. (7)), and its electric field has a component pointing along the electron density gradient (Ref. 14). Since the electron plasma frequency $\left(\omega_{p}\right)$ is equal to the light frequency $(\omega)$ at the critical surface, electron plasma waves can be resonantly excited at this point thus robbing energy from the optical wave. This resonance absorption process has been used as a diagnostic for measuring density scale lengths in laserproduced plasmas $^{66}$.

Scattering diagnostics is the third category that laser-produced plasma diagnostics can fall under. Light scattering of an optical beam in a plasma occurs when a charged particle in the plasma is accelerated in the electromagnetic field of the beam. The accelerating particle emits radiation in all directions and the emitted radiation is the scattered wave (Ref. 7). The scattering is due primarily to electrons which are accelerated more than ions in the field due to their small mass. The scattered radiation can be coherent or incoherent depending on whether the electrons that are scattering the optical beam exhibit correlated or uncorrelated motion. The scattering category is subdivided into 
two groups: self-scattering diagnostics and probe scattering diagnostics.

Self-scattering diagnostics use the same beam that forms the plasma as a probe beam for the diagnostic. These diagnostics detect light scattering from high intensity laser beams that generate parametric instabilities in the plasma they are forming. Common laser-produced plasma instabilities include: stimulated Raman scattering (SRS), stimulated Compton scattering (SCS), stimulated Brillouin scattering (SBS), self-focusing or filamentation, ion acoustic parametric decay instability (IADI), and the two plasmon decay instability. Describing each instability in detail would require a great deal of length so the reader is directed to the literature on this subject matter ${ }^{67,68,69,70}$. In brief, self-scattering diagnostics are used to monitor instabilities generated in laser-produced plasmas in attempt to understand the scaling laws that govern the instabilities and the basic plasma physics issues that surround them. For example, the collisional and Landau damping effects on SRS spectra has been studied ${ }^{71,72}$ as well as stimulated Raman scattering in long-scale-length plasmas ${ }^{73,74}$. Experiments on SCS have been performed in laser-produced plasmas to measure the intensity dependence of the instability ${ }^{75}$. By observing the temporal behavior of SBS backscattering in long-scalelength plasmas, information about which plasma conditions are most likely to drive SBS is obtained ${ }^{76}$ and energy loss due to SBS in a laser-produced plasma has been monitored by integrating SBS sidescattering ${ }^{77}$. Experiments have been performed that show filamentation due to the self-focusing of a laser beam in a plasma can be monitored by second harmonic scattering ${ }^{78}$. Second harmonic spectrum can also result from $\mathrm{IADI}^{79}$ and experiments have been conducted to determine the threshold and intensity 
dependence of the instability on the ionization state of the plasma ${ }^{80}$. The TPD instability can be detected through $3 / 2$ harmonic scattering and the influence of laser beam smoothing techniques on this instability have been analyzed ${ }^{81}$. Self-scattering diagnostics are not only used for instability analysis; they can also be used for measuring the electron temperature and peak electron density of a laser-produced plasma. For example, the Landau cutoff in SRS backscattering has been used for an electron temperature diagnostic ${ }^{82}$ and spectrally narrow SRS emission has been used for peak electron density measurements (Ref. 32).

The probe scattering diagnostics for laser-produced plasmas can be either Thomson scattering or Compton scattering. Thomson scattering occurs when the incident photon energy $(h v)$ is much less than the rest energy of the electron $\left(m_{e} c^{2}\right)$. Compton scattering results in the opposite limit where the incident photon energy is much greater than the rest energy of the electron $\left(h v * m_{e} c^{2}\right)$. Probe wavelengths for plasma diagnostics are typically in the visible-ultraviolet range of the spectrum in which photon energies are less than six electron volts $(6 \mathrm{eV})$. Since the rest energy of an electron is $511 \mathrm{keV}$, the Compton effect will be negligible for $6 \mathrm{eV}$ photons and only Thomson scattering diagnostics need be considered at this point. The very first Thomson scattering experiments on plasmas where conducted by radar scattering off of the ionosphere ${ }^{83,84}$. Thomson scattering utilizing lasers as probes soon followed on electron beams ${ }^{85}$ and theta-pinch plasmas ${ }^{86,87,88,89}$. More recently, Thomson scattering, coupled with theory, has been used to measure the electron and ion temperatures, electron and ion drift velocities, and the ionization state of a laser-produce 
plasma $^{90}$. The characteristics of a high density, cold, laser-induced, metal-vapor plasma have also been studied using Thomson scattering ${ }^{91}$. Thomson scattering has been used successfully to diagnose some of the parametric instabilities mentioned in the last paragraph that are associated with laser-plasma interactions: stimulated Raman scattering $92,93,94,95,96$, stimulated Brillouin scattering $97,98,99,100,101$, and the twoplasmon decay instability ${ }^{102,103,104}$. Plasma wave mode coupling between SRS and SBS generated waves has been investigated with Thomson scattering ${ }^{105}$, and the diagnostic has also been utilized to monitor the beat wave excitation of electron plasma waves $^{106,107}$. Although widely used as a laser-produced plasma diagnostic, Thomson scattering does have some disadvantages. In particular, Thomson scattering efficiencies are quite small especially when scattering occurs from thermal level motion in a laser-produced plasma. Thermal level scattering efficiencies can be as small as $10^{-11}$ of the incident probe energy and these scattered light levels make detection of the Thomson scattered signals difficult.

This chapter has presented some of the basic properties of a laser-produced plasma that must be considered when choosing a diagnostic. The laser-produced plasma diagnostics available to a research scientist have been covered in some detail as well. All laser-produced plasmas are not created equal and therefore there is no universal diagnostic for investigating them. The two most important considerations involved in choosing a diagnostic for a particular laser-produced plasma are 1) the limitations imposed by the properties of the given plasma on the diagnostic and 2) what plasma parameters and/or phenomena are trying to be measured. The plasma diagnostic should be ncnintrusive, i.e. 
not effect the quantities that are being measured, and if at all possible, a plasma diagnostic should require a minimum amount of modeling for interpreting data and be simple to operate.

Based on these considerations, collective Thomson scattering from thermal level ion-acoustic waves is chosen as the diagnostic for the collisional laser-produced plasma in this dissertation. The collisional laser-produced plasma being studied is generated from a low-energy ( $E_{L} \leq 1$ joule) long pulse (full width at half-maximum, FWHM $=9 \mathrm{~ns}$ ) laser (wavelength, $\lambda=1.06 \mu \mathrm{m}$ or critical density, $\mathrm{n}_{\mathrm{c}}=1 \times 10^{21} \mathrm{~cm}^{-3}$ ) focussed to a spot size $(F W H M=100 \pm 10 \mu \mathrm{m})$ with peak intensity of $2 \times 10^{11} \mathrm{~W} / \mathrm{cm}^{2}$ onto a rotating cylindrical target of aluminum. The high repetition rate $(\approx 10 \mathrm{~Hz})$ and high stability $(\approx 2 \%)$ of the laser provides both repetitive and reproducible plasma production, and with the relatively low peak intensity used, there are no instabilities present in the plasma that might hinder data analysis. Time integrated collective Thomson scattering provides a way to thoroughly diagnose the corona of the plasma near the peak of the laser pulse. The plasma is approximately stationary in the time during the diagnostic pulse $(\mathrm{FWHM} \approx 5 \mathrm{~ns})$ so that time resolution is not required. The axial and radial profiles of the electron density, sound speed, and the radial profile of the fluid velocity are measured. Ultraviolet diagnostic light at $266 \mathrm{~nm}$ is used to minimize the complicating effects mentioned earlier of inverse bremsstrahlung and refractive turning in the region of interest where electron densities $\approx \mathrm{n}_{\mathrm{c}} / 10$. Noise reduction techniques are used to generate high-quality Thomson scattering data with very good signal-to-noise ratios and the spatial profile of the corona (scale length $L_{s} \geq 100 \mu \mathrm{m}$ ) is well resolved by this 
diagnostic (axial resolution, $\Delta \mathrm{z} \approx 30 \mu \mathrm{m}$ and radial resolution, $\Delta \mathrm{r} \approx 30 \mu \mathrm{m}$ ). The data obtained from these scattering experiments is used to test the computational accuracy of two-dimensional LASNEX ${ }^{108}$ simulations, and the agreement between the experiment and LASNEX is good. The experimental results and LASNEX simulations agree within a percent standard deviation of $40 \%$ for the electron density and $50 \%$ for the sound speed and radial drift velocity. The data is also used to compare with a simple analytical model for a laser-heated plasma.

Collective Thomson scattering from ion-acoustic waves can not only be used to make accurate spatially resolved measurements of electron density, sound speed, and drift velocity, the diagnostic can also be used to obtain information about spatially dependent relative thermal flow velocities between ions and electrons. This measurement requires some new theory to be developed for Thomson scattering which is presented in this dissertation. The theory is based on the Fokker-Planck equation and provides an analytic model for predicting the lineshape of the ion-acoustic Thomson spectra from the plasma being studied which is characterized by $\left(0 \leq \mathrm{k}_{\mathrm{ia}} \lambda_{\mathrm{ii}} \leq \infty\right)$ and $\mathrm{ZT}_{\mathrm{e}} / \mathrm{T}_{\mathrm{i}}$, where $\mathrm{k}_{\mathrm{ia}}$ is the ion-acoustic wave number, $\lambda_{\mathrm{ii}}$ is the ion-ion mean free path, $\mathrm{Z}$ is the ionization state of the plasma, and $T_{e}, T_{i}$ are the electron and ion temperatures (eV), respectively. The electrons are assumed to be approxitnately collisionless on the scale of a wavelength of the ion-acoustic wave $\left(\mathrm{k}_{\mathrm{ia}} \lambda_{\mathrm{ei}}, \mathrm{k}_{\mathrm{ia}} \lambda_{e e} \geq 1\right)$, and quasineutrality is assumed to hold ( $\left.\alpha * 1\right)$, where $\alpha=1 / \mathrm{k}_{\mathrm{ia}} \lambda_{\mathrm{DE}}$ and $\lambda_{\mathrm{DE}}$ is the electron Debye length. The heat flux (q) can be inferred with some degree of accuracy from the relative drift measurements and since the electron temperature gradients $\left(\nabla \mathrm{T}_{\mathrm{e}}\right)$ can be deduced from the spatially dependent sound 
speed data, the electron thermal conductivity $\left(\kappa_{e}\right)$ may be measured from $\left(q=-\kappa_{e} \nabla T_{e}\right)$. The model also correctly predicts the appearance of a zero-frequency or entropy peak in the Thomson spectra in the fluid limit $\left(\mathrm{k}_{\mathrm{ia}} \lambda_{\mathrm{ii}} \rightarrow 0\right)$.

To summarize, this dissertation presents an experiment in which collective Thomson scattering is, for the first time, used at $266 \mathrm{~nm}$ to make detailed measurements of a laser-generated, collisional plasma. These detailed measurements are used to test the accuracy of the simulation code LASNEX and a simple analytical model. The versatility of Thomson scattering is expanded upon by extending existing theory and developing and analytic model to help analyze Thomson spectra from collisional laser-plasmas. This model allows new information to be obtained about the relative thermal flow velocity between the electrons and ions.

The dissertation is organized as follows: In Chapter 2 a discussion on existing collisional and collisionless Thomson scattering theory will be presented. The existing theory with some minor modifications will be appropriate for analyzing data from a collisional laser-plasma to obtain electron density, sound speed, and drift velocity measurements. Chapter 3 will describe in detail the experimental configuration used to measure the three quantities mentioned above. The results of the experiments are analyzed and discussed in Chapter 4. Chapter 5 presents a new theory for describing ion-ion collision related effects in Thomson scatiering. This new theory provides an analytic model for interpreting Thomson spectra from collisional laser-produced plasmas. Chapter 6 outlines a proposed experiment for testing and utilizing the theory in Chapter 5 to measure the relative thermal flow velocity between the electrons and ions in a collisional 
laser-produced plasma using Thomson scattering. Finally, the conclusions for the dissertation are presented in Chapter 7 along with some thoughts about the future. 
Chapter II: Thomson scattering theory - fluid and kinetic limits

This chapter will present an outline of the current theory of Thomson scattering as it applies to thermal level, ion-acoustic waves. The discussion will start with a derivation of general formulas that are applicable independent of collisions to any homogeneous, isotropic, two component (electrons and one ion species) plasma in quasiequilibrium. The requirements that the plasma be homogenous, isotropic, two component and in quasi-equilibrium is ultimately chosen to simplify the theory, but these requirements are not as stringent as they might appear. The homogeneous treatment is valid as long as the scattering volume from which Thomson scattering occurs is small enough compared to the scale lengths of density and temperature in the plasma. The plasma is isotropic if there are no magnetic fields present, and is approximately isotropic if the magnetic field strengths are small. The two component requirement is fulfilled if the plasma is fully ionized or if a large percentage of the distribution of ions have one charge state. Quasi-equilibrium requires that the electrons and ions in the plasma are in equilibrium with themselves, but not necessarily with each other. The unperturbed distribution functions for the electrons and ions will be Maxwellian and each species will have a respective temperature $T_{e}, T_{i}$, but the temperatures can be different $\left(T_{e} \neq T_{i}\right)$. From the general formulas, two limits of the theory will be shown for plasmas which are characterized in the fluid regime by collisional ions $\left(\mathrm{k}_{\mathrm{ia}} \lambda_{\mathrm{ii}} \rightarrow 0\right)$ and plasmas which are characterized in the kinetic regime by collisionless ions $\left(\mathrm{k}_{\mathrm{ia}} \lambda_{\mathrm{ii}} \rightarrow \infty\right)$. In both cases, the electrons are assumed collisionless $\left(\mathrm{k}_{\mathrm{ia}} \lambda_{\mathrm{ei}}, \mathrm{k}_{\mathrm{ia}} \lambda_{\mathrm{ee}}{ }^{* 1}\right)$. The collisionality represents the 
degree to which collisional effects are important for an ion-acoustic wave of wave number $\mathrm{k}_{\mathrm{ia}}$, and $\lambda_{e e}, \lambda_{\mathrm{ei}}, \lambda_{\mathrm{ii}}$ are the electron-electron, electron-ion, and ion-ion mean free paths as defined by Braginskii (Ref. 10), respectively,

$$
\begin{aligned}
& \lambda_{e e}=1.02 \times 10^{13} \frac{T_{e}^{2}}{n_{e} \ln \Lambda_{e e}} \quad(\mathrm{~cm}) \\
& \lambda_{e i}=1.44 \times 10^{13} \frac{T_{e}^{2}}{Z n_{e} \ln \Lambda_{e i}} \quad(\mathrm{~cm}) \\
& \lambda_{i i}=2.04 \times 10^{13} \frac{T_{i}^{2}}{Z^{3} n_{e} \ln \Lambda_{i i}} \quad(\mathrm{~cm})
\end{aligned}
$$

where the electron and ion temperatures $T_{e}, T_{i}$ are in $(e V)$ and the electron density $n_{e}$ is in $\left(\mathrm{cm}^{-3}\right)$. Although the laser-produced plasma studied for this dissertation lies in the intermediate collisional range for the ions $\left(\mathrm{k}_{\mathrm{ia}} \lambda_{\mathrm{ii}} \approx 1\right)$, it will be shown that the fluid and kinetic limits of Thomson theory are adequate for measuring the three quantities: electron density, sound speed, radial drift. However, the exact collisionality of the ion-acoustic waves does influence measurements that are sensitive to the shape of the Thomson spectrum, such as line width analysis. A theory will be presented that can quantitatively describe these effects in Chapter 5 .

When an optical probe of wave vector $\mathbf{k}_{\mathrm{pr}}$ and frequency $\omega_{\mathrm{pr}}$ is incident upon a plasma, Bragg scattering can occur off a plasma fluctuation $\left(k_{\mathrm{fl}}, \omega_{\mathrm{fl}}\right)$ and the resulting scattered light satisfies the conditions,

$$
\vec{k}_{s c}=\vec{k}_{p r}+\vec{k}_{f}
$$




$$
\omega_{s c}=\left(\omega_{p r} \pm \omega_{f f}\right)+\vec{k}_{f t} \cdot \vec{U}_{d r i t}
$$

where $\mathbf{k}_{\mathrm{sc}}$ and $\omega_{\mathrm{sc}}$ are the wave vector and frequency of the scattered light, respectively, and the effect of a nonstationary plasma $\mathbf{U}_{\text {drift }}$ on the frequency shift is added. The power scattered, $P_{s c}\left(k_{s c}, \omega_{s c}\right)$, in the direction $k_{s c}$, into solid angle $d \Omega$ and frequency interval $\omega_{s c}$ $\rightarrow \omega_{\mathrm{sc}}+\mathrm{d} \omega_{\mathrm{sc}}$ is given by ${ }^{109}$,

$$
P_{s c}\left(\vec{k}_{s c}, \omega_{s c}\right) d \Omega d \omega_{s c}=P_{p r} e^{-\Delta} \sigma_{\tau} n_{e} \cdot\left(\frac{V}{A}\right) \frac{S\left(\vec{k}_{f b} \omega_{f}\right)}{2 \pi} \mid \hat{k}_{s c} \times\left(\hat{k}_{s c} \times \hat{e}_{p r}\right)^{2} d \Omega d \omega_{s c}
$$

where $\mathrm{P}_{\mathrm{pr}}$ is the probe beam power with cross-sectional area $\mathrm{A}, \sigma_{\mathrm{T}}$ is the classical electron Thomson cross section $\left(7.952 \times 10^{-26} \mathrm{~cm}^{2}\right), \hat{\mathrm{k}}_{\mathrm{sc}}$ and $\hat{\mathrm{e}}_{\mathrm{pr}}$ are unit vectors, $\hat{\mathrm{e}}_{\mathrm{pr}}$ is along the probe beam polarization, $\mathrm{V}$ is the volume defined by the intersection of the probe beam and the collection field of view, and $S\left(k_{\mathrm{fl}}, \omega_{\mathrm{f}}\right)$ is the dynamical form factor. The factor $\mathrm{e}^{-\Delta}$ is included to represent possible absorption of the probe and scattered waves due to inverse bremsstrahlung in the plasma. The quantities in Eq. (13) are written in terms of their spatial and temporal Fourier transforms $(\mathbf{k}, \omega)$. This form is most convenient for working with the theory on Thomson scattering and will be used in the rest of this chapter unless otherwise stated.

The dynamical form factor $S(k, \omega)$ is the wave-vector/frequency-dependent part of the Thomson cross section and represents the effects of scattering due to electron motion as well as correlations between the electrons. The dynamical form factor is related to the mean square fluctuations in the electron density of the plasma and is given by (Ref. 109), 


$$
S(\vec{k}, \omega) \equiv \lim _{T \rightarrow \infty, V \rightarrow \infty} \frac{1}{T V}\left(\frac{\left|\delta n_{e}(\vec{k}, \omega)\right|^{2}}{n_{e}}\right)
$$

where $\mathrm{T}$ and $\mathrm{V}$ are the time and volume that the average is taken over. The dynamical form factor can be derived for a general homogenous, isotropic, two component plasma in quasi-equilibrium with the use of Coulomb's law, the continuity equation, Ohm's law, and the fluctuation-dissipation theorem $110,111,112,113,114$. The derivation for the dynamical form factor will involve solving for the response of the plasma to external perturbative fields in the presence of the self consistent fields.

The fluctuation-dissipation theorem provides a powerful tool for connecting the power spectrum of fluctuations of a system in thermodynamic equilibrium to the imaginary part of the relevant linear response function of the system. For example, in the Coulomb gauge with no charge density sources, the current density $\mathbf{j}$ and the vector potential A are related to one another through a wave equation (Ref. 8),

$$
\vec{\nabla}^{2} \vec{A}-\frac{1}{c^{2}} \frac{\partial^{2} \vec{A}}{\partial t^{2}}=-\frac{4 \pi}{c} \vec{j}
$$

The current density acts as a source for the vector potential in this equation and assuming a linear response $(\alpha)$, a relation may be written between the current density and the vector potential. The fluctuation-dissipation theorem then relates the mean square fluctuation of the vector potential to the linear response $(\alpha)$. The linear response and the mean square fluctuation of the vector potential can be written (Ref. 113), 


$$
\begin{gathered}
\vec{A}(\vec{k}, \omega)=\alpha(\vec{k}, \omega) \vec{j}(\vec{k}, \omega) \\
\left\langle A A_{j}\right\rangle_{\vec{k} \omega}=\frac{\hbar}{e^{\hbar \omega / T}-1} i\left[\alpha(\vec{k}, \omega)-\alpha^{*}(\vec{k}, \omega)\right] \approx \frac{T}{\omega} i\left[\alpha(\vec{k}, \omega)-\alpha^{*}(\vec{k}, \omega)\right]
\end{gathered}
$$

where the approximation holds for sufficiently high temperatures and the ${ }^{*}$ refers to the complex conjugate. The final step in deriving a general form for $\mathbf{S}(\mathbf{k}, \omega)$ will involve Eq. (16), but for now the derivation will start with Coulomb's law which for a medium with no free charge density is given by,

$$
i \vec{k} \cdot \vec{D}(\vec{k}, \omega)=i \vec{k} \cdot \vec{E}(\vec{k}, \omega)-4 \pi \rho_{\text {ind }}(\vec{k}, \omega)=0
$$

where $\mathbf{D}$ and $\mathbf{E}$ are the electric displacement and electric field vectors, respectively, and $\rho_{\text {ind }}$ is the induced charge density due to external perturbations. In the case of a plasma, neglecting the free charge density is warranted because a plasma by definition is charge neutral as long as $\mathrm{kL} * 1$, where $\mathrm{L}$ is the minimum of the plasma scalelengths. Along with Eq. (17) comes the following definitions,

$$
\begin{aligned}
\vec{D}(\vec{k}, \omega)= & \vec{E}(\vec{k}, \omega)+4 \pi \vec{P}(\vec{k}, \omega)=\epsilon(\vec{k}, \omega) \vec{E}(\vec{k}, \omega) \\
& \epsilon \vec{k}, \omega)=1+\sum_{j} \chi_{j}(\vec{k}, \omega) \\
& \rho_{\text {ind }}(\vec{k}, \omega)=-i \vec{k} \cdot \vec{P}(\vec{k}, \omega) \\
& \vec{j}_{\text {ind }}(\vec{k}, \omega)=i \omega \vec{P}(\vec{k}, \omega)
\end{aligned}
$$

where $\mathbf{P}$ is the polarization vector, $\epsilon$ is defined as the dielectric response function of the medium, $\chi_{\mathrm{j}}$ is the susceptibility of the $\mathrm{j}^{\text {th }}$ species in the plasma, and $\mathbf{j}_{\text {ind }}$ is the induced current density. The induced charge and current densities can be related through the continuity equation, 


$$
-i \omega \rho_{\text {ind }}(\vec{k}, \omega)+i \vec{k} \cdot \vec{j}_{\text {ind }}(\vec{k}, \omega)=0
$$

and Ohm's law for a non-magnetic, homogenous plasma is given by Eq. (2) as,

$$
\vec{j}_{\text {ind }}(\vec{k}, \omega)=\sigma \vec{E}(\vec{k}, \omega)=\frac{-i \omega(\epsilon-1)}{4 \pi} \vec{E}(\vec{k}, \omega)
$$

where the last equality is found from Eq.'s (17), (18), and (19). External perturbing fields $\mathbf{E}_{\text {ext }}{ }^{i}$ and $E_{\text {ext }}{ }^{e}$ which couple independently to the ions and electrons can be introduced into the plasma. These fields induce charge and current densities which in turn generate a self consistent electric field $\mathbf{E}_{\text {self }}$. This process can be written in terms of the induced current density for the electrons and ions through Eq. (20),

$$
\begin{aligned}
& \vec{j}_{\text {ind }}^{e}=\frac{-i \omega \chi_{e}}{4 \pi}\left(\vec{E}_{\text {self }}+\vec{E}_{\text {exx }}^{e}\right) \\
& \vec{j}_{\text {ind }}^{i}=\frac{-i \omega \chi_{i}}{4 \pi}\left(\vec{E}_{\text {self }}+\vec{E}_{\text {exx }}^{i}\right)
\end{aligned}
$$

The self consistent field can in turn be written in terms of the total induced charge density by combining Coulomb's law (Eq. (17)) and the continuity equation (Eq. (19)),

$$
\vec{E}_{\text {self }}=\frac{4 \pi}{i \omega}\left(\vec{j}_{\text {ind }}^{c}+\vec{j}_{\text {ind }}^{i}\right)=\frac{4 \pi}{i \omega} \vec{j}_{\text {ind }}^{\text {tot }}
$$

The self consistent field can be eliminated between equations (21) and (22), and the solution for the induced current densities and external fields is given by, 


$$
\begin{gathered}
\vec{j}_{\text {ind }}^{e}=\frac{i \omega}{4 \pi \epsilon}\left(-\chi_{e}\left(1+\chi_{i}\right) \vec{E}_{e x t}^{e}+\chi_{e} \chi_{i} \vec{E}_{e x t}^{i}\right) \\
\vec{j}_{\text {ind }}^{i}=\frac{i \omega}{4 \pi \epsilon}\left(-\chi_{i}\left(1+\chi_{e}\right) \vec{E}_{e x t}^{i}+\chi_{e} \chi_{i} \vec{E}_{e x t}^{e}\right) \\
\vec{E}_{\text {ext }}^{e}=\frac{-4 \pi}{i \omega} \frac{\vec{j}_{\text {ind }}^{e}}{\chi_{e}}-\frac{4 \pi}{i \omega} \vec{j}_{\text {ind }}^{\text {oot }} \\
\vec{E}_{\text {ext }}^{i}=\frac{-4 \pi}{i \omega} \frac{\vec{j}_{\text {ind }}^{i}}{\chi_{i}}-\frac{4 \pi}{i \omega} \vec{j}_{\text {ind }}^{\text {tor }}
\end{gathered}
$$

Since the external fields are assumed to act independently on the electrons and ions, the fluctuations of each field are only determined by the temperature of the appropriate subsystem. Therefore, the fluctuation-dissipation theorem Eq. (16) can be used with the results of Eq. (23) to separately find the mean square fluctuations of each external field due to the response of the induced current densities,

$$
\begin{gathered}
\left\langle E_{j}^{e} E_{k}^{e}\right\rangle=\frac{-8 \pi T_{e}}{\omega^{2}} \operatorname{Im}\left(\frac{1}{\chi_{e}}\right) \\
\left\langle E_{j}^{i} E_{k}^{l}\right\rangle=\frac{-8 \pi T_{i}}{\omega^{2}} \operatorname{Im}\left(\frac{1}{\chi_{i}}\right) \\
\left\langle E_{j}^{e} E_{k}^{i}\right\rangle=0
\end{gathered}
$$

where the last relation holds because the fields are assumed independent. The mean square fluctuation of the induced electron current density is now found by combining Eq. (23) and Eq. (24),

$$
\left\langle j_{j}^{e} j_{k}^{e}\right\rangle=\frac{2 \omega T_{e}}{4 \pi} \frac{\left|1+\chi_{i}\right|^{2}}{\mid \epsilon P^{2}} \operatorname{Im}\left(\chi_{e}\right)+\frac{2 \omega T_{i}}{4 \pi} \frac{\left|\chi_{e}\right|^{2}}{|\epsilon|^{2}} \operatorname{Im}\left(x_{i}\right)
$$

The induced current density is related to the induced charge density through the continuity equation Eq. (19). The induced charge density is then related to the induced electron 
density by $\left(\rho_{\text {ind }}=-\mathrm{e} \delta \mathrm{n}_{\mathrm{e}}\right)$. These relationships can be used with Eq. (14) and Eq. (25) to find the general form for the dynamical form factor in terms of the electron and ion susceptibilities of the plasma,

$$
S(\vec{k}, \omega)=\frac{2}{\omega \alpha^{2}}\left|\frac{\left(1+\chi_{i}\right)}{\epsilon}\right|^{2} \operatorname{Im}\left(\chi_{e}\right)+\frac{2 T_{i}}{\omega \alpha^{2} T_{e}}\left|\frac{\chi_{e}}{\epsilon}\right|^{2} \operatorname{Im}\left(\chi_{i}\right)
$$

where $\alpha \equiv 1 / k \lambda_{\mathrm{DE}}$, and $\lambda_{\mathrm{DE}}=\left(\mathrm{k}_{\mathrm{B}} \mathrm{T}_{\mathrm{e}} / 4 \pi \mathrm{n}_{\mathrm{e}} \mathrm{e}^{2}\right)^{1 / 2}$ is the electron Debye length. Equation (26) has been derived without using any assumptions about the collisional nature of the plasma. The dependence of $S(k, \omega)$ or the scattered frequency spectrum on the plasma conditions is included through the response functions $\chi_{\mathrm{e}}, \chi_{\mathrm{i}}$ of the electrons and ion species in the plasma and the parameter $\alpha$. Resonances appear in the scattered spectrum when the real part of the dielectric function approaches zero $(\operatorname{Re}(\epsilon) \rightarrow 0)$ in Eq. (26). The parameter $\alpha$ can be used as a qualitative gauge for whether the sharp resonances will appear or whether the scattered spectrum will contain only broad uncorrelated features. When $\alpha<1$, the scattered spectrum is typically due to noncollective effects in the plasma. Scattering occurs from electrons which exhibit uncorrelated, random, thermal motion and the width of the broad spectral feature in this case can be related to the electron temperature in the plasma. When $\alpha>1$, the scattering process is referred to as collective Thomson scattering. In this case, scattering occurs from electrons which are undergoing collective motion either in response to a low frequency ion-acoustic wave or from a high frequency electron plasma wave. As mentioned in Chapter I, this dissertation involves using collective Thomson scattering from electron motion induced by ionacoustic fluctuations. Therefore, particular attention is paid to the low frequency, $\alpha>$ 
1 results of the following sections.

The first step towards investigating the spectral features predicted by the dynamical form factor in Eq. (26), involves computing the electron and ion susceptibilities of the plasma. In general, the susceptibilities are found by the use of Ohm's law (Eq. (20)), the continuity equation (Eq. (19)), and the definitions for the electric field in terms of the scalar potential $(E=-i k \phi)$ and the induced charge density in terms of the perturbed density $\left(\rho_{\text {ind }}{ }^{\alpha}=\mathrm{Z}_{\alpha} \mathrm{e} \delta \mathrm{n}_{\alpha}\right)$,

$$
\chi_{\alpha}(\vec{k}, \omega)=\frac{-4 \pi Z_{\alpha} e}{k^{2}} \frac{\delta n_{\alpha}(\vec{k}, \omega)}{\phi_{e x z}(\vec{k}, \omega)}
$$

where $Z_{e}=-1$ for an electron. Unlike the argument leading to Eq. (21) which involved including a self consistent field, Eq. (27) results from only considering the direct coupling between an external potential and the induced change in density. The respouse of the electrons or ions under this situation is defined as the susceptibility of that given species in the plasma. The high frequency limit $(\omega \rightarrow \infty)$ of the susceptibility for the electrons and ions is in general zero $\left(\chi_{\alpha}(\mathbf{k}, \omega)=0\right)$ due to the fact the electrons and ions are massive and cannot respond at infinite frequencies. In the opposite, low frequency, D.C. limit $(\omega \rightarrow 0)$, the response of the electrons and ions is governed by Debye shielding. When a static point charge is inserted into the plasma, the electrons and ions will respond to eliminate the long range charge effects through Debye shielding. Since the electrons and ions are assumed individually to be in thermal equilibrium, their distribution functions are Maxwellian. When an external potential is introduced into the plasma that couples with either the electron or ion species, the energy of the species is increased by 
$\left(\mathrm{Z}_{\alpha} \mathrm{e} \phi_{\mathrm{ex}}\right)$ and the distribution function is given by,

$$
f_{O}^{\alpha}\left(v, \phi_{e x \alpha}\right)=\frac{n_{O}^{\alpha}}{\left(2 \pi \frac{k T_{\alpha}}{m_{\alpha}}\right)^{3 / 2}} e^{-\left(\frac{m_{\alpha} v^{2}}{2}+z_{\alpha} \phi_{c \alpha x}\right) / k T_{\alpha}}
$$

The perturbed density due to this external field can be found by integrating the difference between the distribution function with and without the applied potential over all velocity space,

$$
\delta n_{O}^{\alpha}=\int f_{O}^{\alpha}\left(v, \phi_{e x t}\right)-f_{O}^{\alpha}(v, 0) d^{3} v=n_{O}^{\alpha}\left(e^{\frac{-Z_{\alpha} e \phi_{e x t}}{k T_{\alpha}}}-1\right) \approx \frac{-Z_{\alpha} e n_{O}^{\alpha} \phi_{e x t}}{k T_{\alpha}}
$$

where the approximation holds for sufficiently high temperatures. The D.C. susceptibilities for the electrons and ions can now be found from Eq.'s (27) and (29),

$$
\begin{gathered}
\chi_{e}(\vec{k}, 0)=\alpha^{2} \\
\chi_{i}(\vec{k}, 0)=\frac{Z T_{e}}{T_{i}} \alpha^{2}
\end{gathered}
$$

The low and high frequency limits of the susceptibility for the electrons and ions can be used to calculate the total cross section $\mathrm{S}_{\mathrm{T}}(\mathbf{k})$ which is defined by,

$$
S_{T}(\vec{k})=\int_{-\infty}^{\infty} d \omega S(\vec{k}, \omega)
$$

Direct substitution of Eq. (26) into the integral is not possible because the expression in not analytic due to the $|\epsilon|^{2}$ factor in the denominator. This problem can be eliminated when the electron and ion temperatures are approximately equal $\left(T_{e} \approx T_{i}\right)$. In this case, 
Eq. (26) can be rewritten as,

$$
S(\vec{k}, \omega)=\frac{2}{\omega \alpha^{2}} \operatorname{Im}\left(\frac{\left(1+\chi_{i}\right) x_{e}}{1+\chi_{e}+\chi_{i}}\right)
$$

Since this function is analytic for $\operatorname{Im}(\omega) \geq 0$, the integration can now be performed along a closed contour in the upper half plane, indented on the real axis below the pole at zero. The result is given by (Ref. 109),

$$
S_{,}(\vec{k})=\frac{2 \pi\left(1+\chi_{i}(\vec{k}, 0)\right) \chi_{e}(\vec{k}, 0)}{\alpha^{2}\left(1+\chi_{e}(\vec{k}, 0)+\chi_{i}(\vec{k}, 0)\right)}=\frac{2 \pi\left(1+\alpha^{2}\left(Z T_{e} / T_{i}\right)\right)}{1+\alpha^{2}+\alpha^{2}\left(Z T_{e} / T_{i}\right)} \text { for } T_{e} \approx T_{i}
$$



Figure 1: Total cross section $\mathrm{S}_{\mathrm{T}}(\mathbf{k})$ for a general plasma as a function of $\mathrm{ZT}_{\mathrm{e}} / \mathrm{T}_{\mathrm{i}} \& \alpha$. 
where the D.C. results of Eq. (30) are used to obtain the last equality. The dependence of the total cross section on $\alpha$ for different values of $Z$ and $\left(T_{e}=T_{i}\right)$ is shown in Figure 1. The cross section is equal to 1 for $\alpha=0$ and approaches the limit $Z /(1+Z)$ for $\alpha \rightarrow \infty$. The total cross section in Eq. (33) applies to any homogeneous, isotropic, two component plasma in quasi-equilibrium independent of collisions, but unfortunately this is as far as the general approach can be taken. The fraction of the total cross section which is responsible for scattering in the low frequency or ion-acoustic feature as well as the exact form of the ion-acoustic spectrum can only be calculated from the specific equations that govern the evolution of the particular plasma of interest.

The exact form of the dynamical form factor was first derived independently by Fejer(1960) $)^{115}$, Renau(1960) $)^{116}$, Dougherty and Farley $(1960)^{117}$, and Salpeter $(1960)^{118}$ for a plasma in which the electrons and ions can be considered collisionless $\left(\mathrm{k}_{\mathrm{ia}} \lambda_{\mathrm{ii}}, \mathrm{k}_{\mathrm{ia}} \lambda_{\mathrm{ei}}, \mathrm{k}_{\mathrm{ia}} \lambda_{\mathrm{ee}} * 1\right)$. This subject has also been treated in review articles since that time. ${ }^{119,120}$ The evolution of the electron and ion distribution functions in a plasma which is collisionless is given by the Vlasov equation,

$$
\frac{\partial f_{\alpha}}{\partial t}+\vec{v} \cdot \vec{\nabla} f_{\alpha}+\frac{Z_{\alpha} e}{m_{\alpha}} \vec{E} \cdot \vec{\nabla}_{v} f_{\alpha}=0
$$

This equation can be linearized by expressing the electric field in terms of the potential $(E=-\nabla \phi)$, the distribution function as $f=f_{O}+f_{1} \exp (i(k z-\omega t))$, and the potential as $\phi=\phi_{\mathrm{O}}+\phi_{1} \exp (\mathrm{i}(\mathrm{kz}-\omega \mathrm{t}))$. The linearized Vlasov equations for the electrons and ions then become, 


$$
\begin{aligned}
& i\left(k v_{z}-\omega\right) f_{1}^{e}+i \frac{k e}{m_{e}} \phi_{1} \frac{\partial f_{O}^{e}}{\partial v_{z}}=0 \\
& i\left(k v_{z}-\omega\right) f_{1}^{i}-i \frac{Z k e}{m_{i}} \phi_{1} \frac{\partial f_{O}^{i}}{\partial v_{z}}=0 \\
& \text { where } f_{O}^{\alpha}=\frac{1}{\left(2 \pi v_{\alpha}^{2}\right)^{3 / 2}} e^{-\nu^{2} / 2 v_{\kappa}^{2}}
\end{aligned}
$$

where the background $\mathrm{f}_{\mathrm{O}}{ }^{\alpha}$ distribution is Maxwellian. The perturbed density which arises from the oscillating potential can now be solved for by rearranging the formulas in Eq. (35) for the perturbed distribution function $\mathrm{f}_{1}{ }^{\alpha}$ and integrating over velocity space,

$$
n_{1}^{\alpha}=\int f_{1}^{\alpha} d^{3} v=\frac{-Z_{\alpha} e \phi_{1}}{m_{\alpha} v_{\alpha}^{2}} \int \frac{k v f_{o}^{\alpha}}{k v_{z}-\omega} d^{3} v=\frac{-Z_{\alpha} e n_{o}^{\alpha} \phi_{1}}{T_{\alpha}}\left[1+\frac{\omega}{k v_{\alpha}} Z\left(\frac{\omega}{k v_{\alpha}}\right)\right]
$$

where the integral has been evaluated using the Landau prescription ${ }^{121}$ to incorporate the pole that occurs at $\omega=\mathrm{kv}_{\mathrm{z}}$, and $\mathrm{Z}\left(\omega / \mathrm{kv}_{\alpha}\right)$ is the plasma dispersion function. ${ }^{122}$ Equation (27) can now be used to find the susceptibilities for the electrons and ions from Eq. (36),

$$
\begin{gathered}
\text { Complex } \omega \quad \text { Real } \omega \\
x_{e}(\vec{k}, \omega)=\alpha^{2}\left[1+x_{e} Z\left(x_{e}\right)\right]=\alpha^{2} W\left(x_{e}\right) \\
x_{i}(\vec{k}, \omega)=\frac{Z T_{e}}{T_{i}} \alpha^{2}\left[1+x Z\left(x_{i}\right)\right]=\frac{Z T_{e}}{T_{i}} \alpha^{2} W\left(x_{i}\right) \\
\text { where } Z(x)=i \sqrt{\frac{\pi}{2}} e^{\frac{-x^{2}}{2}}\left[1+\operatorname{Erf}\left(\frac{i x}{\sqrt{2}}\right)\right] \\
W(x)=1-2 x e^{\frac{-x^{2}}{2}} \int_{0}^{x} e^{\frac{p^{2}}{2}} d p+i \sqrt{\frac{\pi}{2}} x e^{\frac{-x^{2}}{2}}
\end{gathered}
$$

where $x_{e}=\omega / k v_{e}$ and $x_{i}=\omega / k v_{i}$ are normalized frequencies. For complex frequencies, 
the susceptibilities are written in terms of a complex error function and for real frequencies, the susceptibilities posses a much simpler form in terms of the $W(x)$ function. When a frequency is written as a complex quantity, the real part corresponds to the mode frequency of the wave while the imaginary part is equal to the damping on that wave. For real frequency $\omega$, the dynamical form factor for a collisionless plasma is now written from Eq. (26) as,

$$
\begin{aligned}
& S(\vec{k}, \omega)\left|\begin{array}{c}
1+\frac{Z T_{e}}{T_{i}} \alpha^{2} W\left(x_{i}\right) \\
1+\alpha^{2} W\left(x_{e}\right)+\frac{Z T_{e}}{T_{i}} \alpha^{2} W\left(x_{i}\right)
\end{array}\right|^{2} \sqrt{2 \pi} \frac{e^{\frac{-x_{e}^{2}}{2}}}{k v_{e}}+ \\
& Z\left|\frac{-\alpha^{2} W\left(x_{e}\right)}{1+\alpha^{2} W\left(x_{e}\right)+\frac{Z T_{e}}{T_{i}} \alpha^{2} W\left(x_{i}\right)}\right|^{2} \sqrt{2 \pi} \frac{e^{\frac{-x_{i}^{2}}{2}}}{k v_{i}}
\end{aligned}
$$

The specific characteristics predicted by Eq.'s (37) and (38) can now be investigated as they apply to the ion-acoustic feature in Thomson scattering from a collisionless plasma. First, the damping mechanisms for ion-acoustic waves in a collisionless plasma are electron and ion Landau damping. The source of these damping mechan sms lies in the denominators of the terms in Eq. (38), that is really the dielectric function $\epsilon$ of the plasma. When the real part of $\epsilon$ goes to zero $(\operatorname{Re} \epsilon \rightarrow 0)$, a resonant peak will appear in the scattered spectrum. At the point where ( $\operatorname{Re} \epsilon \rightarrow 0$ ), the damping of the resonant wave is governed by the magnitude of the imaginary part of $\epsilon(\operatorname{Im} \epsilon)$, which is comprised of the imaginary parts of $W\left(x_{e}\right)$ and $W\left(x_{i}\right)$. Since $x_{e}$ is the frequency $\omega$ normalized to the electron thermal velocity, $\operatorname{Im~} \mathrm{W}\left(\mathrm{x}_{\mathrm{e}}\right)$ represents the electron Landau 
damping and likewise $\operatorname{Im} \mathrm{W}\left(\mathrm{x}_{\mathrm{i}}\right)$ represents ion Landau damping. For ion-acoustic waves $x_{i} \sim 1$ while $x_{e} * 1$. Electron Landau damping can be turned off by setting $x_{e}=0$ and then the effects of ion Landau damping can be studied. It can be shown that the electron Landau damping $\gamma_{\mathrm{e}}$ can be added afterwards to obtain the total damping on an ionacoustic wave through the factor,

$$
\gamma_{e}=\sqrt{\frac{\pi Z m_{e}}{8 m_{i}}} \operatorname{Real}\left(\omega_{i a}\right)
$$

where $\omega_{\mathrm{ia}}$ is the complex frequency of the ion-acoustic wave. Neglecting electron Landau damping $\left(x_{e}=0\right)$, the complex frequency for ion-acoustic waves can be solved for in the $\alpha \rightarrow \infty$ limit by utilizing the complex argument forms of the electron and ion susceptibilities in Eq. (37) and solving for the low frequency, complex root of $(\epsilon=1+$ $\chi_{\mathrm{e}}+\chi_{\mathrm{i}}=0$ ). Figure 2 shows the result of these calculations as a function of $\mathrm{ZT}_{\mathrm{e}} / \mathrm{T}_{\mathrm{i}}$. As can been seen from the figure, the mode frequency (Re $\omega)$ of the ion-acoustic wave increases with the ratio $\mathrm{ZT}_{\mathrm{e}} / \mathrm{T}_{\mathrm{i}}$ and the ion Landau damping (Im $\omega$ ) decreases quite rapidly from $\mathrm{ZT}_{\mathrm{e}} / \mathrm{T}_{\mathrm{i}}=1$ where it a considerable fraction of the mode frequency to $\mathrm{ZT}_{\mathrm{e}} / \mathrm{T}_{\mathrm{i}}=64$ where it is twelve orders of magnitude smaller than the mode frequency. For general $\alpha$, it can be shown that the mode frequency of the ion-acoustic wave as a function of $\mathrm{ZT}_{\mathrm{e}} / \mathrm{T}_{\mathrm{i}}$ and $\alpha$ is given by,

$$
\frac{\operatorname{Real}\left(\omega_{i a}\right)}{k_{i a} v_{i}}=\frac{c_{s}}{v_{i}}=\sqrt{\frac{Z T_{e}}{T_{i}}\left(\frac{\alpha^{2}}{\alpha^{2}+1}+\frac{3 T_{i}}{Z T_{e}}\right)}
$$

where $c_{s}$ is defined as the sound speed in the plasma and the factor of 3 is the adiabatic 




Figure 2: Real and imaginary parts of the complex ion-acoustic frequency as a function of $\mathrm{ZT}_{\mathrm{e}} / \mathrm{T}_{\mathrm{i}}$ for a collisionless plasma with $\alpha \rightarrow \infty$ neglecting electron Landau damping.

index or ratio of specific heats $\mathrm{C}_{\mathrm{P}} / \mathrm{C}_{\mathrm{V}}$ for a collisionless plasma. This is an approximate equation which is most accurate for large $\mathrm{ZT}_{\mathrm{e}} / \mathrm{T}_{\mathrm{i}}$ ratios.

The spectral characteristics of Thomson scattering from collisionless ion-acoustic waves can be analyzed through Eq. (38). This equation contains the complex $\mathrm{W}(\mathrm{x})$ function which is plotted in Figure 3. From the figure, it can be seen that the real part of $\mathrm{W}(\mathrm{x})$ attains a minimum value of -0.29 at $\mathrm{x} \approx 2.1$, and this functional property of $\mathrm{W}(\mathrm{x})$ can be used to qualitatively determine when ion-acoustic resonances will appear in a Thomson scattered spectrum. Ion-acoustic wave resonances occur in the spectrum given by $S(k, \omega)$ in Eq. (38), when the real part of the denominator of the expression goes to 


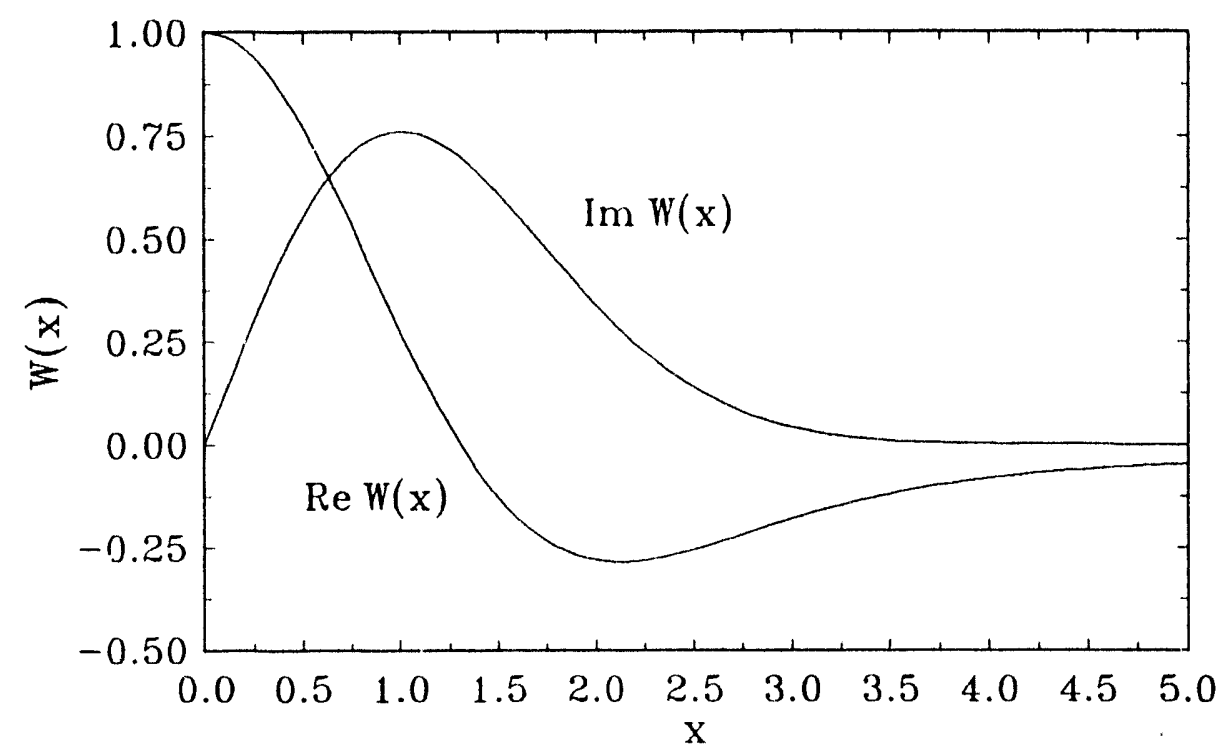

Figure 3: Real and imaginary parts of the function $W(x)$.

zero. Ignoring electron Landau damping effects for the moment, i.e. $x_{e}=0$ and $W\left(x_{e}\right)$ $=\alpha^{2}$, the real part of the denominator is given by $\left[1+\alpha^{2}+\left(\mathrm{ZT}_{\mathrm{e}} / \mathrm{T}_{\mathrm{i}}\right) \alpha^{2} \operatorname{Re} \mathrm{W}\left(\mathrm{x}_{\mathrm{i}}\right)\right]$. Since $\operatorname{Re} \mathrm{W}\left(\mathrm{x}_{\mathrm{i}}\right)>-0.29$, the following condition is qualitatively necessary for resonant peaks to occur in the ion-acoustic spectrum,

$$
\frac{\left(\frac{1+\alpha^{2}}{\alpha^{2}}\right)}{\frac{Z T_{e}}{T_{i}}} \leq 0.29
$$

This condition shows that when $\alpha * 1$, there will be no resonant peaks in the low frequency part of the Thomson spectrum for any value of $\mathrm{ZT}_{\mathrm{e}} / \mathrm{T}_{\mathrm{i}}$, and for increasing 


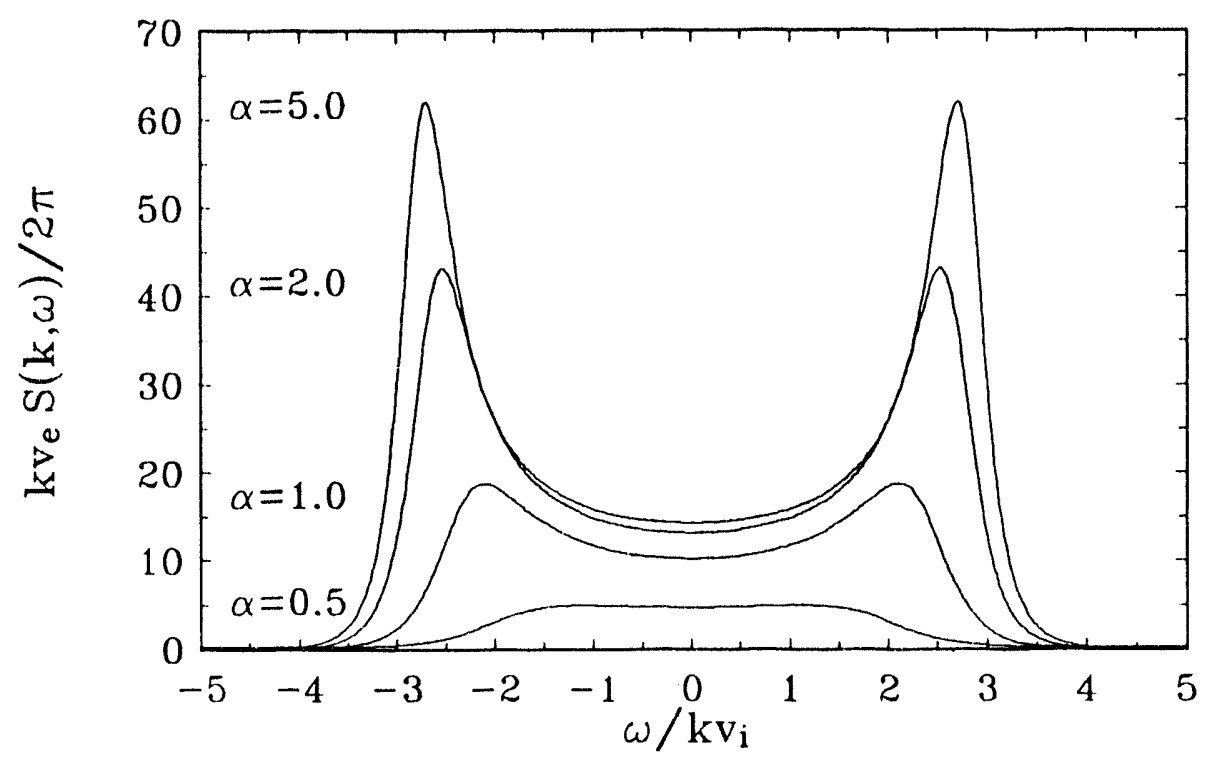

Figure 4: Theoretical ion-acoustic Thomson spectra for a collisionless aluminum plasma with $\mathrm{ZT}_{\mathrm{e}} / \mathrm{T}_{\mathrm{i}}=4$ and varying values of $\alpha$.

values of $\mathrm{ZT}_{\mathrm{e}} / \mathrm{T}_{\mathrm{i}}$, successively lower values of $\alpha$ are required to produce resonant peaks. In fact, for $\mathrm{ZT}_{\mathrm{e}} / \mathrm{T}_{\mathrm{i}}>8$ the requirement that $\alpha>1$ for collective Thomson scattering can be relaxed because resonant peaks will begin to appear in the spectrum for $\alpha<1$. The ramifications of this resonance condition can be seen by plotting the dynamical form factor in Eq. (38) for different values of $\mathrm{ZT}_{\mathrm{e}} / \mathrm{T}_{\mathrm{i}}$ and $\alpha$. Figure 4 shows ion-acoustic spectra for an aluminum plasma $\left(\mathrm{m}_{\mathrm{i}}=27 \mathrm{~m}_{\text {proton }}\right)$ with $\mathrm{ZT}_{\mathrm{e}} / \mathrm{T}_{\mathrm{i}}=4$ and varying values for $\alpha$, and as predicted by Eq. (41), the ion-acoustic feature comes into resonance as $\alpha$ increases. Figure 5 demonstrates the same principle but for increasing $\mathrm{ZT}_{\mathrm{e}} / \mathrm{T}_{\mathrm{i}}$ and fixed $\alpha$. As seen in the figures, when in resonance, the ion-acoustic feature for a collisionless 


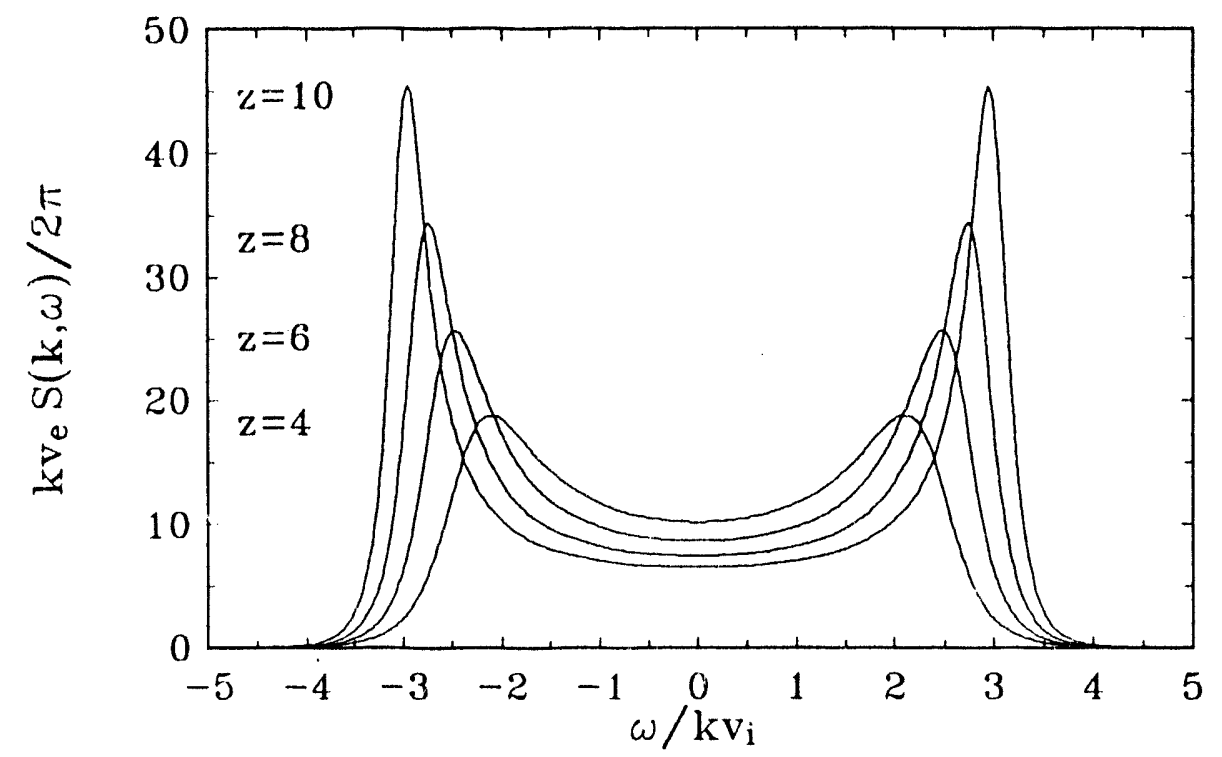

Figure 5: Theoretical ion-acoustic Thomson spectra for a collisionless aluminum plasma with $\alpha=1$ and varying values of $\mathrm{ZT}_{e} / \mathrm{T}_{\mathrm{i}}$.

plasma is comprised of two peaks. The two peaks result from scattering off of oppositely traveling ion-acoustic waves which have the same wave vector magnitude $\mathrm{k}_{\mathrm{ia}}$, but cause equal and opposite frequency shifts $\omega_{\mathrm{ia}}$ due to their opposing directions. The separation between the peaks is given by $2 \omega_{\mathrm{ia}}=2 \mathrm{k}_{\mathrm{ia}} \mathrm{c}_{\mathrm{s}}$ which is the ion-acoustic dispersion relation and the sound speed $c_{s}$ is given approximately by Eq. (40). Thus, the peak separation in a collective Thomson spectrum can be used to measure the sound speed in the plasma.

An additional feature is predicted by the dynamical form factor in Eq. (38). The peak heights become asymmetric when the thermal electrons drift relative to the thermal ions. An asymmetry results because the ion-acoustic wave that is traveling opposite to the 




Figure 6: Peak height asymmetries due to a relative drift between electrons and ions in an aluminum plasma with $Z \mathrm{ZT}_{\mathrm{e}} / \mathrm{T}_{\mathrm{i}}=8, \alpha=3$, and different values of $\mathrm{y}=\left(\mathrm{k}_{\mathrm{ia}} \cdot \mathrm{U}_{\mathrm{d}}\right) / \mathrm{kv}_{\mathrm{e}}$.

electron drift encounters enhanced electron Landau damping while the ion-acoustic wave traveling with the drift experiences a reduction in electron Landau damping. A driving mechanism for this phenomena is the return current due to heat transport in the plasma. A detailed discussion of this topic will be presented in Chapters 5 and 6, therefore, only a qualitative picture will be drawn in this section. A relative drift between the thermal electrons and ions can be examined by replacing the normalized frequency $x_{e}$ in Eq. (38) with $x_{e}+\left(k_{i} \bullet U_{d}\right) / k v_{e}$, where $U_{d}$ is the directional drift relative to the wave vector $k_{i a}$ of the ion-acoustic waves. The results from this analysis are shown in Figure 6 for an aluminum plasma with $\mathrm{ZT}_{\mathrm{e}} / \mathrm{T}_{\mathrm{i}}=8, \alpha=3$, and various values of $\mathrm{y}=\left(\mathrm{k}_{\mathbf{b}} \cdot \mathrm{U}_{\mathrm{d}}\right) / \mathrm{kv} \mathrm{e}_{\mathrm{e}}$. 
The effect of collisions in a fully ionized plasma on the dynamical form factor has been considered by several authors. Typical approaches include: the use of a FokkerPlanck type collision term with the Vlasov equation (Eq. (34)) to derive a collisional dependent form factor ${ }^{123,124,125}$, a quantum-mechanical treatment of the problem $^{126}$, and the use of the fluid equations to derive the collisional dependent form factor. ${ }^{127}$ Besides providing a theoretical model, reference 127 also provides experimental results of collective Thomson scattering for the ion-acoustic feature in a collisional plasma. The dynamical form factor derived from the fluid equations fits the data very well. Therefore, a similar but simplified approach will be used in this section to investigate the effects of collisions on the ion-acoustic feature obtained from Thomson scattering in a plasma where the ions are collisional $\left(\mathrm{k}_{\mathrm{ia}} \lambda_{\mathrm{ii}} * 1\right)$ and the electrons are assumed collisionless $\left(\mathrm{k}_{\mathrm{ia}} \lambda_{\mathrm{ei}}, \mathrm{k}_{\mathrm{ia}} \lambda_{\mathrm{ee}}\right.$ "1).

Since the ions are considered collisional $\left(\mathrm{k}_{\mathrm{ia}} \lambda_{\mathrm{ii}} * 1\right)$, the fluid equations can be used to describe the evolution of the ion species in the plasma. The fluid equations for the ions are given by (Ref. 10),

$$
\begin{gathered}
\frac{\partial n_{i}}{\partial t}+\vec{\nabla} \cdot\left(n_{i} \vec{v}_{i}\right)=0 \\
m_{i} n_{i} \frac{\partial \vec{v}_{i}}{\partial t}+m_{i} n_{i} \overrightarrow{v_{i}} \cdot \vec{\nabla} \vec{v}_{i}=-\vec{\nabla} p_{i}-\vec{\nabla} \cdot \vec{\pi}_{i}-Z_{e n} \vec{\nabla} \phi_{i}+\vec{R}_{e i} \\
\frac{3}{2} n_{i} \frac{\partial T_{i}}{\partial t}+\frac{3}{2} n_{i} \vec{v}_{i} \cdot \vec{\nabla} T_{i}+p_{i} \vec{\nabla} \cdot \vec{v}_{i}=-\vec{\nabla}_{q_{i}}-\vec{\pi}_{i}: \vec{\nabla} \vec{v}_{i}+Q_{e i} \\
\text { where } \quad p_{i}=n_{i} T_{i} \\
\text { closure } \quad \vec{q}_{i}=-\kappa_{i} \vec{\nabla} T_{i} \quad \vec{\pi}_{i}=-\frac{4}{3} \mu_{i} \vec{\nabla} \vec{v}_{i}
\end{gathered}
$$

where $p_{i}, \pi_{i}, q_{i}$ are the ion pressure, stress tensor, and heat flux, respectively. The linear 
closure relations have also been included which relate the heat flux and stress tensor to gradients in the temperature and velocity through the thermal conductivity $\kappa_{i}$ and the viscosity $\mu_{\mathrm{i}}$, respectively. The momentum and heat exchange coefficients $R_{\mathrm{ei}}, \mathrm{Q}_{\mathrm{ei}}$ between the electrons and ions will be neglected since the electrons are assumed collisionless. The fluid equations can be linearized in the same manner as the Vlasov equation in the previous section and are given by,

$$
\begin{gathered}
-i \omega n_{1}+i k n_{o} v_{1}=0 \\
-i \omega m n_{o} v_{1}=-i k T_{o} n_{1}-i k n_{o} T_{1}-i k \pi_{1}-i Z e k n_{o} \phi_{1} \\
-\frac{3}{2} i \omega n_{o} T_{1}+i k n_{o} T_{o} v_{1}=-i k q_{1} \\
\text { closure } \quad q_{1}=-\kappa^{o} \frac{n_{o} v_{i}}{k} i k T_{1} \\
\pi_{1}=-\frac{4}{3} \mu^{o} \frac{m n_{o} v_{i}}{k} i k v_{1}
\end{gathered}
$$

where the subscript $i$ for the ions has been assumed except for $v_{i}=\left(T_{i} / m_{i}\right)^{1 / 2}$, which is the ion thermal velocity, the subscripts $O$ and 1 refer to the background and perturbed values of the quantities, respectively, and the normalized transport coefficients are defined by, $\kappa^{\mathrm{o}}=3.9 \mathrm{k} \lambda_{\mathrm{ii}}$ and $\mu^{\mathrm{o}}=0.96 \mathrm{k} \lambda_{\mathrm{ii}}$. The system of formulas in Eq. (43) can be solved for the ratio $\left(n_{\mathrm{i}} / \phi_{\mathrm{i}}\right)$ which in turn can be used in Eq. (27) to find the ion susceptibility for a plasma with fluid ions,

$$
\chi_{i}=-\frac{4 \pi Z e}{k^{2}} \frac{n_{1}}{\phi_{1}}=-\frac{Z T_{e}}{T_{i}} \alpha^{2} \frac{x_{i}+\frac{2}{3} i \kappa^{o}}{x_{i}^{3}+\frac{2}{3} i\left(2 \mu^{o}+\kappa^{o}\right) x_{i}^{2}-\frac{1}{3}\left(5+\frac{8}{3} \mu^{o} \kappa^{o}\right) x_{i}-\frac{2}{3} i \kappa^{o}}
$$

where $x_{i}=\omega / k v_{i}$ is the normalized frequency. Since the electrons are assumed 


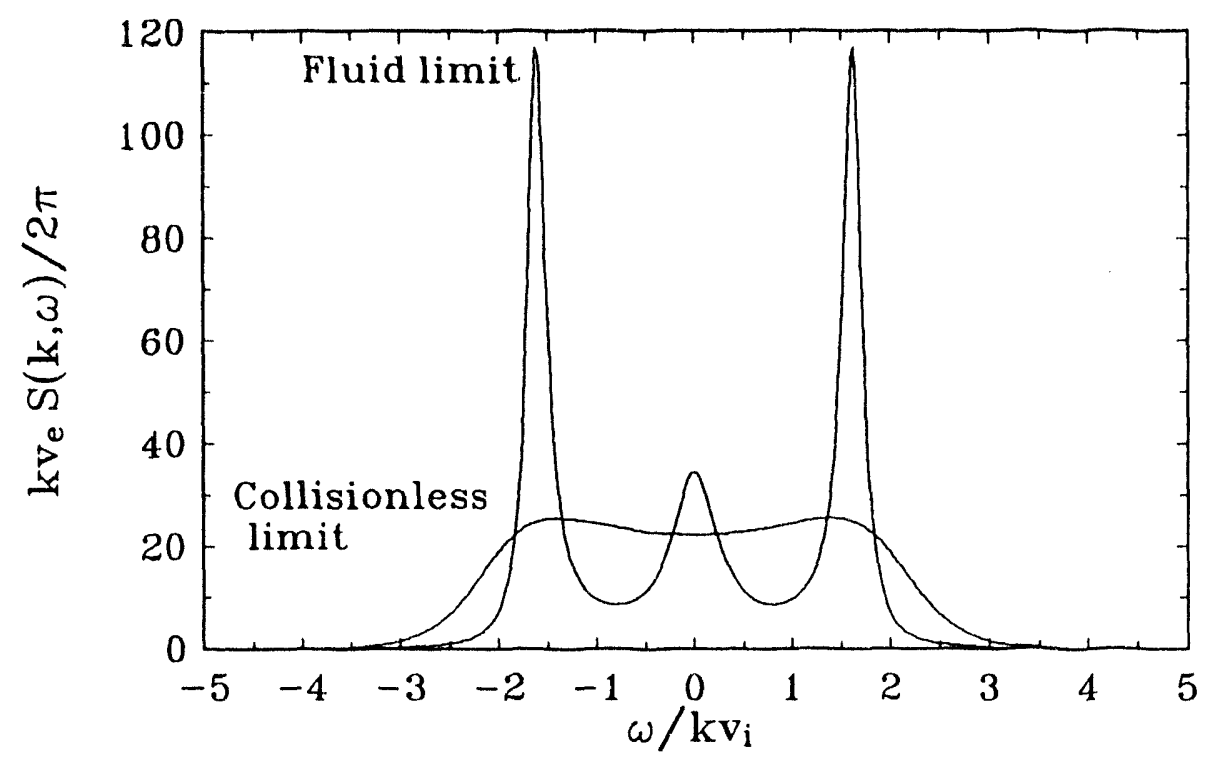

Figure 7: Theoretical ion-acoustic Thomson spectra in the ion fluid $\left(\mathrm{k}_{\mathrm{ia}} \lambda_{\mathrm{ii}}=0.1\right)$ and ion collisionless limits for a plasma with $\mathrm{ZT}_{\mathrm{e}} / \mathrm{T}_{\mathrm{i}}=1$ and $\alpha=300$.

collisionless, the electron susceptibility from Eq. (37) can be used with the fluid ion susceptibility of Eq. (44) to obtain the dynamical form factor for this ion collisional limit. Figure 7 shows the effects of ion-ion collisions on the low frequency ion-acoustic spectrum where the dynamical form factor in the fluid $\left(\mathrm{k}_{\mathrm{ia}} \lambda_{\mathrm{ii}}=0.1\right)$ and collisionless limits is plotted for identical plasma conditions, $\mathrm{ZT}_{\mathrm{e}} / \mathrm{T}_{\mathrm{i}}=1$ and $\alpha=300$. As can be seen by Figure 7, resonant peaks can appear in the collisional limit when they are barely visible in the collisionless limit under the same conditions. The resonant peaks emerge due to a reduction in the ion Landau damping of the ion-acoustic waves by ion-ion collisions which disturb the wave particle Landau resonance. The peak separation as in 
the collisional limit is given by $2 \omega_{i a}=2 k_{i a} c_{s}$, but the sound speed $\left(c_{s}\right)$ is modified due to the ion-ion collisions. It can be shown that the sound speed in the collisional limit $\left(k_{\mathrm{ia}} \lambda_{\mathrm{ii}} \rightarrow 0\right)$ is given exactly by,

$$
\frac{\operatorname{Real}\left(\omega_{k_{a}}\right)}{k_{i a} \nu_{i}}=\frac{c_{s}}{v_{i}}=\sqrt{\frac{Z T_{e}}{T_{i}}\left(\frac{\alpha^{2}}{\alpha^{2}+1}+\frac{5 T_{i}}{3 Z T_{e}}\right)}
$$

where the adiabatic index is now $5 / 3$ instead of 3 as in the collisionless case, Eq. (40). The presence of a zero frequency or entropy wave is also predicted in the fluid limit as shown in Figure 7. This wave is due to thermal level ion temperature fluctuations in the plasma which are balanced by ion density fluctuations to produce no pressure gradients. Since there are no pressure gradients, the wave does not propagate and has zero mode frequency. The entropy wave is present in the ion-acoustic spectrum only in the fluid limit, and will be discussed in more detail in Chapter 5 and Chapter 6 .

Based on the preceding results it can be shown that the fluid and kinetic limits of the Thomson scattering theory are adequate for measuring the three quantities: electron density, sound speed, and radial drift. The electron density can be measured from the ionacoustic Thomson spectrum by first integrating Eq. (13) over the low frequency part of the spectrum which includes the ion feature,

$$
\int P_{s c}\left(\vec{k}_{s c}, \omega_{s c}\right) d \omega_{s c} d \Omega=P_{s c}\left(\vec{k}_{s c}\right) d \Omega=P_{p r} e^{-\Delta} \sigma_{T^{n}} e\left(\frac{V}{A}\right) \frac{S_{T}^{i}\left(\vec{k}_{i a}\right)}{2 \pi} \mid \hat{k}_{s c} \times\left(\hat{k}_{s c} \times \hat{e}_{p r}\right)^{2} d \Omega(46)
$$

where $S_{T}{ }^{i}(k)$ is the total ion feature cross section. For $T_{e} \approx T_{i}$, the ion cross section in Eq. (46) will always be less than or equal to the total cross section predicted by Eq. (33) independent of the collisionality of the plasma. For the collisionless and fluid limits, the 


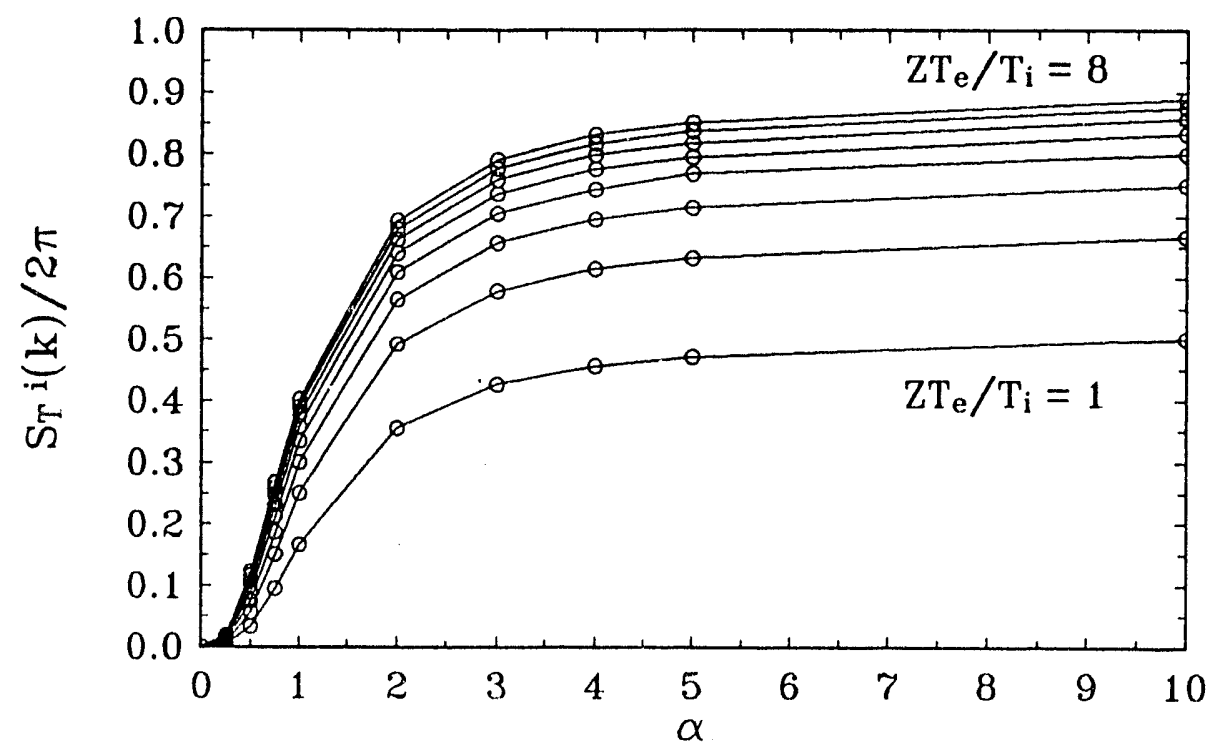

Figure 8: Total ion cross section $\mathrm{S}_{\mathrm{T}}{ }_{\mathrm{T}}(\mathbf{k})$ in the collisionless (line) and fluid (circle) ion limits as a function of $\alpha$ for $\mathrm{ZT}_{\mathrm{e}} / \mathrm{T}_{\mathrm{i}}$ ratios $(1 \rightarrow 8)$ in an aluminum plasma.

total ion cross section can be theoretically computed by integrating the appropriate dynamical form factors over low frequencies. Figure 8 shows the ion cross section in both the collisionless and fluid limits as a function of $\alpha$ for various $\mathrm{ZT}_{\mathrm{e}} / \mathrm{T}_{\mathrm{i}}$ ratios in an aluminum plasma. The solid lines are the collisionless results and the circles are the fluid results. As shown by the figure, the ion cross section approaches zero for $\alpha \rightarrow 0$ and a constant for $\alpha \rightarrow \infty$ and is the same in both limits for a given $\alpha$ and $\mathrm{ZT}_{\mathrm{e}} / \mathrm{T}_{\mathrm{i}}$ ratio. Therefore, even though the spectral distribution of the ion-acoustic feature can be markedly different in the two limits as seen in Figure 7, the total ion cross sections are the same! Since the ion cross section is identical in the fluid and collisionless limits, it 
will be assumed a constant for all values of ion collisionalicy $\left(\mathrm{k}_{\mathrm{ia}} \lambda_{\mathrm{ii}}\right)$. With a constant ion cross section, the electron density can be measured with some degree of accuracy by using Eq. (46) and Figure 8 with a relative intensity calibrated experimental collection system. The collection system and the exact method from which the electron density is . calculated will be described in detail in Chapter 3.

The sound speed varies from the fluid limit to the collisionless limit as shown by Eq.'s (40) and (45). This variation is due to the collisionally dependent adiabatic index which changes from $5 / 3$ in the fluid ions to 3 for collisionless ions. Knowing the exact value for the adiabatic index is crucial for calculating an accurate sound speed for low $\mathrm{ZT}_{\mathrm{e}} / \mathrm{T}_{\mathrm{i}}$ ratios, but as this ratio increases the value of the adiabatic index becomes less important. In Chapter 4, it will be shown that the experimental conditions for this dissertation fall in the range, $3 \leq \alpha \leq 15$ and $5 \leq \mathrm{ZT}_{\mathrm{e}} / \mathrm{T}_{\mathrm{i}} \leq 8$. The difference in the sound speed between the fluid and collisionless limits under these conditions is no more than $10 \%$. Values of $\mathrm{k}_{\mathrm{ia}} \lambda_{\mathrm{ii}}$ for the experiments are always less than one $\left(\mathrm{k}_{\mathrm{ia}} \lambda_{\mathrm{ii}}<1\right)$. The ion-acoustic waves measured are fairly collisional and choosing the adiabatic index $=5 / 3$ will introduce an error in the sound speed measurements much less than $10 \%$.

Finally, the term $\mathbf{k}_{\mathrm{ia}} \cdot \mathrm{U}_{\text {drift }}$ in Eq. (12) is added to take into account the effect of a nonstationary plasma on the frequency shifts in a Thomson spectrum. For the ionacoustic feature, a nonstationary plasına will shift the entire spectrum by a frequency amount determined by $\mathbf{k}_{\mathrm{ia}} \cdot \mathbf{U}_{\text {drift }}$. This Doppler shift will be used to measure the radial drift velocity of the plasma under investigation in this dissertation. Since Eq. (12) represents the conservation of energy in the scattering process and therefore has no 
dependence on the collisionality $\left(\mathrm{k}_{\mathrm{ia}} \lambda_{\mathrm{ii}}\right)$, the radial drift measurements are not effected by the collisionality of the plasma either.

In conclusion, this chapter has presented an outline of the current theory of Thomson scattering as it applies to thermal level, ion-acoustic waves. General formulas for the phase matching conditions of the Thomson scattering process and the Thomson scattered power are given. The general formula for the dynamical form factor which provides the spectral distribution of scattered light has been derived from first principles and is applicable to all homogeneous, isotropic, two component plasmas in quasiequilibrium independent of collisions. The dynamical form factor can be written in the collisionless $\left(\mathrm{k}_{\mathrm{ia}} \lambda_{\mathrm{ii}} \rightarrow \infty\right)$ and fluid $\left(\mathrm{k}_{\mathrm{ia}} \lambda_{\mathrm{ii}} \rightarrow 0\right)$ limits for the ions using the Vlasov and fluid equations, respectively. It has been shown that the predictions made by the two limiting forms of the dynamical form factor are adequate for use in measuring the electron density, sound speed, and radial drift in a plasma for which the ions lie in between the two limits of collisionality $\left(\mathrm{k}_{\mathrm{ia}} \lambda_{\mathrm{ii}} \approx 1\right)$. However, the exact form of the dynamical form factor for plasmas with intermediate collisional ions is needed for measurements relying on line shape analysis of the ion-acoustic spectrum. A theory for this intermediate collisional regime will be presented in Chapter 5 . 
Chapter III: Experimental configuration

This chapter will explain in detail the collective Thomson scattering experimental configuration used for obtaining two-dimensional electron density, sound speed, and radial drift profiles from a collisional laser plasma. The production of the diagnostic and plasma generating beams will be discussed along with the collection system used for monitoring collective Thomson scattering from the plasma. The Thomson-scattered signal is small and noise reduction techniques are used to reduce stray light from the diagnostic beam in order to increase the detectability of the Thomson-scattered signal. Since measurements of the laser plasma are made on a single point basis and two-dimensional information is required, the laser plasma generated for these experiments must be reproducible in the quantities that are being measured. Therefore, the laser pulse that generates the plasma in the experiment will have excellent energy stability, and smooth temporal and uniform spatial characteristics. The target used for plasma generation will need to have a smooth surface and be rotated to provide fresh target material for each laser shot. This combination of laser and target characteristics are sufficient to provide a plasma with reproducible electron density, sound speed, and radial drift profiles. The signal-to-noise in the collected data is improved by averaging multiple data shots at a given point in the plasma. The rate of data collection is enhanced by the high, $10 \mathrm{~Hz}$, repetition rate of the system. Collecting data at this rate requires carefully integrated timing of all of the components in the system, which will also be outlined in this chapter.

The experimental configuration is shown in Figure 9, Figure 10, and Figure 11. 




Figure 9: Experimental configuration; top view.

As seen in Figure 9, a Q-switched Nd:YAG laser (Spectra Physics Model DCR-3) operating at $10 \mathrm{~Hz}$ produces the optical pulses for both plasma generation and the Thomson diagnostic beam. The Nd:YAG laser possesses three features that improve the reproducibility of plasma generation. First, the pulse-to-pulse energy stability of the laser is $\pm 1 \%$ at $1.06 \mu \mathrm{m}$ which will provide reproducible energy deposition on target for producing the plasma. Second, without intervention, a Q-switched laser cavity will lase in several axial modes each separated by the characteristic frequency of the cavity, $\Delta v$ $=\mathrm{c} / 2 \mathrm{~L}$, where $\mathrm{L}$ is the cavity length and $\mathrm{c}$ is the speed of light. These axial modes will beat with each other in time and produce structure in the temporal profile of the laser pulse as seen in Figure 12. The mode beating structure is not reproducible from shot to 


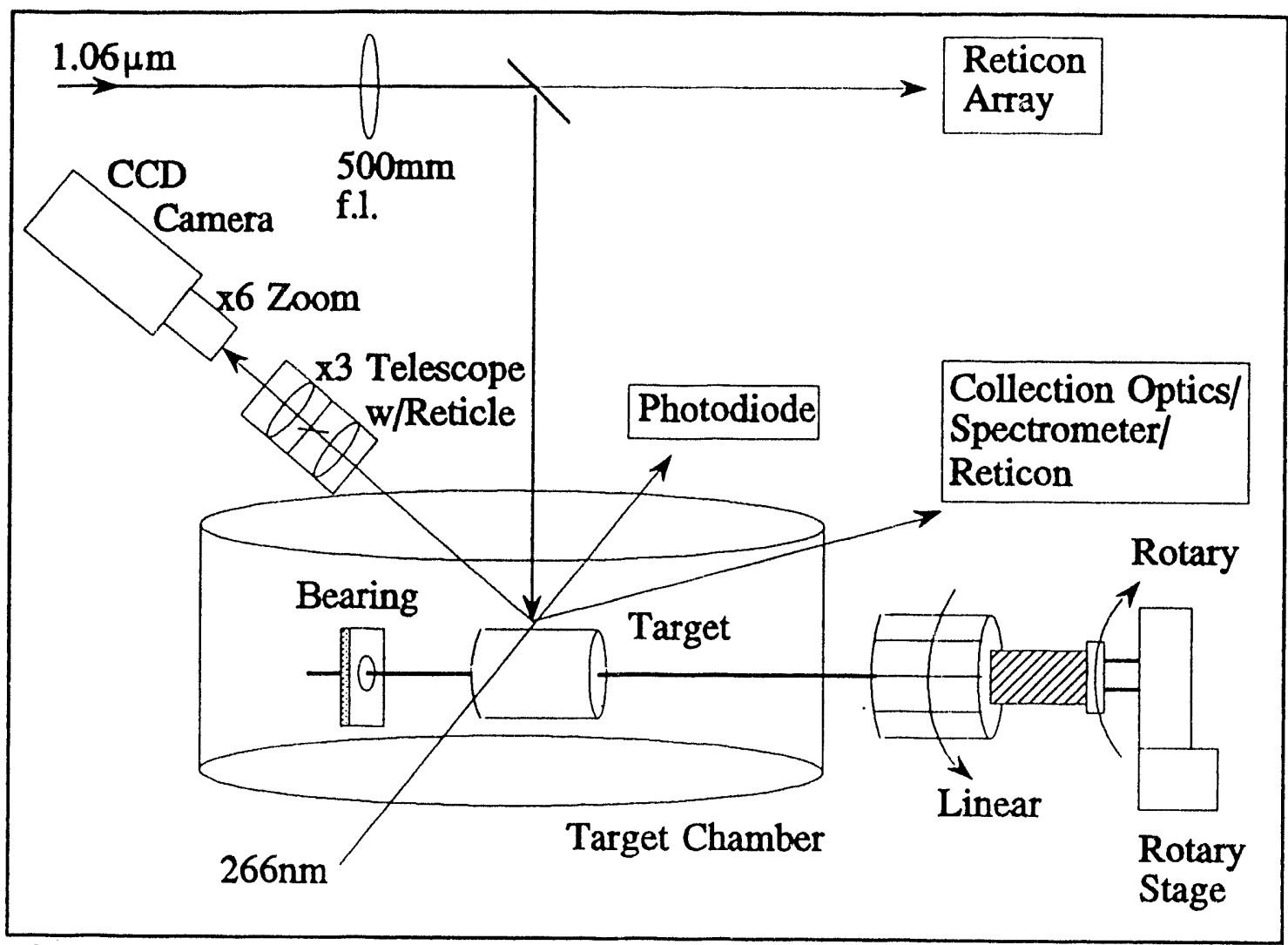

Figure 10: Experimental configuration; side view.

shot and therefore will hamper reproducible plasma generation. The mode beating can be eliminated by injecting a narrow linewidth, less than $\Delta v$, beam into the laser cavity. The energy of the injected beam is far above the photon noise of the cavity when the Q-switch is fired, thus lasing occurs only in the axial mode whose frequency coincides with that of the injected beam. The temporal profile of the single axial mode pulse is shown in Figure 13 (solid line). The narrow linewidth pulse $\left(0.003 \mathrm{~cm}^{-1}\right)$ is near transform limited in time (Full width at half maximum $(\mathrm{FWHM})=9 \mathrm{~ns})$. A Gaussian profile of the same FWHM width (dashed line) is shown in the figure for comparison. As can be seen by the comparison, the temporal profile of the laser pulse is not quite Gaussian, but it is smooth and reproducible. The data for both Figure 12 and Figure 13 are taken with an ANTEL 




Figure 11: Experimental configuration; side view of target.

Model AR-S2 photodiode (35ps rise time) connected to a $500 \mathrm{MHz}$ transient digitizer with the injection seeter of the laser blocked and unblocked, respectively. Finally, as with the axial modes, lasing will occur in several transverse modes of the standard hard edged resonator design for an unstable Q-switched cavity. ${ }^{128} \mathrm{~A}$ cross-section of the near-field spatial profile of another (Spectra Physics Model DCR-3) in the laboratory with a hard edged resonator cavity is shown in Figure 14. The profile consists of an annular ring, denoted by the outside peaks in the figure, with a central "spot of Arago", see Ref. 128. The target will not be uniformally irradiated by this beam. The near-field spatial profile of the laser can be improved by replacing the hard edged output coupler of the resonator with a variable reflectivity mirror (VRM). ${ }^{129,130,131}$ The laser used for the 


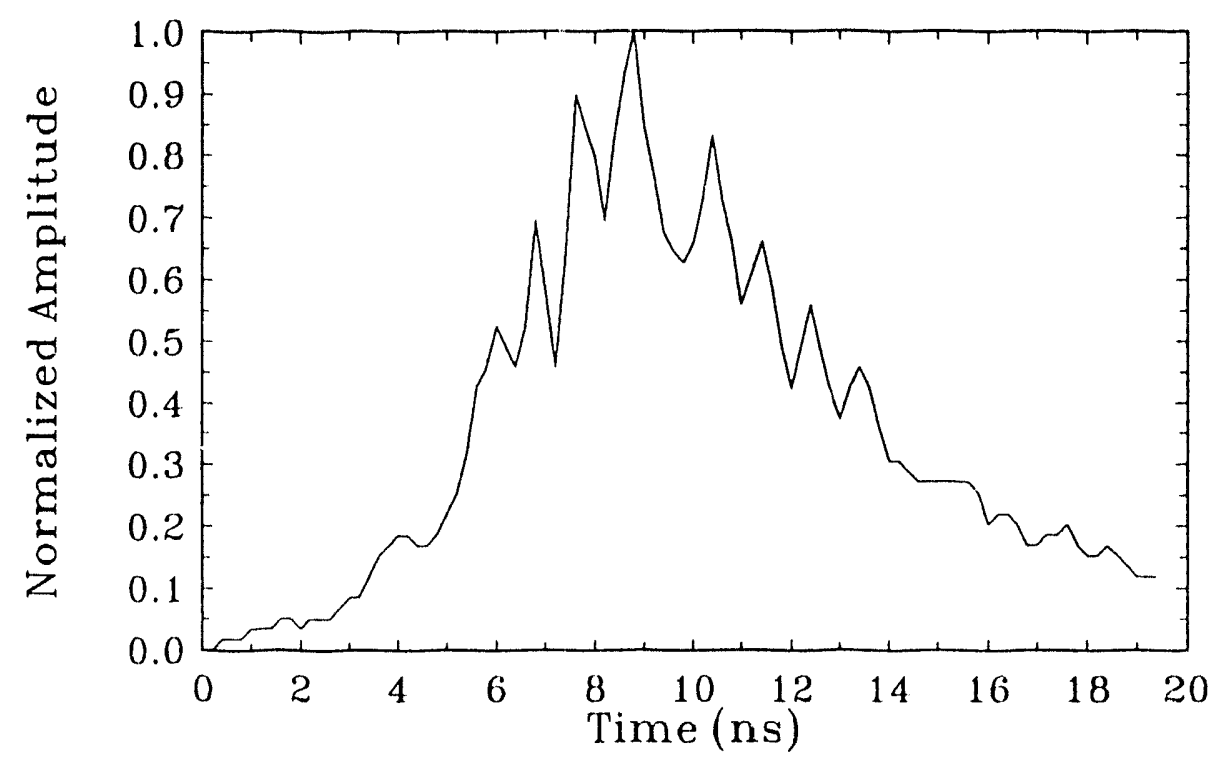

Figure 12: Temporal profile of the Nd:YAG laser pulse during unseeded operation. experiments described in this dissertation is equipped with a VRM. As seen by Figure 15, the near-field beam cross-section (solid line) is much more Gaussian in nature than with the hard edged resonator of Figure 14. The FWHM of the best fit Gaussian to the VRM profile (dashed line) is $8.2 \mathrm{~mm}$. Unfortunately, the spatial profile in Figure 15 is not entirely smooth due to diffraction effects resulting from over filling of the oscillator and amplifier rods in the laser. The next section will show how the diffraction affects the size of the focal spot obtained on target. The data in Figure 14 and Figure 15 is taken with an EG\&G 1024 element reticon array (25 $\mu \mathrm{m}$ pixel spacing) and a digital sampling oscilloscope.

As seen in Figure 9, the $1.06 \mu \mathrm{m}$ output from the laser is frequency doubled in 


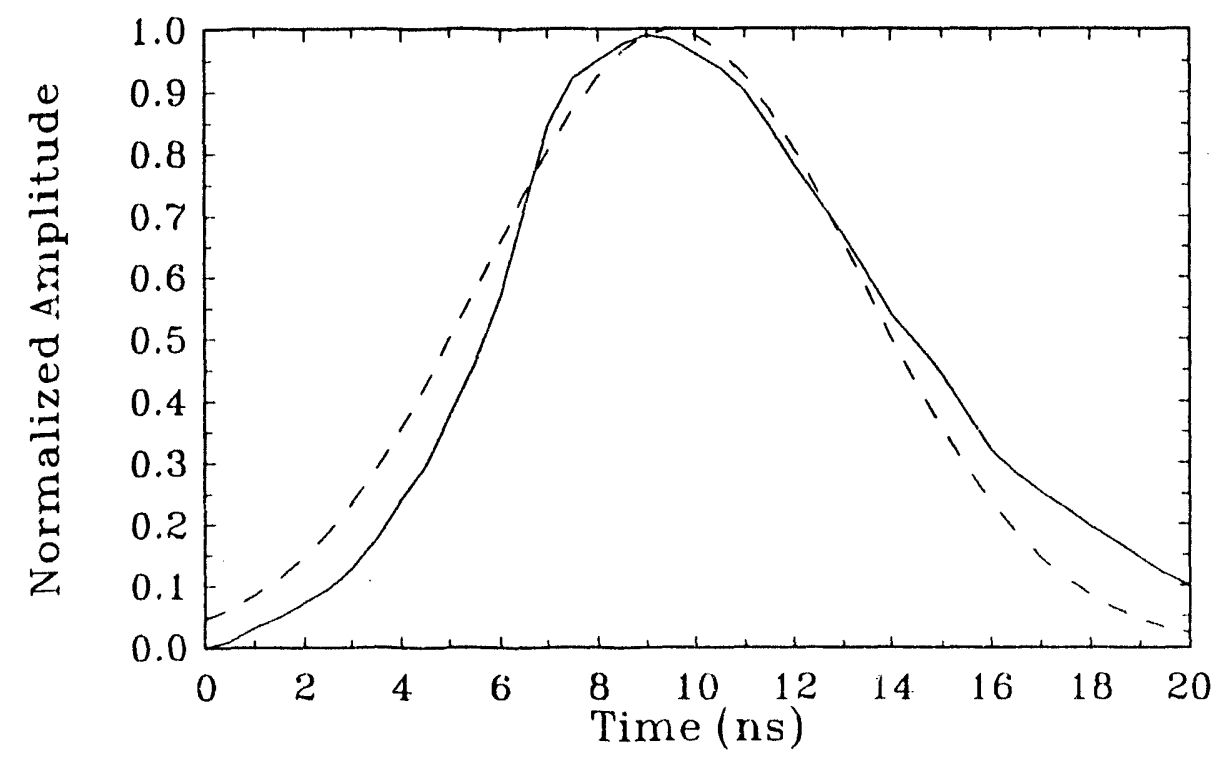

Figure 13: Temporal profile of the Nd:YAG laser pulse during seeded operation (solid line) with a Gaussian pulse shape of the same (FWHM $=9$ nsec) width for comparison (dashed line).

KDP providing $375 \mathrm{~mJ}$ of $532 \mathrm{~nm}$ light for diagnostics and $200 \mathrm{~mJ}$ of residual $1.06 \mu \mathrm{m}$ radiation for plasma generation. The plasma is preformed by focusing the $1.06 \mu \mathrm{m}$ light with a $500 \mathrm{~mm}$ focal length, anti-reflection coated lens through the top of the target chamber onto a disk target of aluminum, Figure 10. The aluminum target is one inch long, $3 / 4$ inch in diameter and machined on a lathe to provide a smooth surface finish. After machining, the target is promptly placed under vacuum in the target chamber to reduce oxidation of the aluminum surface. As shown by Figure 10, the target is rotated to a new surface for each laser shot by a rotary stage (AEROTECH Model 50SMB2-HM) which is connected to the target through a linear/rotary vacuum feedthru. When a 


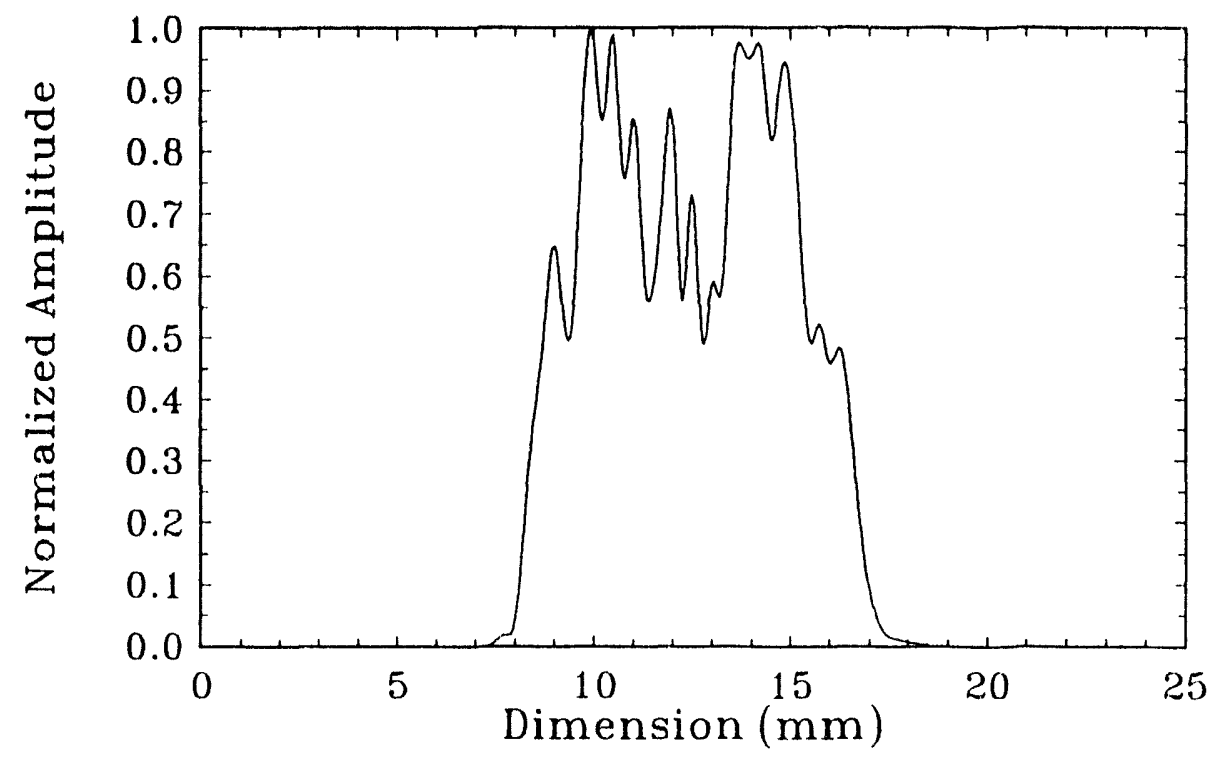

Figure 14: Near-field spatial profile of the $1.06 \mu \mathrm{m}$ pulse produced by a Q-switched, $\mathrm{Nd}$ :YAG laser equipped with a hard edged output coupler.

complete revolution of the target has occurred, approximately 720 laser shots, the feedthru provides linear motion to a new target circumference position. By moving the target in this manner, several thousand new surface shots can be obtained from a single aluminum disk target. A linear/rotary bearing supports one end of the shaft and reduces wobble during target rotation. The focused spot size on target of the $1.06 \mu \mathrm{m}$ beam is monitored with the same reticon/digital sampling scope system that was used for the nearfield measurements of the previous section. As seen in Figure 10, the reticon array is placed behind the final mirror that directs the $1.06 \mu \mathrm{m}$ light into the chamber and monitors the leakage through that mirror. By placing the reticon at the equivalent target distance 


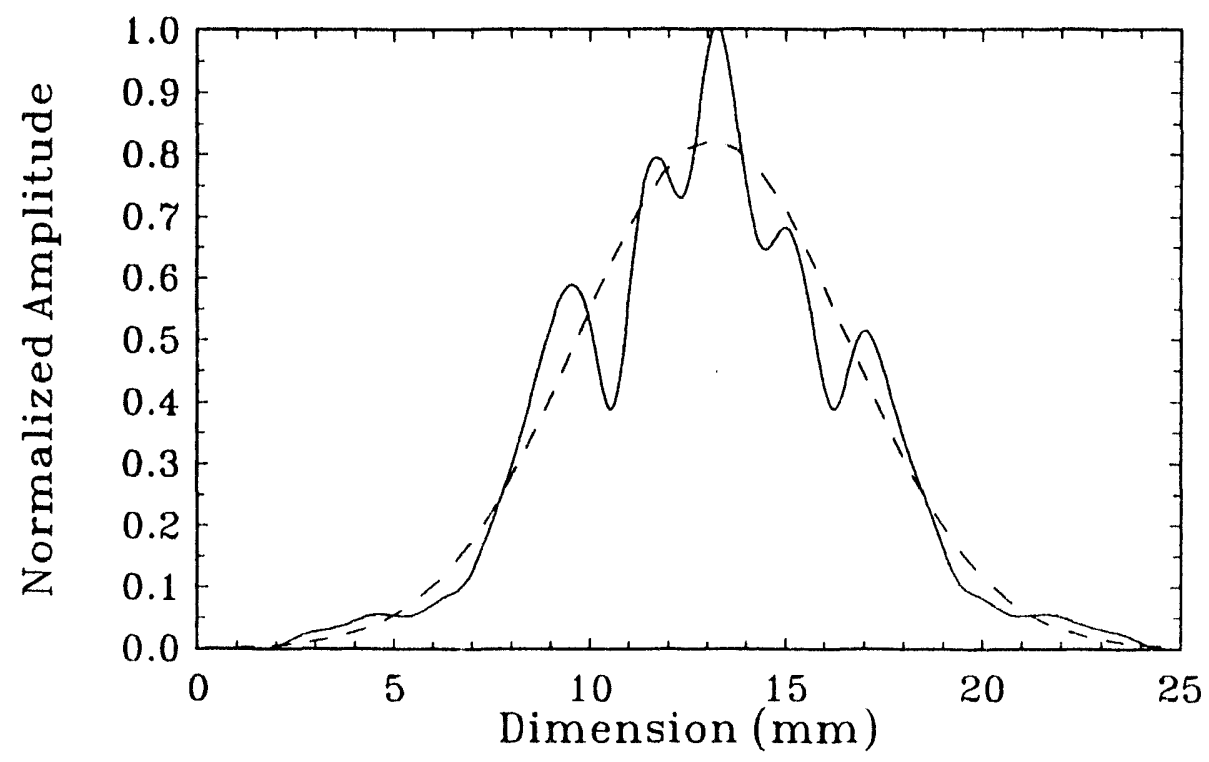

Figure 15: Near-field spatial profile of the $1.06 \mu \mathrm{m}$ pulse produced by a Q-switched, Nd:YAG laser equipped with a variable reflectivity mirror (solid line). The best fit Gaussian (dashed line) is included (FWHM width $8.2 \mathrm{~mm}$ ).

from the mirror, the spot size on target is measured. The spot size measurement is shown in Figure 16, where the circles are the data from the individual pixels of the reticon (25 $\mu \mathrm{m}$ spacing) and the dashed line is the best fit Gaussian through those points. The best fit Gaussian has a FWHM width of $92 \pm 10 \mu \mathrm{m}$ and can be compared to the spot size predicted by focusing the beam profile of Figure 15 (Gaussian FWHM $=8.2 \mathrm{~mm}$ ) through a $500 \mathrm{~mm}$ focal length lens. It can be shown that the relationship between the FWHM spot size at focus ( $\left.\mathrm{d}_{\text {focus }}\right)$ and the FWHM beam diameter (D) of a near Gaussian beam profile at the input of a lens is given by, 


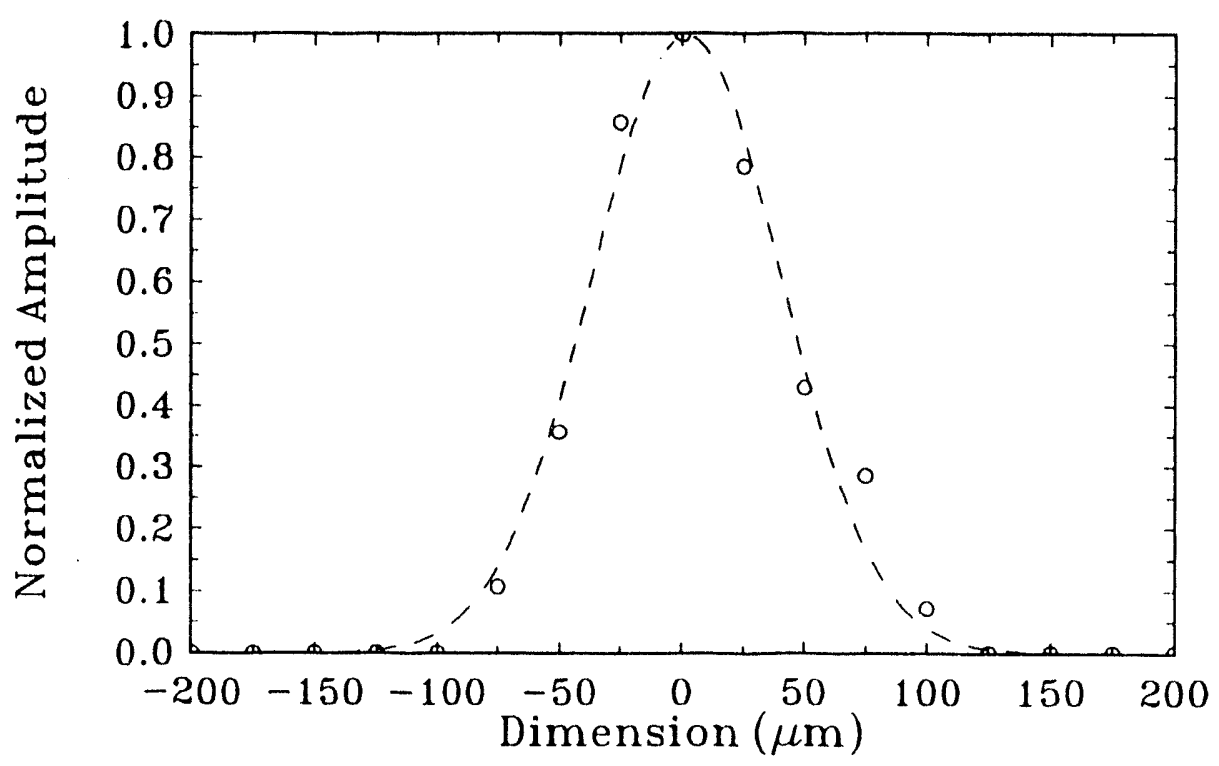

Figure 16: Focused $1.06 \mu \mathrm{m}$ beam at the target surface (circles) with a best fit Gaussian profile (dashed line). The best fit Gaussian to the data has a width (FWHM $=92 \pm 10 \mu \mathrm{m})$.

$$
d_{\text {focus }}=(\text { diff. limit })\left(\frac{0.444 \lambda f}{D}\right)
$$

where $\lambda$ is the wavelength of the light, $f$ is the focal length of the lens, and (diff. limit) $=1$ for a diffraction limited beam. Assuming the beam profile of Figure 15 is diffraction limited, the spot size on target should be $d_{\text {focus }}=28.7 \mu \mathrm{m}$ (from Eq. (47) with $\mathrm{D}=$ $8.2 \mathrm{~mm}, \lambda=1.06 \mu \mathrm{m}, \mathrm{f}=500 \mathrm{~mm}$ ). Since the measured spot size on target is actually $\mathrm{d}_{\text {focus }}=92 \pm 10 \mu \mathrm{m}$, the input beam is $3.2 \pm 0.3$ times diffraction limited. This number agrees with the 3.1 times diffraction limit specification for the laser. A diffraction limited beam is not obtained from the laser due to the diffraction effects present in the beam 
profile as mentioned earlier. The peak intensity on target due to focusing the $200 \mathrm{~mJ}, 9 \mathrm{~ns}$ pulse to a spot size of $92 \mu \mathrm{m}$ is $2 \times 10^{11} \mathrm{~W} / \mathrm{cm}^{2}$. This intensity is deliberately kept low to avoid the production of filamentation, stimulated Brillouin scattering (SBS), and stimulated Raman scattering (SRS) instabilities mentioned in Chapter 1 which could complicate the measurements of the electron density, sound speed, and radial drift in the plasma.

The coordinates assigned to the plasma are shown in Figure 11 which is a close up view of the plasma interaction area. The axial dimension (z) will refer to the direction parallel to the $1.06 \mu \mathrm{m}$ plasma generating beam and $\mathrm{z}=0$ at the target surface. The radial dimension ( $r$ ) is measured in a plane perpendicular to the z-axis and assuming a cylindrically symmetric plasma, the $\mathrm{z}$-axis lies along the axis of the cylinder where $\mathrm{r}=$ 0 . As seen in Figure 11, the diagnostic $266 \mathrm{~nm}$ beam lies in the radial plane.

The ultraviolet diagnostic beam is obtained by frequency doubling the $532 \mathrm{~nm}$ light with an additional KDP rrystal, see Figure 9. This produces a maximum energy of 30 $\mathrm{mJ}$ in a pulse width (FWHM) of $5 \mathrm{~ns}$ at $266 \mathrm{~nm}$ with a pulse-to-pulse energy stability of $\pm 4 \%$. A Pellin-Broca prism is used to separate the $266 \mathrm{~nm}$ from the $532 \mathrm{~nm}$ light and the polarization of the diagnostic $266 \mathrm{~nm}$ beam $\left(\hat{\mathrm{e}}_{\mathrm{pr}}\right)$ is adjusted perpendicular to the radial plane containing $\mathbf{k}_{\mathrm{sc}}$ and $\mathbf{k}_{\mathrm{pr}}$. This geometry will optimize the polarization factor $\mid \hat{\mathrm{k}_{\mathrm{sc}}}$ $\left.x\left(\hat{\mathrm{k}}_{\mathrm{sc}} \times \hat{\mathrm{e}}_{\mathrm{pr}}\right)\right|^{2}$ in Eq. (13) to maximize the Thomson scattering signal. An anti-reflection coated, $300 \mathrm{~mm}$ focal length lens focuses the diagnostic beam into the plasma to a calculated spot diameter (FWHM $=20 \mu \mathrm{m}$ ); The spot size given is calculated by assuming the diagnostic beam has approximately the same diffraction properties as the 
$1.06 \mu \mathrm{m}$ beam from which it is derived. After interacting with the plasma, the diagnostic beam passes out of the other side of the chamber where its energy is monitored with a photodiode connected to a pulse height analyzer. This cilagnostic serves two purposes: it allows for energy normalization of the data, and it provides a measurement of the plasma absorption on the diagnostic beam. Both of these measurements will be discussed in more detail in Chapter 4.

Figure 17 is a ray trace through the collection optics which couple the Thomson scattered light from the plasma into the spectrometer. The Thomson signal is collected at $\theta_{\text {obs }}=45^{\circ}$ with an $f / 12,250 \mathrm{~mm}$ focal length, anti-reflection coated lens. The scattering angle $\theta_{\mathrm{obs}}$ combined with Eq. (11) defines the average wave vector of the

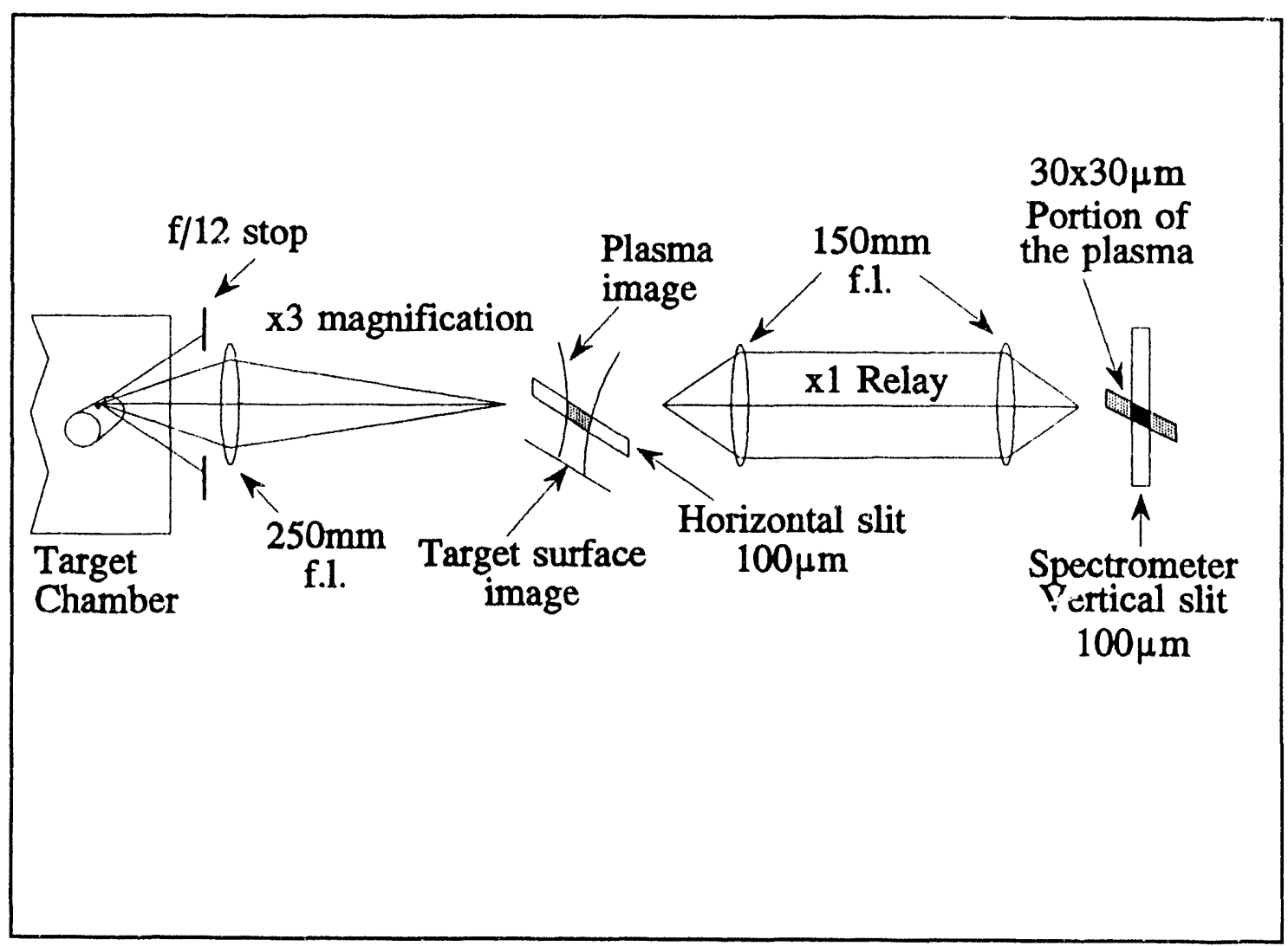

Figure 17: Optical schematic of the collection system which couples the Thomson scattered signal from the plasma into the spectrometer. 
fluctuations that the diagnostic beam scatters from,

$$
k_{f}=\frac{4 \pi}{\lambda_{p r}} \sin \left(\frac{\theta_{o b s}}{2}\right)=180787 \mathrm{~cm}^{-1}
$$

where $\lambda_{\mathrm{pr}}=266 \mathrm{~nm}$. Scattering from ion-acoustic fluctuations causes only a small frequency shift in the scattered light, therefore to derive Eq. (48), the scattered $\left(\lambda_{\text {sc }}\right)$ and probe $\left(\lambda_{\text {pr }}\right)$ wavelengths are assumed equal in Eq. (11). The f-number of the collection lens defines the range of ion-acoustic wave vectors from which the scattered light is collected. In this case, the $\mathrm{f} / 12$ optic collects light scattered from ion-acoustic fluctuations with a maximum wave vector of $\mathrm{k}_{\mathrm{fl}}=189850 \mathrm{~cm}^{-1}$ and a minimum wave vector of $\mathrm{k}_{\mathrm{fl}}$ $=171646 \mathrm{~cm}^{-1}$. This range of wave vectors represents only a $\pm 5 \%$ fluctuation around the average and will not seriously effect the measurements of electron density, sound speed, and radial drift. The sound speed and radial drift measurements could be affected if the wave vector distribution $t \quad$ ge enough to obscure the exact position of the peaks in a collective Thomson scattered signal. Although the range of wave vectors does not affect the measurements of the three plasma quantities considered, the collection of a distribution of wave vectors must be taken into account when performing any type of line width analysis on Thomson spectra. From the $250 \mathrm{~mm}$ focal length lens, the scattered


is further relay imaged, with unity magnification, onto the vertical entrance slit $(100 \mu \mathrm{m}$ wide) of a spectrometer. The cross-slit arrangement selects a $30 \times 30 \mu \mathrm{m}$ portion of the plasma from which scattered light is collected with a depth of observation equal to $\sqrt{2}$ times the diagnostic beam diameter. The spectrometer is a 0.85 meter double 
monochrometer (SPEX Model 1404) with $1200 \mathrm{~g} / \mathrm{mm}$ gratings blazed at $750 \mathrm{~nm}$ and the detection of the $266 \mathrm{~nm}$ scattered light is done in third order. Time-integrated spectral data are acquired through a 1024-element single-intensified reticon array (EG\&G Model 1420UV) mounted at the exit slit of the monochrometer. The spectral resolution achieved by the reticon is $0.032 \AA /$ pixel.

Stray light at the diagnostic wavelength $\lambda_{\mathrm{pr}}$ can limit the detectability of the Thomson scattered signal especially near the target surface where surface scatter can be enormous. This noise source is reduced in two ways, as seen in Figure 9. First, small apertures are placed just before and after the target. These allow only the diagnostic beam to enter the plasma area and reject most of the scattered light generated by the beam passing through the input and output windows of the vacuum chamber. Second, the $1.06 \mu \mathrm{m}$ beam generating the plasma is chopped at $5 \mathrm{~Hz}$ so that odd-numbered spectra contain only background noise at $\lambda_{\mathrm{pr}}$. This background is subtracted from the evennumbered spectra leaving only the Thomson signal. Even with these methods, the accessible region for data acquisition is limited to axial distances $\geq 60 \mu \mathrm{m}$, below which the scatter from the target surface completely saturates the collection system. The background subtraction technique outlined here will be discussed in more detail in Chapter 4.

The intricate timing system needed to coordinate all of the components in this experimental configuration is shown in Figure 18. The timing pulse that synchronizes the system components originates from the Stanford Research Systems, Inc. Model SR540 Chopper which is used to chop the $1.06 \mu \mathrm{m}$ plasma generating beam at $5 \mathrm{~Hz}$. The chopper 


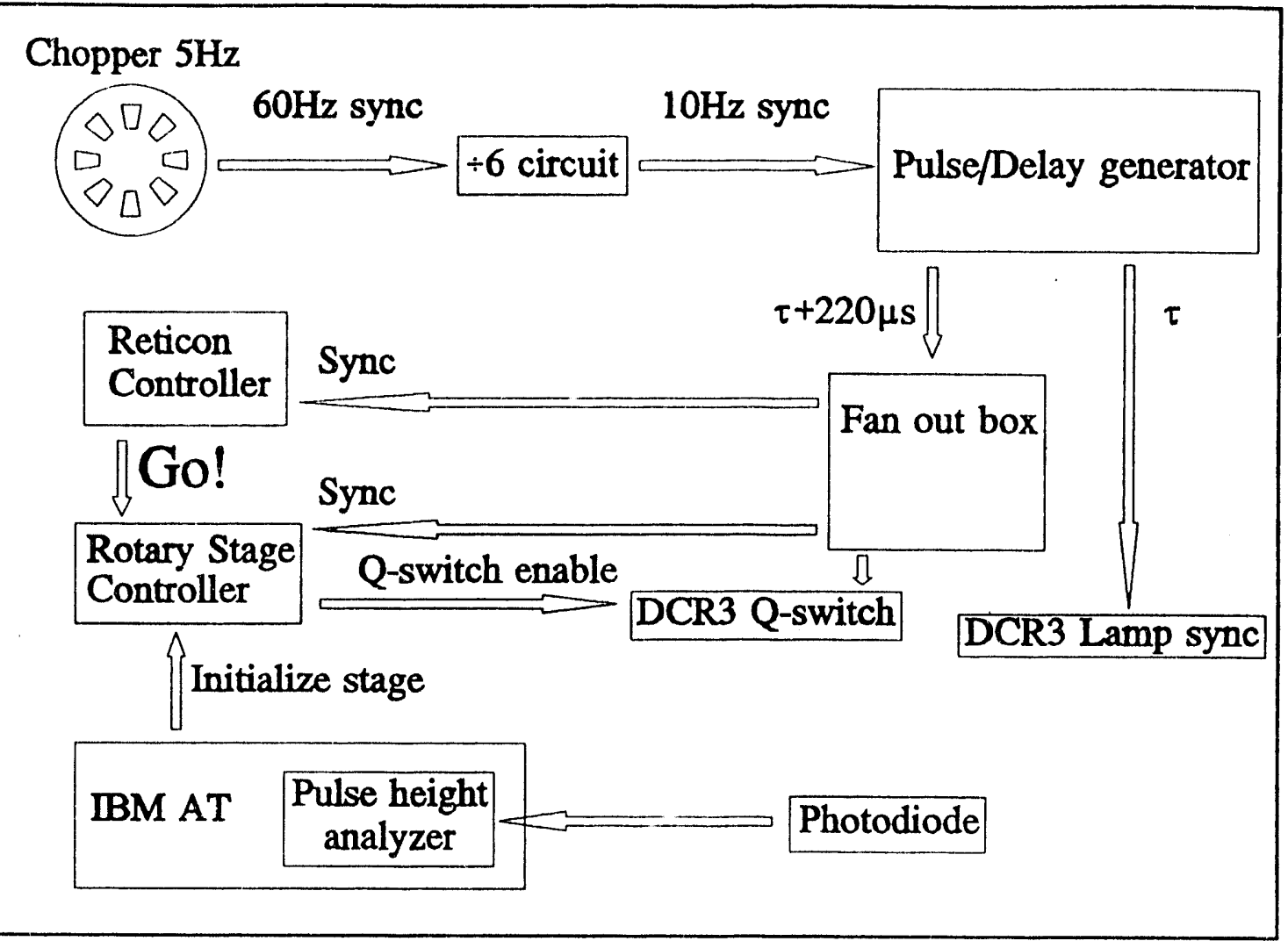

Figure 18: Timing schematic for the Thomson scattering experimental configuration. controller provides a $60 \mathrm{~Hz}$ synchronization signal which is exactly twelve times the chopper frequency. This signal is reduced to a $10 \mathrm{~Hz}$ repetition rate by a divide by 6 circuit and then is used to trigger a Stanford Research Systems, Inc. Model DG535 Pulse/Delay generatur. The pulse/delay generator provides pulses at the $10 \mathrm{~Hz}$ repetition rate to the lamp sync and Q-switch sync inputs of the DCR-3 laser used for the experiment. For optimum pulse energy from the laser, the lamp sync pulses must arrive approximately $220 \mu$ s before the Q-switch sync pulse. Prior to arriving at the laser, the Q-switch sync pulse is sent through a fan out box. The pulses from the fan out box outputs provide exact synchronization between the laser pulse and the EG\&G reticon and AEORTECH rotary stage controller circuits. When an experiment is not being conducted, 
the laser is shuttered by an enable/inhibit pulse from the rotary stage controller which unblocks/blocks the Q-switch pulse from reaching the laser. Without a Q-switch sync, the lamps will continue to fire at $10 \mathrm{~Hz}$ but the oscillator cavity is kept from lasing. With the whole system synchronized at $10 \mathrm{~Hz}$, the steps necessary to collecting data can now be discussed. As mentioned previously, stray light is reduced by alternating Thomson and background shots and then subtracting the background data from the Thomson data which also contains the background. A data point then consists of two laser firings, one in which the chopper is not blocking the plasma generating beam (Thomson shot) and one in which the chopper is blocking the plasma generating beam (background shot). A series of data points can then be added together to provide better signal-to-noise in the collected Thomson signal. The collection of a series of data points begins with initialization of the rotary stage controller by the IBM AT computer. Next, the pulse height analyzer which resides in the IBM AT is started in a free running mode. The analyzer monitors the energy in the diagnostic pulses via a photodiode and will only register data when a pulse is incident on the photodiode. The reticon controller is then started in its data acquisition loop. The loop consists of 20 alternating data acquisitions, the odd data sets are accumulated in one memory location and represent the Thomson signal and the even data sets are accumulated in another memory location and represent the background signal. At the beginning of the data acquisition loop, the controller sends a start signal, indicated by Go! in Figure 18, to the rotary stage controller. The rotary stage controller in turn enables the Q-switch to fire in the laser and simultaneously begins turning the target to a new surface for each laser shot. At the end of the data acquisition sequence, the reticon 
controller sends a stop signal to the rotary stage controller. The rotary controller then proceeds to disable the Q-switch in the laser and stops the target from rotating. At this point, the spectral data from the Thomson and background shots resides in the reticon controller memory and the pulse energies from the diagnostic beam are recorded in the pulse height analyzer. The data reduction techniques used to analyze this raw data will be discussed in detail in Chapter 4.

Two key elements not mentioned to this point have an impact on the experimental operation, rough alignment of the diagnostic beam and target debris. First, a rough alignment of the diagnostic $266 \mathrm{~nm}$ beam with the $1.06 \mu \mathrm{m}$ plasma generating beam is facilitated by the use of the CCD camera (COHU Model 4815-2000) shown in Figure 10. This camera monitors the plasma interaction region of the target surface through a $x 6$ zoom lens coupled with a $\mathrm{x} 3$ telescope which provides an overall magnification of 18 . After generating a plasma from a fresh surface on the target, the diagnostic beam is steered by transverse movement of the $300 \mathrm{~mm}$ f.l. focusing lens, Figure 9 , until it scatters from the crater in the target surface produced from the $1.06 \mu \mathrm{m}$ beam. The scattered light from the crater is monitored by the CCD camera and this rough alignment places the diagnostic beam radially at a distance no further away from the z-axis of the plasma than the FWHM spot size of the $1.06 \mu \mathrm{m}$ heater beam $\approx 100 \mu \mathrm{m}$. The second important element not mentioned to this point that affects the operation of the experiment is target debris. Since the experiment runs at a $10 \mathrm{~Hz}$ repetition rate and a fair amount of energy is present in the plasma generating pulse, $\approx 200 \mathrm{~mJ}$, a large amount of aluminum is stripped from the target surface over a short period of time. The ablated aluminum 
from the target acts not unlike a sputtering source in an optical coater and begins depositing on the windows of the chamber. The windows, if left undisturbed, will eventually become opaque thus blocking the diagnostic and plasma generating beams from entering the chamber as well as inhibiting the scattered light from exiting the chamber. Since the chamber is continuously being evacuated by a turbo pump, a steady flow of helium can be added to the chamber in front of all the windows. The helium is allowed to raise the base pressure of the chamber from its lowest range of $\approx 10^{-7}$ torr to $\approx 10^{-3}$ torr. At room temperature, the helium background has a number density of $\mathrm{n}_{\mathrm{He}}=3 \mathrm{x}$ $10^{14} \mathrm{~cm}^{-3}$ and represents an insignificant fraction of the number densities of aluminum generated in the laser plasma which are greater than $10^{18} \mathrm{~cm}^{-3}$. Since the concentration of helium is small, it will not affect the plasma generation. On the other hand, the helium background does affect the mean free path of aluminum once it disperses from the plasma generating area. The mean free path, $1_{\mathrm{Al}}$, of an aluminum atom in a binary mixture of " helium and aluminum is given by, ${ }^{132}$

$$
l_{A l}=\frac{4}{4 \pi \sigma_{A l}^{2} n_{A l}+\pi\left(\sigma_{A l}+\sigma_{H e}\right)^{2} n_{H e}}
$$

where $\sigma$ is the diameter of the particle and $n$ is the number density. Assuming the helium has much higher number density than the aluminum far from the plasma source and the diameter of aluminum and helium atoms is the same, $\approx 3$ Angstroms, $\mathrm{n}_{\mathrm{Al}}$ can be set equal to zero in Eq. (49) and $\sigma_{\mathrm{Al}}=\sigma_{\mathrm{He}}=3$ Angstroms. The mean free path for aluminum with the background density of helium at $\mathrm{n}_{\mathrm{He}}=3 \times 10^{14} \mathrm{~cm}^{-3}$ from Eq. (49) is then $\mathrm{l}_{\mathrm{Al}}=1.2 \mathrm{~cm}$. Since the distance from the plasma to the windows of the chamber 
is $30 \mathrm{~cm}$, the aluminum atoms will suffer approximately 25 collisions before reaching the windows. In spite of helium presence, the windows of the chamber did have to be replaced a couple of times over the duration of the experiment.

This chapter has described in detail the collective Thomson scattering experimental configuration used for obtaining two-dimensional electron density, sound speed, and radial drift profiles from a collisional laser plasma. Some effort has been made to assemble a system which will repetitively generate a plasma which is reproducible in the three quantities measured. The reproducibility allows a two-dimensional image of the plasma to be generated from a collection of single point measurements from different plasma shots. The high repetition rate at which the plasma is formed allows noise reduction techniques to be used on the data. Other considerations such as stray light reduction, system timing, rough diagnostic beam alignment, and target debris have also been discussed. The actual degree of reproducibility of the three quantities, electron density, sound speed, and radial drift in the plasma will be analyzed in the next chapter. 
Chapter IV: Data acquisition and analysis

In this chapter, collective Thomson scattering from ion-acoustic waves will be used as a diagnostic to obtain two-dimensional electron density, sound speed, and radial drift profiles of a collisional laser plasma. The data presented in this chapter represents the first time detailed measurements of a collisional, laser-generated plasma have been made using $266 \mathrm{~nm}$ collective Thomson scattering. The Thomson scattering measurements will be conducted on a point to point basis, and data from different plasma shots will be collected together to form the overall two-dimensional image of the three plasma quantities listed above. The plasma generated for these experiments must be reproducible in order for the data to be meaningful. The techniques used to generate a reproducible plasma have been outlined in Chapter 3, and this chapter will demonstrate that the plasma is in fact reproducible in electron density, sound speed, and radial drift. Along with plasma reproducibility, the diagnostic beam and the field of view of the collection optics must be aligned absolutely so that the exact position is known in the plasma for each data point. The techniques for positioning the diagnostic system absolutely at a given point are different for the axial and radial directions in the plasma and will be discussed. After the exact position in the plasma has been established, the process of data collection and analysis can begin. The collection of Thomson spectra and the subsequent analysis needed to measure the sound speed and radial drift is simple and straight forward, but in order to make the electron density measurements, relative energy calibration of the Thomson scattering collection system and a much more involved analysis are required. The process 
for energy calibrating the system using Rayleigh scattering will be discussed along with the additional data analysis necessary for accurate electron density measurements from collective Thomson scattering. The problems of background light from the plasma, inverse bremsstrahlung and refractive turning of the diagnostic beam mentioned in Chapter 1 will be considered as they affect the acquisition of the Thomson scattered signal. The two-dimensional data obtained from the collisional plasma in these experiments will be compared to the same quantities predicted by a LASNEX simulation for the plasma. Detailed comparisons between experiments and computer simulations of this type are important for benchmarking the simulations for future calculations. In addition to LASNEX, several simple but physically based models will be presented to demonstrate the self-consistency of the electron density, sound speed, and radial drift measurements. The data from these experiments will also show inconsistent peak height asymmetries which to first order cannot be accounted for by traditional heat transport alone. New developments in Thomson scattering theory are needed to investigate the peak height asymmetries and their relation to heat transport. These new developments will be presented in the next chapter.

As outlined in Chapter 3, collective Thomson spectra are accumulated by averaging two sets of ten shots for each point in the plasma. One set of ten shots is the Thomson signal plus the background noise and the other set is just the background noise. The accumulation of data increases the signal-to-noise ratio over that of single-shot acquisition by approximately the square root of the number of shots accumulated. While at high densities, signal-to-noise is not an issue, averaging allows for measurements at 


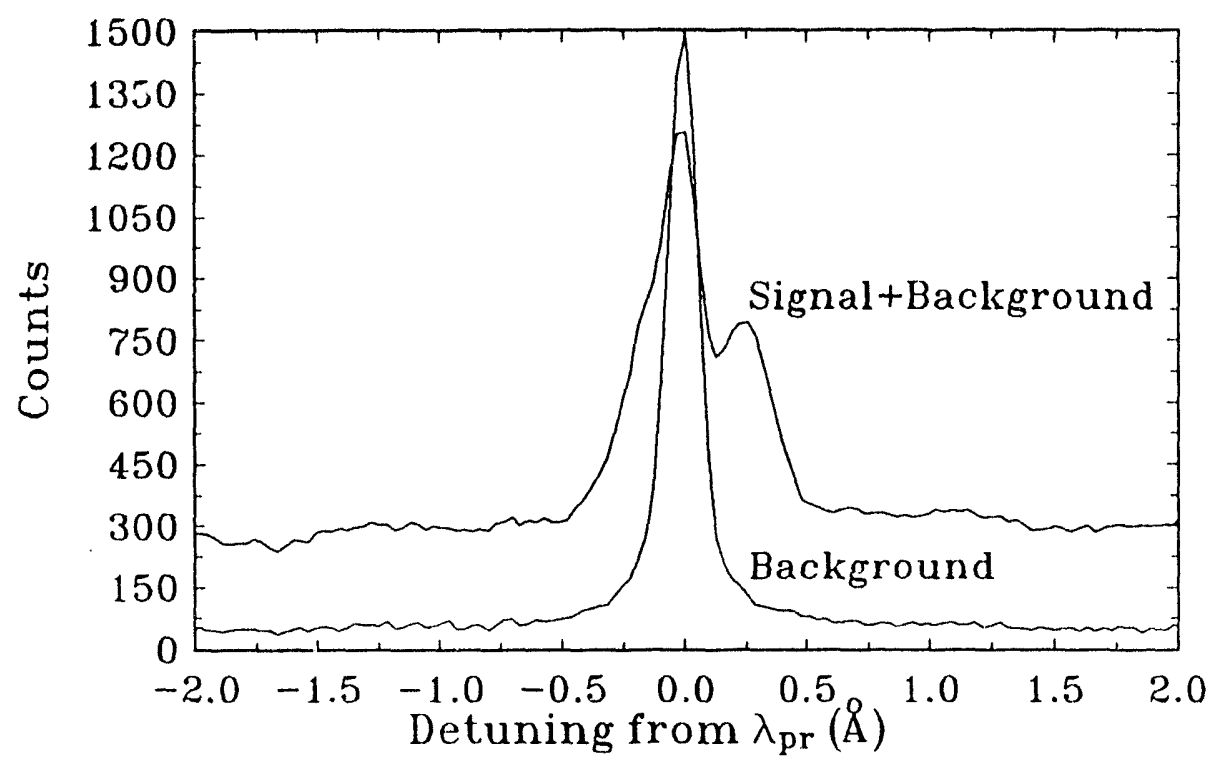

Figure 19: Raw data of collective Thomson scattering from a single point in the plasma which entails signal + background and background data sets.

much lower densities than single-shot operation. Figure 19 shows actual Thomson scattered data collected from one point in the aluminum plasma. The background signal is due to scattered light from the target surface that enters the collection optics and lies at the laser frequency which is at 0 on the $x$-axis of the graph. As seen in Figure 19, the Thomson plus background signal not only contains background scatter from the diagnostic beam but also a baseline level that is due to bremsstrahlung emission from the plasma. The bremsstrahlung background is not entirely flat and the noise associated with the background affects the lowest Thomson signal level that is detectable. Although in gen ral, background noise from plasma emission also contains resonant lines from bound- 


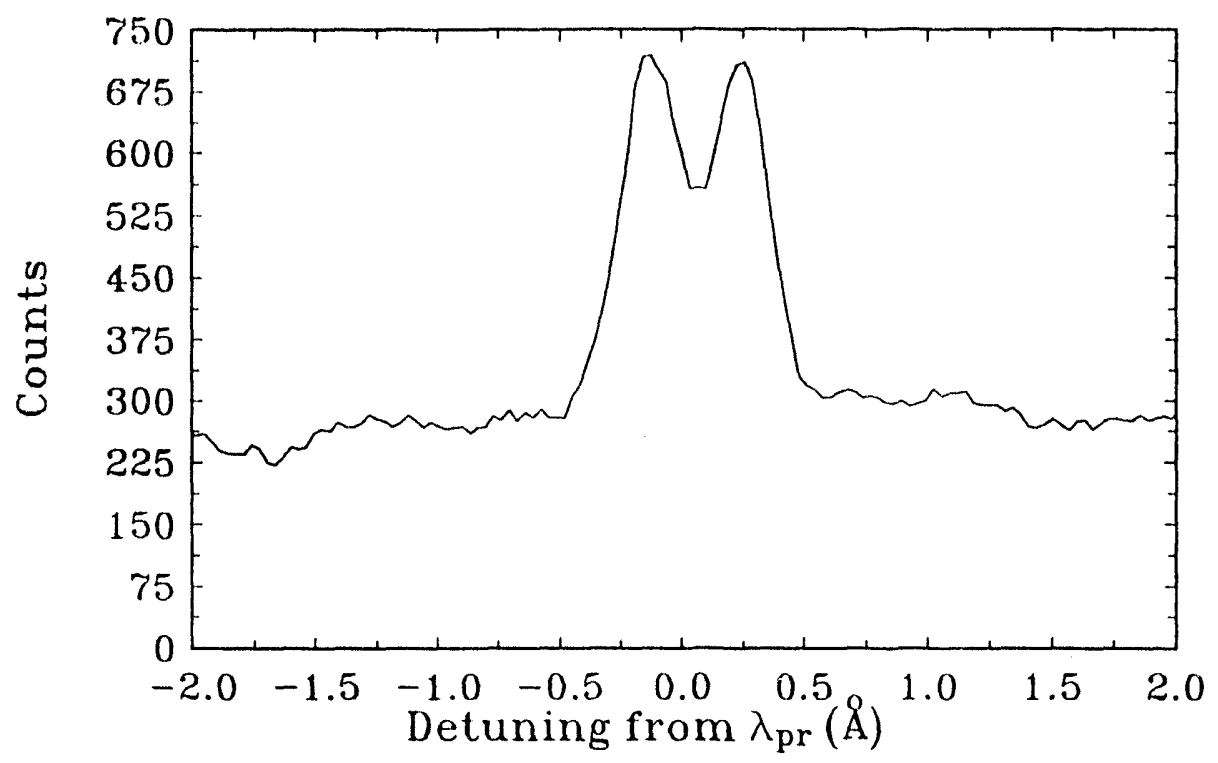

Figure 20: Collective Thomson scattered signal obtained after background subtraction. bound transitions which can interfere with the signal, the aluminum plasma has no such lines in the portion of the spectrum around $266 \mathrm{~nm}$. The reason for collecting the background target surface scatter separately from the Thomson plus background signal is evident by Figure 19. The collective Thomson signal should have two peaks one of which is obscured by the target surface scatter in the raw data. The target surface scatter can be eliminated by subtracting a fraction of the background signal from the Thomson plus background signal. The resulting spectra is shown in Figure 20. The two peaks in the collective Thomson signal are now both visible with the bremsstrahlung baseline still present. Since the amount of scatter from the target surface is influenced by the presence of the plasma, the target surface scattering level in the Thomson plus background signal 
is different than the level collected in just the background signal with no plasma present. This is the reason why only a fraction of the background signal need be subtracted to eliminate the target scatter from the collective Thomson spectrum. This fraction typically varies from $40-100 \%$ depending on the plasma shot and the exact amount subtracted directly affects the valley depth between the peaks in the Thomson spectra. Since the electron density is related to the total number of counts under the Thomson peaks, the subjective background subtraction introduces some human error into the electron density measurements. Fortunately the valley depth has a reasonable range of locations: low enough to eliminate the target scattered peak from the Thomson spectrum but no lower than the baseline of the Thomson spectrum. Since the valley represents a narrow section of the Thomson spectrum in these experiments, the reasonable range of valley depths does not significantly effect the number of counts under the Thomson peaks or the electron density measurements. Unfortunately even with background subtraction, the scatter from the target surface becomes too large at axial distances $\leq 60 \mu \mathrm{m}$ to back out a discernable Thomson scattered signal. It should also be noted that at axial distances $\geq 100 \mu \mathrm{m}$, background subtraction is not typically needed since the target surface scatter at these axial distances is much smaller than the Thomson signal.

Of the three quantities, electron density, sound speed, and radial drift, the measurement of electron density from collective Thomson scattering is the most involved part of the data acquisition. The electron density is measured by utilizing Eq. (13) which relates the electron density to the Thomson scattered power. The time integral of the scattered power, the scattered energy, is monitored by the reticon detector on the back 
end of the collection system used in these experiments, see Chapter 3. The reticon detector records the time integrated ion-acoustic Thomson spectrum and the sum of the counts under the ion-acoustic spectrum can be related to the total scattered energy when the collection system is properly calibrated. The total scattered energy collected $\left(E_{s c}{ }^{T}\right)$ that comprises the ion-acoustic part of the Thomson spectrum can be found by integrating Eq. (13) over low frequencies and over all time,

$$
E_{s c}^{T}\left(\vec{k}_{s c}\right) d \Omega=\int_{s \text { mall } \omega_{\alpha c}}\left[\int_{-\infty}^{\infty} P_{s c}\left(\vec{k}_{s c}, \omega_{s c}\right) d t\right] d \omega_{s c} d \Omega=E_{p r} e^{-\Delta} \sigma_{T} n_{c}\left(\frac{V}{A}\right) \frac{S_{T}^{i}\left(\vec{k}_{i a}\right)}{2 \pi} \beta_{a d j} d \Omega
$$

where $\mathrm{E}_{\mathrm{pr}}$ is the energy in the diagnostic pulse, the polarization factor $\mid \mathrm{k}_{\mathrm{sc}}^{\wedge} \times\left(\mathrm{k}_{\mathrm{sc}} \mathrm{x}^{-}\right.$ $\left.\hat{\mathrm{e}}_{\mathrm{pr}}\right) \mid$ has been set equal to 1 because $\hat{\mathrm{e}}_{\mathrm{pr}} \perp \hat{\mathrm{k}}_{\mathrm{sc}}, \mathrm{k}_{\mathrm{ia}}$ is the ion-acoustic or fluctuation wave vector from Eq. (48), and the provision for a time-dependent electron density $\left[\mathrm{n}_{\mathrm{e}}{ }^{*}(\mathrm{t})\right]$ and total ion cross section $\left[\mathrm{S}_{\mathrm{T}}{ }^{\mathrm{i}}\left(\mathrm{k}_{\mathrm{ia}}, \mathrm{t}\right)\right]$ is included through $B_{\mathrm{adj}}$. The quantities $n_{e}$ and $S_{T}{ }^{i}\left(k_{i a}\right)$ represent the maximum values that $\left[n_{e}^{*}(t)\right]$ and $\left[S_{T}{ }^{i *}\left(k_{i a}, t\right)\right]$ obtain, respectively, and $B_{\mathrm{adj}} \leq 1$ contains the temporal integration, i.e.,

$$
\beta_{a d j}=\frac{\int_{-\infty}^{\infty} P_{p r}(t) n_{e}^{*}(t) S_{T}^{i *}\left(k_{i a}, t\right) d t}{E_{p r} n_{e} S_{T}^{i}\left(k_{i a}\right)}
$$

If $n_{e}{ }^{*}(t)$ and $S_{T}{ }^{i *}\left(k_{i a}, t\right)$ are stationary over the width of the diagnostic pulse, $\beta_{\text {adj }} \approx 1$. The $\beta_{\text {adj }}$ parameter is necessary for analysis of the data for these experiments because of the length of the diagnostic pulse compared to the length of the plasma generating pulse. The $266 \mathrm{~nm}$ diagnostic pulse length is $5 \mathrm{~ns}$ (FWHM) and the pulse length of the $1.06 \mu \mathrm{m}$ beam generating the plasma is 9ns (FWHM). Therefore with the peak of the diagnostic 




Figure 21: Time dependent electron density at $\mathrm{r}=0$ and $\mathrm{z}=65 \mu \mathrm{m}$ as computed by LASNEX. The 5ns (FWHM) Gaussian diagnostic pulse is included for reference.

and plasma generating pulses overlapped in time, a fraction of the time development of the plasma is probed around the peak of the plasma generating pulse, and the plasma quantities being measured can by no means be considered constant over the 5 ns diagnostic pulse length. The only way to approximate the value of $B_{\mathrm{adj}}$ for the laser plasma being studied is to assume the temporal development of the plasma quantities: electron density $\left(\mathrm{n}_{\mathrm{e}}^{*}(\mathrm{t})\right)$, electron temperature $\left(\mathrm{T}_{\mathrm{e}}^{*}(\mathrm{t})\right)$, and average ionization state $\left(\mathrm{Z}^{*}(\mathrm{t})\right)$ predicted by LASNEX are generally correct. Figure 21, Figure 22, and Figure 23 show the temporal development of the electron density, electron temperature, and average ionization state predicted by LASNEX as a function of time at axial distance $z=65 \mu \mathrm{m}$ and radial 


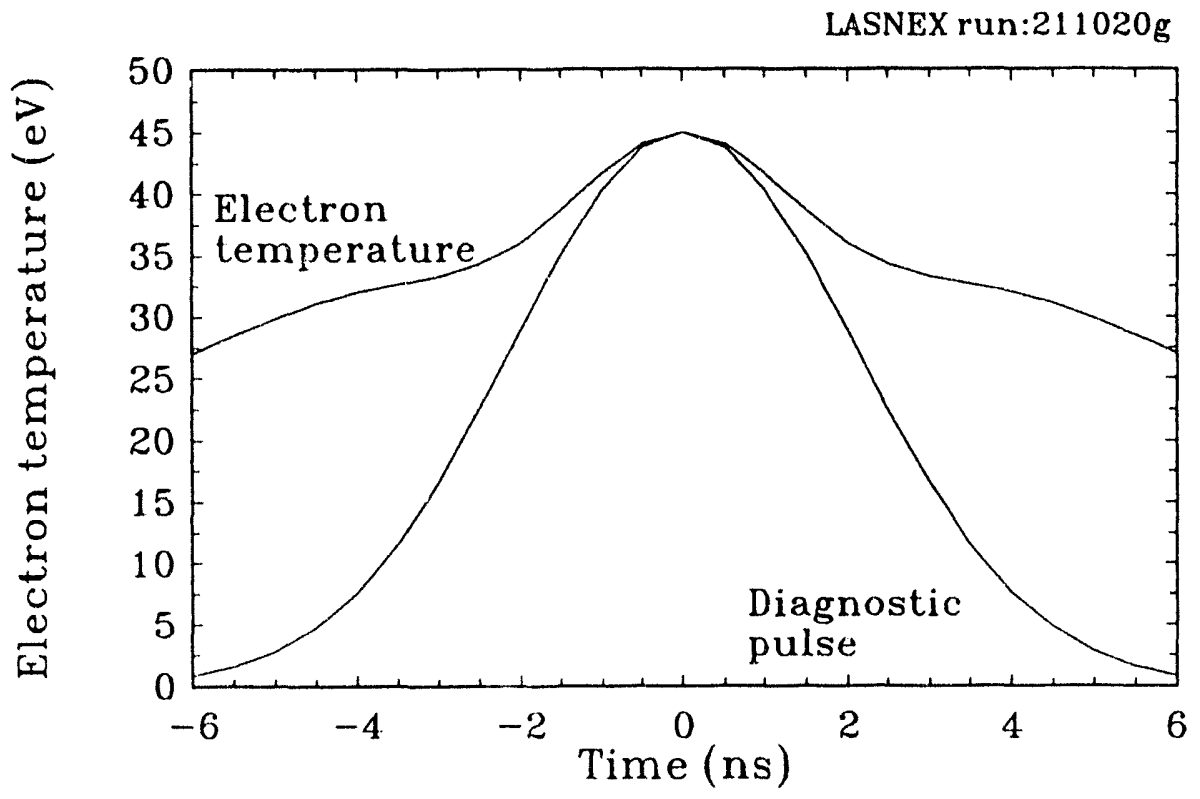

Figure 22: Time dependent electron temperature at $r=0$ and $z=65 \mu \mathrm{m}$ as computed by LASNEX. The 5ns (FWHM) Gaussian diagnostic pulse is included for reference.

distance $\mathrm{r}=0 \mu \mathrm{m}$. Since the LASNEX calculation is only carried out for times less than 0ns, the temporal development of the quantities for times greater than 0 in the figures have been approximated to be syrnmetric with the values at times less than 0 . The peak of the diagnostic and plasma generating pulses lie at time $=0 \mathrm{~ns}$, and the 5ns (FWHM) Gaussian diagnostic pulse, $P_{p r}(t)=\exp \left(-(t / 3)^{2}\right) / 3 \sqrt{\pi}$, is shown in the figures for reference. As shown by Figure 21, Figure 22, and Figure 23, the plasma quantities are not quite constant over the duration of the diagnostic pulse. The time dependent electron

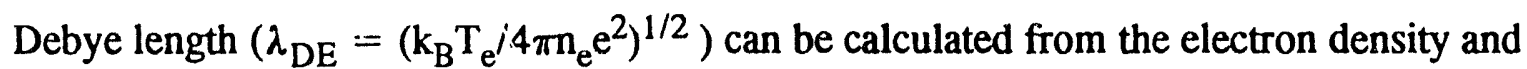
temperature data which in turn can be used to calculate the time dependent $\alpha^{*}(\mathrm{t})$ with 
LASNEX run:211020g



Figure 23: Time dependent average ionization state at $\mathrm{r}=0$ and $\mathrm{z}=65 \mu \mathrm{m}$ as computed by LASNEX. The 5ns (FWHM) Gaussian diagnostic pulse is included for reference.

$\alpha^{*}(\mathrm{t})=1 / \mathrm{k}_{\mathrm{ia}} \lambda_{\mathrm{DE}}(\mathrm{t})$. The time dependent total ion cross section $\mathrm{S}_{\mathrm{T}}{ }^{\mathrm{i}}\left(\mathrm{k}_{\mathrm{ia}}, \mathrm{t}\right)$ is then found from Figure 8 using the $\alpha^{*}(\mathrm{t})$ and $Z^{*}(\mathrm{t})$ data under the assumption that the electron and ion temperatures in this collisional aluminum plasma are about the same, $\mathrm{ZT}_{\mathrm{e}} / \mathrm{T}_{\mathrm{i}} \approx \mathrm{Z}$. Since the plasma is collisional and the electron-ion energy equilibration time is less than the plasma generating beam pulse length, a result that will be calculated from the experimental data, the approximation that the electron and ion temperatures are about the same is justified. With $n_{e}{ }^{*}(t), S_{T}{ }^{i *}\left(k_{i a}, t\right)$, and $P_{p r}(t), B_{a d j}$ can be calculated from Eq. (51). Figure 24 displays the results for $\beta_{a d j}$ at radial position $r=0$ and axial positions between $65 \mu \mathrm{m}$ and $235 \mu \mathrm{m}$ as determined by LASNEX. The average $\beta_{\text {adj }}$ over the range 


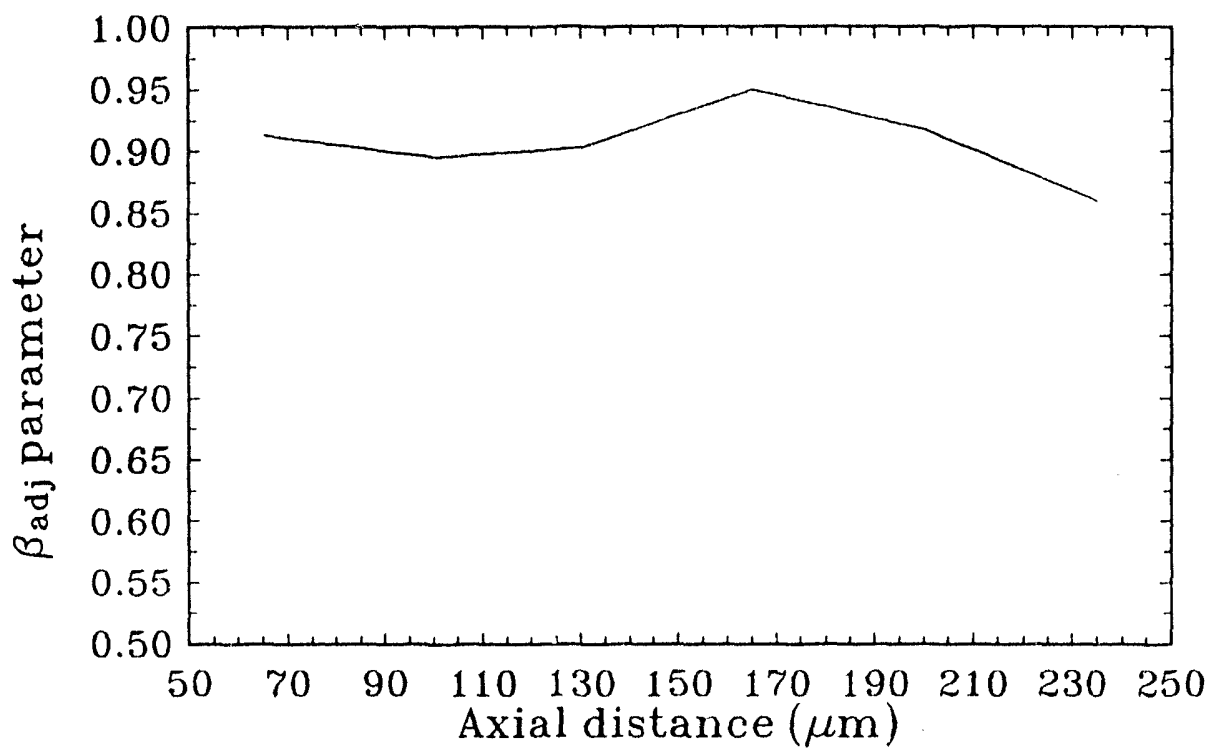

Figure 24: The parameter $B_{a d j}$ at $r=0$ as a function of the axial distance $z$.

of axial positions is $\approx .9$ and these calculations show that the Thomson scattering efficiency into the ion-acoustic feature will be reduced by $10 \%$ from the efficiency that would be obtained if the quantities $n_{e}{ }^{*}(t)$ and $S_{T}{ }^{i *}\left(k_{i a}, t\right)$ where constant in time. The effects of non-stationary plasma conditions on the Thomson scattering efficiency could be eliminated entirely if a shorter $(\approx 1 \mathrm{~ns})$ diagnostic pulse where used, but unfortunately this pulse duration cannot be achieved with the current experimental configuration.

The values of $B_{a d j}, S_{T}{ }^{i}\left(k_{i a}\right)$, and $e^{-\Delta}$ are needed in Eq. (50) to determine the electron density from the Thomson scattered power. As in the previous section, $S_{T}{ }_{T}{ }\left(\mathbf{k}_{\mathrm{ia}}\right)$ can be determined from Figure 8 with knowledge of $\alpha$ and the average ionization state $\mathrm{Z}$ again with the assumption that the electron and ion temperatures are about equal. As 
will be shown later, the sound speed data from the experiment indicates that the range of values for $\alpha$ and $Z$ in the region of interest in the plasma is $3 \leq \alpha \leq 15$ and $5 \leq Z$ $\leq 8$, yielding $S_{\mathrm{T}}^{i}\left(k_{\mathrm{ia}}\right)=0.80 \pm 0.06$ from Figure 8. The factor $\mathrm{e}^{-\Delta}$ is a measure of how much of the diagnostic beam is absorbed by the plasma through inverse bremsstrahlung. The absorption is monitored by the photodiode/pulse height analyzer combination described in Chapter 3. In performing the experiments, there is no detectable absorption due to the plasma in the accessible region (axial distances $>60 \mu \mathrm{m}$ ) in excess of the inherent $\pm 4 \%$ amplitude noise on the $266 \mathrm{~nm}$ diagnostic beam. Therefore, the absorption factor is given by $\mathrm{e}^{-\Delta}=0.96 \pm 0.04$.

As mentioned earlier, the number of counts monitored by the reticon array can be related to the Thomson scattered energy $\mathrm{E}_{\mathrm{sc}}{ }^{\mathrm{T}}$ with a properly calibrated collection system. This calibration is obtained through Rayleigh scattering. Once the system has been aligned to maximize the Thomson signal, the plasma is turned off by blocking the $1.06 \mu \mathrm{m}$ generating beam. The chamber is then filled with a gas and Rayleigh scattering is performed. The gas passes through a $5 \mu \mathrm{m}$ filter before entering the chamber. This removes the dust particles that can scatter large amounts of light, especially in the forward direction, thereby producing spurious results ${ }^{133}$. In the experimental geometry used for these experiments, the Rayleigh scattered energy $\left(E_{s c}{ }^{R}\right)$ is given by a formula similar to Eq. (50) for the Thomson scattered energy $\left(E_{s c}{ }^{T}\right)$,

$$
E_{s c}^{R} d \Omega=E_{p r}\left(\frac{16 \pi^{4} \gamma_{m o l}^{2}}{\lambda^{4}}\right)\left(\frac{P}{k_{B} T}\right)\left(\frac{V}{A}\right) d \Omega
$$

where $\gamma_{\mathrm{mol}}$ is the molecular polarizability of the gas, $\lambda$ is the wavelength of the 


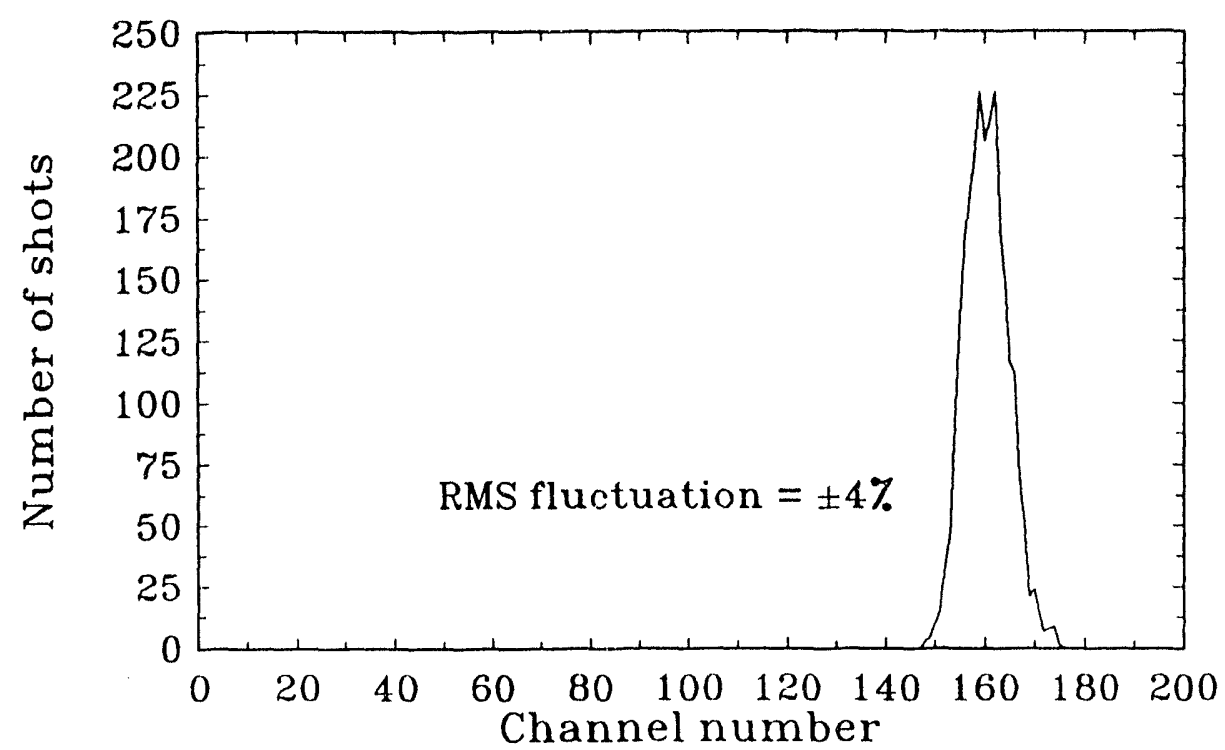

Figure 25: Pulse height analyzer output of $266 \mathrm{~nm}$ diagnostic beam energy from the Rayleigh calibration. The RMS pulse-to-pulse amplitude fluctuation of the diagnostic beam energy is $\pm 4 \%$.

diagnostic beam, $\mathrm{k}_{\mathrm{B}}$ is the Boltzmann constant, and $\mathrm{P}$ and $\mathrm{T}$ are the pressure and temperature of the gas, respectively. Taking the ratio of Eq. (50) to Eq. (52) and rearranging, the electron density becomes,

$$
n_{e}=\left(\frac{16 \pi^{4} \gamma_{m o l}^{2}}{\lambda^{4} \sigma_{T} S_{T}^{i}\left(k_{i a}\right)}\right)\left(\frac{P}{k_{B} T}\right)\left(\frac{E_{s c}^{T} e^{\Delta}}{E_{s c}^{R} \beta_{a d j}}\right)
$$

The Thomson and Rayleigh scattered signals can be normalized (i.e. $\mathrm{E}_{\mathrm{sc}} / \mathrm{E}_{\mathrm{pr}} \rightarrow\left\langle\mathrm{E}_{\mathrm{sc}}\right\rangle$ ) by monitoring the diagnostic power $\mathrm{E}_{\mathrm{pr}}$ with the photodiode/pulse height analyzer combination. This normalization provides a relative calibration between the counts 


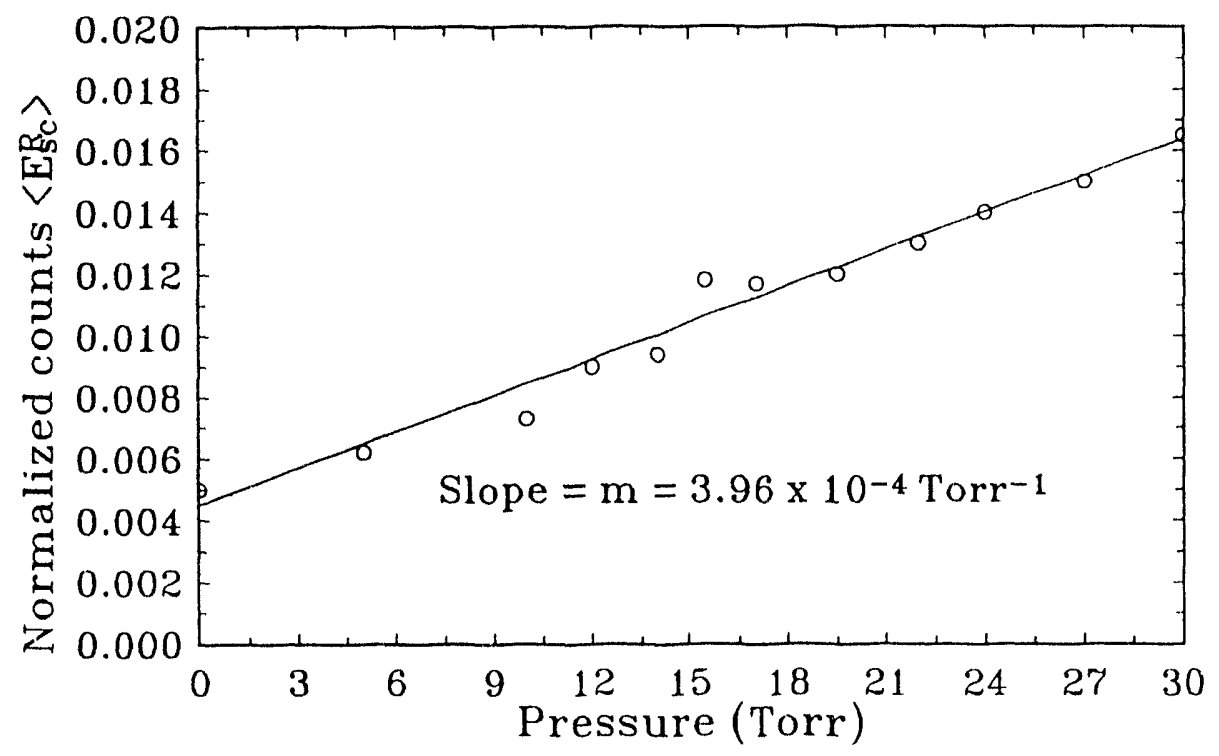

Figure 26: Calibration curve for the normalized counts $\left\langle\mathrm{E}_{\mathrm{sc}}{ }^{\mathrm{R}}\right\rangle$ from Rayleigh scattering in oxygen. The best fit (line) to the data (circles) has a slope of $\mathrm{m}=3.96$ x $10^{-4}$ Torr $^{-1}$.

recorded by the reticon array and the scattered energy. The absolute calibration of the collection system involves performing Rayleigh scattering at several different gas pressures. Extensive signal averaging is used to reduce the effects of noise sources such as dust particles. At each point, the scatter from 2500 laser shots is integrated by the reticon array and normalized to the recorded diagnostic energy on the photodiode/pulse height analyzer. The output from the pulse height analyzer from one of the data points is shown in Figure 25. The noise on the diagnostic beam is found from the RMS fluctuation of the signal and as stated before is $\pm 4 \%$. The normalized signal collected by the reticon array is plotted versus gas pressure and the linearity from Eq. (52) is 
confirmed and shown in Figure 26 for scattering from oxygen $\left(\mathrm{O}_{2}\right)$ gas at room temperature $T=293 \mathrm{~K}$. Deviations in the linearity at higher gas pressures are indicative of gas breakdown and the pressure must be kept below this breakdown threshold in order to produce an accurate calibration. An appropriate choice of gas must be made on the basis of the diagnostic wavelength. The intensities at which the Rayleigh scattering is performed are typically $>10^{10} \mathrm{~W} / \mathrm{cm}^{2}$, therefore, one- and two-photon resonant transitions in the gas at the probe wavelength should be avoided. Such resonances can unpredictably change the polarizability of the gas. For example, the Lyman-BirgeHopfield lines ${ }^{134}$ of $\mathrm{N}_{2}$ are two-photon allowed in the vicinity of $266 \mathrm{~nm}$. On this basis, $\mathrm{O}_{2}$ is chosen for calibration and its molecular polarizability is $\gamma_{\mathrm{mol}}=1.76 \times 10^{-24}$ $\mathrm{cm}^{3}$ at the diagnostic wavelength $\lambda=266 \mathrm{~nm}^{135}$. From the Rayleigh scattering results in Figure 26, the normalized Rayleigh scattered energy is related to the pressure of the gas $P$ by a slope $m=3.96 \times 10^{-4} \operatorname{Torr}^{-1}\left(<E_{s c}{ }^{R}>=m P\right)$. This relation with the normalized Thomson scattered energy $\left\langle\mathrm{E}_{\mathrm{sc}}{ }^{\mathrm{T}}>\right.$ can be substituted into Eq. (53) to yield,

$$
\left.n_{e}=\left(\frac{16 \pi^{4} \gamma_{m o l}^{2}}{\lambda^{4} \sigma_{\gamma} S_{T}^{i}\left(k_{i a}\right)}\right)\left(\frac{\left\langle E_{s c}^{T}>e^{\Delta}\right.}{m k_{B} T \beta_{a d j}}\right)=1.46 \pm 0.29 \times 10^{19}<E_{s c}^{T}\right\rangle \mathrm{cm}^{-3}
$$

where the final equality is found by substitution of the values for the quantities given earlier and the error associated with the electron density measurements is $\pm 20 \%$.

The sound speed and radial drift measurements of the plasma are easier to obtain than the electron density measurements because they are evaluated from the peak positions in the collective Thomson spectra. The sound speed $c_{\mathrm{s}}$ is related to the peak separation $\Delta \omega_{\text {peak }}$ in the collective Thomson scattered signal through the dispersion relation for ion- 
acoustic waves,

$$
\Delta \omega_{\text {peak }}=\frac{2 \pi c \Delta \lambda_{\text {peak }}}{\lambda^{2}}=2 \omega_{i a}=2 k_{b a} c_{s}
$$

where $\lambda=266 \mathrm{~nm}$ is the diagnostic wavelength, $\mathrm{c}$ is the speed of light, and $\mathrm{k}_{\mathrm{ia}}=180787$ $\mathrm{cm}^{-1}$ is the ion-acoustic wave vector. Since the resolution at the reticon detector is 0.032 angstroms/pixel, Eq. (55) can be rewritten for the sound speed in terms of the separation of the peaks in pixels on the reticon array,

$$
c_{s}=2.36 \times 10^{5} \Delta \lambda_{\text {pixels }} \quad \frac{\mathrm{cm}}{\mathrm{s}}
$$

The sound speed is given by Eq. (45) for this collisional plasma, the average ionization state/electron temperature product $\left((\mathrm{Z}+5 / 3) \mathrm{T}_{\mathrm{e}}\right)$, which is basically the sound speed squared, can also be written in terms of the peak separation in pixels. Since $3 \leq \alpha \leq$ 15 for this experiment, the $\alpha^{2} /\left(\alpha^{2}+1\right)$ ratio in Eq. (45) can been set equal to 1 . With the mass of the aluminum ion given as $\mathrm{m}_{\mathrm{i}}=4.51 \times 10^{-23} \mathrm{~g}$ and the electron and ion temperatures assumed equal, Eq. (56) and Eq. (45) are combined to yield the $(\mathrm{Z}+5 / 3) \mathrm{T}_{\mathrm{e}}$ product,

$$
\left(Z+\frac{5}{3}\right) T_{e}=1.58 \Delta \lambda_{\text {pixels }}^{2} e V
$$

The sound speed measurements from the experiment will be displayed in terms of the $(Z+5 / 3) T_{e}$ product from this equation instead of just $c_{s}$ from Eq. (56). The error associated with the measurements is given by a plus or minus pixel error reading of the peak separation on the reticon array, $\Delta \lambda_{\text {pixel }}= \pm 1$. The radial drift velocity is measured 
by the Doppler shift in the collective Thomson spectrum. The shift is measured by finding the position exactly halfway between the peaks in the spectrum and comparing that position to the position of the diagnostic wavelength $\lambda_{\mathrm{pr}}$ on the reticon array. The shift $\Delta \lambda_{\text {shift }}$ from $\lambda_{\mathrm{pr}}$ can be related to the radial drift velocity $U_{\text {rad }}$. Since the Thomson scattering process takes place solely in the radial plane, see Chapter 3 , the ion-acoustic wave vector $\mathbf{k}_{\mathrm{ja}}$ lies in the radial plane, see Eq. (11), and the frequency shift term $\left(\mathbf{k}_{\mathrm{fl}} \cdot \mathrm{U}_{\mathrm{drift}}\right)$ in the Thomson scattered frequency given by Eq. (12) can be written as,

$$
\Delta \omega_{\text {shift }}=\frac{2 \pi c \Delta \lambda_{\text {shift }}}{\lambda^{2}}=\vec{k}_{f i} \cdot \vec{U}_{d r i f t}=k_{i a} U_{r a d} \cos \Phi
$$

where $\Phi$ is the angle between the ion-acoustic wave vector $\mathbf{k}_{\mathrm{ia}}$ and the radial drift velocity $\mathbf{U}_{\text {rad }}$. Eq. (58) can be rearranged for the radial drift velocity in terms of the Thomson spectral shift in pixels on the reticon array,

$$
U_{\text {rad }}=\frac{4.72 \times 10^{5} \Delta \lambda_{\text {shiff }}(\text { pixels })}{\cos \Phi} \quad \frac{\mathrm{cm}}{\mathrm{s}}
$$

The error in the radial drift velocity measurements results from a \pm 1 pixel uncertainty in the spectral shift when read from the reticon array. The radial drift velocities will be normalized to the sound speed $\left(U_{\mathrm{rad}} / \mathrm{c}_{\mathrm{s}}\right)$ for presentation of the data. This allows for easy location of the radial sonic point in the plasma where $U_{\text {rad }} / c_{s}=1$. The unresolved factor in Eq. (59) is the cosine of the angle $\Phi$ between the ion-acoustic wave vector and the radial drift which can be determined by absolute alignment of the diagnostic beam in the plasma.

Absolute distances in the plasma are measured differently for the axial and radial 


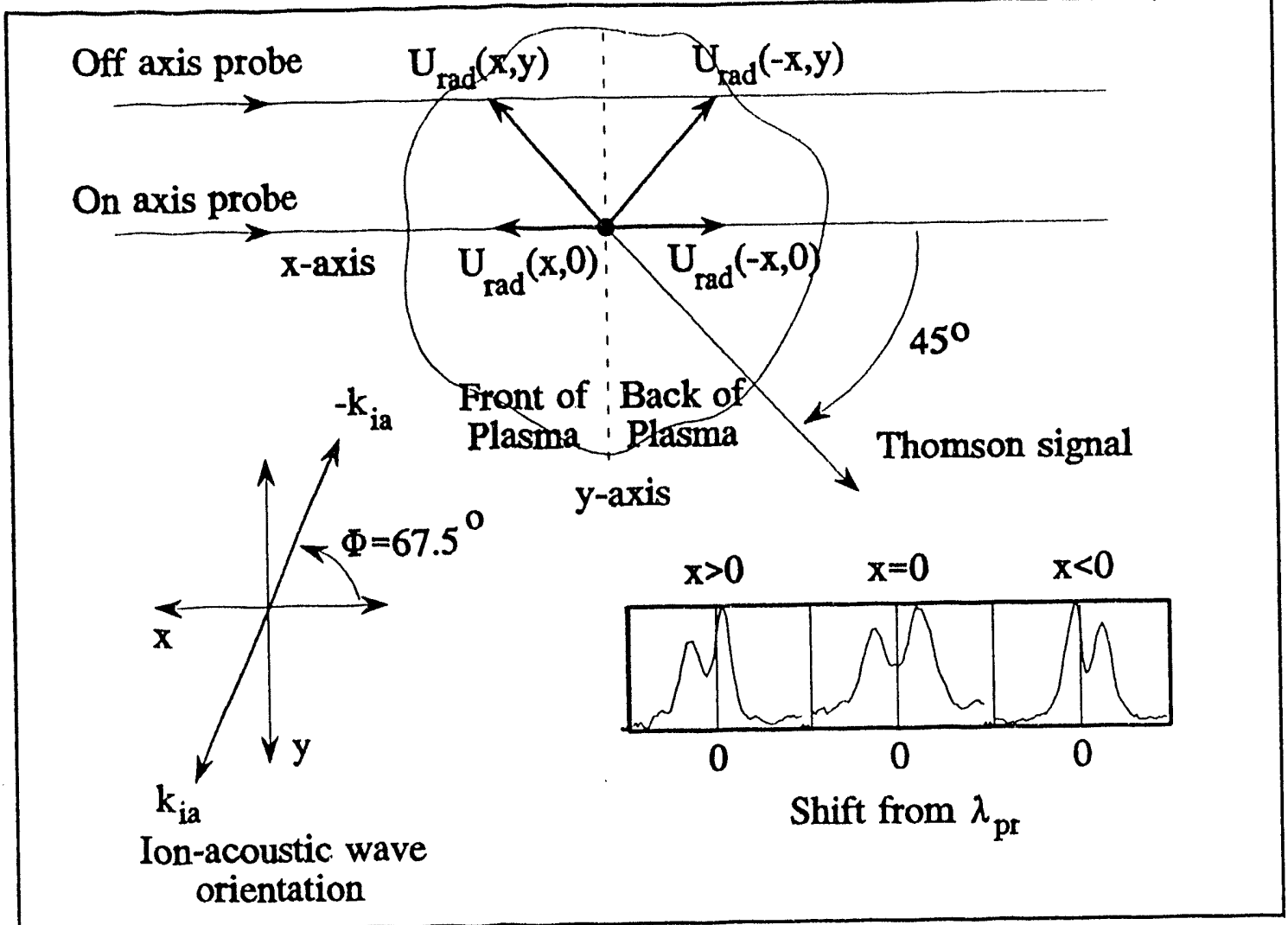

Figure 27: Radial $x-y$ plane at an arbitrary $z$ position in the plasma. Assuming a cylindrically symmetric plasma, an on-axis diagnostic beam will produce a symmetric Doppler shift in the Thomson signal about $x=0$.

directions. In the axial direction, the target surface is used as a reference point. With only the diagnostic beam grazing the target surface, the horizontal slit in the collection system, see Figure 17 , is moved to maximize the scattered signal at $\lambda_{\text {pr. }}$. A spatially sharp maximum occurs when the slit is aligned with the image of the target surface and the accuracy of the position is given by one-half the effective slit width (i.e. $\pm 15 \mu \mathrm{m}$ ). The slit is then moved to the desired axial position and the diagnostic beam is aligned axially with the slit by maximizing the detected Thomson signal. Measurements in the radial direction are performed by translating the image of the plasma across the vertical slit of the spectrometer, see Figure 17, which selects a different portion of the diagnostic beam 
to scatter from. The position of the diagnostic beam in the radial plane can be found through the use of the radial drift velocity measurements. Figure 27 depicts the radial $x-y$ plane $\left(r=\left(x^{2}+y^{2}\right)^{1 / 2}\right)$ at an arbitrary $z$ position in the plasma. Values of $x>0$ refer to the front of the plasma where the diagnostic beam enters and values of $x<0$ refer to the back of the plasma. As seen in the figure, the pair of ion-acoustic wave vectors integrated by the 45 degree scattering geometry are oriented at 67.5 degrees with respect to the $\mathrm{x}$-axis. This orientation is determined by the phasematching condition for Thomson scattering in Eq. (11). The ion-acoustic wave with wave vector $\mathrm{k}_{\mathrm{ia}}$ produces the blue shifted peak in the Thomson spectrum and the ion-acoustic wave with wave vector $-\mathrm{k}_{\mathrm{ia}}$ produces a red shift. Assuming the plasma is cylindrically symmetric, the radial drift velocity is zero at the center of the plasma $(r=0)$ and increases uniformly in all directions away from the center of the plasma. If the diagnostic beam lies on the $x$-axis, then the magnitude of $\cos \Phi$ in Eq. (59) is $\cos (67.5)$ for any position along the diagnostic beam and changes sign from plus to minus when crossing from $x>0$ to $x<0$. In this case, the Doppler shift in the Thomson spectrum will be symmetric when scattering is monitored from a portion of the diagnostic beam to either side of $x=0$. In other words, a red shift will occur towards the back of the plasma for $\mathrm{x}=-\mathrm{x}_{\mathrm{o}}$, an equivalent blue shift for $\mathrm{x}=\mathrm{x}_{\mathrm{o}}$, and no shift will occur for $\mathrm{x}=0$ as depicted by the Thomson spectra shown in Figure 27 . If the probe is off axis, the magnitude of $\cos \Phi$ is not constant along the probe path and its sign will be predominantly plus or minus depending on which side of the $x$-axis the probe is on. For example, the off-axis probe in Figure 27 will produce a red Doppler shift for most of the probe path. The symmetric Doppler shift is used to position the 


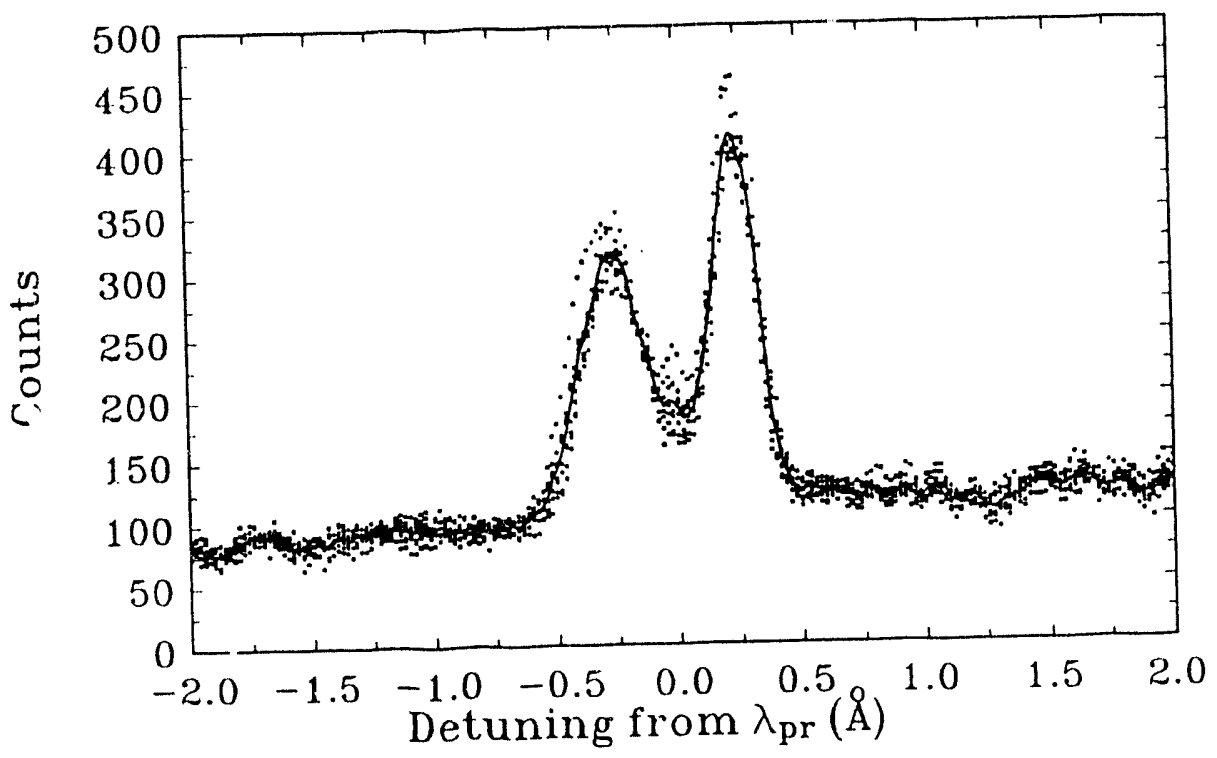

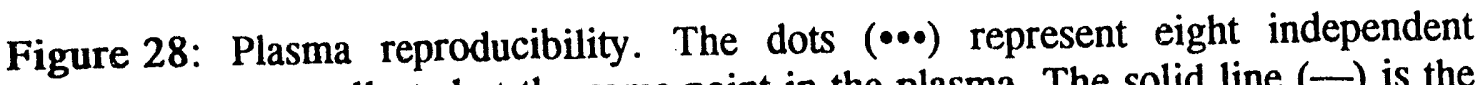
Thomson spectra collected at the same point in the plasma. The solid line $(-)$ is the average of the independent spectra.

diagnostic beam along the $\mathrm{x}$-axis. When the diagnostic beam is coincident with the $\mathrm{x}$-axis, the factor $\cos \Phi$ is a constant in Eq. (59) and the radial drift velocity can be measured directly from the Doppler shift in the spectrum. The z-axis reference is found, with an accuracy of $\pm 15 \mu \mathrm{m}$, when no Doppler shift due to the drift velocity occurs. The existence of a symmetry in the shift proves the plasma is cylindrically symmetric in its radial expansion velocity.

The laser produced plasma generated in this experiment must be reproducible in the context of the three quantities being measured: electron density, sound speed, and radial drift. The reproducibility guarantees that pointwise measurements from different 
plasma shots are correlated. Figure 28 shows the collective Thomson spectrum from an arbitrary point in plasma for eight independent data sets from different plasma shots. The data is given by the dots and the solid line is the average of the eight sets. The absolute fluctuation of the integral of the normalized counts under the spectra (electron density) around the eight set average is $\pm 7 \%$. Therefore, the electron density is reproducible from plasma shot to plasma shot. The sound speed which is proportional to the separation of the peaks is constant from shot-to-shot within the \pm 1 pixel reading error of the peak separation. The radial drift which is proportional to the Doppler shift of the spectrum from the diagnostic wavelength $\lambda_{\mathrm{pr}}$ is also constant from shot-to-shot within the \pm 1 pixel reading error. In this case, the Doppler shift is approximately zero because the diagnostic is aligned with the z-axis of the plasma. These results experimentally verify that the plasma is reproducible on average in the three measured quantities.

The diagnostic beam must also be nonintrusive in order for the data to be meaningful. In other words, inverse bremsstrahlung absorption of the diagnostic beam must be small to avoid local heating in the plasma which would affect the sound speed measurements. The photodiode/pulse height analyzer detection system already indicates that the absorption of the diagnostic beam due to the plasma is small, less than $\approx 4 \%$, in the region of interest. The small absorption is corroborated by the results of the measurement conducted in Figure 29. The measurement involved lowering the diagnostic (probe) intensity by a factor of three while monitoring the peak separation (sound speed) from the Thomson spectra at the same point in the plasma. Since the sound speed does not vary over the full range of probe intensities, the diagnostic is assured to be 


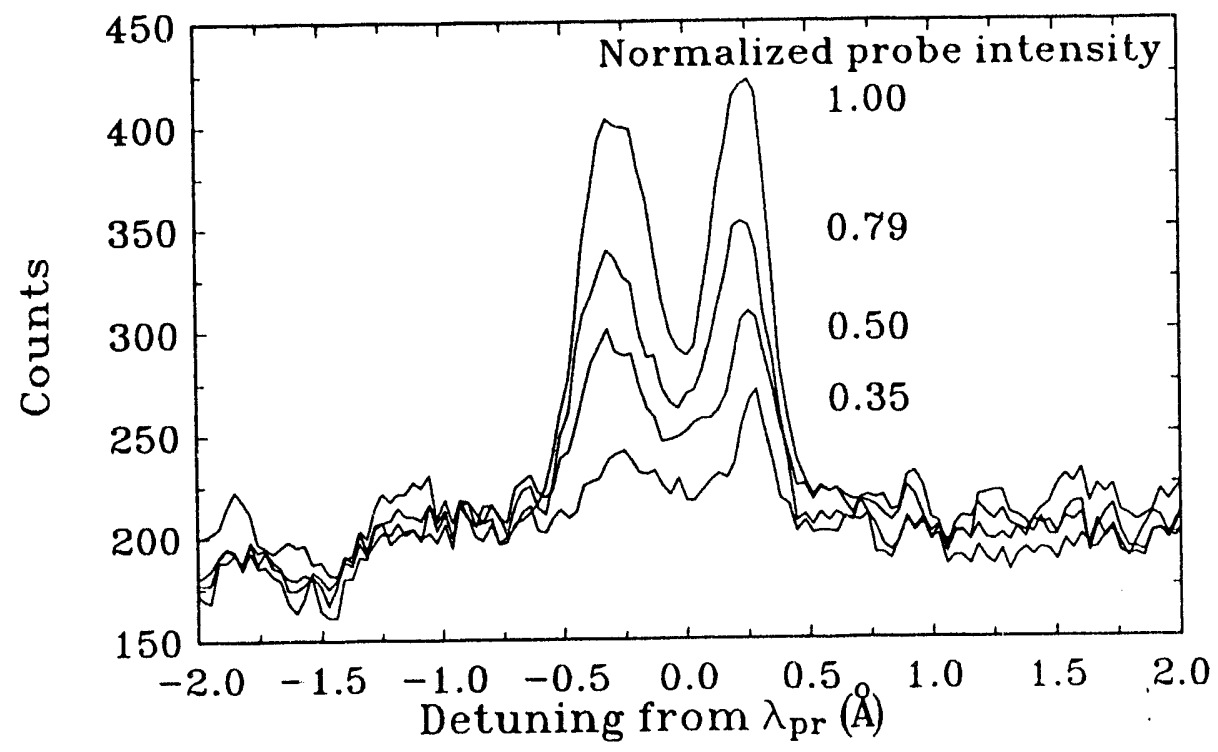

Figure 29: Thomson spectra taken at the same point in the plasma for different diagnostic intensities. The sound speed, peak separation, is independent of diagnostic intensity.

nonintrusive within the measurement errors of the experiment.

The results from the collective Thomson scattering measurements of the laser produced plasma are shown in Figure 30, Figure 31, and Figure 32. The quantities: normalized electron density $\left(n_{e} / n_{c}\right)$ where $n_{c}=1 \times 10^{21} \mathrm{~cm}^{-3}$ is the critical density of the $1.06 \mu \mathrm{m}$ plasma generating beam, sound speed squared $\mathrm{c}_{\mathrm{s}}^{2}=(\mathrm{Z}+5 / 3) \mathrm{T}(\mathrm{eV})$, and normalized radial drift velocity $\left(U_{\mathrm{rad}} / c_{\mathrm{s}}\right)$ are measured in the $\mathrm{x}-\mathrm{z}$ plane in the plasma. The graphs are a result of taking a linear interpolation between data points on a $6 \times 9$ grid, 6 axial points and 9 radial points. Since error bars on the data cannot be displayed in this fashion, the graphs are meant to provide only a global view of the plasma quantities. 


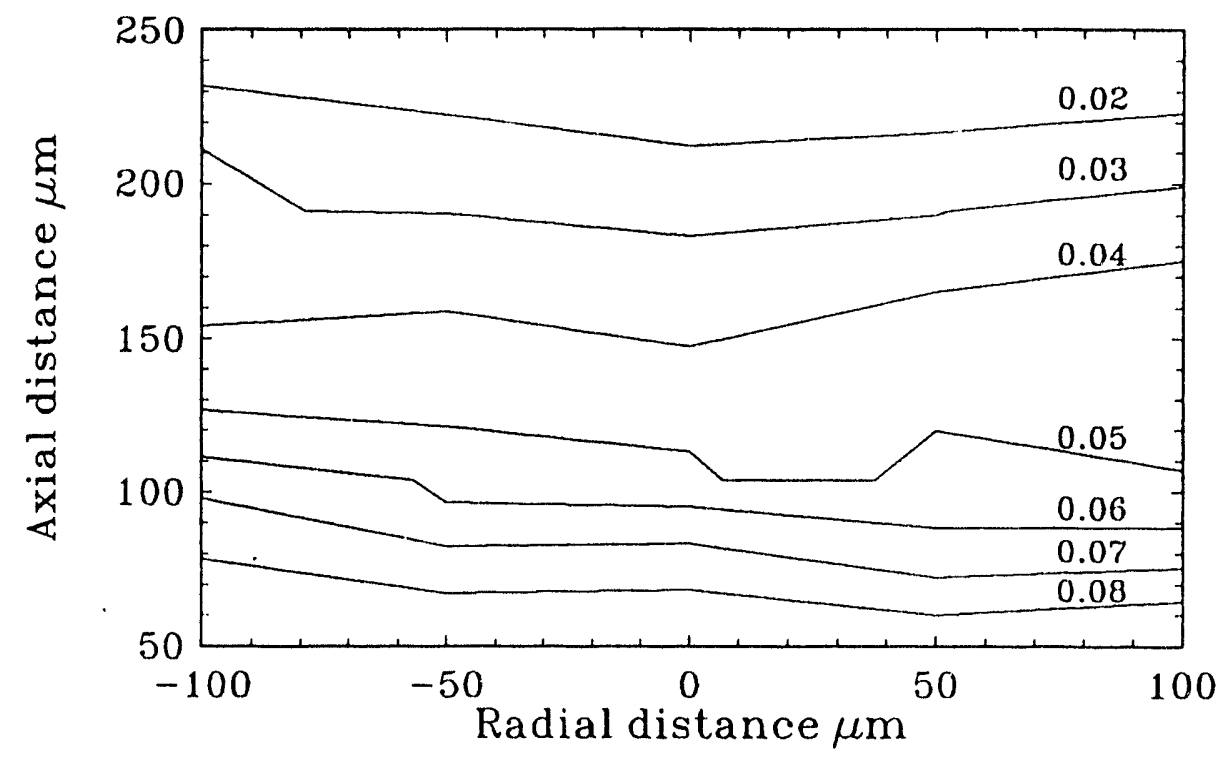

Figure 30: Contour plot of the experimentally measured normalized electron density $\left(n_{\mathrm{e}} / \mathrm{n}_{\mathrm{c}}\right)$ from collective Thomson scattering.

Radial distances $>100 \mu \mathrm{m}$ are not included to limit the number of grid points, and therefore the amount of data to a reasonable level. A scan at $\mathrm{z}=165 \mu \mathrm{m}$ and $\mathrm{r}<350 \mu \mathrm{m}$ is taken for comparison with LASNEX for $\mathrm{r}>100 \mu \mathrm{m}$ and will be presented shortly.

Before a comparison is made between the data and LASNEX, some insight can be gained by applying simple physical modeling to the two-dimensional data. First, the electron density contours of Figure 30 are constant in the radial dimension. This dependence is expected because the measurements are made only from the central portion of the plasma to just outside of the FWHM $=100 \mu$ m plasma generating beam diameter and the spherical nature of the plasma expansion will only become apparent at larger 


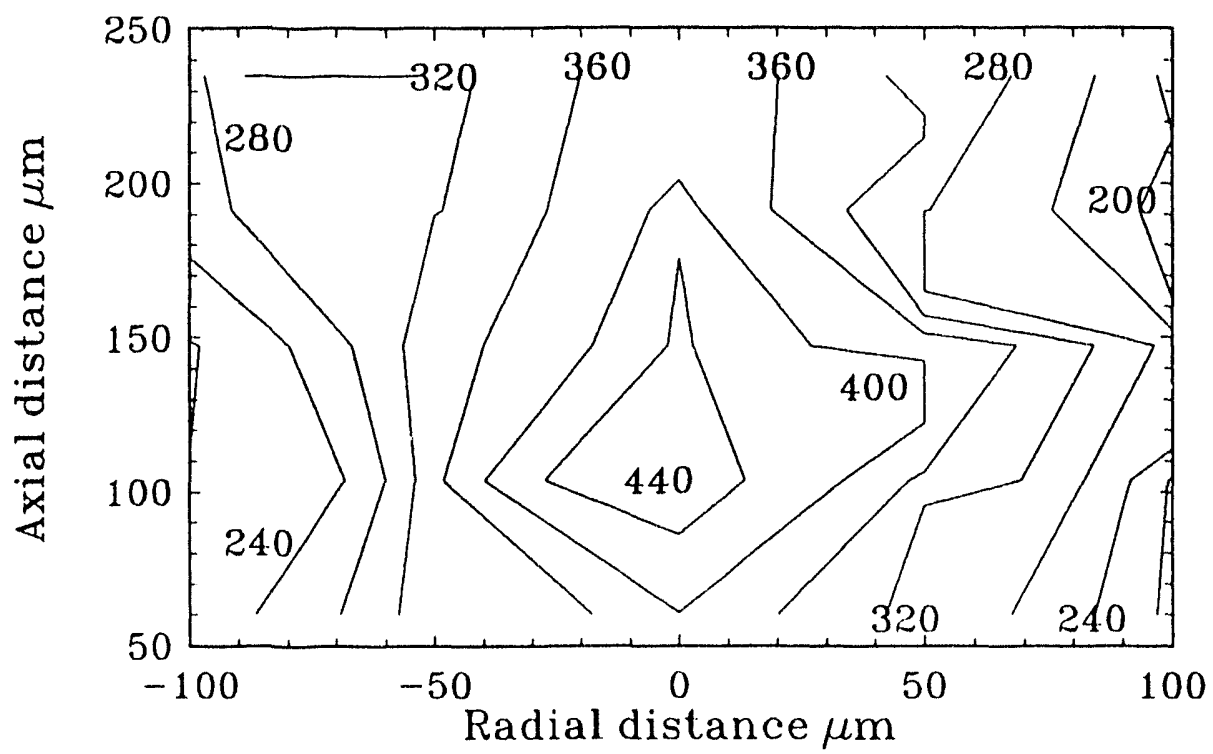

Figure 31: Contour plot of the experimentally measured $(\mathrm{Z}+5 / 3) \mathrm{T}_{\mathrm{e}}(\mathrm{eV})$ product from collective Thomson scattering.

radial distances.

Figure 31 is the contour plot of the product of the two-dimensional average ionization state $(\mathrm{Z})$ and electron temperature $\left(\mathrm{T}_{\mathrm{e}}\right)$. The average ionization is a function of the electron temperature and by applying the appropriate equilibrium model, the two quantities $\mathrm{Z}$ and $\mathrm{T}_{\mathrm{e}}$ can be independently approximated from the data. Assuming the plasma has reached a steady ionization state by the peak of the plasma generating pulse, one of three equilibrium models may be applied: LTE equilibrium model, Coronal equilibrium model, or Ionization balance model. The time $\tau_{\mathrm{e}}$ necessary to reach steady state for an average ionization $\mathrm{Z}$ can be approximated by ${ }^{136}$, 




Figure 32: Contour plot of the experimentally measured normalized radial drift velocity $\left(U_{\mathrm{rad}} / \mathrm{c}_{\mathrm{s}}\right)$ from collective Thomson scattering. The circles are the sonic surface position predicted by modeling.

$$
\tau_{e} \approx \frac{1}{n_{e}} \sum_{i=1}^{Z} \frac{1}{C^{\text {ionize }}\left(i-1, T_{e}\right)}
$$

where $C^{\text {ionize }}\left(\mathrm{Z}, \mathrm{T}_{\mathrm{e}}\right)$ is the electron temperature dependent ionization coefficient for a ion of charge $\mathrm{Z}$ in its ground state. The ionization coefficient is given approximately by (Ref. 136),

$$
C^{\text {ionize }}\left(Z, T_{e}\right)=\frac{9 \times 10^{-6} \xi_{z}\left(T_{e} / \chi_{z}\right)^{1 / 2}}{\chi_{z}^{3 / 2}\left(4.88+\left(T_{e} / \chi_{z}\right)\right)} \cdot \exp ^{\frac{-\chi_{z}}{T_{e}}} \mathrm{~cm}^{3} \mathrm{~s}^{-1}
$$

where the electron temperature and ionization energy of an ion of charge $\mathrm{Z}$ are in (eV) 




Figure 33: Approximate time $\tau_{\mathrm{e}}$ necessary to reach a given ionization state $\mathrm{Z}$ in a plasma with $\mathrm{T}_{\mathrm{e}}=40 \mathrm{eV}$ and a range of electron densities $\left(10^{19}-10^{20} \mathrm{~cm}^{-3}\right)$.

and $\xi_{Z}$ is the number of electrons in the outer most shell corresponding to a charge state Z. For the aluminum plasma studied in this experiment, Eq. (60) and Eq. (61) are used to find the ionization time required to reach a given charge state for an approximate plasma electron temperature of $40 \mathrm{eV}$. Figure 33 shows the results for the range of electron densities $\left(10^{19}-10^{20} \mathrm{~cm}^{-3}\right)$ measured from the contours in Figure 30 for this experiment. Because of the density dependence in Eq. (60), a given charge state at the highest electron density $10^{20} \mathrm{~cm}^{-3}$ will arrive at steady state faster than the lowest electron density $10^{19} \mathrm{~cm}^{-3}$ as seen in the figure. The charge states $(Z \leq 6)$ are reached in less time than the time scale of the plasma generating pulse (9ns) are most likely to be 


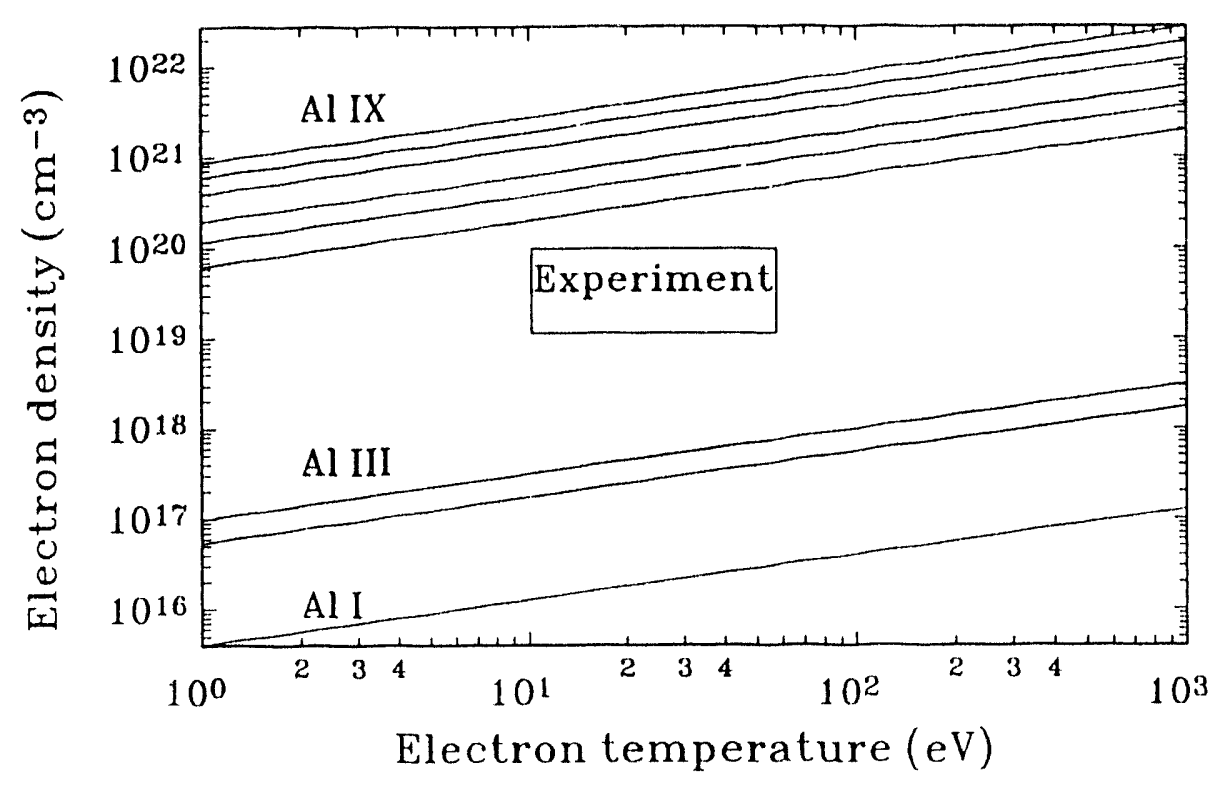

Figure 34: Electron densities required as a function of electron temperature for an aluminum species to be in LTE. An aluminum species is in LTE when the density lies above the line for that species.

in steady state at the peak of the pulse, while the highest charge states $(\mathrm{Z}=7,8)$ generated in this plasma may not be in steady state. However, the plasma charge states are approximately in steady state. The three equilibrium models used to relate the average ionization state to the electron temperature are applicable under different plasma conditions. LTE or local thermodynamic equilibrium ${ }^{137}$ applies to high density plasmas in which the collisional excitation rates of energy levels in a given ion are much faster than the deexcitation rates due to spontaneous emission of a photon. Under these conditions the excited state levels of an ion are populated according to a Boltzmann distribution. The order of magnitude criteria for the plasma electron density in which an 




Figure 35: The temperature dependent average ionization state for an aluminum plasma with $\mathrm{n}_{\mathrm{e}}=10^{19} \mathrm{~cm}^{-3}$ as predicted by the three models 1) LTE, 2) NLTE (ionization balance), and 3 ) coronal.

ion can be in LTE is given by (Ref. 137),

$$
n_{e} \geq 1.34 \times 10^{14} T_{e}^{1 / 2}\left(\Delta E_{n m}\right)^{3} \quad \mathrm{~cm}^{-3}
$$

where the electron temperature $T_{e}$ and energy gap $\Delta E_{n m}$ are in $(e V)$. The energy gap $\Delta E_{n m}$ for an ion is given by the energy difference between the ground state (shell=n) and lowest excited state for which an electron lies in $($ shell $=n+1)$. Using the energy levels for aluminum ions ${ }^{138}$, the criteria for an ion of charge state $\mathrm{Z}$ to be in LTE can be calculated from Eq. (62). Figure 34 shows the results of the calculation for species $\mathrm{Al}$ I - Al IX along with the approximate plasma conditions for this experiment. As seen by 
the figure, neutral through doubly ionized aluminum ions (Al I - Al III) are definitely in LTE for the laser plasma, but species Al IV and higher are only approximately in LTE. The coronal model ${ }^{139}$ holds in the opposite limit of LTE in low density plasmas where radiative deexcitation rates are much larger than collisional excitation rates. The ions the coronal model are all in their ground state and the average ionization state is approximated by, ${ }^{140}$

$$
Z_{\text {ave }} \approx 26\left[\frac{T_{e}}{1+\left(\frac{26}{Z_{A}}\right)^{2} T_{e}}\right]^{1 / 2}
$$

where the electron temperature in is $(\mathrm{keV})$ and $\mathrm{Z}_{\mathrm{A}}$ is the atomic number. Since the plasma for this experiment is almost in LTE, the coronal equilibrium model will not apply. When a plasma lies between the LTE and coronal electron density limits, an intermediate model must be applied and the ionization balance model ${ }^{141}$ can be used for this purpose. The ionization balance model includes all of the appropriate excitation/ionization and deexcitation/recombination processes for the ions without making approximations about their relative magnitudes as is done with the LTE and coronal models. The average ionization state predicted by all three models for the aluminum plasma in this experiment as a function of electron temperature are shown in Figure 35 for a electron density of $\mathrm{n}_{\mathrm{e}}=1 \times 10^{19} \mathrm{~cm}^{-3}$. The LTE and ionization balance model, NLTE in the figure, calculations where graciously done by Y.T. Lee, author of Ref. 141, and the coronal model results are given by Eq. (63). As noted by the figure, the ionization balance (NLTE) and LTE results are identical for electron temperatures in 
which the average ionization less than 3 . This result is expected from the calculations in Figure 34 which show the low lying charge states to be in LTE for the aluminum plasma. For higher electron temperatures the LTE model over estimates the average ionization state from the ionization balance model because ions of higher charge states will not be completely in LTE. The coronal model on the other hand under estimates the ionization state in the aluminum plasma for almost the entire range of electron temperatures. From the arguments above, the ionization balance model seems adequate to relate the average ionization state of the aluminum plasma in this experiment to the electron temperature. Also, the ionization balance model calculations for an electron density of $1 \times 10^{20} \mathrm{~cm}^{-3}$ do not differ appreciably from those values predicted by the model at $n_{e}=1 \times 10^{19} \mathrm{~cm}^{-3}$ in Figure 35. Therefore utilizing the ionization balance model results of Figure 35, the two-dimensional $(Z+5 / 3) \mathrm{T}_{\mathrm{e}}$ data from Figure 31 yields a maximum electron temperature of $45 \mathrm{eV}$ with average ionization of 8.2 for a product $(Z+5 / 3) \mathrm{T}_{\mathrm{e}}=440$, and a minimum electron temperature of $25 \mathrm{eV}$ with average ionization of 6.2 for a product $(Z+5 / 3) \mathrm{T}_{\mathrm{e}}$ $=200$ in the region covered by the two-dimensional contour plots. So as promised much earlier in this chapter, this analysis has shown the average ionization state $\mathrm{Z}$ does lie in the range $5 \leq \mathrm{Z} \leq 8$, and with the two-dimensional electron density data from Figure 30, the approximate range for $\alpha$ is $3 \leq \alpha \leq 15$.

Another physical model can be used to approximate the radial sonic point in the plasma, $\left(U_{\mathrm{rad}} / \mathrm{c}_{\mathrm{s}}=1\right)$. These results can then be compared to the actual position of the sonic point measured by the experiment. The electron and ion steady state equations of motion in the radial direction along the $x$-axis are given by (Ref. 10), 


$$
\begin{gathered}
e E+\frac{1}{n_{e}} \frac{\partial P_{e}}{\partial x}+\mathrm{A} \frac{\partial T_{e}}{\partial x}=0 \\
m_{i} v_{i} \frac{\partial v_{i}}{\partial x}=-\frac{1}{n_{i}} \frac{\partial P_{i}}{\partial x}+Z e E+\mathbf{A} \frac{n_{e}}{n_{i}} \frac{\partial T_{e}}{\partial x}
\end{gathered}
$$

where the e and i subscripts refer to the electron and ion quantities, respectively, $E$ is the electric field, $\mathrm{P}$ is the pressure, $\mathrm{v}$ is the velocity, $\mathrm{m}$ is the mass, and the last term in each equation is the thermal force in which the coefficient $A$ is a slowly varying function of $\mathrm{Z}$ with $\mathrm{A} \approx 1$ (Ref. 10 ). By eliminating the electric field between the two equations, the derivative of the ion velocity can be written in terms of the electron and ion pressures,

$$
\frac{m_{i}}{2} \frac{\partial v_{i}^{2}}{\partial x}=-\frac{1}{n_{i}} \frac{\partial P_{i}}{\partial x}-\frac{Z}{n_{e}} \frac{\partial P_{e}}{\partial x}=-\frac{1}{n_{i}} \frac{\partial\left(P_{i}+P_{e}\right)}{\partial x}
$$

where the last equality is obtained by assuming quasineutrality $\left(\mathrm{n}_{\mathrm{e}}=\mathrm{Zn}_{\mathrm{i}}\right)$. The pressure is related to the density and temperature through the perfect gas law $(P=n T)$, therefore $\left(\mathrm{P}_{\mathrm{e}} / \mathrm{P}_{\mathrm{i}}\right)=\left(\mathrm{n}_{\mathrm{e}} \mathrm{T}_{\mathrm{e}} / \mathrm{n}_{\mathrm{i}} \mathrm{T}_{\mathrm{i}}\right) \approx \mathrm{Z}$ when the electron and ion temperatures are approximately equal. With this approximation and the assumption that the electron density is a constant in the radial direction, a fact corroborated by the contour plot of Figure 30, Eq. (65) can be rewritten to yield,

$$
\frac{m_{i}}{2} \frac{\partial v_{i}^{2}}{\partial x}=-Z \frac{\partial\left(T_{e}(1+1 / Z)\right)}{\partial x}
$$

This equation can be integrated from the center of the plasma $(x=0)$ where $v_{i}=0$ to $\left(x=x_{0}\right)$ where the ion radial drift velocity equals the sound speed $\left(v_{i}=c_{s}\right)$. The integral 
of the term on the right side of the equation cannot be done in general and must be approximated by letting the ionization term equal its average of the interval $0<x<x_{0}$, i.e. $\mathrm{Z} \approx\langle\mathrm{Z}\rangle$,

$$
\begin{gathered}
\frac{m_{i}}{2} \int_{x=0}^{x_{0}} \frac{\partial v_{i}^{2}}{\partial x} d x=\frac{m_{i}}{2} c_{s}^{2}\left(x_{0}\right) \\
=-\int_{x=0}^{x_{0}} Z \frac{\partial\left(T_{e}(1+1 / Z)\right)}{\partial x} d x \approx\left\langle Z>\left[\left(T_{e}\left(x_{o}\right)\left(1+1 / Z\left(x_{o}\right)\right)\right)-\left(T_{e}(0)(1+1 / Z(0))\right)\right]\right.
\end{gathered}
$$

With the sound speed given by $c_{s}^{2} \approx(Z+5 / 3) T_{e} / m_{i}$ for equal electron and ion temperatures Eq. (67) can be rearranged and written in its final form,

$$
T_{e}\left(x_{o}\right)\left[\left(\frac{Z\left(x_{0}\right)+5 / 3}{2}\right)+<Z>\left(1+1 / Z\left(x_{o}\right)\right)\right]=\left\langle Z>(1+1 / Z(0)) T_{e}(0)\right.
$$

This equation relates the electron temperature and ionization state at the radial sonic point to the same two quantities at the center of the plasma. By solving Eq. (68) with the $(\mathrm{Z}+5 / 3) \mathrm{T}_{\mathrm{e}}$ data of Figure 31 and the ionization balance model, the radial position of the sonic point can be predicted and compared to the sonic point $\left(U_{\mathrm{rad}} / \mathrm{c}_{\mathrm{s}}=1\right)$ measured in the actual radial drift velocity data of Figure 32 . The circles in Figure 32 represent the sonic point positions as calculated by Eq. (68). For positive radial positions, the model and the experiment actually agree within the error bars for the data which cannot be displayed on the contour plot. For negative radial positions, the sonic surface lies mostly outside of the experimental region. The predicted points that do fall within $\mathrm{r}>-100 \mu \mathrm{m}$ still agree fairly well with the data. As a final note, for large and constant values of $\mathrm{Z}$ in Eq. (68), thu electron temperature at the sonic surface is simply related to the electron 


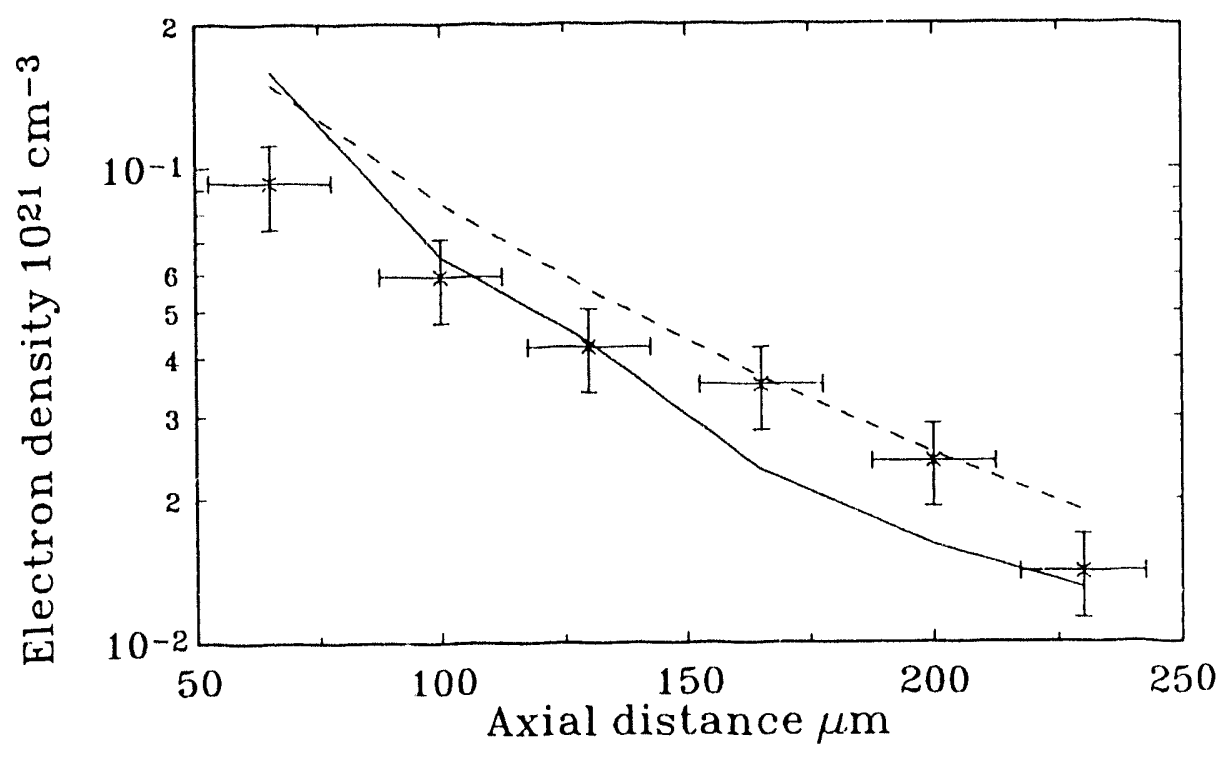

Figure 36: Measured electron density $\left({ }^{*}\right)$ on axis as a function of axial distance. Results from a LASNEX simulation (-) and the analytical spherical model (- - ) are included for comparison.

temperature at the center of the plasma by, $\mathrm{T}_{\mathrm{e}}\left(\mathrm{x}_{\mathrm{o}}\right)=(2 / 3) \mathrm{T}_{\mathrm{e}}(0)$ [J.S. De Groot].

Now that the two-dimensional data has been shown to be consistent with some physical models, it can be compared with LASNEX. LASNEX is a two-dimensional, Lagrangian, hydrodynamic simulation performed in a cylindrical $r-z$ geometry. The time step is on the order of 1 psec. The spatial extent of the plasma is divided into 2000 Lagrangian zones that represent cylindrical macroparticles that move, expand, compress, and change shape according to pressure gradients. The laser that produces the plasma is modeled by several hundred rays that are traced from the (f/50) source through the plasma including refraction, absorption, and possible exit from the problem. For each 


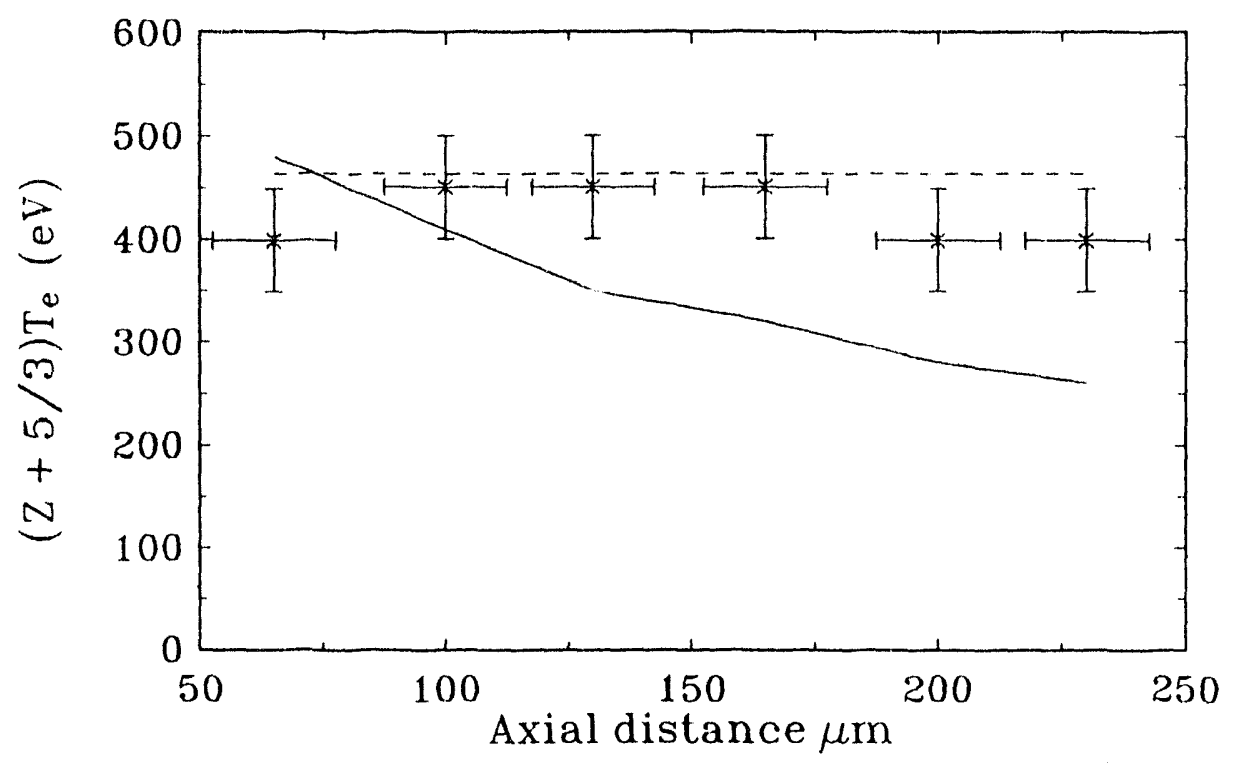

Figure 37: Measured sound speed $\left(^{*}\right)$ on axis as a function of axial distance. Results from a LASNEX simulation $(-)$ and the analytical spherical model (- -) are included for comparison.

time step, laser-light energy absorbed by electrons is transported and equilibrated to ions, radiation, expansion, and compression. Ionization levels are determined by nonlocalthermodynamic-equilibrium models that advance rate equations. ${ }^{142}$ These various models feed back to each other self consistently to form the temperatures, densities, positions, velocities, and opacities for the next time step. LASNEX should model the plasma in this experiment quite well because the electron heat transport is in the Spitzer (Ref. 3) regime, where the electron-ion mean-free path is much less than the temperature scale length so that hydrodynamic fluid equations with classical coefficients should apply. The plasma studied in this experiment can be shown to be in the Spitzer regime by the 




Figure 38: Measured electron density $\left({ }^{*}\right)$ at $\mathrm{z}=165 \mu \mathrm{m}$ as a function of radial distance. Results from a LASNEX simulation at $\mathrm{z}=150 \mu \mathrm{m}(-)$ and $\mathrm{z}=180 \mu \mathrm{m}(--)$ are included for comparison.

appropriate calculation. The electron-ion mean free path $\left(\lambda_{\text {ei }}\right)$ is defined as,

$$
\lambda_{e i}=1.38 \times 10^{13} \frac{T_{e}^{2}}{n_{e} Z \ln \Lambda_{e i}} \mathrm{~cm}
$$

where $n_{e}$ is the electron density in $\left(\mathrm{cm}^{-3}\right), Z$ is the ionization state, $\ln \Lambda_{e i}$ is the Coulomb logarithm for electron-ion interactions, and $\mathrm{T}_{\mathrm{e}}$ is the electron temperature in $(\mathrm{eV})$. The temperature scale length $\left(\mathrm{L}_{\mathrm{T}}\right)$ is defined as, 


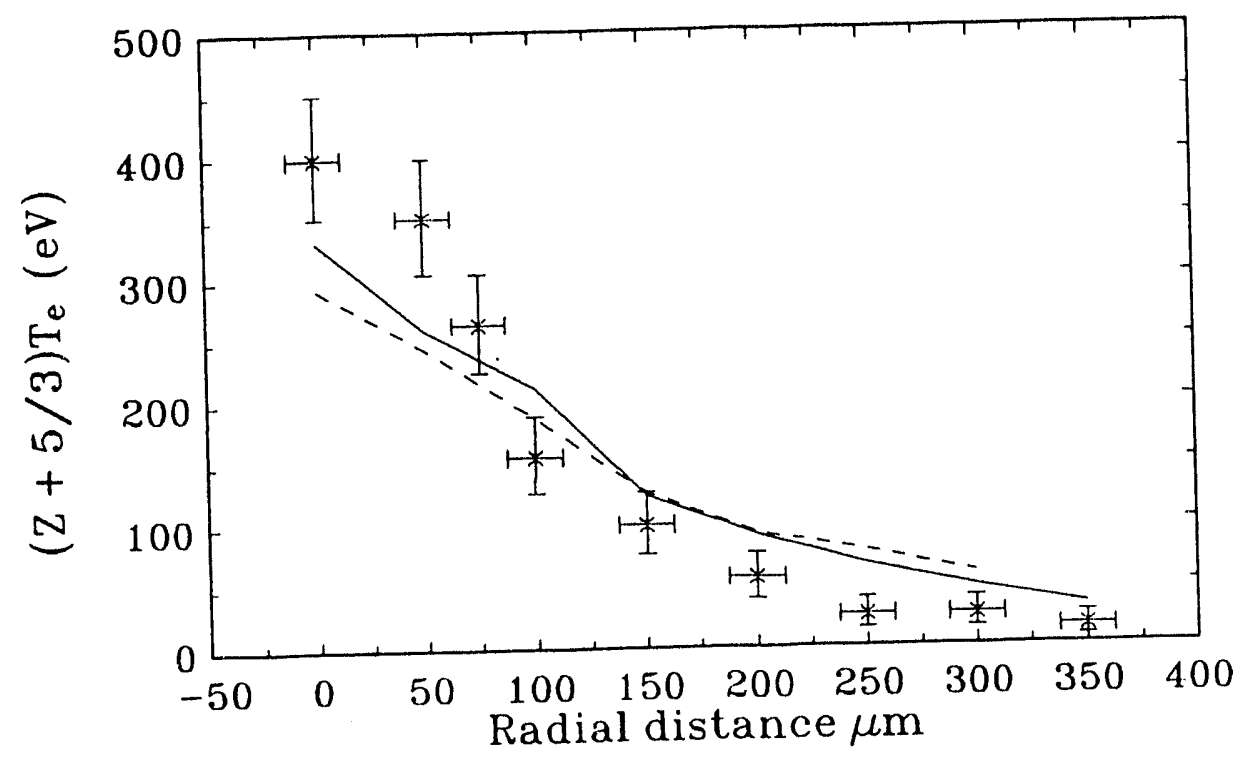

Figure 39: Measured sound speed $\left(^{*}\right)$ at $\mathrm{z}=165 \mu \mathrm{m}$ as a function of radial distance. Results from a LASNEX simulation at $\mathrm{z}=150 \mu \mathrm{m} \mathrm{(-)}$ and $\mathrm{z}=180 \mu \mathrm{m}\left(-\right.$ - $^{-}$) are included for comparison.

$$
L_{T}=\frac{T_{e}}{\left(\partial T_{e} / \partial x\right)}
$$

and has a computed minimum of about $\mathrm{L}_{\mathrm{T}} \approx 60 \mu \mathrm{m}$ at radial positions $75-100 \mu \mathrm{m}$ from the experimental data. The maximum electron-ion mean free path at those positions where $\mathrm{L}_{\mathrm{T}}$ is a minimum is $\lambda_{\mathrm{ei}} \approx 0.3 \mu \mathrm{m}$. Therefore, the criteria for the Spitzer regime $\left(\lambda_{\mathrm{ei}}<\right.$ $\mathrm{L}_{\mathrm{T}} / 100$ ) is satisfied for this plasma. Figure 36 through Figure 40 compare the experimental results with the LASNEX simulation (run\# $211020 \mathrm{~g}$ ) of the plasma. The axial dependence of the electron density and sound speed are shown in Figure 36 and Figure 37 for axial positions $65 \mu \mathrm{m}<\mathrm{z}<235 \mu \mathrm{m}$ and radial position $\mathrm{r}=0$. The radial 




Figure 40: Measured radial drift velocity $\left({ }^{*}\right)$ at $\mathrm{z}=165 \mu \mathrm{m}$ as a function of radial distance. Results from a LASNEX simulation (-) are included for comparison.

dependence of the electron density, sound speed, and radial drift velocity is shown in Figure 38, Figure 39, and Figure 40 at an arbitrary axial position of $\mathrm{z}=165 \mu \mathrm{m}$ and radial positions $0<\mathrm{r}<350 \mu \mathrm{m}$. In Figure 38 and Figure 39 the results from the LASNEX calculation at $\mathrm{z}=150 \mu \mathrm{m}$ and $\mathrm{z}=180 \mu \mathrm{m}$ are displayed to represent the $\pm 15 \mu \mathrm{m}$ uncertainty in the axial position of the experimental data. The radial drift velocity predicted by LASNEX does not vary appreciably from $\mathrm{z}=150 \mu \mathrm{m}$ to $\mathrm{z}=180 \mu \mathrm{m}$. Therefore, only the $\mathrm{z}=165 \mu \mathrm{m}$ calculations from LASNEX are included in the graph of the radial dependence of the radial drift velocity, Figure 40 . The axial and radial electron densities from the LASNEX simulation agree with the experimental data within a percent 
standard deviation of $40 \%$. The axial and radial sound speeds and the normalized radial drift from the LASNEX simulation agree with the experimental data within a percent standard deviation of $50 \%$. The agreement between LASNEX and the experimental data is very good. Therefore, LASNEX has been shown to perform quite well as a simulation code at least in the parameter regime of the plasma in this experiment.

A simple analytical model has been developed that can also be used to understand a laser-heated plasma. This model is an extension of a previous planar model developed ${ }^{143,144}$ for a spherical geometry. This model is one-dimensional and will only apply to measurements made on the z-axis in the axial direction. The plasma is divided into two regions: the conduction region that extends from the ablation surface (essentially the target surface) to the sonic surface, and the corona. The axial fluid velocity is subsonic and the electron temperature increases sharply in the conduction region. The laser light is mainly deposited in the conduction region and the absorbed laser energy is transported by electron conduction to the ablation surface. The axial fluid velocity is supersonic and the electron temperature is almost constant in the corona.

The conduction region for a steady-state, planar plasma extends from the target surface to a distance (Ref. 144),

$$
\Delta_{s s}=0.3\left(\frac{K_{o} T_{s}^{72}}{I_{o}}\right)
$$

where $I_{o}$ is the incident laser energy flux $\left(W / \mathrm{cm}^{2}\right), K_{0}$ is the heat conduction coefficient $\left(\mathrm{K}_{\mathrm{o}}=1.8 \times 10^{29} / \mathrm{Zln}_{\mathrm{ei}} \mathrm{cm}^{-1} \mathrm{sec}^{-1} \mathrm{keV}^{-5 / 2}\right.$, from Ref. 143), and $\mathrm{T}_{\mathrm{s}}$ is the electron temperature at the axial sonic surface $(\mathrm{keV})$. Using the axial sonic surface temperature 
from the LASNEX simulation, the conduction region extends to a distance $\Delta_{\mathrm{ss}} \approx 50 \mu \mathrm{m}$ from the target surface. The planar approximation is approximately valid since the width of the conduction region is less than the full width at half maximum of the laser focal spot $(\mathrm{FWHM} \approx 100 \mu \mathrm{m})$. Because the accessible region of this experiment is limited to axial distances greater than $65 \mu \mathrm{m}$, the measurements in this experiment are limited to the corona.

The time history of the corona is divided into periods: an early planar expansion followed by an approach to spherical steady state. Early in the laser pulse, the plasma expansion is well approximated by a planar, isothermal expansion into vacuum (Ref. 143). The scale length for the plasma density is,

$$
L_{n p}=c_{s} t_{L}
$$

At the peak of the laser pulse, $L_{\mathrm{np}} \approx 190 \mu \mathrm{m}$, where the measured value for the sound speed on axis, $\mathrm{c}_{\mathrm{s}} \approx 4 \times 10^{6} \mathrm{~cm} / \mathrm{s}$, is taken from the experimental data in Figure 31, and $\mathrm{t}_{\mathrm{L}} \approx 0.53 * \mathrm{FWHM}$ of the plasma generating pulse is used. The radial dimension of the plasma is of the order of the full width at half-maximum of the laser focal spot $\approx$ $100 \mu \mathrm{m}$. Thus, since $\mathrm{L}_{\mathrm{np}}>100 \mu \mathrm{m}$, the planar phase of the plasma expansion is over before the peak of the plasma generating pulse.

In a steady-state spherical corona, the electron density is approximately given by (Ref. 144),

$$
n_{e} \approx n_{s}\left(s_{s} / s\right)^{3}
$$

where $n_{s}$ is the electron density at the sonic surface, $s$ is the spherical coordinate and $s_{s}$ 
is the radius of the sonic surface. The usual model for a finite dimensional laser spot assumes a spherical geometry in which the radius of the ablation surface is the full width at half-maximum of the laser focal spot $s_{\mathrm{a}} \approx 100 \mu \mathrm{m}$, so the density scale length at the sonic surface $\left(s_{\mathrm{s}}=\mathrm{s}_{\mathrm{a}}+\Delta_{\mathrm{ss}} \approx 150 \mu \mathrm{m}\right)$ is,

$$
L_{n s} \approx 50 \mu m
$$

The electron density and temperature at the sonic surface are given by (Ref. 144),

$$
\begin{aligned}
& n_{s}=2.2 \times 10^{16} \frac{I_{o}^{1 / 3} A^{1 / 6}}{\left(s_{s} \ln \Lambda_{e i}\right)^{1 / 3} Z^{1 / 2} \lambda^{2 / 3}} \quad \mathrm{~cm}^{-3} \\
& T_{s}=1.1 \times 10^{-6}\left[I_{o} \lambda\right]^{4 / 9}\left[A s_{s} \ln \Lambda_{e i}\right]^{2 / 9} \quad \mathrm{keV}
\end{aligned}
$$

where $I_{0}\left[W / \mathrm{cm}^{2}\right]$ is the intensity of the plasma generating beam, $\lambda[\mu \mathrm{m}]$ is the plasma generating wavelength, $\mathrm{A}$ is the atomic weight of the target material, $\mathrm{Z}$ is the ionic charge state of the plasma, and $s_{\mathrm{s}}$ is the radius of the sonic point $\mathrm{s}_{\mathrm{s}} \approx 0.015 \mathrm{~cm}$. Using the parameters for this experiment, $I_{o} \approx 2 \times 10^{11} \mathrm{~W} / \mathrm{cm}^{2}$ and $\mathrm{Z}=8$ on axis, the sonic surface electron density is $n_{\mathrm{s}} \approx 2 \times 10^{20} \mathrm{~cm}^{-3}$ and the sonic surface electron temperature is $T_{s} \approx 135 \mathrm{eV}$. Equation (76) includes the average energy $\left(E_{\mathrm{i}}\right)$ used per electron for ionization and $E_{i}=130 \mathrm{eV}$ for aluminum with $\mathrm{Z}=8$. Therefore the electron temperature is $\left[\mathrm{T}_{\mathrm{s}}-(2 / 3) \mathrm{E}_{\mathrm{i}}\right] \approx 48 \mathrm{eV}$ at the sonic surface.

Equations (73) through (75) are in good agreement with the axial density data and the LASNEX simulations as shown in Figure 36. The steady-state spherical model predicts that the corona should be isothermal with a temperature equal to the sonic temperature, Eq. (76). With $Z=8$, the predicted $(Z+5 / 3) T_{s}$ product agrees with the 
experimental data and the LASNEX simulations as shown in Figure 37. Thus the steadystate, spherical plasma model appears to be a valid approximation for this experiment. Since the model assumes a steady-state plasma, the good agreement with the experimental data infers that the plasma is approximately stationary during the Thomson scattering experiments. This conclusion is corroborated by the well-defined peaks in the collective Thomson data and a predicted $B_{\text {adj }} \approx 0.9$.

Two questions can now be addressed with the presentation of the experimental data and its comparison with LASNEX: 1) Are the electron and ion temperatures of the plasma approximately equal as assumed throughout the analysis?, 2) Could a 532nm diagnostic beam have been used instead of a $266 \mathrm{~nm}$ diagnostic beam in this experiment? The rate $\left(v^{\mathrm{i} / \mathrm{e}}\right)$ at which the ion temperature will equilibrate with the electron temperature is given by ${ }^{145}$,

$$
v^{i / e}=3.2 \times 10^{-9} \frac{n_{e} Z^{2} \ln \Lambda_{e i}}{A T_{e}^{3 / 2}} \sec ^{-1}
$$

where $\mathrm{A}$ is the atomic weight of the plasma material, for aluminum $\mathrm{A}=27$, the electron density is in $\mathrm{cm}^{-3}$, and the electron temperature is in $\mathrm{eV}$. With the most pessimistic values for $Z, \ln \Lambda_{e i}, n_{e}$, and $T_{e}$ of the plasma that are measured from the experiment (i.e. $Z=5$, $\ln \Lambda_{\mathrm{ei}}=5, \mathrm{n}_{\mathrm{e}}=1 \times 10^{19} \mathrm{~cm}^{-3}$, and $\mathrm{T}_{\mathrm{e}}=45 \mathrm{eV}$ ), the maximum time that it takes the ions to thermally equilibrate with the electrons is $\left(1 / \mathrm{v}^{\mathrm{i} / \mathrm{e}} \approx 2 \mathrm{~ns}\right)$. Since this time is less than the FWHM $=9$ ns width of the plasma generating pulse, the answer to the first question is yes and the ion temperature will approximately be equal to the electron temperature. In fact, LASNEX predicts the electron and ion temperatures to be equal as well. Two factors 
determine whether a diagnostic wavelength will be suitable for Thomson scattering from a plasma: inverse bremsstrahlung absorption and refractive turning. Inverse bremsstrahlung absorption of the diagnostic beam can cause local heating in the plasma and artificially change the sound speed that is being measured. The inverse bremsstrahlung absorption length $\alpha_{b}$ for a beam of frequency $\omega$ is given by Eq. (9) as $\alpha_{b}$ $\approx \omega_{\mathrm{p}}{ }^{2} v_{\mathrm{ei}} / \omega^{2} \mathrm{c}$. The plasma frequency and the electron-ion collision frequency are defined by $\left(\omega_{\mathrm{p}}=5.64 \times 10^{4} \mathrm{n}_{\mathrm{e}}^{1 / 2}\right)$ and $\left(v_{\mathrm{ei}}=3 \times 10^{-6}\left(\mathrm{n}_{\mathrm{e}} \mathrm{Zln} \Lambda_{\mathrm{ei}}\right) / \mathrm{T}_{\mathrm{e}}{ }^{3 / 2}\right)$, respectively, with the electron density in $\mathrm{cm}^{-3}$ and the electron temperature in $\mathrm{eV}$. For a diagnostic beam passing through the central portion of the measurement region of the plasma $(z=130 \mu \mathrm{m})$, the parameters are $\mathrm{n}_{\mathrm{e}} \approx 5 \times 10^{19} \mathrm{~cm}^{-3}, \mathrm{~T}_{\mathrm{e}} \approx 45 \mathrm{eV}, \mathrm{Z} \approx 8$, and $\ln \Lambda_{\mathrm{ei}} \approx 5$. The interaction length (1) is approximately $250 \mu \mathrm{m}$, therefore the predicted inverse bremsstrahlung absorption $\left\{1-\exp \left(-\alpha_{b} 1\right)\right\}$ on the $532 \mathrm{~nm}$ and $266 \mathrm{~nm}$ diagnostic beams is $5 \%$ and $20 \%$, respectively. The calculated $5 \%$ absorption on the $266 \mathrm{~nm}$ diagnostic beam is in line with the less than $4 \%$ absorption measured in the experiment. The $20 \%$ absorption exhibited by a $532 \mathrm{~nm}$ diagnostic beam is large and might begin to affect the sound speed in the plasma. The refractive turning of the two diagnostic wavelengths in the plasma have been calculated by post processing the data from the LASNEX simulation [thanks to M.K. Prasad]. Since the LASNEX simulation has been shown to fit the experimental data, the refractive turning calculations should be fairly accurate. Figure 41 displays the results of the calculation as a function of axial distance of the diagnostic beams from the target surface. The refractive turning angle should be kept smaller than the acceptance angle of the $(\mathrm{f} / 12)$ Thomson collection lens used in this experiment and 




Figure 41: Refractive turning calculations for a $532 \mathrm{~nm}$ and $266 \mathrm{~nm}$ diagnostic beam as a function of axial distance in the laser plasma. The calculations are based on the LASNEX simulation results for the plasma.

the acceptance angle of 2.38 degrees is shown in Figure 41 for comparison. As seen by the figure, the refraction of the $532 \mathrm{~nm}$ diagnostic beam is greater than or equal to the acceptance angle for a fair amount of the region measured in the plasma $(65 \mu \mathrm{m}<\mathrm{z}<$ $130 \mu \mathrm{m}$ ), and the refraction of the $266 \mathrm{~nm}$ diagnostic beam is always less than the acceptance angle over the entire measurement region $(65 \mu \mathrm{m}<z<235 \mu \mathrm{m})$. Therefore, based on the inverse bremsstrahlung and refractive turning calculations, the answer to the second question is no and a 532nm diagnostic wavelength would not be suitable for conducting collective Thomson scattering experiments on the laser-produced plasma studied in this dissertation. 
Despite the self-consistency of the measurements of electron density, sound speed, and radial drift velocity in the laser produced plasma and the excellent agreement of the experimental data with the LASNEX simulation and simple analytical model, discrepancies exist. As mentioned in Chapter 2, a peak height asymmetry in the collective Thomson spectrum is a result of a relative thermal drift between the electrons and ions in the plasma. The most probable source for the drift is the return current driven by heat transport in the plasma, although other mechanisms such as beam nonuniformities could be important. In the Spitzer regime, the relative drift vector $\left(U_{d}\right)$ is related to the heat flux vector $q$ by the following relationship ${ }^{146}$,

$$
\vec{U}_{d}=-\frac{9 \pi}{640}\left(\frac{\gamma_{T}}{\gamma_{E}{ }^{\delta} T^{\varepsilon}}\right) \frac{\vec{q}}{n_{e} T_{e}}
$$

where the factor containing the Spitzer transport coefficients ${ }^{147}, \gamma_{\mathrm{T}}, \gamma_{\mathrm{E}}, \delta_{\mathrm{T}}$, and $\epsilon$, is a slowly varying function of the ionization state $\mathrm{Z}$ and only changes from a value of 5 for $Z=1$ to a value of 2.5 for $Z \rightarrow \infty$. The heat flux vector, in turn, is related to the temperature gradient in the plasma by,

$$
\vec{q}=-\kappa^{e} \vec{\nabla} T_{e}
$$

where $\kappa^{\mathrm{e}}$ is the electron thermal conductivity. Combining Eq. (78) and Eq. (79), the relative drift vector is found to be proportional to the temperature gradient in the plasma. In the radial plane of the plasma in this experiment, the temperature gradient is directed towards the center of the plasma $(r=0)$ from the outside and has a maximum around $\mathrm{r} \approx 100 \mu \mathrm{m}$. The relative drift will be pointed in the same direction as the temperature gradient, towards $(r=0)$. Based on this argument, the product of the ion-acoustic wave 


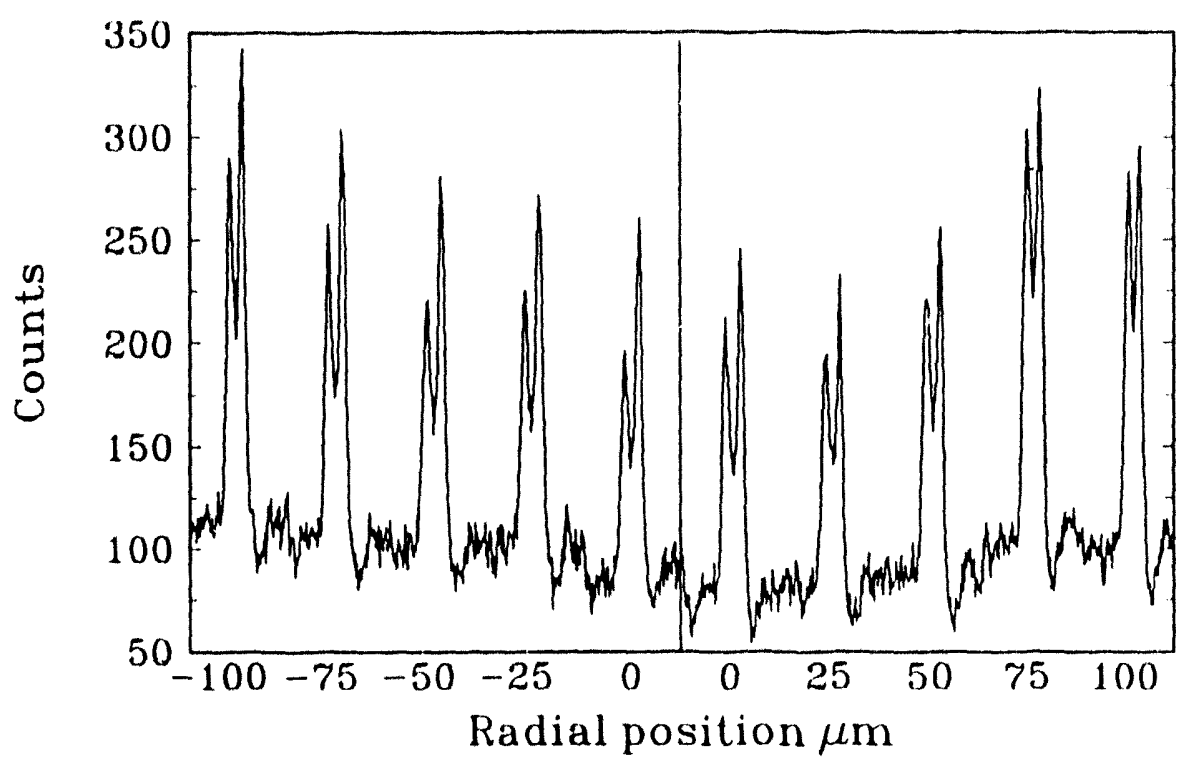

Figure 42: Raw collective Thomson scattering data showing inconsistent peak height asymmetries for different radial positions at an axial distance of $z=200 \mu \mathrm{m}$.

vector with the drift vector $\left(\mathrm{k}_{\mathrm{ia}} \cdot \mathrm{U}_{\mathrm{d}}\right) / \mathrm{kv}_{\mathrm{e}}$ will change sign while passing from negative radial positions $(r<0)$ to positive radial positions $(r>0)$ in this experiment. The product should be zero at $\mathrm{r}=0$ because the gradient of the temperature in the radial direction is zero at $r=0$, and the product should be a maximum around $r \approx 100 \mu \mathrm{m}$ where the temperature gradient is a maximum. Since the magnitude and direction of the peak height asymmetries in the collective Thomson spectrum are proportional to the product $\left(k_{\mathrm{ia}} \cdot \mathrm{U}_{\mathrm{d}}\right) / \mathrm{kv}_{\mathrm{e}}$, see Chapter 2 , the Thomson spectra from this experiment should show equal peak heights at $\mathrm{r}=0$ with a gradual increase in the asymmetry to a maximum at $\mathrm{r} \approx 100 \mu \mathrm{m}$. One of the Thomson peaks should be consistently higher for negative radial 
values while the opposite should hold for positive radial values. As shown in Figure 42, the analysis presented does not hold in general for the data taken in this experiment. Figure 42 is a graph of the raw data taken for the radial positions at an axial distance $z=200 \mu \mathrm{m}$. The left peak in the Thomson spectrum should be lower than the right for $r<0$ and higher than the right for $r>0$. Also at $r=0$, the peak heights should be identical. Obviously this is not the case for the data presented in Figure 42, and for all the data taken in the experiment, the peak height asymmetries appeared to have little correlation with the measured temperature gradient in the plasma. This apparent inconsistency deserves further investigation.

Since measurement of the peak height asymmetry involves line shape analysis of the Thomson spectrum, and the line shape is sensitive to the collisionality $\left(\mathrm{k}_{\mathrm{ia}} \lambda_{\mathrm{ii}}\right)$, a new theory is needed for Thomson spectrum analysis with general values of $k_{\mathrm{ia}} \lambda_{\mathrm{ii}}$. This new theory is important because the existing theories presented in Chapter 2 are only applicable in the small $\mathrm{k}_{\mathrm{ia}} \lambda_{\mathrm{ii}} \rightarrow 0$ and large $\mathrm{k}_{\mathrm{ia}} \lambda_{\mathrm{ii}} \rightarrow \infty$ limits of collisionality of the ionacoustic wave and the values for $\mathrm{k}_{\mathrm{ia}} \lambda_{\mathrm{ii}}$ based on the experimental measurements in this chapter are $\mathrm{k}_{\mathrm{ia}} \lambda_{\mathrm{ii}} \approx 0.1$. This new theory is presented in Chapter 5 and will allow a direct measurement of the relative drift $U_{d}$ from the peak height asymmetries. Along the theory, the experimental configuration must be changed to perform meaningful measurements relating to the peak height asymmetry. Due to the stochastic nature of the peak asymmetries, the relative drift may not be a reproducible quantity in the plasma. Therefore, the experiment should be performed on a single shot basis. Single shot detection of a Thomson signal will require a more sensitive detector than the reticon array 
used for the data acquisition on this chapter. Also, because of the questionable reproducibility of the relative drift, multiple radial positions should be measured at the same time to provide a single shot measurement of the radial temperature gradient for comparison. These issues will be addressed in Chapter 6 along with a presentation of the experimental configuration needed to begin a peak height asymmetry analysis.

This chapter has presented in some detail the data acquisition and analysis of the two-dimensional electron density, sound speed, and radial drift data from $266 \mathrm{~nm}$ collective Thomson scattering in a collisional laser-produced plasma. Accurate measurement of the three plasma quantities is possible with a well-resolved, Rayleighcalibrated, collection system and a high-repetition rate, highly reproducible plasma. The use of a $266 \mathrm{~nm}$ diagnostic beam is necessary to reduce the intrusive effects of inverse bremsstrahlung absorption and the complicating effect of refractive turning which can reduce the collection efficiency of the scattered signal. With some simple but physically based models, the experimentally obtained plasma quantities are shown to be selfconsistent and the electron density, sound speed, and radial drift are used to benchmark the simulation code LASNEX in the parameter regime of the plasma in this experiment. LASNEX is shown to perform fairly well with percent standard deviations from the measured data less than $50 \%$. A simple spherical model is also shown to be a valid approximation for the experimental data and predicts that the plasma is approximately stationary over the width of the diagnostic pulse. This conclusion is experimentally corroborated by the well-defined peaks in the collective Thomson data and a predicted $\beta_{\mathrm{adj}} \approx 0.9$. Despite the self-consistency of the experimental data and good agreement 
between the measurements and simulations, the peak height asymmetries in the collective Thomson spectrum are inconsistent and do not correlate with the measured radial temperature gradients in the plasma. This indicates that although the electron density, sound speed, and radial drift velocity are reproducible and well behaved from shot to shot, the relative thermal drift $\left(U_{d}\right)$ between the electrons and ions may not be reproducible. The investigation of the peak height asymmetry issue and how it relates to the relative thermal drift $\left(U_{d}\right)$ will be conducted in Chapter 5 and Chapter 6 . 
Chapter V: Thomson scattering theory - intermediate ion-ion collisional regime

In Chapter II, the existing theory for analyzing Thomson scattered spectra from a plasma was presented. The present theory, as it stands, only applies to scattering from waves that can be considered either in the collisionless limit $\left(k_{\mathrm{f}} \lambda_{\mathrm{ii}} \rightarrow \infty\right)$ or in the collisional limit $\left(\mathrm{k}_{\mathrm{fl}} \lambda_{\mathrm{ii}} \rightarrow 0\right)$. Although the Thomson scattering experiments in Chapter 4 were conducted from ion-acoustic waves in the intermediate collisional range $\left(\mathrm{k}_{\mathrm{ia}} \lambda_{\mathrm{ii}} \approx\right.$ 1), it was shown in Chapter 2 that the collisionless and collisional theories were adequate for obtaining the electron density, sound speed, and radial drift velocity from the ionacoustic Thomson spectra. Unlike the electron density, sound speed, and radial drift measurements, the measurement of the relative drift velocity $\left(U_{d}\right)$ between the thermal electrons and ions relies on knowledge of the spectral distribution in the ion-acoustic feature, i.e. the peak height asymmetry. As shown in Chapter 2, the spectral distribution of the ion-acoustic feature changes dramatically from the collisionless to collisional limits, see Figure 7, and the exact relationship between the peak height asymmetry and the relative drift velocity will change as well between the two limits. Therefore, the existing theories cannot be used to obtain the relative drift velocity from the peak height asymmetries in the Thomson spectrum for intermediate collisional ion-acoustic waves $\left(k_{\mathrm{ia}} \lambda_{\mathrm{ii}} \approx 1\right)$. This chapter will present a model based on the Fokker-Planck equation coupled with modified fluid equations that will accurately predict the peak height asymmetry in an ion-acoustic Thomson spectrum given the values for the relative drift $\left(\mathrm{U}_{\mathrm{d}}\right)$, ionization/temperature ratio $\left(\mathrm{ZT}_{\mathrm{e}} / \mathrm{T}_{\mathfrak{i}}\right)$ and collisionality of the ion-acoustic wave 
$\left(\mathrm{k}_{\mathrm{ia}} \lambda_{\mathrm{ii}}\right)$. The approach to developing the model first involves defining general closure relations for the linearized ion fluid equations which can be applied for any value of collisionality $\left(\mathrm{k}_{\mathrm{ia}} \lambda_{\mathrm{ii}}\right)$. The ion fluid equations are then used to find the ion susceptibility as was done in Chapter 2 . The dynamical form factor $S(k, \omega)$ which describes the spectral distribution of the Thomson spectra is then known because it is a function of the ion susceptibility. Application of the model requires knowledge of the collisionally dependent transport coefficients that appear in the closure relations. Since the transport coefficients can be calculated with the use of the perturbed ion distribution function $\left(f_{1}\right)$, the linearized ion Fokker-Planck equation will be used to solve for $f_{1}$ assuming collisionless electrons. In obtaining the perturbed ion distribution function, the linearized ion FokkerPlanck equation will be treated as an eigenvalue problem which represents a new and better approach to solving the equation reported in previous publications. In addition to allowing for accurate measurements of the relative drift from the peak height asymmetry in the Thomson spectrum, the model developed in this chapter has the potential of describing the entropy wave feature in the Thomson spectrum which was introduced in Chapter 2.

An obvious, but possibly overlooked fact, is that any one or combination of the fluid equations given in Eq. (42) is equivalent to the basic plasma kinetic equation (Fokker-Planck equation). The reason for this relationship is that the fluid equations are simply moments of the Fokker-Planck equation. Since the Fokker-Planck equation in the collisionless limit $\left(\mathrm{k}_{\mathrm{fl}} \lambda_{\mathrm{ii}} \rightarrow \infty\right)$ becomes the Vlasov equation (Eq. (34)) and predicts Landau damping, so can the fluid equations if properly applied. In fact, the fluid 
equations can be made valid over the entire range of collisionality $\left(\mathrm{k}_{\mathrm{fl}} \lambda_{\mathrm{ii}}\right)$ by using the proper set of general closure relations. Chang \& Callen ${ }^{148}$ use a ChapmanEnskog ${ }^{149}$ like approach to derive a general set of closure relations for the perturbed stress tensor $\left(\pi_{1}\right)$ and heat flux $\left(q_{1}\right)$ in terms of the perturbed flow velocity $\left(u_{1}\right)$ and temperature $\left(T_{1}\right)$ for the ions. These relations are given by,

$$
\begin{gathered}
q_{1}=-\kappa_{1} m_{i} n_{o} v_{i}^{2} u_{1}-i \kappa_{2} n_{o} v_{i} T_{1} \\
\pi_{1}=-i \mu_{1} m_{i} n_{o} v_{i} \mu_{1}-\mu_{2} n_{o} T_{1}
\end{gathered}
$$

where the real quantities $\kappa_{1}, \kappa_{2}, \mu_{1}, \mu_{2}$ are pseudo transport coefficients normalized to $\mathrm{k}_{\mathrm{fl}} \lambda_{\mathrm{ii}}$, and the perturbed quantities are a result of expressing the total stress tensor ( $\pi$ ), heat flux (q), flow velocity $(u)$, and temperature $(T)$ in the form $h=h_{O}+h_{1} \exp (i(k z-$ $\omega t)$ ), where perturbed quantity $\left(h_{1}\right)$ is much less than the background quantity $\left(h_{O}\right), h_{1} / h_{O}$ * 1 . It will be shown in the ion-collisional limit $\left(k_{\mathrm{fl}} \lambda_{\mathrm{ii}} \rightarrow 0\right)$ that $\kappa_{1}$ and $\mu_{2}$ go to zero, while $\kappa_{2}$ and $\mu_{1}$ approach the familiar Braginskii ion thermal conductivity $\left(\kappa_{2} \rightarrow\right.$ $\left.3.9 \mathrm{k}_{\mathrm{f} 1} \lambda_{\mathrm{ii}}\right)$ and ion viscosity $\left(\mu_{1} \rightarrow(4 / 3) 0.96 \mathrm{k}_{\mathrm{fl}} \lambda_{\mathrm{ii}}\right)$. In the ion-collisionless limit $\left(\mathrm{k}_{\mathrm{f} 1} \lambda_{\mathrm{ii}} \rightarrow\right.$ $\infty)$, the viscosity coefficients $\mu_{1}$ and $\mu_{2}$ go to zero while the conductivity coefficients $\kappa_{1}$ and $k_{2}$ remain finite. Chang \& Callen derive expressions for the transport coefficients which are complicated functions of the plasma dispersion $\mathrm{Z}$ function and the Chandrasekhav function, see Ref. 148, and therefore are not easily applied. Chang \& Callen also show that the transport coefficients can be greatly simplified by approximating them as a single function of the $\mathrm{Z}$ function or its derivative of modified argument. Although more tractable, this simplification does not guarantee any degree of accuracy for computing the transport coefficients. The proper solution to the problem of calculating 
the transport coefficients accurately is to first express the perturbed quantities $u_{1}, T_{1}, q_{1}$, and $\pi_{1}$ from Eq. (80) in terms of the perturbed ion distribution function $\left(f_{1}\right)$. The standard definitions for the flow velocity, heat flux, and stress tensor is in terms of a moment of the total distribution function ( $f$ ) are given by,

$$
\begin{gathered}
\vec{u}=\frac{1}{n} \int d^{3} v \vec{v} f\left(v^{\prime}\right) \\
\vec{q}=\frac{m_{i}}{2} \int d^{3} v^{\prime} \vec{v}^{\prime} v^{\prime 2} f\left(v^{\prime}\right) \\
\vec{\pi}=\left[m_{i} \int d^{3} v^{\prime} \vec{v}^{\prime} \vec{v}^{\prime} f\left(v^{\prime}\right)\right]-n T
\end{gathered}
$$

where $v^{\prime}=v-u$, and $n=\int d^{3} v f\left(v^{\prime}\right)$. The temperature is also defined in terms of the of a moment of the total distribution function (f),

$$
T=\frac{(\Gamma-1) m_{i}}{2 n} \int d^{3} v^{\prime} v^{\prime 2} f(v)
$$

This equation is not the standard definition for temperature because the usual factor of $1 / 3$ is replaced with $(\Gamma-1) / 2$, where $\Gamma$ is the ratio of the specific heats $\Gamma=\left(C_{p} / C_{v}\right)$ defined by,

$$
\Gamma=\left|1+2 \frac{\int d^{3} v\left(v_{z}^{2}-1\right) f_{1}(v)}{\int d^{3} v\left(v^{2}-3\right) f_{1}(v)}\right|
$$

The definitions in Eq.'s (84) and (85) are necessary for applying the model developed in this chapter to arbitrary collisionality $\left(k_{\mathrm{fl}} \lambda_{\mathrm{ii}}\right)$. Equations (81) through (84) can be linearized by writing the distribution function as $f=f_{O}+f_{1} \exp (i(k z-\omega t))$ with $f_{1} / f_{O}$ * 
1. Upon substitution, the expressions for the perturbed quantities $u_{1}, T_{1}, q_{1}, \pi_{1}$ are then found to be,

$$
\begin{gathered}
u_{1}=\frac{1}{n_{O}} \int d^{3} v v_{z} f_{1}(v) \\
T_{1}=\frac{(T-1) m_{i} v_{i}^{2}}{2 n_{O}} \int d^{3} v\left(v^{2}-3\right) f_{1}(v) \\
q_{1}=\frac{m_{i} v_{i}^{3}}{2} \int d^{3} v\left(v^{2}-5\right) v_{z} f_{1}(v) \\
\pi_{1}=\left[m_{i} v_{i}^{2} \int d^{3} v v_{z}^{2} f_{1}(v)\right]-n_{o} T_{1}
\end{gathered}
$$

With the perturbed ion distribution function $f_{1}$, equations (85) through (89) can be combined with the complex closure relations of Eq. (80) to find the transport coefficients $\kappa_{1}, \kappa_{2}, \mu_{1}$, and $\mu_{2}$

Invoking the closure relations of Eq. (80), the ion susceptibility $\left(\chi_{\mathrm{i}}\right)$ is modified from the form appearing in Eq. (44) of Chapter 2. As in Chapter 2 the ion fluid equations can be linearized to yield,

$$
\begin{gathered}
-i \omega n_{1}+i k n_{o} u_{1}=0 \\
-i \omega m_{i} n_{o} u_{1}=-i k T_{o} n_{1}-i k n_{o} T_{1}-i k \pi_{1}-i Z e k n_{o} \phi_{1} \\
-\frac{1}{(\Gamma-1)} i \omega n_{o} T_{1}+i k n_{o} T_{o} u_{1}=-i k q_{1}
\end{gathered}
$$

where the factor of $(3 / 2)$ from the linearized fluid equations in Chapter 2, Eq. (43), has been replaced with $(1 /(\Gamma-1))$ to allow for general application of the equations for any collisionality $\left(\mathrm{k}_{\mathrm{fl}} \lambda_{\mathrm{ii}}\right)$. The factor $(1 /(\Gamma-1))$ reduces to $(3 / 2)$ in the collisional limit $\left(\mathrm{k}_{\mathrm{fl}} \lambda_{\mathrm{ii}}\right.$ 
$\rightarrow 0)$ where $\Gamma=5 / 3$ and is equal to $(1 / 2)$ in the collisionless limit $\left(k_{f 1} \lambda_{i i} \rightarrow \infty\right)$ where $\Gamma=3$. The linearized fluid equations, Eq. (90), coupled with the closure relations, Eq. (80), can be solved for the ratio $\left(n_{1} / \phi_{1}\right)$ which in turn can be used to find the ion susceptibility $\left(\chi_{\mathrm{i}}\right)$ as in Chapter 2 ,

$$
\chi_{i}=-\frac{4 \pi Z e}{k^{2}} \frac{n_{1}}{\phi_{1}}=-\frac{Z T_{e}}{T_{i}} \alpha^{2} \frac{x_{i}+i \gamma k_{2}}{x_{i}^{3}+i\left(\mu_{1}+\gamma \kappa_{2}\right) x_{i}^{2}-\left(\gamma\left(1-\mu_{2}\right)\left(1-\kappa_{1}\right)+1+\gamma \mu_{1} k_{2}\right) x_{i}-i \gamma \kappa_{2}}
$$

where $x_{i}=\omega / k v_{i}$ is the normalized frequency and $\gamma=\Gamma-1$. The model which is being developed in this chapter and which is based on the modified form of the ion susceptibility will be referred to as the modified fluid model. For simplicity, the electrons will be assumed collisionless $\left(\mathrm{k}_{\mathrm{fl}} \lambda_{\mathrm{ei}}, \mathrm{k}_{\mathrm{fl}} \lambda_{\mathrm{ee}}\right.$ "1), and the electron susceptibility under this collisionless condition is given by Eq. (37),

$$
\chi_{e}=\alpha^{2}\left[1+x_{e} Z\left(x_{e}\right)\right]
$$

where $\mathrm{x}_{\mathrm{e}}=\omega / \mathrm{kv}_{\mathrm{e}}$ is the normalized frequency and $\mathrm{Z}$ is the plasma dispersion function introduced in Eq. (37) of Chapter 2. A relative drift $\left(U_{d}\right)$ between the thermal electrons and ions can be easily added to the analysis by replacing the normalized frequency $x_{e}$ with $x_{e}+\left(k_{f 1} \cdot U_{d}\right) / k_{f 1} v_{e}$ in Eq. (92). Also, the complex frequency for the normal modes of the plasma can be found by solving for the roots of the dielectric function $\epsilon=0$, where $\epsilon=1+x_{\mathrm{e}}+x_{\mathrm{i}}$

The dynamical form factor $S(k, \omega)$ which predicts the spectral distribution in the Thomson spectrum is defined in terms of the electron and ion susceptibilities, 


$$
S(\vec{k}, \omega)=\frac{2}{\omega \alpha^{2}}\left|\frac{\left(1+\chi_{i}\right)}{\epsilon}\right|^{2} \operatorname{Im}\left(x_{e}\right)+\frac{2 T_{i}}{\omega \alpha^{2} T_{e}}\left|\frac{x_{e}}{\epsilon}\right|^{2} \operatorname{Im}\left(x_{i}\right)
$$

Using Eq.'s (91), (92) and (93), the ion-acoustic Thomson spectra can be predicted accurately under the condition of collisionless electrons for any value of $\mathrm{k}_{\mathrm{fl}} \lambda_{\mathrm{ii}}, \mathrm{ZT}_{\mathrm{e}} / \mathrm{T}_{\mathrm{i}}$, and $U_{d}$. This claim can first be verified in the ion-collisionless limit where the exact form of $S(k, \omega)$ is already known.

The transport coefficients in the collisionless Landau limit are calculated from the perturbed ion distribution function $\left(f_{1}\right)$ for ion-acoustic waves which is obtained from the linearized Vlasov equation (Eq. (35)),

$$
f_{1}(v)=\frac{Z e \phi_{1}}{m_{i}} \frac{\frac{\partial F_{o}}{\partial v}}{\left(v-\frac{\omega_{i a}}{k_{i a} v_{i}}\right)}
$$

Here $F_{O}=\left[n_{O} /\left(2 \pi v_{j}{ }^{2}\right)^{1 / 2}\right] \exp \left(-v^{2} / 2 v_{i}{ }^{2}\right)$ is the Maxwellian background ion distribution and $\omega_{\mathrm{ia}}$ is the complex ion-acoustic frequency. Because there are no collisions, the perturbed distribution function is one-dimensional in Eq. (94). With the collisionless perturbed ion distribution function and the definitions from Eq.'s (86) - (89), the perturbed plasma quantities $n_{1}, u_{1}, T, q_{1}$, and $\pi_{1}$ in the Landau limit are given by,

$$
n_{1}=\frac{\operatorname{Ze}_{1}}{m_{i}} \int \frac{\frac{\partial F_{O}}{\partial \nu}}{\left(v-\frac{\omega_{i a}}{k_{i a} v_{i}}\right)} d v=\frac{Z_{e} \phi_{1}}{m_{i}} n_{o}\left(1+x_{i} Z\left(x_{i}\right)\right)
$$




$$
\begin{gathered}
u_{1}=\frac{Z e \phi_{1}}{m_{i}} \frac{v_{i}}{n_{o}} \int v \frac{\frac{\partial F_{o}}{\partial v}}{\left(v-\frac{\omega_{i a}}{k_{i a} v_{i}}\right)} d v=\frac{Z e \phi_{1}}{m_{i}} v_{i} x_{i}\left(1+x_{i} Z\left(x_{i}\right)\right) \\
T_{1}=\frac{Z e \phi_{1}}{m_{i}} \frac{m_{i} v_{i}^{2}}{n_{O}} \int\left(v^{2}-1\right) \frac{\frac{\partial F_{o}}{\partial v}}{\left(v-\frac{\omega_{i a}}{k_{i a} v_{i}}\right)} d v=Z e \phi_{1} v_{i}^{2}\left(1+\left(x_{i}^{2}-1\right)\left(1+x Z\left(x_{i}(j)\right)\right)\right. \\
q_{1}=\frac{Z e \phi_{1}}{m_{i}} \frac{m_{i} v_{i}^{3}}{2} \int\left(v^{3}-3 v\right) \frac{\frac{\partial F_{o}}{\partial v}}{\left(v-\frac{\omega_{i a}}{k_{i a} v_{i}}\right)} d v=\frac{Z e \phi_{1} n_{i} v_{i}^{3}}{2} x_{i}\left(1+\left(x_{i}^{2}-3\right)\left(1+x Z\left(x_{i}\right)\right)\right) \\
\pi_{1}=0
\end{gathered}
$$

where $\mathrm{x}_{\mathrm{i}}=\omega_{\mathrm{ia}} / \mathrm{k}_{\mathrm{ia}} \mathrm{v}_{\mathrm{i}}$ is again the normalized frequency and the perturbed stress tensor $\pi_{1}$ is identically zero because the problem is one-dimensional. The complex ion-acoustic frequency $x_{i}=\omega_{i a} / k_{i a} v_{i}$ in the equations is calculated by solving for the complex roots of the dielectric function $\epsilon=1+\chi_{\mathrm{e}}+\chi_{\mathrm{i}}$ which is given exactly by, see Eq. (37),

$$
\epsilon=1+\chi_{e}+\chi_{i}=1+\alpha^{2}\left[1+x_{e} Z\left(x_{e}\right)\right]+\frac{Z T_{e}}{T_{i}} \alpha^{2}\left[1+x_{i} Z\left(x_{i}\right)\right]=0
$$

in the Landau limit. When calculating the ion transport coefficients, the electron Landau 
damping is neglected by setting $x_{e}=0$ and the roots of the dielectric function are found in the quasineutral $\alpha \rightarrow \infty$ limit by,

$$
1+\frac{Z T_{e}}{T_{i}}\left[1+x_{i} Z\left(x_{i}\right)\right]=0
$$

There are two complex roots to this equation for a given $\mathrm{ZT}_{\mathrm{e}} / \mathrm{T}_{\mathrm{i}}$ ratio, $\mathrm{x}_{\mathrm{i}}=\left( \pm \omega_{\mathrm{ia}}\right.$ $\left.\mathrm{i} \gamma_{\mathrm{ia}}\right) / \mathrm{k}_{\mathrm{ia}} \mathrm{v}_{\mathrm{i}}$, where $\omega_{\mathrm{ia}} \neq 0$ is the ion-acoustic frequency and $\gamma_{\mathrm{ia}}$ is the ion Landau damping. The transport coefficients car now be calculated in the quasineutral, ioncollisionless limit with the ion-acoustic root from Eq. (101), Eq.'s (96) - (99) and the modified closure relations in Eq. (80). The results for $\kappa_{1}$ and $\kappa_{2}$ are given in Table I for different $\mathrm{ZT}_{\mathrm{e}} / \mathrm{T}_{\mathrm{i}}$ ratios, and the coefficients $\mu_{1}$ and $\mu_{2}$ are equal to zero in the collisionless limit since the perturbed stress tensor is identically zero. The real and imaginary parts of the ion-acoustic frequency in the quasineutral, Landau limit as predicted by Eq. (101) are also in Table I. To test the accuracy of the modified fluid model in the collisionless limit, the ion susceptibility from the model, Eq. (91), is substituted in Eq. (100) for the exact expression in Landau limit and the roots of the dielectric function are recalculated with $\mathrm{x}_{\mathrm{e}}=0, \alpha \rightarrow \infty, \mu_{1}=\mu_{2}=0, \Gamma=3$, and the values of $\kappa_{1}$ and $\kappa_{2}$ from Table 1 . The results for the real and imaginary parts of the ionacoustic frequency predicted by the modified fluid model ion susceptibility are listed in Table I and agree exactly with the correct values in the Landau limit. Therefore, the modified fluid model based on the fluid equations correctly predicts ion Landau damping.

The accuracy of the modified fluic model for predicting the ion-acoustic spectrum in the collisionless limit can be investigated by using the dynamical form factor, Eq. (93), 
Table I: Conductivity coefficients $\left(\kappa_{1}, \kappa_{2}\right)$ in the quasineutral Landau limit.

Also, the ion-acoustic frequency from Landau theory and the modified fluid model.

\begin{tabular}{|c|c|c|c|c|c|c||}
\hline $\mathrm{ZT}_{\mathrm{e}} / \mathrm{T}_{\mathrm{i}}$ & $\kappa_{\mathrm{l}}$ & $\kappa_{2}$ & $\begin{array}{c}\omega_{\mathrm{r}} / \mathrm{kv}_{\mathrm{i}} \\
\text { Landau }\end{array}$ & $\begin{array}{c}\omega_{\mathrm{r}} / \mathrm{kv}_{\mathrm{i}} \\
3-\mathrm{Pole}\end{array}$ & $\begin{array}{c}\omega_{\mathrm{i}} / \mathrm{kv}_{\mathrm{i}} \\
\text { Landau }\end{array}$ & $\begin{array}{c}\omega_{\mathrm{i}} / \mathrm{kv}_{\mathrm{i}} \\
3-\mathrm{Pole}\end{array}$ \\
\hline \hline 4 & -0.8577 & 0.7998 & 2.83 & 2.83 & 0.307 & 0.307 \\
\hline 6 & -0.6938 & 0.5757 & 3.17 & 3.17 & 0.177 & 0.177 \\
\hline 8 & -0.5747 & 0.4078 & 3.47 & 3.47 & 0.103 & 0.103 \\
\hline 10 & -0.4796 & 0.2790 & 3.73 & 3.73 & 0.0583 & 0.0583 \\
\hline 12 & -0.4008 & 0.1822 & 3.97 & 3.97 & 0.0321 & 0.0321 \\
\hline 14 & -0.3354 & 0.1128 & 4.20 & 4.20 & 0.0170 & 0.0170 \\
\hline 16 & -0.2822 & 0.06609 & 4.42 & 4.42 & 0.00866 & 0.00866 \\
\hline 20 & -0.2061 & 0.01947 & 4.84 & 4.84 & 0.00201 & 0.00201 \\
\hline 24 & -0.1594 & 0.004881 & 5.23 & 5.23 & $4.14 \mathrm{e}-4$ & $4.14 \mathrm{e}-4$ \\
\hline 28 & -0.1299 & 0.001094 & 5.59 & 5.59 & $7.91 \mathrm{e}-5$ & $7.91 \mathrm{e}-5$ \\
\hline 32 & -0.1099 & $2.271 \mathrm{e}-4$ & 5.93 & 5.93 & $1.43 \mathrm{e}-5$ & $1.43 \mathrm{e}-5$ \\
\hline \hline
\end{tabular}

with the collisionless electron susceptibility and interchanging the ion susceptibility between the exact form $\chi_{i}=\left(\mathrm{ZT}_{\mathrm{e}} / \mathrm{T}_{\mathrm{i}}\right) \alpha^{2}\left[1+\mathrm{x}_{\mathrm{i}} \mathrm{Z}\left(\mathrm{x}_{\mathrm{i}}\right)\right]$ and the expression given by the modified fluid model in Eq. (91). The spectral distribution predicted by the dynamical form factor $S(k, \omega)$ using the two different forms of the ion susceptibility is shown in Figure 43 for an aluminum plasma with ionization/temperature ratio $\left(\mathrm{ZT}_{\mathrm{e}} / \mathrm{T}_{\mathrm{i}}=8\right)$, in the quasineutral limit $(\alpha * 1)$, and with a relative drift $\left(\mathrm{k}_{\mathrm{ia}} \cdot \mathrm{U}_{\mathrm{d}}\right) / \mathrm{k}_{\mathrm{ia}} \mathrm{v}_{\mathrm{e}}=0.02$. The dashed line in Figure 43 is the ion-acoustic spectrum predicted using the exact ion susceptibility and the solid line is the spectrum predicted when using the ion susceptibility from the modified fluid model with $\mu_{1}=\mu_{2}=0, \Gamma=3$, and $\kappa_{1}=-0.5747, \kappa_{2}=0.4078$ from 




Figure 43: The ion-acoustic spectrum calculated from collisionless theory (- -) and from the modified fluid model $(-)$ with $\mathrm{ZT}_{\mathrm{e}} / \mathrm{Ti}=8$ and $\left(\mathrm{k}_{\mathrm{ia}} \cdot \mathrm{U}_{\mathrm{d}}\right) / \mathrm{k}_{\mathrm{ia}} \mathrm{v}_{\mathrm{e}}=.02$.

Table I. The spectrum predicted from the modified fluid model perfectly reproduces the peak height asymmetry and shape of the resonant features of the exact collisionless theory near $\omega / k v_{i}= \pm 3.47$. The only discrepancies between the modified fluid model and the exact result occur away from the ion-acoustic resonances at $\omega / \mathbf{k v}_{\mathrm{i}}= \pm 3.47$. The feature at $\omega / \mathrm{kv}_{\mathrm{i}}=0$ predicted by the modified fluid model is not the entropy wave, which cannot supported by a collisionless plasma. Instead, the feature results from holding the transport coefficients constant as a function of frequency. The transport coefficients are frequency dependent and by using the values for the coefficients at the ion-acoustic frequency, only the predicted spectrum near and at the ion-acoustic resonance is quantitatively correct. 
Since the primary motivation of the model in this chapter is to predict the peak height asymmetry as a function of relative drift, reduced accuracy in the spectral features predicted away from the peaks is not a concern. In the ion-collisionless limit $\left(k_{\mathrm{fl}} \lambda_{\mathrm{ii}} \rightarrow\right.$ $\infty)$, the modified fluid model accurately reproduces the peak height asymmetry for a given relative drift $\left(U_{d}\right)$. The model can now be extended into the intermediate ioncollisional regime $\left(\mathrm{k}_{\mathrm{f}} \lambda_{\mathrm{ii}} \approx 1\right)$ by solving the Fokker-Planck equation for the perturbed ion distribution function $\left(f_{1}\right)$, and then using the distribution function to calculate the transport coefficients $\kappa_{1}, \kappa_{2}, \mu_{1}, \mu_{2}$ and the ratio of the specific heats $\Gamma$ as a function of $\mathrm{k}_{\mathrm{fl}} \lambda_{\mathrm{ii}}$ and $\mathrm{ZT}_{\mathrm{e}} / \mathrm{T}_{\mathrm{i}}$. Once these quantities are known, the ion-acoustic spectrum, again assuming collisionless electrons, can be calculated with Eq.'s (91), (92), and (93) as was done in the ion-collisionless case presented in this section.

The linearized Fokker-Planck equation has been used by Ono and Kulsrud ${ }^{150}$, and Randall ${ }^{151}$ to study the ion-ion collisional effects on ion-acoustic waves in the intermediate regime $\left(0.1 \leq \mathrm{k}_{\mathrm{ia}} \lambda_{\mathrm{ii}} \leq 10\right)$. Their calculations involved solving the linearized Fokker-Planck equation as an initial value problem for the perturbed ion distribution function of an ion-acoustic wave using an Alternating-Direction-Implicit (ADI) technique. Although this technique did provide the correct solution, absolute convergence was not optimum. Since the method involves an initial value problem, a fairly accurate guess is needed in advance for the unknown perturbed ion distribution function to start the calculation. Even with an accurate guess, averaging techniques where necessary to eliminate the backward wave and transients that developed over time in the distribution function. In addition to these drawbacks, this method of solution will only 
work for propagating waves, and therefore could not be used to obtain information about the stationary entropy wave. The technique used in this chapter will treat the linearized Fokker-Planck equation as an eigenvalue problem. The three normal modes of the plasma, the entropy wave and the forward and backward traveling ion-acoustic waves, appear as the three least damped eigenvalues in the solution. The perturbed ion distribution functions do not need to be approximated in advance and are simply the corresponding eigenvectors of the normal mode eigenvalues. The perturbed ion distribution functions can then be used to calculate the transport coefficients $\kappa_{1}, \kappa_{2}, \mu_{1}$, $\mu_{2}$ and the ratio of specific heats $\Gamma$.

The Boltzmann equation with a Fokker-Planck collision term describes the evolution of the distribution function $\left(F_{\alpha}(r, v, t)\right)$ for a given species $\alpha$ in a plasma. In a non-magnetic plasma this equation is given by, ${ }^{152}$

$$
\begin{aligned}
\frac{\partial F_{\alpha}}{\partial t}+\vec{v} \cdot \vec{\nabla}, F_{\alpha}-\frac{Z_{\alpha} e}{m_{\alpha}} \vec{E} \cdot \vec{\nabla}_{v} F_{\alpha}= & \sum_{\beta}-\Gamma_{\alpha \beta} \vec{\nabla}_{v} \cdot\left(F_{\alpha} \vec{\nabla}_{v} H_{\alpha \beta}-\frac{1}{2} \vec{\nabla}_{v} \cdot F_{\alpha} \vec{\nabla}_{v} \vec{\nabla}_{v} G_{\alpha \beta}\right)(102) \\
= & \sum_{\beta} C\left(F_{\alpha}, F_{\beta}\right)
\end{aligned}
$$

and is commonly referred to as the Fokker-Planck equation. $\mathrm{Z}_{\alpha}$ is the charge state of species $\alpha\left(\mathrm{Z}_{\mathrm{e}}=-1\right.$ for electrons), $\mathrm{m}_{\alpha}$ is the mass, and $\mathrm{E}$ is the electric field vector. The summation is carried out over all species $B$ that collide with species $\alpha . \mathrm{H}_{\alpha \beta}, \mathrm{G}_{\alpha \beta}$ are the Rosenbluth potentials ${ }^{153}$ and $\Gamma_{\alpha \beta}$ is the collision strength parameter of species $\alpha$ and B given by (Ref. 152), 


$$
\begin{gathered}
H_{\alpha \beta}(\vec{v})=\frac{m_{\alpha}+m_{\beta}}{m_{\beta}} \int d \vec{v}^{\prime} F_{\beta}\left(\vec{v}^{\prime}\right)\left|\vec{v}-\vec{v}^{\prime}\right|^{-1} \\
G_{\alpha \beta}(\vec{v})=\int d \vec{v}^{\prime} F_{\beta}\left(\vec{v}^{\prime}\right)\left|\vec{v}-\vec{v}^{\prime}\right| \\
\vec{\nabla}^{2} H_{\alpha \beta}=-\frac{4 \pi\left(m_{\alpha}+m_{\beta}\right) F_{\beta}}{m_{\beta}} \\
\vec{\nabla}^{2} G_{\alpha \beta}=\frac{2 m_{\beta} H_{\alpha \beta}}{\left(m_{\alpha}+m_{\beta}\right)} \\
\Gamma_{\alpha \beta}=4 \pi\left(\frac{Z_{\alpha \beta} Z_{\beta} e^{2}}{m_{\alpha}}\right)^{2} \ln \Lambda_{\alpha \beta}
\end{gathered}
$$

where $\ln \Lambda_{\alpha \beta}$ is the Coulomb logarithm for collisions between $\alpha$ and $B$ in Eq. (107).

Equation (102) can be linearized by making the following substitutions for electrostatic waves,

$$
\begin{gathered}
F_{\alpha}(\vec{r}, \vec{v}, t)=f_{O}^{\alpha}(\vec{v})+f_{1}^{\alpha}(\vec{v}) e^{i(k z-\omega t)} \\
\vec{E}=-\vec{\nabla} \phi \quad \text { where } \phi=\phi_{O}+\phi_{1} e^{i(k z-\omega t)}
\end{gathered}
$$

where $\mathrm{f}_{\mathrm{O}}{ }^{\alpha}=\mathrm{n}_{\alpha} \exp \left(-\mathrm{v}^{2} / 2 \mathrm{v}_{\alpha}{ }^{2}\right) /\left(2 \pi \mathrm{v}_{\alpha}\right)^{3 / 2}$ is assumed to be Maxwellian, the perturbations are assumed small $\left(f_{1} / f_{O} * 1, \phi_{1} / \phi_{O} * 1\right)$, and the thermal velocity $\left(v_{\alpha}\right)$ of species $\alpha$ is given by, $v_{\alpha}=\left(k_{B} T m_{\alpha}\right)^{1 / 2}$. The perturbations are written with wave vectors in the $z$ direction so $f_{1}^{\alpha}(v)$ will possess azimuthal symmetry. The azimuthal symmetry of $f_{1}^{\alpha}(v)$ coupled with the spherical symmetry of $f_{0}{ }^{\alpha}(v)$ will simplify the expressions for the collision terms in the following section. The linearized Fokker-Planck equations for the ions and electrons are now written with the aid of Eq. (108) as, 


$$
\begin{gathered}
i\left(k v_{z}-\omega\right) f_{1}^{i}+i \frac{Z e \phi_{1} k v_{z}}{T_{i}} f_{o}^{i}=C\left(f_{o}^{i}, f_{1}^{i}\right)+C\left(f_{1}^{i}, f_{o}^{i}\right) \\
i\left(k v_{z}-\omega\right) f_{1}^{e}-i \frac{e \phi_{1} k v_{z}}{T_{c}} f_{o}^{e}=0
\end{gathered}
$$

where all terms are first order in the perturbation. Equations (109) and (110) have been written under the assumption that the electrons are collisionless $\left(\mathrm{k}_{\mathrm{fl}} \lambda_{\mathrm{ei}}, \mathrm{k}_{\mathrm{fl}} \lambda_{\mathrm{ee}}\right.$ "1). The perturbed potential $\phi_{1}$ is found by solving Eq. (110) for the perturbed electron density $\mathrm{n}_{1}{ }^{\mathrm{e}}$ using the standard Landau prescription (Ref. 121),

$$
n_{1}^{e}=\int f_{1}^{e} d \vec{v}+\frac{e \phi_{1}}{T_{e}} \int \frac{k v f_{o}^{e}}{\left(k v_{z}-\omega\right)} d \vec{v}=\frac{e n_{O}^{e}}{T_{e}} \phi_{1}\left[1+\left(\frac{\omega}{k v_{e}}\right) \chi\left(\frac{\omega}{k v_{e}}\right)\right]
$$

where $\mathrm{Z}$ is the plasma dispersion function defined in Eq. (37). The solution for $\phi_{1}$ in the limit $\left(\omega / k_{\mathrm{e}} \rightarrow 0\right)$ is,

$$
\phi_{1}=\frac{T_{e} n_{1}^{e}}{e n_{o}^{e}} \approx \frac{T_{e} n_{1}^{i}}{e n_{O}^{i}}
$$

where quasineutrality $(\alpha \rightarrow \infty)$ has been assumed for the approximation in terms of the ion density $n_{O}{ }^{i}$ and perturbed ion density $n_{1}{ }^{i}=\int f_{1}{ }^{i} d^{3} v$. By taking the limit $\left(\omega / k v_{e} \rightarrow\right.$ $0)$, electron Landau damping is ignored. Since the electrons and ions are decoupled $\left(k \lambda_{e i}\right.$ " 1), the electron Landau damping can be included afterwards by adding $\left(\pi \mathrm{Zm}_{\mathrm{e}} / 8 \mathrm{~m}_{\mathrm{i}}\right)^{1 / 2} \operatorname{Re}\left(\omega_{\mathrm{ia}} / \mathrm{k}_{\mathrm{ia}} \mathrm{v}_{\mathrm{i}}\right)$ where $\omega_{\mathrm{ia}}$ is the complex ion-acoustic wave frequency. Note, that the effects of electron-ion collisions, $\mathrm{k} \lambda_{\mathrm{ei}} \approx 1$, on the electron damping of ionacoustic waves has been recently calculated by Epperlein ${ }^{154}$ et al. 
The collision terms in Eq. (109) can be simplified by working in spherical coordinates: $\mathbf{v}=|\mathbf{v}| / \mathbf{v}_{\mathrm{i}}, \mu=\mathbf{v}_{\mathrm{z}} / \mathbf{v}$, and $\phi$, and by utilizing the spherical symmctry of $f_{O}^{i}(v)$ and the azimuthal symmetry of $f_{1}^{i}(v)$,

$$
\begin{gathered}
C\left(f_{o}, f_{1}\right)=\Gamma_{u}\left[4 \pi f_{\partial} f_{1}-\frac{H_{O l}}{2 v_{i}^{3}} f_{O}+\frac{v^{2}}{2 v_{i}^{3}} \frac{\partial^{2} G_{O l}}{\partial v^{2}} f_{o}\right] \\
C\left(f_{1}, f_{O}\right)=\frac{-\Gamma_{u}}{v^{2} v_{i}^{3}} \frac{\partial}{\partial v}\left[\frac{v}{2} \frac{\partial H_{1 O}}{\partial v} e^{-v^{2} / 2} \frac{\partial}{\partial v}\left(e^{v^{2} / 2} f_{1}\right)\right]+\frac{\Gamma_{u}}{2 v^{3} v_{i}^{3}} \frac{\partial G_{1 o}}{\partial v}\left(\frac{\partial}{\partial \mu}\left(1-\mu^{2}\right) \frac{\partial f_{1}}{\partial \mu}\right)
\end{gathered}
$$

The ion superscripts are inferred in Eq. (113) where $\left(\mathrm{H}_{\mathrm{O} 1}, \mathrm{G}_{\mathrm{O} 1}\right)$ and $\left(\mathrm{H}_{10}, \mathrm{G}_{10}\right)$ refer to the Rosenbluth potentials of the perturbed $f_{1}$ and background Maxwellian $f_{O}$ ion distribution functions, respectively. The collision strength parameter $\Gamma_{\mathrm{ii}}$ and the Maxwellian Rosenbluth potentials $\mathrm{H}_{10}$ and $\mathrm{G}_{10}$ are given by (Ref. 152),

$$
\begin{gathered}
\Gamma_{i i}=\frac{4 \pi Z^{4} e^{4}}{m_{i}^{2}} \ln \Lambda_{i i}=\frac{3 \sqrt{\pi} v_{i}^{4}}{\lambda_{i i} n_{O}} \\
\frac{\partial H_{1 O}}{\partial \nu}=\frac{2\left(\sqrt{\frac{2}{\pi}} v e^{-v^{2} / 2}-E r f\left(\frac{v}{\sqrt{2}}\right)\right)}{v^{2}} \\
\frac{\partial G_{1 O}}{\partial \nu}=\operatorname{Erf}\left(\frac{v}{\sqrt{2}}\right)+\frac{1}{2} \frac{\partial H_{1 O}}{\partial \nu}
\end{gathered}
$$

where $\operatorname{Erf}(x)$ is the error function.

The perturbed ion distribution function $\mathrm{f}_{1}$ can now be written as an expansion in Legendre polynomials $P_{L}$, 


$$
f_{1}(v, \mu)=\sum_{L=0}^{\infty} a_{L}(v) P_{L}(\mu)
$$

By making the normalizations, $f_{O} \rightarrow\left(n_{O} /\left(2 \pi v_{i}^{2}\right)^{3 / 2}\right) f_{O}$ and $f_{1} \rightarrow\left(n_{O} /\left(2 \pi v_{i}^{2}\right)^{3 / 2}\right) f_{1}$, equations (109), (112), (113), and (114) can be combined into a series of coupled equations of the coefficient $a_{L}(v)$ in the following form,

$$
\begin{gathered}
\left(\frac{L v a_{L-1}}{2(L-1)+1}+\frac{(L+1) v a_{L+1}}{2(L+1)+1}\right)+\left(\frac{Z T_{e}}{T_{i}} \frac{4 \pi v f_{O} \int_{0}^{\infty} a_{o}(v) v^{2} d v}{(2 \pi)^{3 / 2}}\right) \delta_{L I}+ \\
i\left(\frac{3 \sqrt{2} f_{\sigma_{L}} a_{L}}{k \lambda_{i i}}-\frac{3 f_{O}}{2^{3 / 2} \pi k \lambda_{i i}}\left(\frac{H_{L}}{2}-\frac{v^{2}}{2} \frac{\partial^{2} G_{L}}{\partial v^{2}}\right)\right)+ \\
i\left(\frac{-3 \sqrt{\pi}}{v^{2} k \lambda_{i i}}\left(\frac{\partial}{\partial v}\left[\frac{v}{2} \frac{\partial H_{1 O}}{\partial v} e^{-v^{2} / 2} \frac{\partial}{\partial v}\left(e^{v^{2} / 2} a_{L}(v)\right)\right]-\frac{1}{2 v} \frac{\partial G_{1 O}}{\partial v} L(L+1) a_{L}\right)\right)=\omega a_{L}
\end{gathered}
$$

where $\delta_{\mathrm{L} 1}$ is the Kronecker delta and the Legendre term Rosenbluth potentials $\mathrm{H}_{\mathrm{L}}$ and $\partial^{2} G_{L} / \partial v^{2}$ are given by (Ref. 153),

$$
\begin{gathered}
H_{L}(v)=\frac{8 \pi}{(2 L+1) v}\left[\frac{1}{v^{L}} \int_{0}^{v} a_{L}\left(v^{\prime}\right) v^{\prime 2+L} d v^{\prime}+v^{L+1} \int_{v}^{\infty} a_{L}\left(v^{\prime}\right) v^{\prime 1-L} d v^{\prime}\right] \\
\frac{\partial^{2} G_{L}(v)}{\partial v^{2}}=\frac{-4 \pi}{\left(4 L^{2}-1\right)} \int_{0}^{v} a_{L^{\prime}}\left(v^{\prime}\right) v^{\prime 2+L}\left(\frac{L(L-1)}{v^{L+1}}-\frac{(L+1)(L+2)(L-1 / 2) v^{\prime 2}}{(L+3 / 2) v^{L+3}}\right) d v^{\prime}- \\
\frac{4 \pi}{\left(4 L^{2}-1\right)} \int_{v}^{\infty} \frac{a_{L}\left(v^{\prime}\right)}{v^{\prime L-3}}\left(L(L-1) v^{L-2}-\frac{(L+1)(L+2)(L-1 / 2) v^{L}}{(L+3 / 2) v^{\prime 2}}\right) d v^{\prime}
\end{gathered}
$$

Equations (116) and (117) can be expressed in the form of an eigenvalue problem by finite differencing the derivative terms and discretizing the integrals on a velocity grid $v_{j}$ $=\left\{v_{1}, v_{2}, \ldots, v_{j \max }\right\}=\left\{v \max / j \max , 2^{*} v \max / j \max , \ldots, v \operatorname{vmax}\right\}$, where vmax is the maximum velocity used and jmax is the number of grid points. A grid point at $\mathbf{v}=0$ is 
not used to avoid divergence of Eq. (116) as $v \rightarrow 0$. The general eigenvalue problem now takes the form,

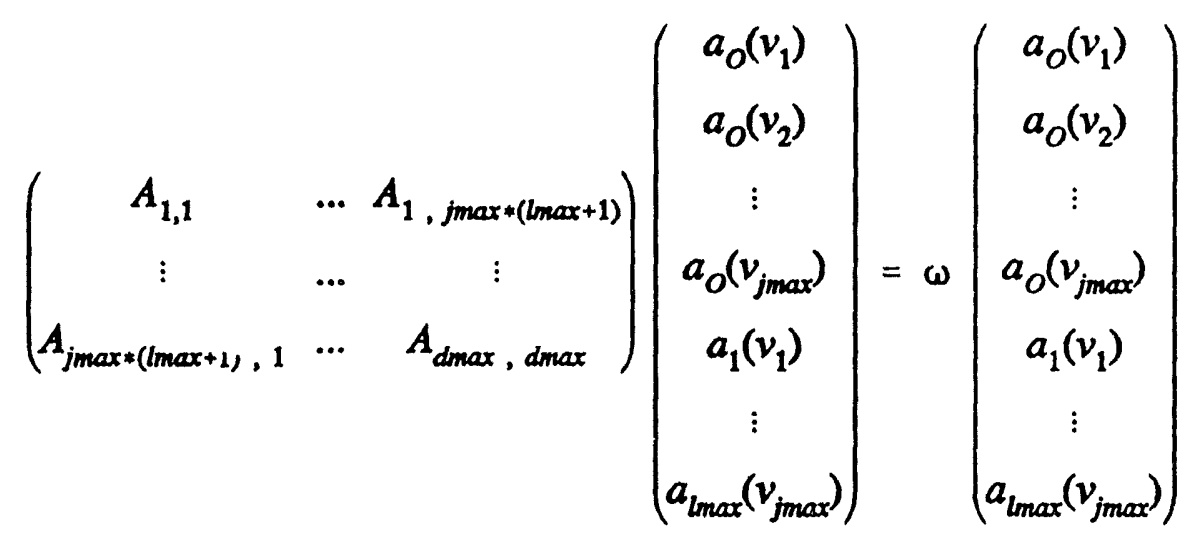

where Imax is the maximum number of Legendre polynomials considered in the problem. The dimension of the matrix $(\operatorname{dmax}=j \max *(\max +1))$ is determined by the product of the number of velocity grid points and Legendre polynomials used.

The source code for the program entitled 'FOKKER.FOR' that solves Eq. (118) is listed in the APPENDIX. The solution of Eq. (118) yields dmax eigenvalues of which three are least damped, i.e. smallest imaginary part: two have real frequencies at plus and minus the phase velocity of the ion-acoustic wave and correspond to the forward and backward traveling waves, respectively, and one has zero real frequency which corresponds to the stationary entropy or zero frequency wave. Once the eigenvalues for these three modes have been determined, the eigenvectors, perturbed distribution functions $f_{1}$, can be found and then used to calculate the transport coefficients $\kappa_{1}, \kappa_{2}$, $\mu_{1}, \mu_{2}$ and the ratio of specific heats $\Gamma$ for the plasma conditions of interest. The accuracy of the eigenvalue method depends on the number of grid points (jmax), the maximum velocity (vmax), and the total number of Legendre modes $(\operatorname{lmax}+1)$. The 
values of the quantities jmax, vmax, and Imax needed for convergence to a desired level of accuracy depend on the plasma parameters $\mathrm{ZT}_{\mathrm{e}} / \mathrm{T}_{\mathrm{i}}$ and $\mathrm{k} \lambda_{\mathrm{ii}}$ and this dependence will be discussed in the following sections.

Table II: The damping coefficients $\gamma_{\mathrm{ia}}{ }^{\mathrm{o}}$ and $\gamma_{\mathrm{ent}}{ }^{\mathrm{o}}$ for the ion-acoustic and entropy waves in the collisional limit.

\begin{tabular}{||l|l|l|}
\hline \hline $\mathrm{ZT}_{\mathrm{e}} / \mathrm{T}_{\mathrm{i}}$ & ${\gamma_{\mathrm{ia}}{ }^{\circ}}$ & $\gamma_{\mathrm{ent}}{ }^{\circ}$ \\
\hline 1 & 0.9650 & 0.5000 \\
\hline 2 & 0.8763 & 0.5453 \\
\hline 4 & 0.7929 & 0.5882 \\
\hline 8 & 0.7297 & 0.6207 \\
\hline 16 & 0.6891 & 0.6418 \\
\hline 32 & 0.6657 & 0.6531 \\
\hline 48 & 0.6575 & 0.6576 \\
\hline 64 & 0.6532 & 0.6599 \\
\hline
\end{tabular}

To guarantee that the results from the Fokker-Planck calculations approach the proper collisional and collisionless limits, a brief overview of the complex frequency of the entropy and ion-acoustic waves in the two limits will be discussed for comparison with the calculations. The complex frequencies for the normal modes of the plasma are found from the roots of the dielectric function $(\epsilon=0)$. This has already been done for the quasineutral, collisionless limit ignoring electron Landau damping in Eq. (101), where only ion-acoustic waves are supported by the plasma. The complex frequency of the ionacoustic wave for certain $\mathrm{ZT}_{\mathrm{e}} / \mathrm{T}_{\mathrm{i}}$ values is listed in Table I. The dielectric function $(\epsilon=$ 
$\left.1+x_{\mathrm{e}}+x_{\mathrm{i}}\right)$ can be expressed in the quasineutral $(\alpha \rightarrow \infty)$, collisional limit by again ignoring the effects of the electrons $\left(\chi_{\mathrm{e}}=\alpha^{2}\right)$ and using the fluid limit expression for the ion susceptibility, Eq. (44) from Chapter 2. The equation $\epsilon=0$ for the quasineutral, ioncollisional limit is given by,

$$
1-\frac{Z T_{e}}{T_{i}} \frac{x_{i}+\frac{2}{3} i \kappa^{\circ}}{x_{i}^{3}+\frac{2}{3} i\left(2 \mu^{o}+\kappa^{o}\right) x_{i}^{2}-\frac{1}{3}\left(5+\frac{8}{3} \mu^{\circ} \kappa^{o}\right) x_{i}-\frac{2}{3} i \kappa^{o}}=0
$$

There are three complex roots to this equation for a given $\mathrm{ZT}_{\mathrm{e}} / \mathrm{T}_{\mathrm{i}}$ ratio. Two roots are $\mathrm{x}_{\mathrm{i}}=\left( \pm \omega_{\mathrm{ia}}-\mathrm{i} \gamma_{\mathrm{ia}}\right) / \mathrm{k}_{\mathrm{ia}} \mathrm{v}_{\mathrm{i}}$, where $\omega_{\mathrm{ia}} / \mathrm{k}_{\mathrm{ia}} \mathrm{v}_{\mathrm{i}}=\left(\left(\mathrm{ZT}_{\mathrm{e}} / \mathrm{T}_{\mathrm{i}}\right)+(5 / 3)\right)^{1 / 2}$ is the ion-acoustic frequency and $\gamma_{i a}$ is the collisional damping of the wave, and the third root represents the entropy wave, where $x_{i}=\left(\omega_{\text {ent }}-i \gamma_{\text {ent }}\right) / \mathrm{k}_{\text {ent }} v_{i}, \omega_{\text {ent }}=0$ and $\gamma_{\text {ent }}$ is the damping of the wave. Using Eq. (119), it can be shown that the collisional damping of the ionacoustic waves is proportional to $\mathrm{k}_{\mathrm{ia}} \lambda_{\mathrm{ii}}$ and is given by, $\gamma_{\mathrm{ia}}=\gamma_{\mathrm{ia}}{ }^{\circ} \mathrm{k}_{\mathrm{ia}} \lambda_{\mathrm{ii}}$. The damping of the entropy wave is proportional to $\mathrm{k}_{\mathrm{ent}} \lambda_{\mathrm{ii}}$ with $\gamma_{\mathrm{ent}}=\gamma_{\mathrm{ent}}{ }^{\circ} \mathrm{k}^{\mathrm{o}}=3.9 \gamma_{\mathrm{ent}}{ }^{\mathrm{o}} \mathrm{k}_{\mathrm{ent}} \lambda_{\mathrm{ii}}$. The damping coefficients $\gamma_{\mathrm{ia}}{ }^{\mathrm{o}}$ and $\gamma_{\mathrm{ent}}{ }^{\mathrm{o}}$ are a function of the ratio $\mathrm{ZT} \mathrm{e}_{\mathrm{e}} / \mathrm{T}_{\mathrm{i}}$ and the values of the damping coefficients determined from Eq. (119) are listed in Table II for a few representative $\mathrm{ZT}_{\mathrm{e}} / \mathrm{T}_{\mathrm{i}}$ ratios.

Although the primary motivation of this chapter is to develop a model for the peak height asymmetry in the ion-acoustic Thomson spectrum in the intermediate collisional regime, the new entropy wave results from the Fokker-Planck calculation are also significant and deserve some discussion. The presence of an entropy wave in a plasma has been studied to a limited extent by recent Thomson scattering experiments in 
Table III: Damping and ion thermal conductivity for the entropy wave as a function of $k_{e n t} \lambda_{i i}$ for $2 T_{e} / T_{i}$ ratios of 2,4 , and 8 .

\begin{tabular}{|c|c|c|c|c|c|c|}
\hline $\mathrm{k}_{\mathrm{ent}} \lambda_{\mathrm{ii}}$ & $\begin{array}{l}\gamma_{\text {ent }} / k_{\mathrm{i}} \\
\mathrm{ZT}_{\mathrm{e}} / \mathrm{T}_{\mathrm{i}} \\
\quad=2\end{array}$ & $\begin{array}{l}\gamma_{\text {ent }} / \mathrm{kv}_{\mathrm{i}} \\
\mathrm{ZT}_{\mathrm{e}} / \mathrm{T}_{\mathrm{i}} \\
=4\end{array}$ & $\begin{array}{l}\gamma_{\text {ent }} / k v_{i} \\
\mathrm{ZT}_{\mathrm{e}} / \mathrm{T}_{\mathrm{i}} \\
\quad=8\end{array}$ & $\begin{array}{l}\mathrm{k}_{2} / \mathrm{k} \lambda_{\mathrm{ii}} \\
\mathrm{ZT}_{\mathrm{e}} / \mathrm{T}_{\mathrm{i}} \\
=2\end{array}$ & $\begin{array}{l}\kappa_{2} / k \lambda_{\mathrm{ii}} \\
\mathrm{ZT}_{\mathrm{e}} / \mathrm{T}_{\mathrm{i}} \\
=4\end{array}$ & $\begin{array}{r}\kappa_{2} / k \lambda_{\mathrm{ii}} \\
\mathrm{ZT}_{\mathrm{e}} / \mathrm{T}_{\mathrm{i}} \\
=8\end{array}$ \\
\hline 0.034 & 0.0702 & 0.0758 & 0.0800 & 3.87 & 3.87 & 3.88 \\
\hline 0.067 & 0.133 & 0.146 & 0.151 & 3.71 & 3.71 & 3.72 \\
\hline 0.133 & 0.232 & 0.253 & 0.263 & 3.32 & 3.34 & 3.34 \\
\hline 0.266 & 0.370 & 0.395 & 0.411 & 2.75 & 2.78 & 2.80 \\
\hline 0.398 & 0.459 & 0.487 & 0.504 & 2.39 & 2.44 & 2.47 \\
\hline 0.532 & 0.526 & 0.552 & 0.569 & 2.14 & 2.20 & 2.24 \\
\hline 0.798 & 0.620 & 0.645 & 0.660 & 1.83 & 1.91 & 1.96 \\
\hline 1.064 & 0.688 & 0.708 & 0.721 & 1.64 & 1.74 & 1.81 \\
\hline 1.33 & 0.740 & 0.760 & 0.772 & 1.51 & 1.61 & 1.69 \\
\hline
\end{tabular}

moderate-density $\left(\mathrm{n}_{\mathrm{e}} \approx 1 \times 10^{17} \mathrm{~cm}^{-3}\right)$, low ionization/temperature ratio $\left(\mathrm{ZT}_{\mathrm{e}} / \mathrm{T}_{\mathrm{i}} \approx 1\right)$, highly collisional plasmas (Ref. 127). The entropy wave forms from thermal level ion temperature fluctuations in the plasma which are balanced by ion density fluctuations to produce zero pressure. Since there are no pressure gradients, the wave is stationary and has zero mode frequency. Because the entropy wave is driven by ion temperature fluctuations, the damping of the wave is primarily due to the ion thermal conductivity of the plasma. The entropy wave has a comparable damping rate to a corresponding ionacoustic wave of the same wave number in the collisional $\left(\mathrm{k}_{\mathrm{f} 1} \lambda_{\mathrm{ii}} \rightarrow 0\right)$ limit, but in the collisionless (Landau) limit $\left(\mathrm{k}_{\mathrm{fl}} \lambda_{\mathrm{ii}} \rightarrow \infty\right)$, propagating ion-acoustic waves are present with finite damping, while the entropy wave cannot be supported. The theory developed prior 




Figure 44: Reduction in the damping of the entropy wave from collisional 'Braginskii' theory when $\mathrm{k}_{\text {ent }} \lambda_{\mathrm{ii}} \approx 1$ for a ratio of $\mathrm{ZT}_{\mathrm{e}} / \mathrm{T}_{\mathrm{i}}=4$.

to the work presented in this thesis is based on the Braginskii transport equations and is strictly valid only in the collisional or fluid limit (Ref. 127). The fluid theory breaks down in the region of $\mathrm{k}_{\mathrm{fl}} \lambda_{\mathrm{ii}} \approx 1$, because the Braginskii transport coefficients are modified due to finite collisional effects. Specifically, the damping of the entropy wave will be modified from the fluid result in the region of $\mathrm{k}_{\mathrm{f} 1} \lambda_{\mathrm{ii}} \approx 1$ due to a reduction in the ion thermal conductivity. The Fokker-Planck equation must be used to study this phenomenon. Utilizing the eigenvalue approach to solving the Fokker-Planck equation, the damping of the entropy wave is found as a function of $\mathrm{k}_{\mathrm{ent}} \lambda_{\mathrm{ii}}$ for $\mathrm{ZT}_{\mathrm{e}} / \mathrm{T}_{\mathrm{i}}$ ratios of 2 , 4 , and 8 where $k_{\text {ent }}$ is the wave number of the entropy wave. The numerical results are 




Figure 45: Reduction in the ion thermal conductivity $\kappa_{2}$ for the entropy wave from the collisional 'Braginskii' limit when $k_{\text {ent }} \lambda_{i i} \approx 1$ for a ratio of $\mathrm{ZT}_{\mathrm{e}} / \mathrm{T}_{\mathrm{i}}=4$.

listed in Table III and displayed graphically in Figure 44 for $\mathrm{ZT}_{\mathrm{e}} / \mathrm{T}_{\mathrm{i}}=4$. The damping rate predicted by collisional theory, Eq. (119), is included in Figure 44 for comparison. As shown by the figure, the computed damping of the entropy wave is equivalent to the collisional theory of Eq. (119) for small $\mathrm{k}_{\text {ent }} \lambda_{\mathrm{ii}}$ and approaches an infinite value as $\mathrm{k}_{\mathrm{ent}} \lambda_{\mathrm{ii}}$ $\rightarrow \infty$, but at a reduced rate from the collisional prediction. Since the electrons are assumed collisionless, the damping of the entropy wave is due solely to the ion thermal conductivity, and a reduction from the collisional damping rate implies the ion thermal conductivity constant must decrease from the Braginskii value of 3.9 as $\mathrm{k}_{\mathrm{ent}} \lambda_{\mathrm{ii}}$ increases. The transport coefficients $\kappa_{1}, \kappa_{2}, \mu_{1}, \mu_{2}$ can be calculated from the perturbed distribution 
functions for the entropy wave using the modified closure relations (Eq. (80)) and Eq.'s (85) - (89). It is found that only the transport coefficient $\kappa_{2}$ is non-zero independent of the value of $\mathrm{ZT}_{\mathrm{e}} / \mathrm{T}_{\mathrm{i}}$ and $\mathrm{k}_{\text {ent }} \lambda_{\mathrm{ii}}$. In addition, the viscosity coefficients $\mu_{1}$ and $\mu_{2}$ are always zero for the entropy wave, since the wave does not propagate. Because $k_{2}$ multiplies the $\nabla \mathrm{T}$ term in the closure relation for the heat flux (q), it can be directly associated with the ion thermal conductivity $\left(k_{i}\right)$. The computed ratio $k_{2} / k_{\text {ent }} \lambda_{i i}$ is given in Table III for the $\mathrm{ZT}_{\mathrm{e}} / \mathrm{T}_{\mathrm{i}}$ ratios 2,4 , and 8 and shown graphically in Figure 45 for $\mathrm{ZT}_{\mathrm{e}} / \mathrm{T}_{\mathrm{i}}=4$. As shown by the table and the figure, the ion thermal conductivity for the entropy wave does indeed decrease from the Braginskii limit of 3.9 for small $k_{\text {ent }} \lambda_{i i}$ as $\mathrm{k}_{\mathrm{ent}} \lambda_{\mathrm{ii}}$ increases.

The calculations for the entropy wave are currently limited to values of $\mathrm{k}_{\mathrm{ent}} \lambda_{\mathrm{ii}}<$ 1.33 because of the number of Legendre modes required for convergence of the computed damping at larger values of $\mathrm{k}_{\mathrm{ent}} \lambda_{\mathrm{ii}}$. A three significant figure accuracy is obtained for the damping of the entropy wave for $k_{\text {ent }} \lambda_{i i}$ values of 0.034 and 0.067 with only 3 Legendre modes while for $\mathrm{k}_{\text {ent }} \lambda_{\mathrm{ii}}=1.33,10$ Legendre modes are needed for the desired accuracy. As $\mathrm{k}_{\mathrm{ent}} \lambda_{\mathrm{ii}} \rightarrow \infty$, an infinite number of Legendre modes would be needed to compute the correct answer. The requirement of a large number of Legendre modes in the collisionless limit was similarly found by Epperlein et al. (Ref. 154) when considering electron-ion collisional effects on ion-acoustic waves. Since a three significant figure accuracy requires a velocity grid of $\approx 100$ points on the interval $\mathrm{v} / \mathrm{v}_{\mathrm{i}}=\{0,7\}$, the damping at $\mathrm{k}_{\text {ent }} \lambda_{\mathrm{ii}}=$ 1.33 is derived from the eigenvalues of a $1000 \times 1000$ complex matrix. The damping of the entropy wave at higher values of $\mathrm{k}_{\mathrm{ent}} \lambda_{\mathrm{ii}}$ would require the eigenvalues of even larger 
matrices which is time prohibitive on the computers being used for these calculations.

Table IV: Eigenvalue results for the complex ion-acoustic frequency with $\mathrm{ZT}_{\mathrm{e}} / \mathrm{T}_{\mathrm{i}}=8$. (ADI) results from Ref. 151 are included for comparison.

\begin{tabular}{|l|l|l|l|l||}
\hline \hline$k_{\mathrm{ia}} \lambda_{\mathrm{ii}}$ & $\begin{array}{l}\omega_{\mathrm{ia}} / \mathrm{k}_{\mathrm{ia}} \mathrm{v}_{\mathrm{i}} \\
\text { (Eigenvalue) }\end{array}$ & $\begin{array}{l}\gamma_{\mathrm{ia}} / \mathrm{k}_{\mathrm{ia}} \mathrm{v}_{\mathrm{i}} \\
\text { (Eigenvalue) }\end{array}$ & $\begin{array}{l}\omega_{\mathrm{ia}} / \mathrm{k}_{\mathrm{ia}} \mathrm{v}_{\mathrm{i}} \\
(\mathrm{ADI})\end{array}$ & $\begin{array}{l}\gamma_{\mathrm{ia}} / \mathrm{k}_{\mathrm{ia}} \mathrm{v}_{\mathrm{i}} \\
(\mathrm{ADI})\end{array}$ \\
\hline 0.034 & 3.111 & 0.0247 & $\mathrm{~N} / \mathrm{A}$ & $\mathrm{N} / \mathrm{A}$ \\
\hline 0.067 & 3.116 & 0.0474 & $\mathrm{~N} / \mathrm{A}$ & $\mathrm{N} / \mathrm{A}$ \\
\hline 0.133 & 3.133 & 0.0866 & 3.13 & 0.084 \\
\hline 0.266 & 3.179 & 0.140 & 3.18 & 0.139 \\
\hline 0.398 & 3.225 & 0.169 & 3.22 & 0.168 \\
\hline 0.532 & 3.264 & 0.181 & 3.26 & 1.181 \\
\hline 0.798 & 3.319 & 0.185 & 3.31 & 0.185 \\
\hline 1.064 & 3.353 & 0.179 & 3.35 & 0.179 \\
\hline 1.33 & 3.375 & 0.172 & 3.37 & 0.172 \\
\hline 1.597 & 3.391 & 0.166 & 3.39 & 0.166 \\
\hline 2.128 & 3.410 & 0.155 & 3.41 & 0.155 \\
\hline 2.66 & 3.422 & 0.147 & 3.42 & 0.146 \\
\hline 3.192 & 3.430 & 0.141 & 3.43 & 0.141 \\
\hline 3.724 & 3.436 & 0.137 & 3.43 & 0.136 \\
\hline 5.32 & 3.445 & 0.128 & 3.44 & 0.127 \\
\hline 10.64 & 3.456 & 0.116 & 3.46 & 0.115 \\
\hline 21.28 & 3.461 & 0.109 & 3.47 & 0.103 \\
\hline 42.56 & 3.464 & 0.106 & $\mathrm{~N} / \mathrm{A}$ & $\mathrm{N} / \mathrm{A}$ \\
\hline 85.12 & 3.465 & 0.104 & $\mathrm{~N} / \mathrm{A}$ & $\mathrm{N} / \mathrm{A}$ \\
\hline & & & & \\
\hline
\end{tabular}

The complex frequencies and perturbed distribution functions are calculated as a 




Figure 46: Damping of the ion-acoustic wave found from the solution of the FokkerPlanck equation for $\mathrm{ZT}_{\mathrm{e}} / \mathrm{T}_{\mathrm{i}}=8$. Collisional 'Braginskii' theory and the collisionless 'Landau' limit are included.

function of $\mathrm{k}_{\mathrm{ia}} \lambda_{\mathrm{ii}}$ for ratio $\mathrm{ZT}_{\mathrm{e}} / \mathrm{T}_{\mathrm{i}}=8$ for the ion-acoustic wave. The ionization/temperature ratio of 8 is chosen because a portion of the laser plasma diagnosed in Chapter 4 lies in this parameter regime. The complex frequencies for the ion-acoustic wave are listed in Table IV and the damping values are shown graphically in Figure 46. The damping rates predicted from collisional theory, Eq. (119), and collisionless theory, Table I, are shown in the figure for comparison. As shown by the figure, the computed damping of the ion-acoustic wave is equivalent to the collisional theory for small $k_{\mathrm{ia}} \lambda_{\mathrm{ii}}$ and approaches the collisionless Landau limit for $\mathrm{k}_{\mathrm{ia}} \lambda_{\mathrm{ii}} \rightarrow \infty$. Also included in Table IV are the complex frequencies computed for the same $\mathrm{ZT}_{\mathrm{e}} / \mathrm{T}_{\mathrm{i}}=8$ 
ratio from Reference 151 using the (ADl) technique. For the common values of $\mathrm{k}_{\mathrm{ia}} \lambda_{\mathrm{ii}}$, the real and imaginary parts of the complex frequency from the eigenvalue solution of the Fokker-Planck equation and the (ADI) results of Ref. 151 agree to the third significant figure. A precision of three significant figures is reasonable for these calculations because experimental verification of the theory cannot typically be done to better than $1 \%$ accuracy.

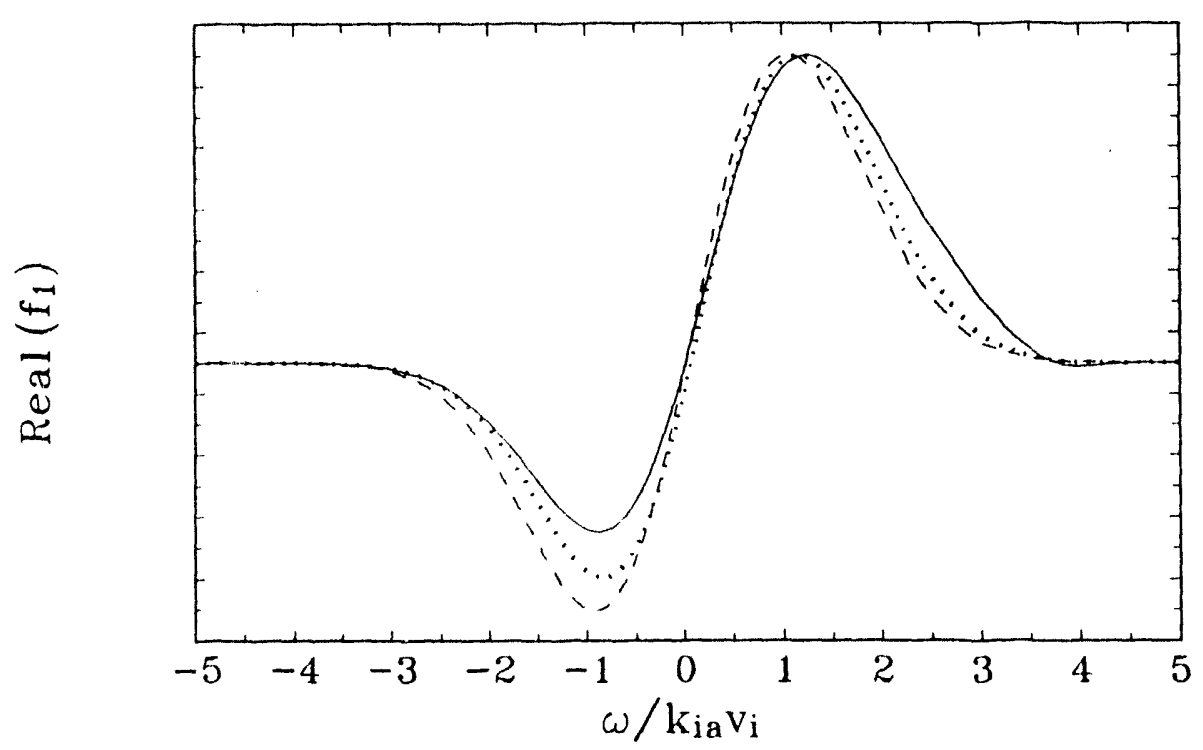

Figure 47: Phase-amplitude normalized real part of the perturbed distribution function for the ion-acoustic wave with $\mathrm{ZT}_{\mathrm{e}} / \mathrm{T}_{\mathrm{i}}=8$ and $\mathrm{k}_{\mathrm{ia}} \lambda_{\mathrm{ii}}=0.133$ (dashes), 0.532 (dots), and 2.66 (line).

To calculate the peak height asymmetry in the ion-acoustic Thomson spectra for a ratio $\mathrm{ZT}_{\mathrm{e}} / \mathrm{T}_{\mathrm{i}}=8$ given the collisionality $\left(\mathrm{k}_{\mathrm{ia}} \lambda_{\mathrm{ii}}\right)$ and the relative drift $\left(\mathrm{U}_{\mathrm{d}}\right)$, the collisionally dependent transport coefficients $\kappa_{1}, \kappa_{2}, \mu_{1}, \mu_{2}$ and the ratio of specific heats 


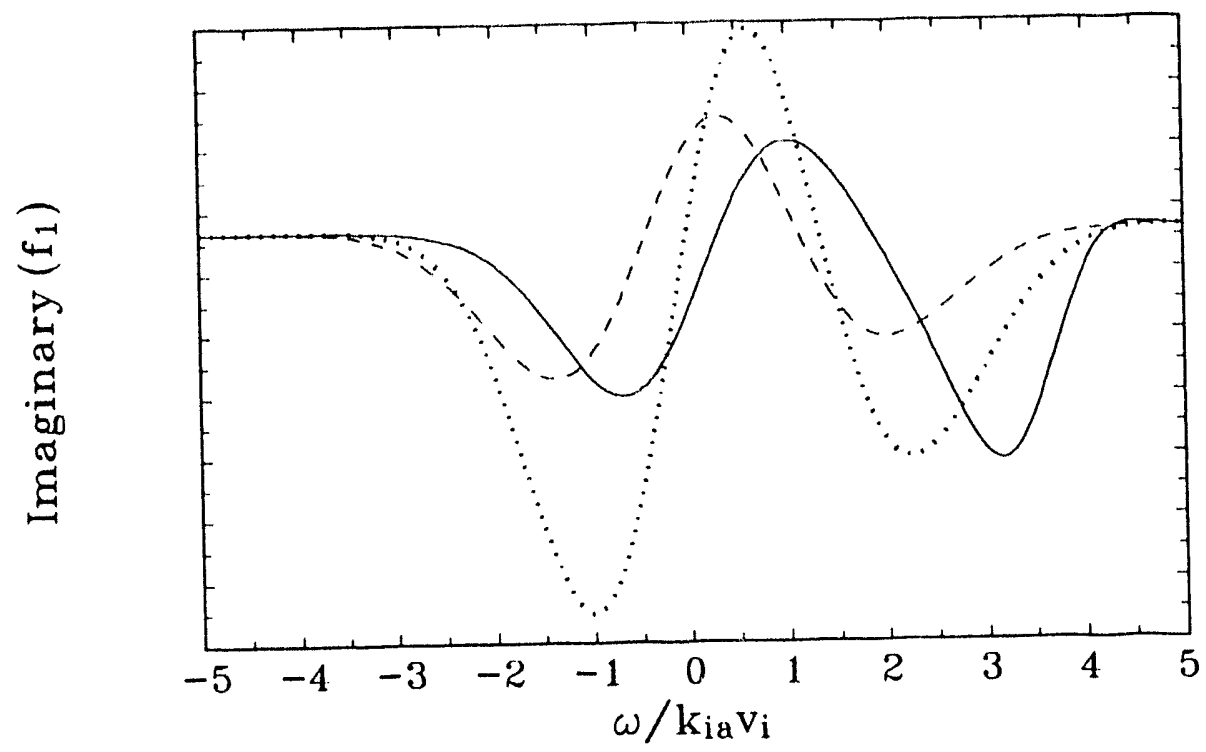

Figure 48: Phase-amplitude normalized imaginary part of the perturbed distribution function for the ion-acoustic wave with $\mathrm{ZT}_{\mathrm{e}} / \mathrm{T}_{\mathrm{i}}=8$ and $\mathrm{k}_{\mathrm{ia}} \lambda_{\mathrm{ii}}=0.133$ (dashes), 0.532 (dots), 2.66 (line).

$\Gamma$ must be found. These quantities are obtained from the perturbed ion distribution function for the ion-acoustic wave [eigenvector with corresponding eigenvalue $\left(\omega_{\mathrm{ia}}{ }^{-}\right.$ $\left.\left.\mathrm{i} \gamma_{\mathrm{ia}}\right) / \mathrm{k}_{\mathrm{ia}} \mathrm{v}_{\mathrm{i}}\right]$ computed by the Fokker-Planck code. The phase-amplitude normalized real and imaginary parts of the perturbed ion distribution function are shown in Figure 47 and Figure 48 , respectively, for the ratio $\mathrm{ZT}_{\mathrm{e}} / \mathrm{T}_{\mathrm{i}}=8$ and various values of $\mathrm{k}_{\mathrm{ia}} \lambda_{\mathrm{ii}}$. The values of $\kappa_{1}, \kappa_{2}, \mu_{1}, \mu_{2}$, and $\Gamma$ are found with the distribution functions, Eq.'s (85) through (89), and the closure relations Eq. (80), and are listed in Table V.

When used in Eq. (119) to find the complex frequencies of the ion-acoustic wave, the values for $\kappa_{1}, k_{2}, \mu_{1}, \mu_{2}$, and $\Gamma$ from Table $V$ must reproduce the real and imaginary 
Table V: Collisionally dependent transport coefficients and ratio of specific heats for the ion-acoustic wave with $\mathrm{ZT}_{\mathrm{e}} / \mathrm{T}_{\mathrm{i}}=8$.

\begin{tabular}{||l|l|l|l|l|l||}
\hline \hline $\mathrm{k}_{\mathrm{ia}} \lambda_{\mathrm{ii}}$ & \multicolumn{1}{|c|}{$\Gamma$} & \multicolumn{1}{|c|}{$\mathrm{K}_{1}$} & \multicolumn{1}{|c|}{$\kappa_{2}$} & \multicolumn{1}{|c|}{$\mu_{1}$} & \multicolumn{1}{c|}{$\mu_{2}$} \\
\hline \hline 0.034 & 1.687 & $-9.57 \mathrm{e}-4$ & 0.1302 & 0.04312 & 0.01182 \\
\hline 0.067 & 1.741 & -.003796 & 0.2387 & 0.08175 & 0.03932 \\
\hline 0.133 & 1.906 & -0.01654 & 0.3843 & 0.1435 & 0.09845 \\
\hline 0.266 & 2.252 & -0.07964 & 0.5121 & 0.2089 & 0.1459 \\
\hline 0.398 & 2.488 & -0.1572 & 0.5490 & 0.2249 & 0.1387 \\
\hline 0.532 & 2.636 & -0.2252 & 0.5532 & 0.2197 & 0.1197 \\
\hline 0.798 & 2.788 & -0.3192 & 0.5351 & 0.1926 & 0.08677 \\
\hline 1.064 & 2.860 & -0.3763 & 0.5145 & 0.1657 & 0.06575 \\
\hline 1.33 & 2.900 & -0.4132 & 0.4989 & 0.1438 & 0.05227 \\
\hline 1.597 & 2.925 & -0.4387 & 0.4861 & 0.1265 & 0.04309 \\
\hline 2.128 & 2.954 & -0.4710 & 0.4692 & 0.1014 & 0.03168 \\
\hline 2.66 & 2.968 & -0.4914 & 0.4577 & 0.08440 & 0.02488 \\
\hline 3.192 & 2.978 & -0.5040 & 0.4498 & 0.07229 & 0.02050 \\
\hline 3.724 & 2.984 & -0.5139 & 0.4443 & 0.06301 & 0.01735 \\
\hline 5.32 & 2.992 & -0.5320 & 0.4342 & 0.04529 & 0.01181 \\
\hline 10.64 & 2.998 & -0.5531 & 0.4212 & 0.02328 & 0.005670 \\
\hline 21.28 & 3.000 & -0.5643 & 0.4148 & 0.01170 & 0.002749 \\
\hline 42.56 & 3.000 & -0.5695 & 0.4113 & 0.005848 & 0.001349 \\
\hline 85.12 & 3.000 & -0.5720 & 0.4096 & 0.002854 & .0006523 \\
\hline & & & & & \\
\hline
\end{tabular}

parts of the ion-acoustic frequency predicted by the Fokker-Planck code in order to be self-consistent. The comparison for the imaginary part (damping) is shown in Figure 49 where the solid line $(-)$ is the damping predicted by the Fokker-Planck code and the circles (O) are the damping results predicted by Eq. (119) with the values for $\kappa_{1}, \kappa_{2}$, 


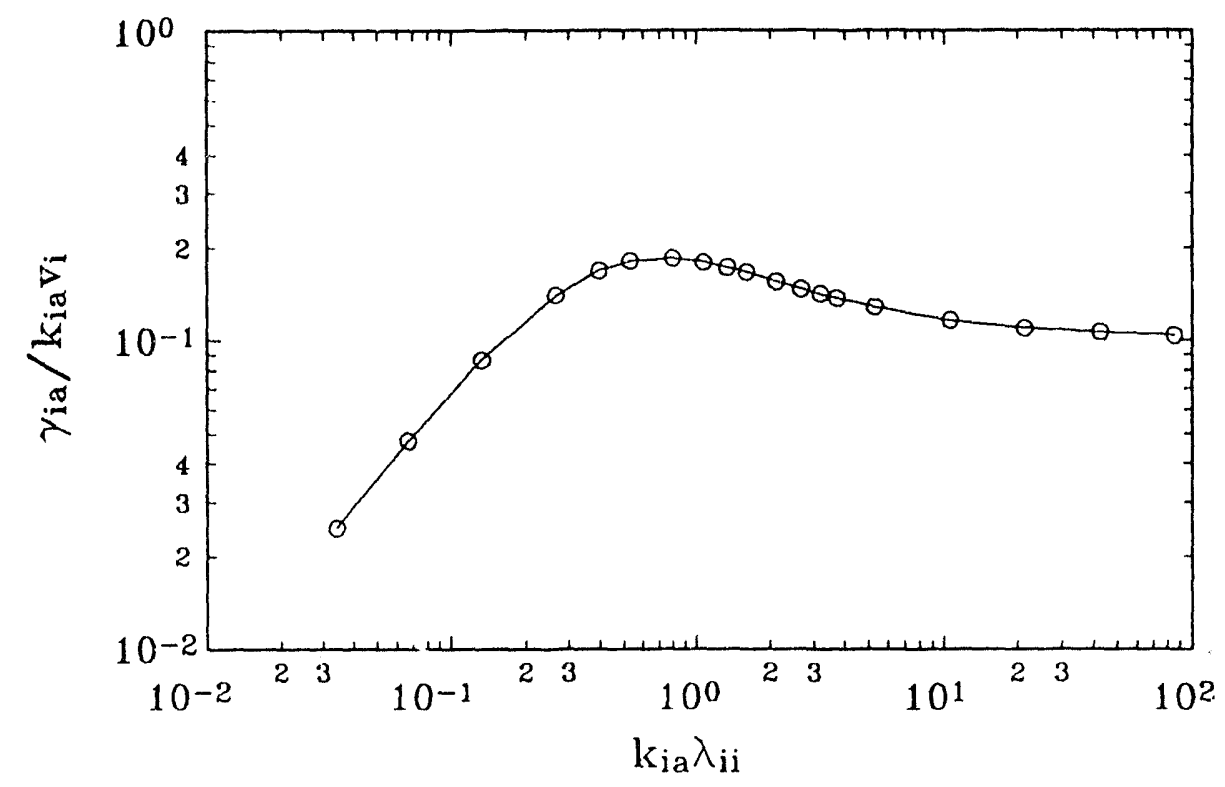

Figure 49: The ion-acoustic damping calculated from the modified fluid model (circles) compared to the exact damping from the eigenvalue solution of the FokkerPlanck equation (line) for $\mathrm{ZT}_{\mathrm{e}} / \mathrm{T}_{\mathrm{i}}=8$.

$\mu_{1}, \mu_{2}$, and $\Gamma$ from Table V. The damping values from the Fokker-Planck code and Eq. (119) agree to the third significant figure, and agreement to the fourth significant figure is obtained for the real part of the ion-acoustic frequency, which is not shown.

Since the complex frequencies of the ion-acoustic wave are accurately reproduced over the entire range of ion-collisionality $\left(\mathrm{k}_{\mathrm{ia}} \lambda_{\mathrm{ii}}\right)$ with the modified fluid equation approach, the peak height asymmetry may be predicted accurately. This is again accomplished with the general equation for the dynamical form factor (Eq. (93)) which gives the spectral distribution in the ion-acoustic Thomson spectrum. The electron susceptibility in the dynamical form factor is expressed by Eq. (92) and the ion 


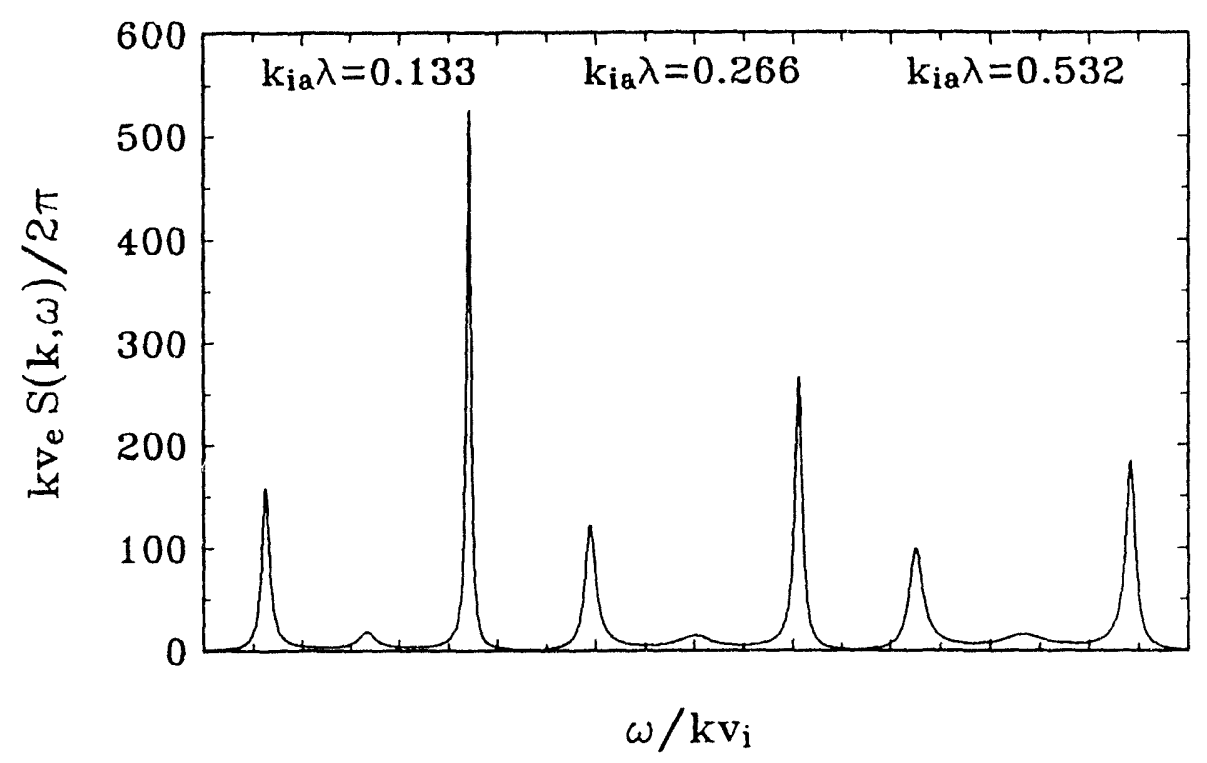

Figure 50: Peak height asymmetry variation with the ion collisionality $\left(\mathrm{k}_{\mathrm{ia}} \lambda_{\mathrm{ij}}\right)$ for fixed $\mathrm{ZT}_{\mathrm{e}} / \mathrm{T}_{\mathrm{i}}=8$ and relative drift $\left(\mathrm{k}_{\mathrm{ia}} \cdot \mathrm{U}_{\mathrm{d}}\right) / \mathrm{k}_{\mathrm{ia}} \mathrm{v}_{\mathrm{e}}=0.02$.

susceptibility is given by Eq. (91). For an ionization/temperature ratio of $\mathrm{ZT}_{\mathrm{e}} / \mathrm{T}_{\mathrm{i}}=8$, the values of $\kappa_{1}, k_{2}, \mu_{1}, \mu_{2}$, and $\Gamma$ in the ion susceptibility are given as a function of collisionality $\left(\mathrm{k}_{\mathrm{ia}} \lambda_{\mathrm{ii}}\right)$ in Table $\mathrm{V}$. The dependence of the peak height asymmetry for a fixed relative drift $\left(U_{d}\right)$ on the collisionality can been seen in Figure 50, where the ionacoustic spectra predicted by the model are shown for $\mathrm{ZT}_{\mathrm{e}} / \mathrm{T}_{\mathrm{i}}=8,\left(\mathrm{k}_{\mathrm{ia}} \cdot \mathrm{U}_{\mathrm{d}}\right) / \mathrm{k}_{\mathrm{ia}} \mathrm{v}_{\mathrm{e}}=$ 0.02 and values of $\mathrm{k}_{\mathrm{ia}} \lambda_{\mathrm{ii}}=0.133,0.266$, and 0.532 . For all three cases, the Landau damping contribution from the electrons is fixed while the collisional damping from the ions gradually increases from 0.0866 at $\mathrm{k}_{\mathrm{ia}} \lambda_{\mathrm{ii}}=0.133$ to 0.181 at $\mathrm{k}_{\mathrm{ia}} \lambda_{\mathrm{ii}}=0.532$ (see Table IV for ion damping). As seen by Figure 50, the peak height asymmetry for the 
given relative drift decreases dramatically as the ion damping contribution increases over the small range of collisionality represented in the figure. This dependence is expected since the peak height asymmetry is due to a reduced/enhanced electron Landau damping of the ion-acoustic waves from the relative drift. The reduction/enhancement in the electron Landau damping occurs for the ion-acoustic wave that is traveling with/against the relative drift respectively, and the amount of electron Landau damping compared to the total damping (electron Landau + ion collisional) determines the sensitivity of the peak height asymmetry to the relative drift. In other words, for a given change in the electron Landau damping due to a relative drift, the total damping of the ion-acoustic wave will be affected more for the cases where the electron Landau damping is a large part of the total damping.

The modified fluid model developed in this chapter can now be applied to the ionacoustic Thomson spectra taken in Chapter 4. Figure 51 shows the Thomson data (dashed line) from a position in the plasma $(\mathrm{z}=200 \mu \mathrm{m}, \mathrm{r}=-25 \mu \mathrm{m})$. The measured quantities at this position from the Thomson spectrum are electron density $\left[\mathrm{n}_{\mathrm{e}}=2.4 \times 10^{19} \mathrm{~cm}^{-3}\right]$ and sound speed $\left[\left(c_{s} / v_{i}\right)=20\right]$. Using the ionization balance model presented in Chapter 4 , the sound speed measurement indicates an electron temperature of $T_{e}=42 \mathrm{eV}$ and an average ionization state of $\mathrm{Z}=7.9$. With $\mathrm{k}_{\mathrm{ia}}=180787 \mathrm{~cm}^{-1}$ for the Thomson measurements (see Eq. (48)), $\alpha=1 / \mathrm{k}_{\mathrm{ia}} \lambda_{\mathrm{DE}}=5.6$ and the ion-ion and electron-ion collisionality given by equation (10) are $\mathrm{k}_{\mathrm{ia}} \lambda_{\mathrm{ii}}=0.705$ and $\mathrm{k}_{\mathrm{ia}} \lambda_{\mathrm{ei}}=4.7$, respectively. The value for $\mathrm{k}_{\mathrm{ia}} \lambda_{\mathrm{ei}}$ allows the plasma at this point to be approximated by collisionless electrons and the value of $\alpha$ allows for the assumption of quasineutrality. Since the 




Figure 51: Modified fluid model fit (solid line) to Thomson spectra (dashed line) from a point in the plasma with axial position $=200 \mu \mathrm{m}$, radial position $=-25 \mu \mathrm{m}$.

conditions of quasineutrality and collisionless electrons approximately hold, the modified fluid model can be used to find the relative drift from the peak height asymmetry. The best fit from the modified fluid model to the Thomson spectrum with $\mathrm{ZT}_{\mathrm{e}} / \mathrm{T}_{\mathrm{i}} \approx 8$ and $\mathrm{k}_{\mathrm{ia}} \lambda_{\mathrm{ii}}=0.705$ is shown in Figure 51 (solid line). The discrepancy in the peak widths between the modified fluid model and the experimental data in Figure 51 is due to broadening of the experimental peaks from instrument response, finite $\Delta \mathrm{k}_{\mathrm{ia}}$ collection, and nonstationarity of the plasma quantities during the duration of the $266 \mathrm{~nm}$ diagnostic pulse. Assuming the relative drift is directed radially, the scattering geometry for the Thomson experiments yields $\left(\mathrm{k}_{\mathrm{ia}} \cdot \mathrm{U}_{\mathrm{d}}\right) / \mathrm{k}_{\mathrm{ia}} \mathrm{v}_{\mathrm{e}}=\left(\mathrm{U}_{\mathrm{d}} / \mathrm{v}_{\mathrm{e}}\right) \cos \left(67.5^{\circ}\right)$ and the best fit is 
accomplished with a normalized value for the relative drift of $\left(U_{d} / v_{e}\right)=0.029$ or $\left(U_{d} / c_{s}\right)$ $=2.1$. The second normalization shows the relative drift is on the order of the sound speed. This value for the relative drift can be contrasted with the relative drift predicted from the return current due to the radial temperature gradient measured in the plasma. Combining equations (78) and (79) in Chapter 4 , the normalized relative drift $\left[U_{d} / v_{e}\right]$ due to the radial temperature gradient $\left(\nabla \mathrm{T}_{\mathrm{e}}\right)$ is given by,

$$
\frac{U_{d}}{v_{e}}=\frac{9 \pi}{640}\left(\frac{\gamma_{T}}{\gamma_{e} \delta_{T} \epsilon}\right) \frac{\kappa^{e} \nabla T_{e}}{n_{e} \nu_{e} T_{e}} \approx \frac{9 \pi}{640}(3.1) \frac{8 \lambda_{e t} \nabla T_{e}}{T_{e}}
$$

where the approximation is made by substituting the values for $\left(\gamma_{\mathrm{T}} / \gamma_{\mathrm{e}} \delta_{\mathrm{T}} \epsilon\right)=3.1$ and $\mathrm{k}^{\mathrm{e}}$ $=8 \mathrm{n}_{\mathrm{e}} \mathrm{v}_{\mathrm{e}} \lambda_{\mathrm{ei}}$ when $\mathrm{ZT}_{\mathrm{e}} / \mathrm{T}_{\mathrm{i}}=8$ (Ref. 147). The normalized relative drift predicted by Eq. (120) with $\nabla \mathrm{T}_{\mathrm{e}}=1400 \mathrm{eV} / \mathrm{cm}$ from the experimental data is $\left(\mathrm{U}_{\mathrm{d}} / \mathrm{v}_{\mathrm{e}}\right)=0.00095$ which is a factor of 30 smaller than the relative drift given by the best fit of the model to the peak height asymmetry of the data. The discrepancy between the two values for the relative drift could be a result of the drift not being directed radially at that point in the plasma and/or mechanisms other than heat transport driving a relative drift such as laser beam nonuniformities in the plasma generating beam. For example if the drift where directed along the ion-acoustic wave, then the $\cos \left(67.5^{\circ}\right)=0.38$ factor used in calculating $\left(\mathrm{U}_{\mathrm{d}} / \mathrm{v}_{\mathrm{e}}\right)$ from the peak height asymmetry would be a factor of 3 smaller. The discrepancies in the Thomson data presented here and at the end of Chapter 4 deserve further investigation. A potential experimental configuration for performing the investigation systematically is discussed in the next chapter.

In this chapter, a modified fluid model has been developed that can be used to 
measure the relative drift velocity between the thermal electrons and ions from the peak height asymmetry in a Thomson spectra when given the values for the ionization/temperature ratio $\mathrm{ZT}_{\mathrm{e}} / \mathrm{T}_{\mathrm{i}}$ and ion-collisionality $\mathrm{k}_{\mathrm{ia}} \lambda_{\mathrm{ii}}$. The transport coefficients and the ratio of specific heats that appear in the model are found as a function of $\mathrm{ZT}_{\mathrm{e}} / \mathrm{T}_{\mathrm{i}}$ and $\mathrm{k}_{\mathrm{ia}} \lambda_{\mathrm{ii}}$ using the perturbed ion distribution functions from the eigenvalue solution of the linearized Fokker-Planck equation. Since the modified fluid model correctly reproduces the complex frequencies of the ion-acoustic wave for the entire range of ioncollisionality $\left(\mathrm{k}_{\mathrm{ia}} \lambda_{\mathrm{ii}}\right)$ in the quasineutral limit $(\alpha \rightarrow \infty)$ with collisionless electrons, the model is self-consistent. The limitations for the current model are the need for collisionless electrons $\left(\mathrm{k}_{\mathrm{ia}} \lambda_{\mathrm{ei}} * 1\right)$, quasineutrality $(\alpha * 1)$, and weak ion coupling $\left(\ln \Lambda_{\mathrm{ii}}\right.$ *1). When the quantities $\mathrm{k}_{\mathrm{ia}} \lambda_{\text {ei }}$ and/or $\alpha$ are approximately $\leq 1$, the effects of electron collisions and/or non-quasineutrality must be included through the Fokker-Planck equation for calculating the transport coefficients and the ratio of specific heats. Also, the FokkerPlanck equation does not account for the large angle scattering effects when the ions become strongly coupled. The strongly coupled condition occurs when the ion-ion Coulomb logarithm is of order $1\left(\ln \Lambda_{\mathrm{ii}} \approx 1\right)$. These conditions add complexity to the solution of the Fokker-Planck equation which is beyond the scope of this dissertation. Finally, the modified fluid model does not at this point accurately predict the ion-acoustic spectrum near zero frequency where the entropy wave feature resides. With the information obtained from the Fokker-Planck results regarding the entropy wave, it should be possible to develop a model to reliably calculate the spectrum of the entropy wave in the future. 
Chapter VI: Experimental configuration II (peak asymmetry investigation)

In this chapter, changes will be proposed in the experimental configuration described in Chapter 3 which will allow for a systematic investigation of peak height asymmetries in collective Thomson spectra. As mentioned at the end of Chapter 4, the peak height asymmetries in the laser-produced plasma studied in this dissertation do not correlate as one might expect with the radial temperature gradients measured in the plasma. Although the peak asymmetry in the data collected for Chapter 4 was reproducible in some instances, it was not reproducible in general. A reproducible asymmetry would suggest the possibility of relative drifts driven by beam nonuniformities in the plasma generating beam. Since the peak asymmetry is not always reproducible, the signal averaging techniques used for measurement of the electron density, sound speed, and radial drift in Chapter 4 will not allow for acquisition of meaningful peak height asymmetry data. Therefore, the collective Thomson scattering data must be collected on a single shot basis for a proper analysis. At the same time, since there is a question concerning the relationship between peak height asymmetry and the radial temperature gradients in the plasma, Thomson scattering data from multiple radial points should be collected simultaneously. The Thomson scattering collection system shown in Figure 17 of Chapter 3 only allows for single point measurements in the plasma and is obviously incompatible with the requirements imposed by single shot measurement of the entire radial temperature gradient. The conditions of single shot, multiple point data acquisition can be satisfied by modifying the Thomson scattering collection optics, astigmatic 
compensation of the spectrometer in the collection system, and replacing the onedimensional reticon detector on the back of the spectrometer with a two-dimensional CCD detector.

The detector used for acquiring the collective ion-acoustic Thomson spectrum for electron density, sound speed, and radial drift velocity measurements is an 1024-element single-intersified reticon array (EG\&G Model 1420UV). As discussed in the experimental configuration of Chapter 3 , this detector is mounted at the exit plane of a 0.85 meter double monochrometer (SPEX Model 1404) which spectrally disperses the Thomson scattered light onto the detector. The reticon detector has an overall quantum efficiency (Q.E.) $\approx 10 \%$ at the Thomson scattering wavelength of $266 \mathrm{~nm}$. This means that for every 10 phorons incident per pixel element on the detector, 1 count is registered. For a typical Thomson scattering shot, 70 counts are registered per pixel at peak maximum. This implies that for an average single Thomson scattering plasma shot, assuming a Q.E. $\approx 10 \%, 700$ photons are incident on each $2.5 \mathrm{~mm}$ high by $25 \mu \mathrm{m}$ wide element of the detector. The signal-to-noise $(\mathrm{S} / \mathrm{N})$ of the Thomson peaks for a single shot is only $\approx 3: 1$. The relatively low signal-to-noise is the reason for integrating 10 shots per point measurement in the plasma. By averaging 10 shots, the signal-to-noise is improved by approximately a factor of $\sqrt{10}$. The single shot signal-to-noise obtained with the reticon detector would introduce an error of $30 \%$ in the peak height asymmetry measurements. For this reason, the Princeton Instruments Intensified CCD detector (Model ICCD) is chosen to replace the reticon detector for the peak height asymmetry measurements. The specifications for the efficiency of this two-dimensional detector are, 


$$
\begin{gathered}
1 \text { photoelectron }\left(p e^{-}\right)=80 \text { counts } \\
\text { Q.E. of }\left(p e^{-}\right) \text {generation from photons }=15 \%(\text { at } 266 \mathrm{~nm})
\end{gathered}
$$

Since the CCD detector is made up of a two-dimensional grid of $25 \mu \mathrm{m} \times 25 \mu \mathrm{m}$ detector elements, $100 \mathrm{CCD}$ pixels is equivalent to one reticon element. Using the previously estimated 700 photons incident on each reticon pixel per plasma shot, an equivalent Thomson experiment using the CCD detector would generate a signal,

$$
\text { Signal }=700 \text { photons } / 100 \text { pixels } / \text { shot }
$$

Since the Q.E. Kor photoelectron generation from Eq. (121) is $15 \%$,

$$
\text { Signal }=105 \text { pe-/100 pixels/shot }
$$

Also from Eq. (121), 80 counts are generated for every photoelectron, therefore,

$$
\text { Signal }=8400 \text { counts } / 100 \text { pixels } / \text { shot }
$$

The total noise $\left(\mathrm{N}_{\mathrm{T}}\right)$ associated with the signal of Eq. (124) is given by,

$$
N_{T}=\sqrt{N_{R}^{2}+N_{E B I}^{2}+N_{D}^{2}+N_{p h}^{2}}
$$

where $N_{R}$ is the read out noise, $N_{E B I}$ is the photocathode noise, $N_{D}$ is the dark noise of the CCD, and $\mathrm{N}_{\mathrm{ph}}$ is the photon shot noise. For the CCD detector, the read out noise is $N_{R}=1$ count and for the intensifier in the gated mode, $N_{E B I} \approx 0$. The dark noise of the CCD is given by, $N_{D}=1$ count/pixel/(second of readout time), and the photon shot noise is equal to the square root of the number of photoelectrons generated, $\mathrm{N}_{\mathrm{ph}}=(\#$ pe)$^{1 / 2}$. From Eq. (121), 105 photoelectrons are generated for each equivalent reticon element of the CCD detector during a Thomson spectrum acquisition, therefore the 
photon shot noise associated with the signal is, $N_{p h}=(105)^{1 / 2}=10.2$ pe-. Since 80 counts are registered for every photoelectron, $N_{\mathrm{ph}}=820$ counts/shot. Using $100 \mathrm{CCD}$ pixels in one shot with an average read out time of $1 \mathrm{sec}$, the total noise associated with the Thomson signal on the CCD detector is given by,

$$
N_{T}=\sqrt{1^{2}+0^{2}+100^{2}+820^{2}}=826 \text { counts } R M S
$$

The signal-to-noise obtained in the Thomson spectrum by the CCD detector under equivalent conditions to that of the reticon detector is now found through the ratio of Eq. (124) to Eq. (126),

$$
S / N=\frac{\text { Signal }}{N_{r}}=\frac{8400 \text { counts }}{826 \text { counts } R M S} \approx 10
$$

Therefore, the CCD will collect a single shot Thomson spectrum with a signal-to-noise comparable to that obtained with the reticon detector by averaging 10 plasma shots. A signal-to-noise of 10 will introduce an acceptable $10 \%$ error in the peak height asymmetry measurements.

In addition to providing reasonable signal-to-noise levels in a single shot Thomson spectrum, the CCD detector also solves the second problem associated with the peak height asymmetry analysis, multiple point data acquisition. Since the detector is twodimensional, one axis of the detector can be used to record the spectral content of the Thomson scattered light from a given point in the plasma, while the other axis can be used to monitor the spatial information from multiple radial positions in the plasma with an appropriately modified collection and imaging system.

The collection system from Chapter 3 must be modified slightly to allow for the 




Figure 52: Optical schematic of the collection system which couples the Thomson scattered signal from the plasma onto the CCD detector for the peak height asymmetry analysis.

acquisition of Thomson scattered light simultaneously from several radial positions. Figure 52 shows the proposed collection system for the peak height asymmetry analysis. The Thomson scattered light is collected with an $300 \mathrm{~mm}$ focal length (f/12) lens and imaged with a magnification of 5 onto the entrance slit of the spectrometer. The image is rotated in between the lens and entrance slit by making a right angle turn through a two mirror periscope. The rotation is necessary to align the image of the horizontal dia $b_{b}$ nstic beam, and therefore the scattered light, parallel with the vertical entrance slit of the spectrometer. The scattered light is also sent through a $266 \mathrm{~nm}$ zero-order, half-wave $(\lambda / 2)$ plate before reaching the entrance slit. The half-wave plate is used to rotate the 
polarization of the scattered light to a vertical orientation which minimizes polarization losses in the spectrometer. With this arrangement, different points along the entrance slit correspond to different radial positions in the plasma along the scattered diagnostic beam. The spectrometer disperses the light passing through the entrance slit onto the CCD detector. Each point from the slit, which corresponds to a radial scattering point from the plasma, produces a Thomson spectrum along the wavelength axis on the CCD detector, and the Thomson spectrum from different radial points are stacked along the position axis on the CCD detector as shown in Figure 52.

A magnification of $x 5$ in the collection system is chosen to optimize the spatial resolution of the CCD detector along the radial direction in the plasma, while at the same time not affecting the wavelength resolution of the system. The $20 \mu \mathrm{m}$ diameter diagnostic beam is imaged to a $100 \mu \mathrm{m}$ diameter beam at the $100 \mu \mathrm{m}$ wide entrance slit. The beam image diameter matches the entrance slit width so there is optimum coupling of the scattered light into the spectrometer. With a magnification of 5 , each $25 \mu \mathrm{m}$ high element in the $\mathrm{CCD}$ detector corresponds to $5 \mu \mathrm{m}$ in the radial direction in the plasma. The CCD detector has about a 4 pixel cross-talk which means that a point image will appear to be 4 pixels in diameter on the detector. This cross-talk limits the spectral resolution in the wavelength direction from $0.032 \AA /$ pixel to about 0.12 angstroms and limits the radial resolution from $5 \mu \mathrm{m} /$ pixel to about $20 \mu \mathrm{m}$. Because $100 \mu \mathrm{m}$ along spectral axis corresponds exactly with the 4 pixel cross-talk, the $100 \mu \mathrm{m}$ entrance slit is optimum for use with the CCD detector. A larger entrance slit width would only produce additional spectral broadening in the Thomson scattered peaks, and lower the resolution. Because 
the entrance slit width is fixed, an increase in the magnification of the collection system would improve the radial position resolution of the CCD detector, but at the expense of light collection efficiency due to imperfect coupling of the diagnostic beam image with the entrance slit width. Since there is cross-talk, the amount of light integrated for each radial Thomson spectra can be improved by grouping three rows of elements on the CCD array as one radial position. As seen in Figure 52, the groups of three rows are separated by two rows of elements and in this fashion several radial Thomson spectra can be recorded at the same time with a center separation of $25 \mu \mathrm{m}$ in the plasma.

The extent to which the CCD detector can be used to make two-dimensional measurements of scattered wavelength vs. radial position in the plasma relies sensitively on the imaging properties of the spectrometer. Most spectrometers only provide low aberration image compensation from the entrance slit to the exit plane along the dispersion or wavelength axis because aberrations along the axis orthogonal to the dispersive direction do not affect spectral measurements. The spectrometer used in the previous experimental configuration of Chapter 3 exhibits a significant amount of aberration along the orthogonal axis. The source of the aberration can be identified from the optical schematic for the spectrometer shown in Figure 53. The spectrometer is a flatfield imager but due to off-axis reflections from the interior curved mirrors, the system is astigmatic. The astigmatism causes the dispersion and orthogonal axes to have different image planes. For reference, the dispersion axis is in the plane of the diagram and the orthogonal axis points out of the paper. The astigmatism can be compensated for by introducing a long focal length (10 meter f.1.) cylindrical lens into the optical path 




Figure 53: Optical schematic of the spectrometer used for the Thomson scattering experiments. A cylindrical lens is added to provide astigmatic compensation for twodimensional imagin

between the final tur

or and image plane. When properly orientated, the lens will only affect the position of the image plane for the orthogonal axis. The image plane for the orthogonal axis can then be made to coincide with the position of the image plane for the dispersion axis. The method for aligning the compensating lens is as follows; First, the entrance slit is illuminated by the $253 \mathrm{~nm}$ line from a mercury $(\mathrm{Hg})$ calibration lamp and the $\mathrm{CCD}$ detector is aligned with the image plane of the spectrometer. The CCD detector coincides with the image plane when the measured spectral width of the $253 \mathrm{~nm}$ line is a minimum. Next, the entrance slit is replaced with a wire mesh with $100 \mu \mathrm{m}$ diameter wires on a $150 \mu \mathrm{m}$ center-to-center spacing. The mesh is illuminated with the 
$253 \mathrm{~nm} \mathrm{Hg}$ line and orientated so the wires are parallel to both the dispersion and orthogonal axis. The $253 \mathrm{~nm} \mathrm{Hg}$ line is used for alignment of the CCD detector and compensating lens because of its proximity to the wavelength of the Thomson scattered light at $266 \mathrm{~nm}$. Since the focal length of the compensating lens is wavelength dependent, illumination with the $253 \mathrm{~nm}$ line will allow for proper placement of the lens for use with the Thomson scattered light. A narrow line width source, like the $253 \mathrm{~nm} \mathrm{Hg}$ line, must also be used to eliminate spectral blurring of the two-dimensional mesh image at the CCD detector. The distance of the mesh from the spectrometer is now changed until the vertical wires from the mesh are brought into focus on the CCD detector. At this point, the mesh is in the original position of the entrance slit. Figure 54 shows the twodimensional image of the mesh recorded by the CCD detector at this point. The vertical wires which are perpendicular to the dispersion axis are in focus while the horizontal wires are badly out of focus due to the astigmatism of the spectrometer. The compensating lens is now inserted and rotated to a position which causes the smallest change in the focus of the vertical lines. The proper rotation position insures that the curvature axis of the cylindrical lens is aligned with the orthogonal astigmatic axis and therefore will only affect the position of the orthogonal axis image plane. The position of the orthogonal axis image plane is made to coincide with position of the dispersion axis image plane by translating the compensating lens along the optical path until the horizontal wires from the mesh come into focus. Since the compensating lens does add some optical path length, the CCD detector position needs to be adjusted slightly to produce the best two-dimensional image of the mesh. Figure 55 shows the improved 


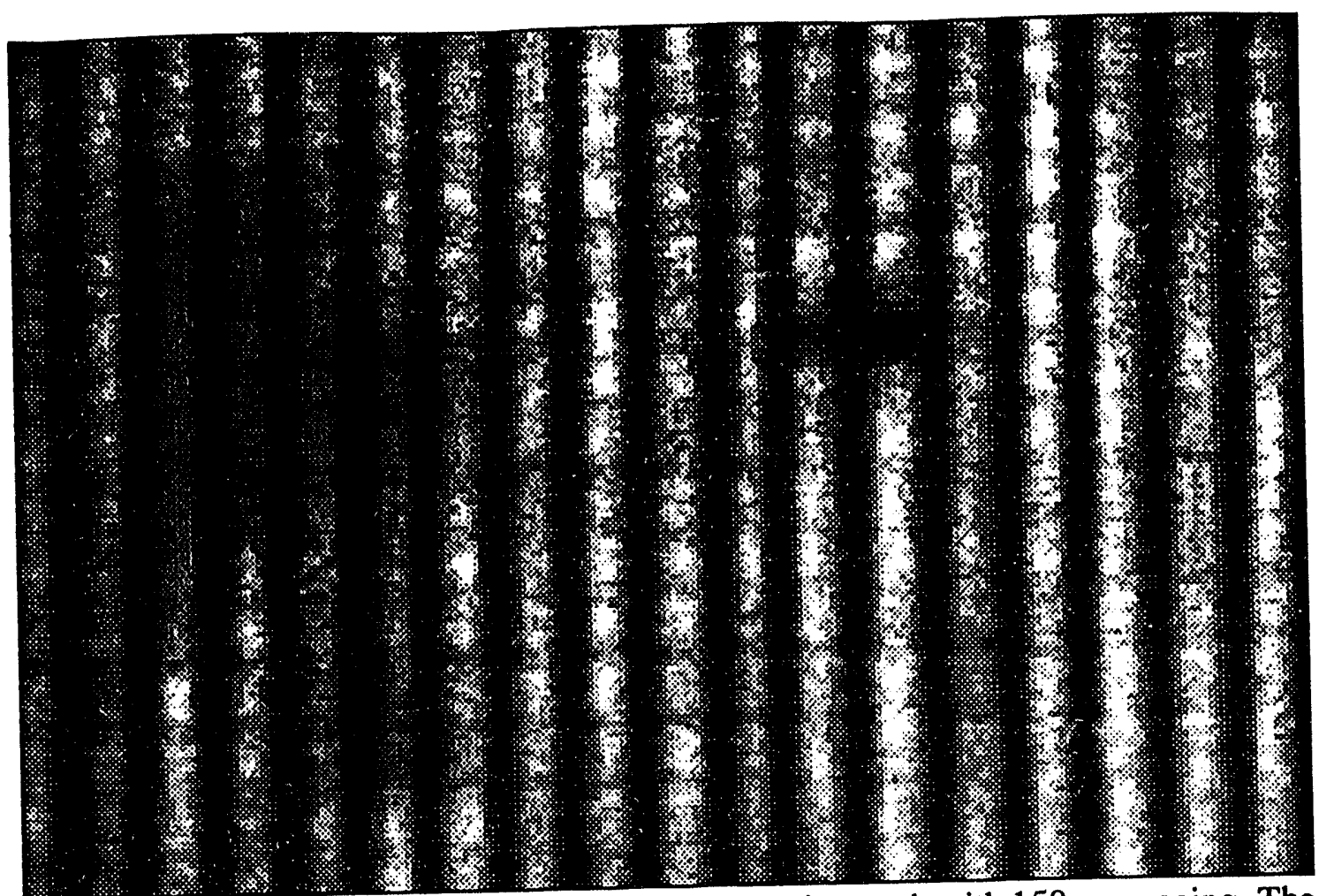

Figure 54: $\mathrm{CCD} /$ Spectrometer imaging of $100 \mu \mathrm{m}$ wire mesh with $150 \mu \mathrm{m}$ spacing. The horizontal wires are not visible due to the astigmatism of the spectrometer.

image of the wire mesh from the astigmatic compensated spectrometer. Since the $100 \mu \mathrm{m}$ wires are well resolved, the same resolution can be expected for the Thomson scattering experiments when the entrance slit is in place. With the magnification of 5 in the collection system, the effective resolution of the CCD detector in the radial direction in the plasma will be better than $20 \mu \mathrm{m}$.

As a final note, the wire mesh is rotated 45 degrees in Figure 55 for a reason. Since the mesh is a periodic structure, the compensating lens will produce many image planes due to diffraction from the mesh which are separated along the optic axis by a Talbot cycle ${ }^{155}$. Only one of these image planes corresponds with the true geometrical image which contains all of the spatial Fourier components of the mesh pattern. The other 


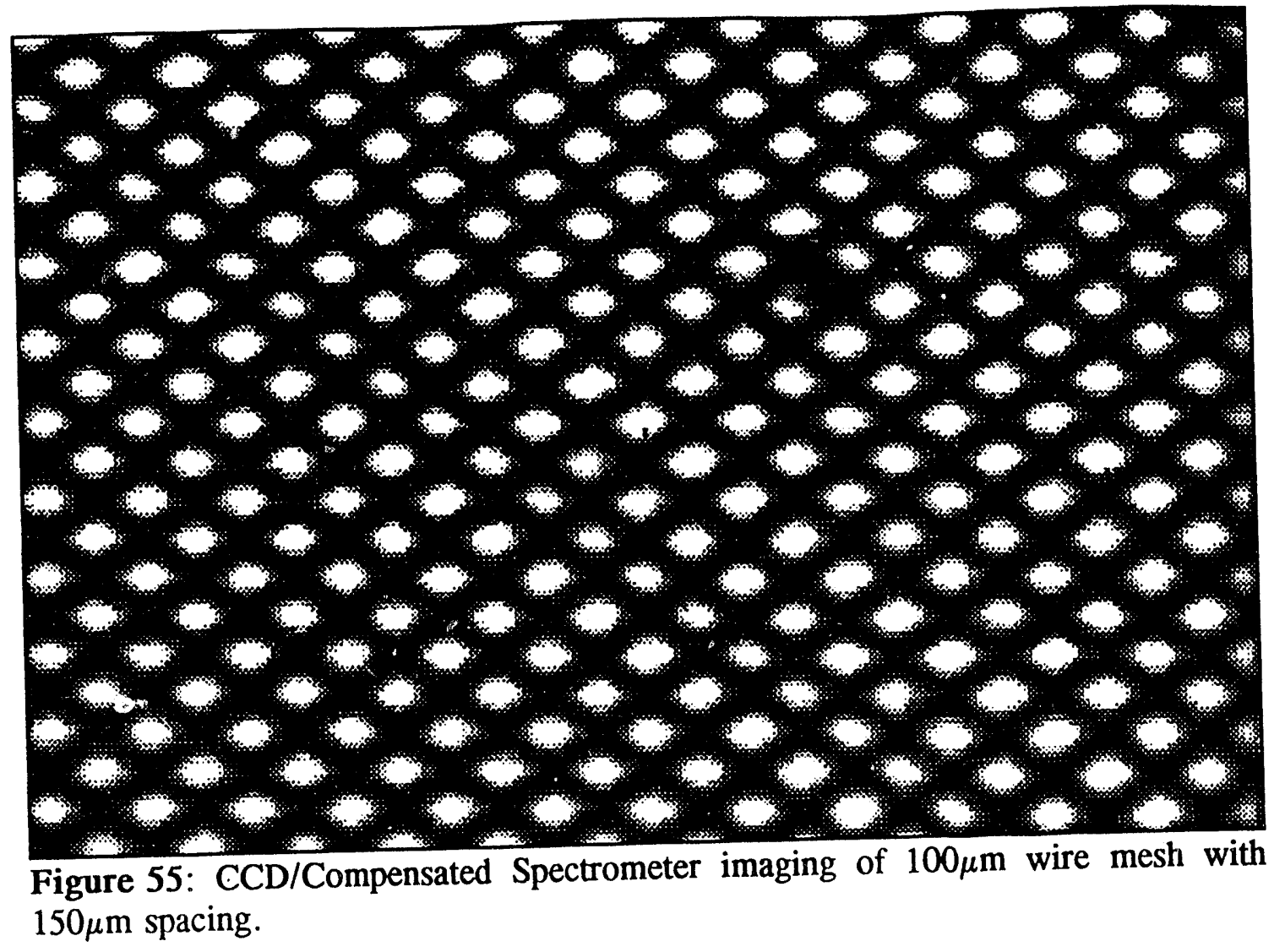

image planes result from reconstructions of only a subset of the Fourier components. The true geometrical image is found when a rotation of the mesh, which produces a change in the spatial Fourier components, does not change the focus quality of the mesh wires on the $\mathrm{CCD}$ detector. One way to avoid the Talbot cycle in aligning the system would be to use a non-periodic structure like a cross-hair.

Other than the collection system changes mentioned here, the experimental configuration and data acquisition procedures which were used to collect the electron density, sound speed, and radial drift data in Chapter 4 will remain the same for the peak height asymmetry experiments. Continued monitoring of the electron density and sound speed is required for an estimation of the ion-ion mean free path $\left(\lambda_{\mathrm{ii}}\right)$. The ion-ion mean 
free path is a function of the electron density, ion temperature, and ionization state of the plasma, see Eq. (10). The electron temperature and ionization state can be obtained from the sound speed data by using the ionization balance model discussed in Chapter 4 , and since the laser plasma is collisional, see Chapter 4 , the ion temperature is approximately equal to the electron temperature. With the ion-ion mean free path, the collisionality $\left(\mathrm{k}_{\mathrm{ia}} \lambda_{\mathrm{ii}}\right)$ of the ion-acoustic wave is known. The theory presented in Chapter 5 can then be used to analyze the peak height asymmetries since the theory is a function of the collisionality $\left(\mathrm{k}_{\mathrm{ia}} \lambda_{\mathrm{ii}}\right)$.

This chapter has presented the changes necessary in the collection system from that described in Chapter 3 for an investigation of the peak height asymmetries in collective Thomson spectra for the laser-produced plasma studied in this dissertation. The new collection system allows for single shot, multiple radial point data acquisition. The data must be collected on a single shot basis because there is some questions as to the shot-toshot reproducibility of the relative thermal drift between the electrons and ions. Multiple radial point data acquisition is necessary for single shot measurements of the radial electron temperature gradient $\left(\nabla \Gamma_{\mathrm{e}}\right)$. With the theory in Chapter 5 , the peak height asymmetry can be related to the relative thermal drift $\left(U_{d}\right)$ between the electrons and ions. The most probable source for the relative drift is a return current driven by a heat flux $\left(q_{e}\right)$. Verification of the direct relaiionship between relative drift $\left(U_{d}\right)$ and the heat flux $\left(q_{e}\right)$ through a consistency check $\left(q_{e}=-k^{e} \nabla T_{e}\right)$ is a very important measurement to make in a laser plasma. If there is not a self-consistent relationship between the relative drift and the heat flux, then other mechanisms may be driving the relative drift, such as 
beam nonuniformities in the plasma generating beam. Nonuniformities or 'hot spots' in the plasma generating beam could be triggering the onset of thermal filamentation in the plasma. The filaments are stochastic and tend to flicker on a time scale much shorter than the $266 \mathrm{~nm}$ diagnostic pulse width of $5 \mathrm{~ns}$. Therefore, the steep temperature gradients produced in the filaments would not be directly detectable under the current Thomson scattering conditions. But, the steep gradients may impulsively drive the relative drifts that are producing the inconsistent peak height asymmetries in the Thomson spectra. This hypothesis could be experimentally investigated by placing a random phase plate (RPP) in the $1.06 \mu \mathrm{m}$ plasma generating beam. The introduction of the RPP would remove beam nonuniformities, and should affect the peak height asymmetries if the hypothesis is correct. 
Chapter VII: Conclusions

Although there exists no universal laser-plasma diagnostic, collective Thomson scattering from ion-acoustic waves has proved to be the most convenient and useful diagnostic for investigating the characteristics of the collisional, laser-produced, aluminum plasma studied in this dissertation. Using the existing Thomson scattering theory reviewed in Chapter 2, it was possible to accurately measure the two-dimensional electron density, sound speed, and radial drift velocity profiles of the laser plasma. The measurements were facilitated by generating a reproducible plasma in the three quantities being measured. The reproducibility of the electron density, sound speed, and radial drift velocity was verified in Chapter 4, and owing to this reproducibility, single point Thomson measurements from different plasma shots could be correlated. This correlation permitted the single point Thomson measurements to be assembled into a two-dimensional picture of the plasma. The reproducibility also allowed for signal averaging to be used which improved the signal-to-noise of the Thomson spectrum from the low density portions of the plasma.

Besides performing the most detailed measurements of a laser-produced plasma to date, the Thomson scattering experiments of this dissertation ${ }^{156,157}$ are the first done at a wavelength as short as $266 \mathrm{~nm}$. The ultraviolet diagnostic wavelength is necessary to minimize the complicating effects of inverse bremsstrahlung and refractive turning in the high density regions of the plasma that are being studied $\left(n_{e} \approx 1 \times 10^{20}\right.$ $\mathrm{cm}^{-3}$ ). As was discussed in Chapter 4, a diagnostic wavelength of $532 \mathrm{~nm}$ would have 
been unacceptable for performing Thomson scattering from the plasma studied in this dissertation. A $532 \mathrm{~nm}$ diagnostic beam would cause local heating of the plasma due to inverse bremsstrahlung which would change the value for temperature dependent sound speed being measured. Also, the refractive turning of a $532 \mathrm{~nm}$ diagnostic beam would limit accessibility in the plasma and reduce the amount of scattered light collected.

The two-dimensional data for the electron density, sound speed, and radial drift velocity was used to benchmark the hydrodynamics code (LASNEX) in the parameter regime represented by the plasma in this dissertation. The laser-plasma studied is produced with a moderate intensity laser pulse $\approx 2 \times 10^{11} \mathrm{~W} / \mathrm{cm}^{2}$ so that instabilities such as SBS and SRS that occur at higher irradiance levels do not develop. Instabilities could complicate the data analysis and comparison with LASNEX and are therefore undesirable for fundamental studies of this type. The laser-plasma is collisional and as shown in Chapter 4 , has an electron-ion mean free path $\left(\lambda_{\mathrm{ei}}\right)$ which is much shorter than the temperature scale lengths $\left(\mathrm{T}_{\mathrm{e}} / \nabla \mathrm{T}_{\mathrm{e}}\right)$ measured from the Thomson scattering data. The condition $\left(\lambda_{e i} * T_{e} / \nabla T_{e}\right)$ is necessary for the plasma to be in the Spitzer regime where classical electron heat transport theory applies. Since L.ASNEX is based on the hydrodynamic fluid equations with classical transport coefficients, the computer model should accurately predict the features of the plasma, and in fact it does. The values for the electron density, sound speed, and radial drift velocity predicterl by LASNEX and measured through collective Thomson scattering all agree within a percent standard deviation of $50 \%$. This type of detailed comparison between a computer model and an experiment are important for raising the confidence level in the accuracy of the computer 
model for predicting the characteristics for other similar plasmas.

A detailed investigation of a plasma allows one to gain a basic understanding of the physical principles governing the plasma and its evolution. In Chapter 4 , simple models based on first principles where presented that demonstrate the self-consistency of the experimentally measured electron density, ound speed, and radial drift velocity. For example, the predicted radial sonic point in the plasma obtained from the steady state equations of motion for the electrons and ions matches the experimentally measured radial sonic point within the error bars of the experiment. The simple models presented not only verify the accuracy of the experimental measurements self-consistently, but also show that some aspects of a very complicated collisional laser-produced plasma can be predicted without a intricate 'black box' code like LASNEX. When possible, a simplistic approach to modeling a plasma is desirable because it permits intuition to be used in understanding the characteristics of the plasma.

Despite the self-consistency of the electron density, sound speed, and radial drift measurements, the peak height asymmetries in the measured ion-acoustic Thomson spectrum do not appear to correlate with the temperature gradients of the plasma. The peak height asymmetry in an ion-acoustic Thomson spectrum is directly related to the relative drift velocity $\left(U_{d}\right)$ between the thermal electrons and ions. As discussed in chapters 4,5 , and 6 , the relative drift is usually thought to be a result of the return current driven by heat transport due to a temperature gradient in the plasma. The first step in a more thorough investigation of the relationship between the peak height asymmetry and the measured temperature gradients involves extending the existing 
Thomson theory. As discussed in Chapters 2 and 5, the existing Thomson scattering theory accurately predicts the peak height asymmetry in the ion-acoustic Thomson spectrum in the collisionless $\left(\mathrm{k}_{\mathrm{ia}} \lambda_{\mathrm{ii}} \rightarrow \infty\right)$ and collisional $\left(\mathrm{k}_{\mathrm{ia}} \lambda_{\mathrm{ii}} \rightarrow 0\right)$ plasma regimes for a given relative drift $\left(U_{d}\right)$ and ionization/temperature ratio $\left(\mathrm{ZT}_{\mathrm{e}} / \mathrm{T}_{\mathrm{i}}\right)$. For plasmas of intermediate collisionality $\left(\mathrm{k}_{\mathrm{ia}} \lambda_{\mathrm{ii}} \approx 1\right)$, like the one studied in this dissertation, a new theory had to be developed to accurately predict the peak height asymmetry for a given collisionality $\left(\mathrm{k}_{\mathrm{ia}} \lambda_{\mathrm{ii}}\right)$, relative drift $\left(\mathrm{U}_{\mathrm{d}}\right)$, and ionization/temperature ratio $\left(\mathrm{ZT}_{\mathrm{e}} / \mathrm{T}_{\mathrm{i}}\right)$. The new theory is presented in Chapter 5 and is based on the fluid equations with modified closure relations. The collisionally dependent transport coefficients $\left(\kappa_{1}, \kappa_{2}, \mu_{1}, \mu_{2}\right)$ and ratio of the specific heats $(\Gamma)$ that appear in the closure relations and the fluid equations are found from the perturbed ion distribution function for the ion-acoustic wave. The perturbed ion distribution function is in turn calculated from the eigenvalue solution of the Fokker-Planck equation ${ }^{158}$. The new theory which is termed 'the modified fluid model', self-consistently reproduces the real and imaginary parts of the complex frequency for the ion-acoustic wave for any value of $\mathrm{k}_{\mathrm{ia}} \lambda_{\mathrm{ii}}$ and $\mathrm{ZT} \mathrm{T}_{\mathrm{e}} / \mathrm{T}_{\mathrm{i}}$, and will therefore accurately predict the change in the damping of the ion-acoustic waves due to a relative drift which produces the peak height asymmetry in the Thomson spectrum. The current limitations of the modified fluid model are the electrons must be collisionless $\left(\mathrm{k}_{\mathrm{ia}} \lambda_{\mathrm{ei}} * 1\right)$, quasineutrality must hold $(\alpha * 1)$, and the ions must be weakly coupled $\left(\ln \Lambda_{\mathrm{ii}} * 1\right)$.

Armed with the modified fluid model, an experimental configuration is presented in Chapter 6 that can be used to investigate the relationship between the relative drift $\left(U_{d}\right)$, measured from the peak height asymmetry, and the temperature gradient, obtained 
from the spatially dependent sound speed. The experiments will be performed on a single shot basis with the Thomson scattered signal collected simultaneously from several different radial positions in the plasma. The single shot detection is facilitated with an intensified CCD camera and is necessary to eliminate any reproducibility issues related to the plasma. The CCD camera is required for the simultaneous multiple point detection of the Thomson signal which will be used to measure the spatially dependent sound speed.

The results from the proposed peak height asymmetry experiments are expected to address the issue of heat transport in a collisional, laser-produced plasma. If the relative drift measurements do not correlate with the expected values from classical transport theory, then some mechanism other than the return current driven by heat transport may be causing the relative drift, for example nonuniformities in the plasma generating beain. One important reason for investigating the mechanisms which generate a relative drift in the plasma is that the relative drift can drive ion-acoustic waves above thermal levels by what is referred to as the relative drift instability. The ion-acoustic waves could then act as a seeding mechanism for SBS. Regardless of the outcome, the peak height asymmetry experiments will certainly result in the first detailed spatially dependent relative drift measurements of a laser-produced plasma. This information can be added to the electron density, sound speed, and radial drift measurements conducted for this dissertation to yield even a greater understanding of the physics associated with a laser-produced plasma. 
Appendix: Code for eigenvalue solution of the Fokker-Planck equation

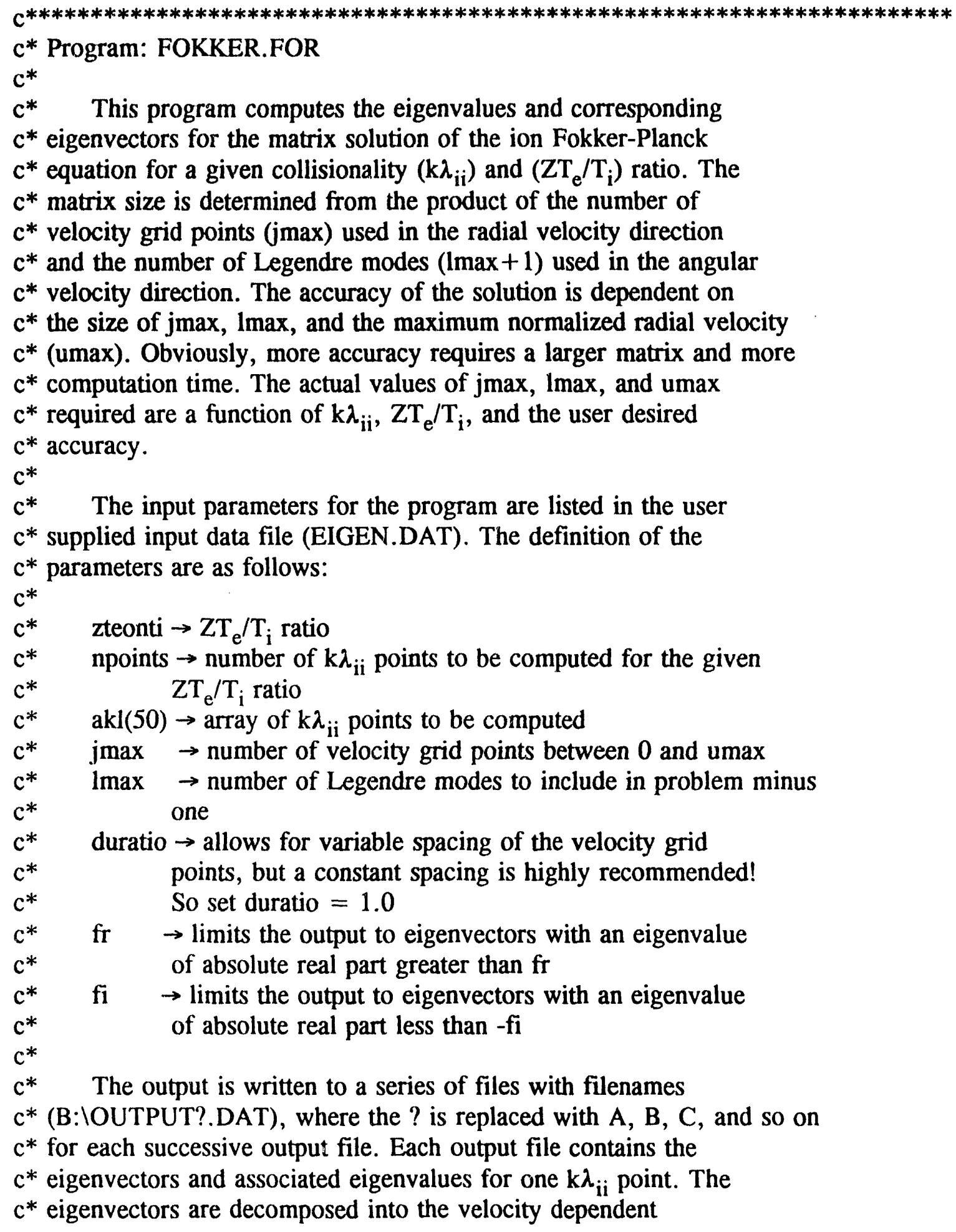


$c^{*}$ coefficients of the Legendre modes from 0 to umax listed in order $\mathrm{c}^{*}$ from Legendre mode $=0$ to mode $=1 \mathrm{max}$. The eigenvector is only $c^{*}$ written to the file if its eigenvalue is the lowest damped zero $c^{*}$ frequency solution (entropy wave) or if the absolute value of the $c^{*}$ real part of the eigenvalue falls between fr and -fi (ion-acoustic $c^{*}$ wave).

$c^{*}$

$c^{*} \quad$ Other important variables for the code are:

$c^{*}$

c* $\quad \operatorname{ar}(600,600) \rightarrow$ real part of the matrix to be solved

$c^{*} \quad$ ai $(600,600) \rightarrow$ imaginary part of the matrix to be solved

$c^{*} \quad \operatorname{wr}(600) \rightarrow$ real part of the eigenvalues

$c^{*} \quad$ wi $(600) \rightarrow$ imaginary part of the eigenvalues

c* $\quad \operatorname{zr}(600,600) \rightarrow$ column matrix of real part of eigenvectors

$c^{*} \quad \mathrm{zi}(600,600) \rightarrow$ column matrix of imaginary part of eigenvectors

$\mathrm{c}^{*} \quad$ ui(200) $\rightarrow$ radial velocity grid

$c^{*}$

c*

c*

c*

$c^{*}$

c*

c*

c*

$c^{*}$

$c^{*}$

c*

c*

$c^{*}$

c*

c*

$c^{*}$

c*

$c^{*}$

c*

c*

c* One additional note - All variables are in double precision!

CG(nm,n,ar,ai,wr, wi, matz,zr,zi,fv1,fv2,fv3,ierr)

CBABK2(nm, n, low, igh,scale, $m, \mathbf{z r}, \mathrm{zi}$ )

CBAL(nm, n,ar, ai, low,igh,scale)

$\operatorname{CDIV(ar,ai,br,bi,cr,ci)}$

COM2(nm,n,low, igh, ortr,orti, hr, hi,wr, wi,zr,zi,ierr)

CORTH(nm,n,low, igh, ar, ai, ortr,orti)

CSROOT(xr, xi,yr,yi)

PYTHAG(a,b)

The subroutines written for the error function calculation:

ERFUN(x)

GAMMP(a,x)

GAMMLN(xx)

GSER(gamser,a,x,glm)

GCF(gammcf,a,x,gln)

$\mathrm{c}^{* *}$

REAL*8 ar $(600,600), a i(600,600), \operatorname{wr}(600), \operatorname{wi}(600), f v 1(600)$

REAL*8 fv2 $(600)$, fv3 $(600)$,term,term 1, akion, el,t1, el1,el2

REAL*8 zr $(600,600), z \mathrm{z}(600,600)$

REAL*8 ui(200), fiO(200),rl(200),r2(200), fr,fi,du,erfun

REAL*8 usum, umax, zteonti, uarg,duratio, rtp, trp, tr2, fact 
REAL*8 akion 1, ak,bk,damp,akl(50), dux INTEGER r

eye $=$ CMPLX $(0,1$.

तtp $=$ SQRT(2./3.1415926535)

trp $=3 .{ }^{*}$ SQRT (3.1415926535)

tr $2=3 .{ }^{*}$ SQRT (2.)

c. read input parameters

$\operatorname{OPEN}(10$, file = 'eigen.dat' $)$

$\operatorname{READ}(10, *)$ zteonti

$\operatorname{READ}\left(10,{ }^{*}\right)$ npoints

do $600 \mathrm{i}=1$, npoints

$\operatorname{READ}(10, *)$ akl(i)

600 continue

$\operatorname{READ}\left(10,{ }^{*}\right)$ jmax

$\operatorname{READ}\left(10,{ }^{*}\right) \operatorname{Imax}$

$\operatorname{READ}\left(10,{ }^{*}\right)$ umax

$\operatorname{READ}(10, *)$ duratio

$\operatorname{READ}(10, *) \mathrm{fr}$

$\operatorname{READ}(10, *)$ fi

CLOSE(10)

c.. set up velocity grid

$$
\begin{aligned}
& d u=1 . \\
& \text { usum }=.5 \\
& \text { do } 3 \mathrm{j}=2 \text {, jmax } \\
& \mathrm{du}=\mathrm{du} * \text { duratio } \\
& \text { usum }=\text { usum }+\mathrm{du}
\end{aligned}
$$

c.. set up maxwellian rosenbluth potentials

c. . $\mathrm{rl}=\mathrm{dH} / \mathrm{dv} ; \mathrm{r} 2=\mathrm{dG} / \mathrm{dv}$ 
do $71 \mathrm{j}=1$,jmax

$r l(j)=2 .^{*}\left(r^{*}\right.$ ui $\left.(j) * f i 0(j)-e r f u n(u i(j) / S Q R T(2)).\right) / u i(j) * * 2$

$r 2(j)=\operatorname{erfun}(u i(j) / S Q R T(2))+.r l(j) / 2$.

71 continue

c. . begin main loop

DO $700 \mathrm{ii}=1$, npoints

akion $=\operatorname{akl}($ ii $)$

c. intialize matrix

do $91 \mathrm{j}=1, \mathrm{jmax} *(\max +1)$

do $92 \mathrm{k}=1, \mathrm{jmax} *(\operatorname{lmax}+1)$

$$
\begin{aligned}
& \operatorname{ar}(\mathbf{j}, \mathbf{k})=0.0 \\
& \operatorname{ai}(\mathbf{j}, \mathbf{k})=0.0
\end{aligned}
$$

92 continue

91 continue

akion $1=1.0$

c. . set up matrix

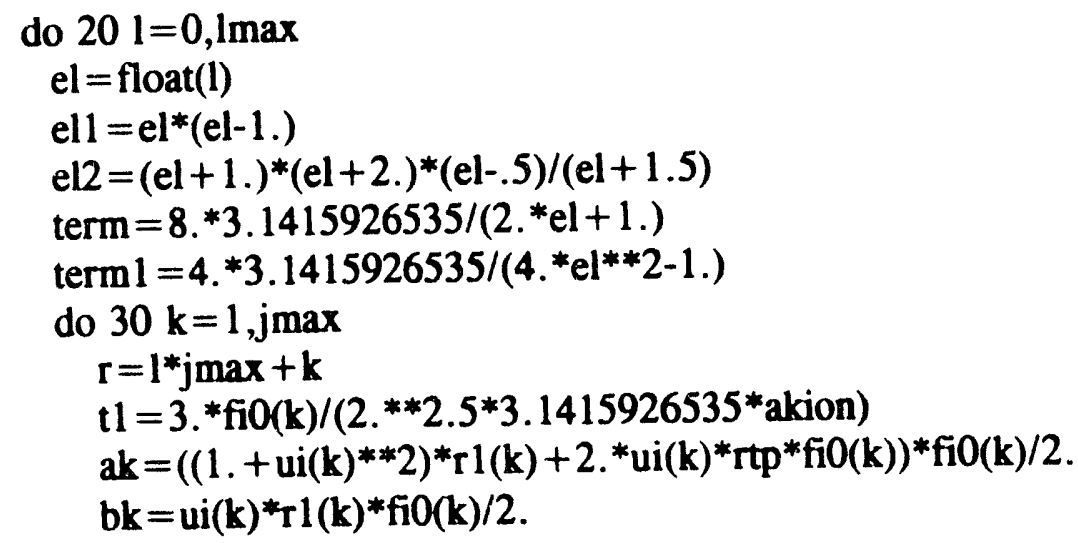

c.. diagonal term

$$
\begin{aligned}
& \text { ai(r,r) }=\text { ai }(r, r)+t r 2 * \text { fio }(k) / \text { akion-trp*r } 2(k) * e^{*}(e l+1 .) / \\
& \&\left(2 .^{*}\right. \text { akion*ui(k)*3) }
\end{aligned}
$$

c.. side diagonal terms

IF (r-jmax.gt.0) $\operatorname{ar}(r, r-j \max )=\operatorname{ar}(r, r-j \max )+\left(e l^{*} u i(k) /(\right.$ 
\&2.*(el-1.)+1.))*akion 1

IF $(r+j \max . l e . j \max *(\max +1)) \operatorname{ar}(r, r+j \max )=\operatorname{ar}(r, r+j \max )+$ $\&\left((\mathrm{el}+1 .)^{*} \mathrm{ui}(\mathrm{k}) /\left(2 .{ }^{*}(\mathrm{el}+1 .)+1 .\right)\right)^{*}$ akion 1

c.. derivative tern

IF (k.ne.1) THEN

IF(k.ne.2) THEN

IF(k.ne.jmax) THEN

IF(k.ne.jmax-1) THEN ai $(r, r-2)=a i(r, r-2)+t_{p}^{*}(a k / 12 .+b k /(12 . * d u x)) /(f i 0($

\&k-2)*akion*ui(k)**2*dux) $\mathrm{ai}(\mathrm{r}, \mathrm{r}-1)=\mathrm{ai}(\mathrm{r}, \mathrm{r}-1)-\operatorname{trp} *\left(2 .{ }^{*} \mathrm{ak} / 3 .+4 .{ }^{*} \mathrm{bk} /\left(3 .{ }^{*} \mathrm{dux}\right)\right) /$ $\&($ fio(k-1)*akion*ui(k)**2*dux)

ai $(r, r)=a i(r, r)+$ trp $^{*} 5 .{ }^{*}$ bk $/\left(2{ }^{*}\right.$ fio $\left.(k) * a k i o n * u i(k) * * 2 * d u x * * 2\right)$

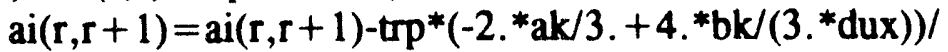

$\&\left(\right.$ fio $(k+1) *$ akion*ui $(k)^{* *} 2 *$ dux $)$ ai $(r, r+2)=a i(r, r+2)-\operatorname{trp}^{*}\left(\mathrm{ak} / 12 .-\mathrm{bk} /\left(12 .{ }^{*} \mathrm{dux}\right)\right) /(\mathrm{fiO}($

$\& \mathrm{k}+2) *$ akion*ui( $(\mathrm{k}) * * 2 *$ dux $)$

ELSE

$\operatorname{ai}(r, r+1)=\operatorname{ai}(r, r+1)-\operatorname{trp} *\left(-a k / 4 .+11 .{ }^{*} b k /\left(12 .{ }^{*} d u x\right)\right) /(f i 0($

$\& k+1) *$ akion*ui $(k) * * 2 * d u x)$ $\mathrm{ai}(\mathrm{r}, \mathrm{r})=\mathrm{ai}(\mathrm{r}, \mathrm{r})+\mathrm{trp} *\left(5{ }^{*} \mathrm{ak} / 6 .+5 .{ }^{*} \mathrm{bk} /\left(3 .{ }^{*} \mathrm{dux}\right)\right) /(\mathrm{fiO}(\mathrm{k}) *$

\&akion*ui(k)**2*dux) $\mathrm{ai}(\mathrm{r}, \mathrm{r}-1)=\mathrm{ai}(\mathrm{r}, \mathrm{r}-1)-\mathrm{trp} *\left(3 .{ }^{*} \mathrm{ak} / 2 .+\mathrm{bk} /\left(2 .^{*} \mathrm{dux}\right)\right) /(\mathrm{fi} 0(\mathrm{k}-1)$

$\& *$ akion*ui(k)**2*dux) $\mathrm{ai}(\mathrm{r}, \mathrm{r}-2)=\mathrm{ai}(\mathrm{r}, \mathrm{r}-2)-\mathrm{trp} *\left(-\mathrm{ak} / 2 .+\mathrm{bk} /\left(3 .{ }^{*} \mathrm{dux}\right)\right) /\left(\mathrm{fiO}(\mathrm{k}-2)^{*}\right.$

\&akion*ui( $(\mathrm{k}) * * 2 *$ dux $)$ ai $(r, r-3)=a i(r, r-3)-t^{*} *\left(a k / 12 .-b k /\left(12 .{ }^{*} d u x\right)\right) /\left(f i 0(k-3)^{*}\right.$

\&akion*ui(k)**2*dux)

ENDIF

ELSE

ai $(r, r)=a i(r, r)-t r p^{*}\left(-11 .{ }^{*} a k / 6 .+2 .{ }^{*} b k / d u x\right) /(f i 0(k) * a k i o n *$ \&ui $(\mathbf{k})^{* * 2 *}$ dux)

ai $(r, r-1)=a i(r, r-1)-\operatorname{trp}^{*}\left(3 .{ }^{*}\right.$ ak $-5 .{ }^{*}$ bk $/$ dux $) /\left(\right.$ fiO $(k-1)^{*}$

\&akion*ui(k)**2*dux)

ai $(r, r-2)=\operatorname{ai}(r, r-2)-\operatorname{trp}^{*}\left(-3{ }^{*} \mathrm{ak} / 2 .+4 .^{* b k} / \mathrm{dux}\right) /\left(\mathrm{fiO}(\mathrm{k}-2)^{*}\right.$

\&akion*ui(k)**2*dux)

ai $(r, r-3)=a i(r, r-3)-t r p *(a k / 3 .-b k / d u x) /(f i 0(k-3) * a k i o n *$

\&ui $(\mathbf{k})^{* * 2 *}$ dux)

ENDIF

ELSE

ai $(r, r-1)=a i(r, r-1)-t r p *\left(a k / 4 .+11 .^{* b k} /\left(12 .{ }^{*}\right.\right.$ dux $\left.)\right) /(f i O($

\&k-1)*akion*ui(k)**2*dux) 


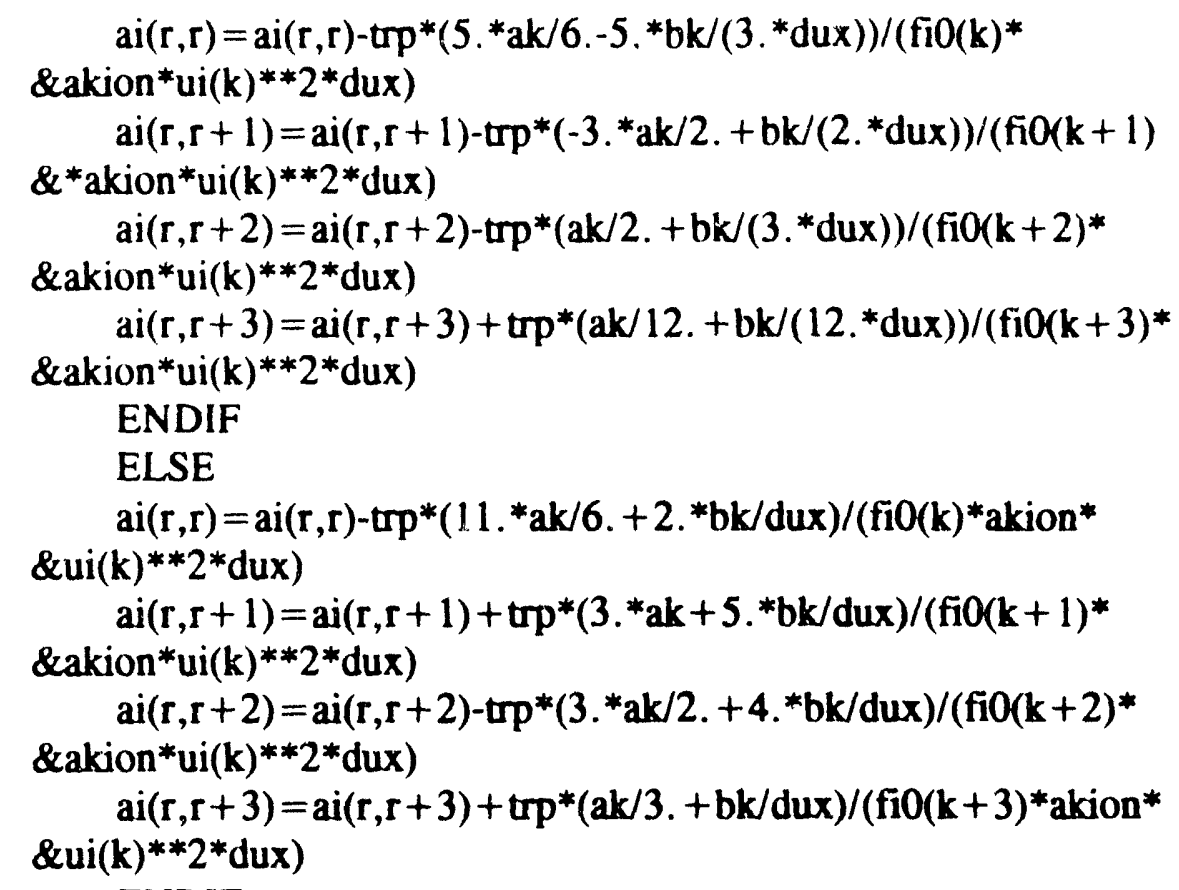

ENDIF

c.. fOonf1 term (vprime $<v)$

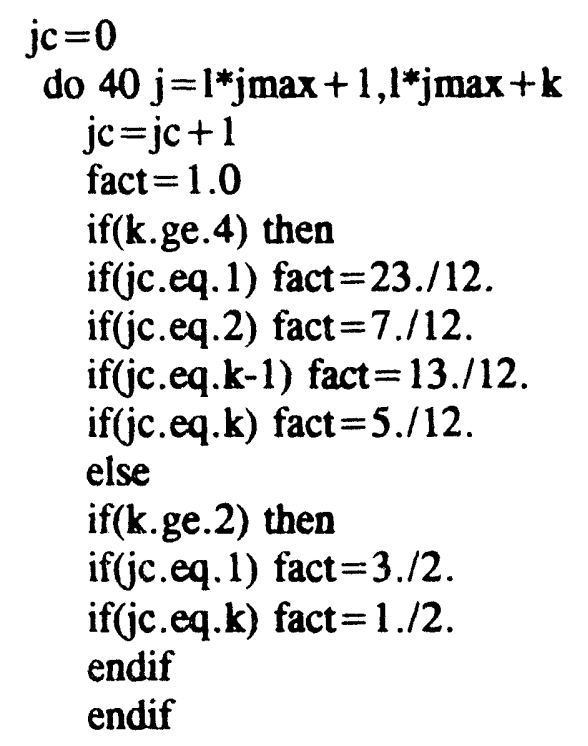

c.. h potential

$\&{ }^{*}$ dux $*_{\text {fact }}$

$$
\operatorname{ai}(r, j)=a i(r, j)-t{ }^{*} \text { term*ui }(j c) *(u i(j c) / u i(k)) * *(l+1)
$$

c.. g potential 


$$
\begin{aligned}
& \text { ai }(r, j)=a i(r, j)-t l * \text { term } 1 * u i(j c) * * 3 *(e l l *(u i(j c) / \\
& \text { \&ui } \left.(\mathbf{k}))^{* *}(1-1)-\mathrm{el} 2^{*}(\mathrm{ui}(\mathrm{jc}) / \mathrm{ui}(\mathbf{k}))^{* *}(1+1)\right)^{*} \mathrm{dux} * \mathrm{fact}
\end{aligned}
$$

40 continue

c.. foonfl term (vprime $>$ v)

$$
\begin{aligned}
& \mathrm{jc}=\mathrm{k}-1 \\
& \text { if(k.eq.jmax) goto } 900 \\
& \text { do } 42 \mathrm{j}=\mathrm{l}^{*} \mathrm{j} \max +\mathrm{k},(1+1)^{*} \mathrm{j} \max \\
& \mathrm{jc}=\mathrm{jc}+1 \\
& \text { fact }=1.0 \\
& \text { if(k.le.jmax-3) then } \\
& \text { if }(\text { jc.eq.k).or. (jc.eq.jmax })) \text { fact }=5 . / 12 \text {. } \\
& \text { if }((j c . e q . k+1) \cdot \text { or } .(j c . e q . j \max -1)) \text { fact }=13 . / 12 \text {. } \\
& \text { else } \\
& \text { if(k.le.jmax-1) then } \\
& \text { if ((jc.eq.k).or.(jc.eq.jmax })) \text { fact }=1 . / 2 \text {. } \\
& \text { endif } \\
& \text { endif }
\end{aligned}
$$

c.. h potential

$$
\underset{\text { ai }(r, j)=a i(r, j)-t 1{ }^{*} \operatorname{term} * u i(j c) *(u i(k) / u i(j c)) * * 1}{\text { fact }}
$$

c.. g potential

$$
\begin{aligned}
& \operatorname{ai}(r, j)=\operatorname{ai}(r, j)-t 1 * \operatorname{term} 1 * u i(j c) * * 3 *(e l 1 *(u i(k) / \\
& \text { \&ui } \left.(j \mathrm{c}))^{* *} \mathrm{l}-\mathrm{el} 2 *(\mathrm{ui}(\mathrm{k}) / \mathrm{ui}(\mathrm{jc}))^{* *}(1+2)\right)^{*} \mathrm{dux} * \text { fact }
\end{aligned}
$$

42 continue

900 continue

c.. ion density term








20 continue

WRITE $\left({ }^{*},{ }^{*}\right)$ 'made it!'

c.. find eigenvalues and eigenvectors

$\mathrm{n}=\mathrm{jmax} *(\operatorname{lmax}+1)$

matz $=0$

call $\operatorname{cg}(\mathrm{n}, \mathrm{n}, \mathrm{ar}, \mathrm{ai}, \mathrm{wr}, \mathrm{wi}, \mathrm{matz}, \mathrm{zr}, \mathrm{zi}, \mathrm{fv} 1, \mathrm{fv} 2, \mathrm{fv} 3$, ierr)

c.. write solutions to output files

c.. write entropy wave eigenvector

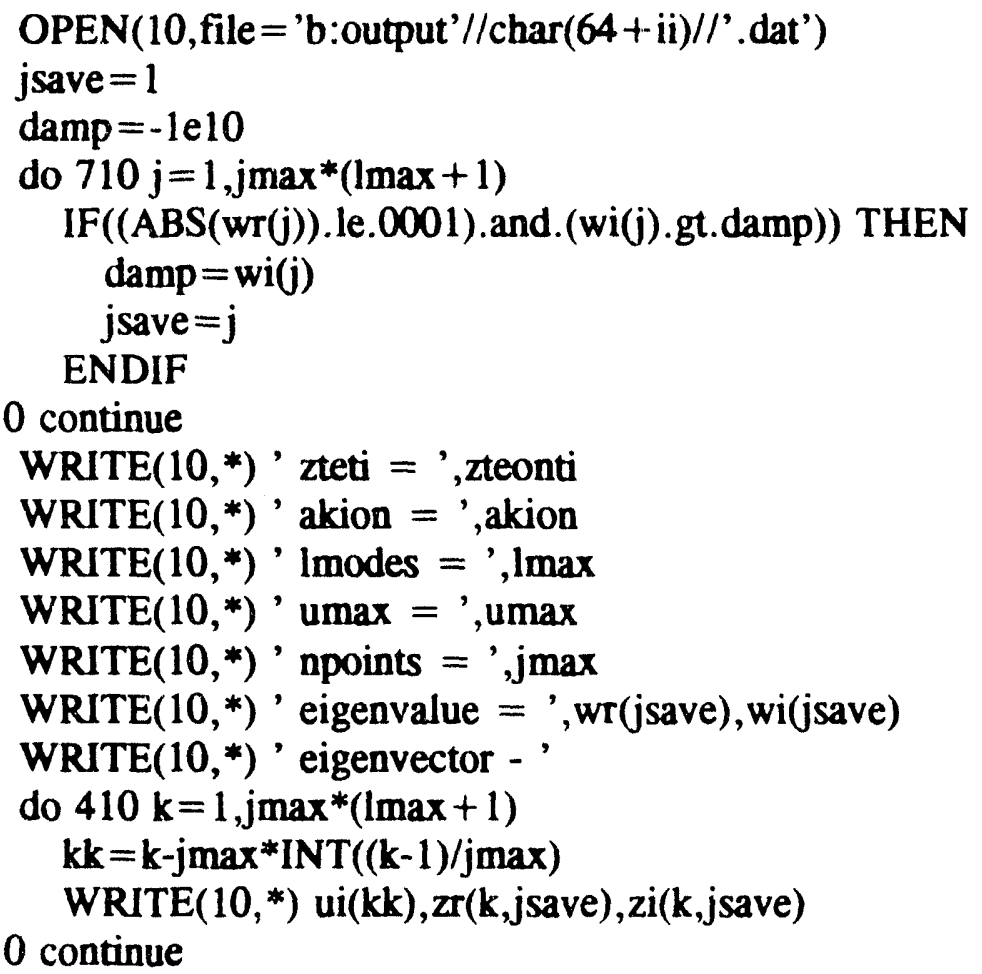

c.. write ion-acoustic eigenvectors 


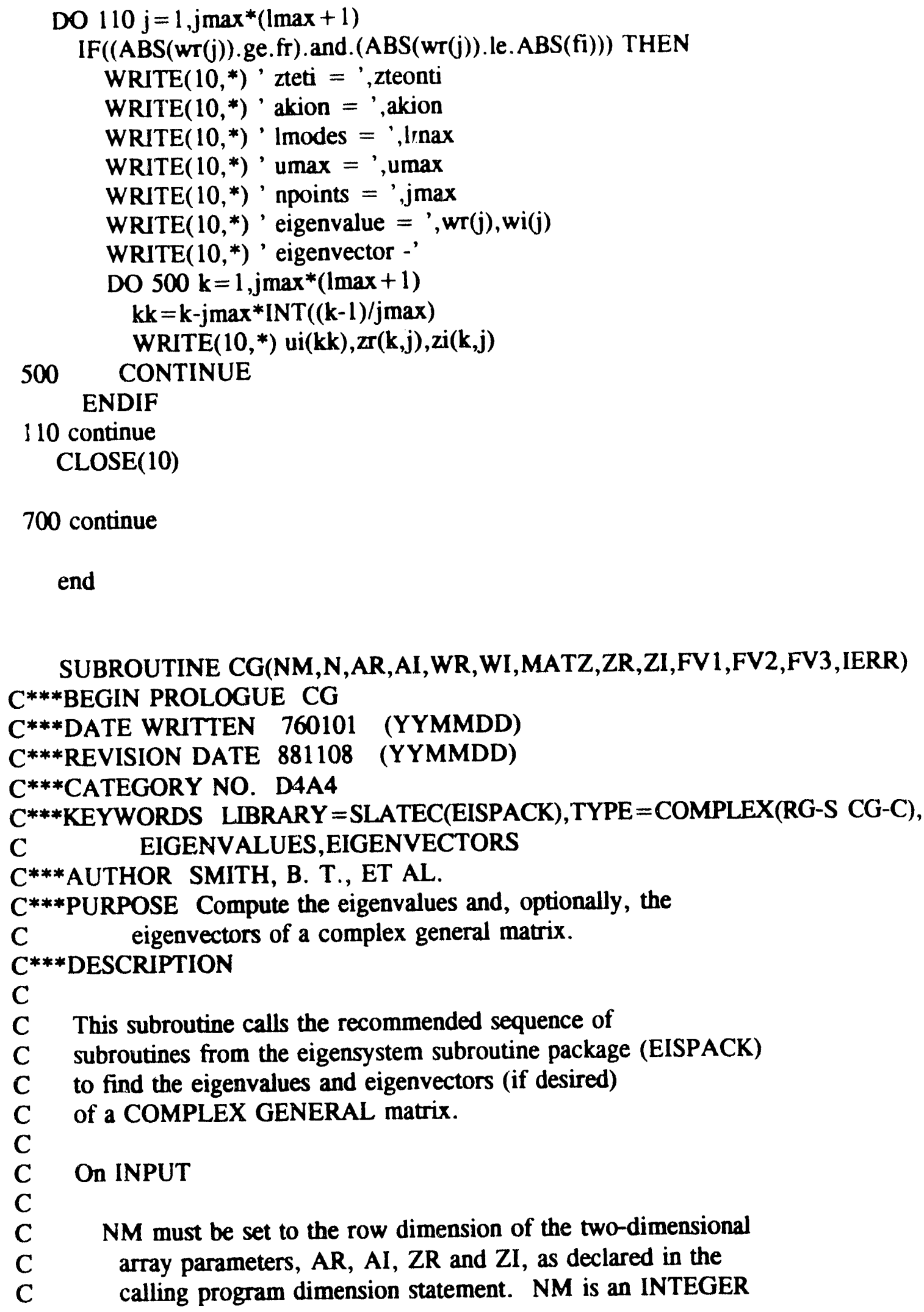









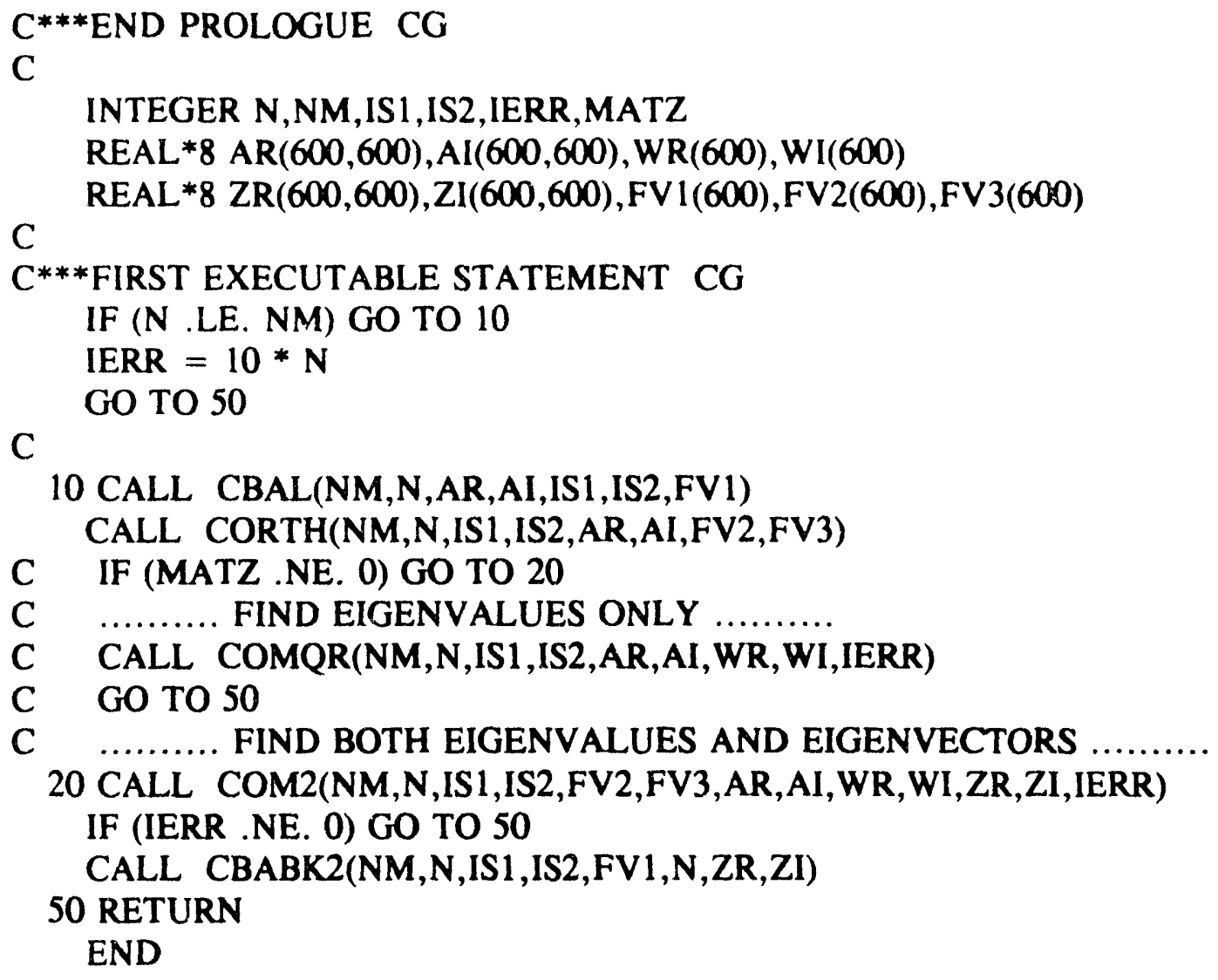

SUBROUTINE CBABK2(NM,N,LOW,IGH,SCALE,M,ZR,ZI)

$C^{* * * B E G I N ~ P R O L O G U E ~ C B A B K 2}$

$C^{* * *}$ DATE WRITTEN 760101 (YYMMDD)

C***REVISION DATE 881108 (YYMMDD)

C***CATEGORY NO. D4C4

C**KEYWORDS LIBRARY $=$ SLATEC $($ EISPACK $), T Y P E=C O M P L E X(B A L B A K-S$

CBABK2-C),

C EIGENVALUES,EIGENVECTORS

C***AUTHOR SMITH, B. T., ET AL.

$C^{* * *}$ PURPOSE Form the eigenvectors of a complex general matrix from

$\mathrm{C}$ the eigenvectors of matrix output from CBAL.

C***DESCRIPTION

$\mathrm{C}$

C This subroutine is a translation of the ALGOL procedure

C CBABK2, which is a complex version of BALBAK,

C NUM. MATH. 13, 293-304(1969) by Parlett and Reinsch.

C HANDBOOK FOR AUTO. COMP., VOL.II-LINEAR ALGEBRA, 315-326(1971).

C

C This subroutine forms the eigenvectors of a COMPLEX GENERAL

C matrix by back transforming those of the corresponding 
C balanced matrix determined by CBAL.

C

C On INPUT

C

C NM must be set to the row dimension of the two-dimensional

$\mathrm{C}$ array parameters, ZR and ZI, as declared in the calling

C program dimension statement. NM is an INTEGER variable.

C

C

$\mathrm{N}$ is the order of the matrix $\mathrm{Z}=(\mathrm{ZR}, \mathrm{ZI}) . \quad \mathrm{N}$ is an INTEGER

variable. $\mathbf{N}$ must be less than or equal to $\mathrm{NM}$.

LOW and IGH are INTEGER variables determined by CBAL.

SCALE contains information determining the permutations and scaling factors used by CBAL. SCALE is a one-dimensional REAL array, dimensioned SCALE(N).

$M$ is the number of eigenvectors to be back transformed. $M$ is an INTEGER variable.

ZR and ZI contain the real and imaginary parts, respectively, of the eigenvectors to be back transformed in their first

$M$ columns. ZR and ZI are two-dimensional REAL arrays, dimensioned $\mathrm{ZR}(\mathrm{NM}, \mathrm{M})$ and $\mathrm{ZI}(\mathrm{NM}, \mathrm{M})$.

\section{On OUTPUT}

ZR and ZI contain the real and imaginary parts, respectively, of the transformed eigenvectors

C respectively, of the trans

C

C Questions and comments should be directed to B. S. Garbow, C APPLIED MATHEMATICS DIVISION, ARGONNE NATIONAL LABORATORY C C***REFERENCES B.T. SMITH, J.M. BOYLE, J.J. DONGARRA, B.S. GARBOW, C Y. IKEBE, V. C. KLEMA, C. B. MOLER, *MATRIX EIGEN-

C SYSTEM ROUTINES - EISPACK GUIDE*, SPRINGER-VERLAG, C 1976.

C***ROUTINES CALLED (NONE)

$C^{* * *}$ END PROLOGUE CBABK2

C

INTEGER I,J,K,M,N,II,NM,IGH,LOW

REAL*8 SCALE $(600), Z R(600,600), Z I(600,600)$

REAL*8 S 


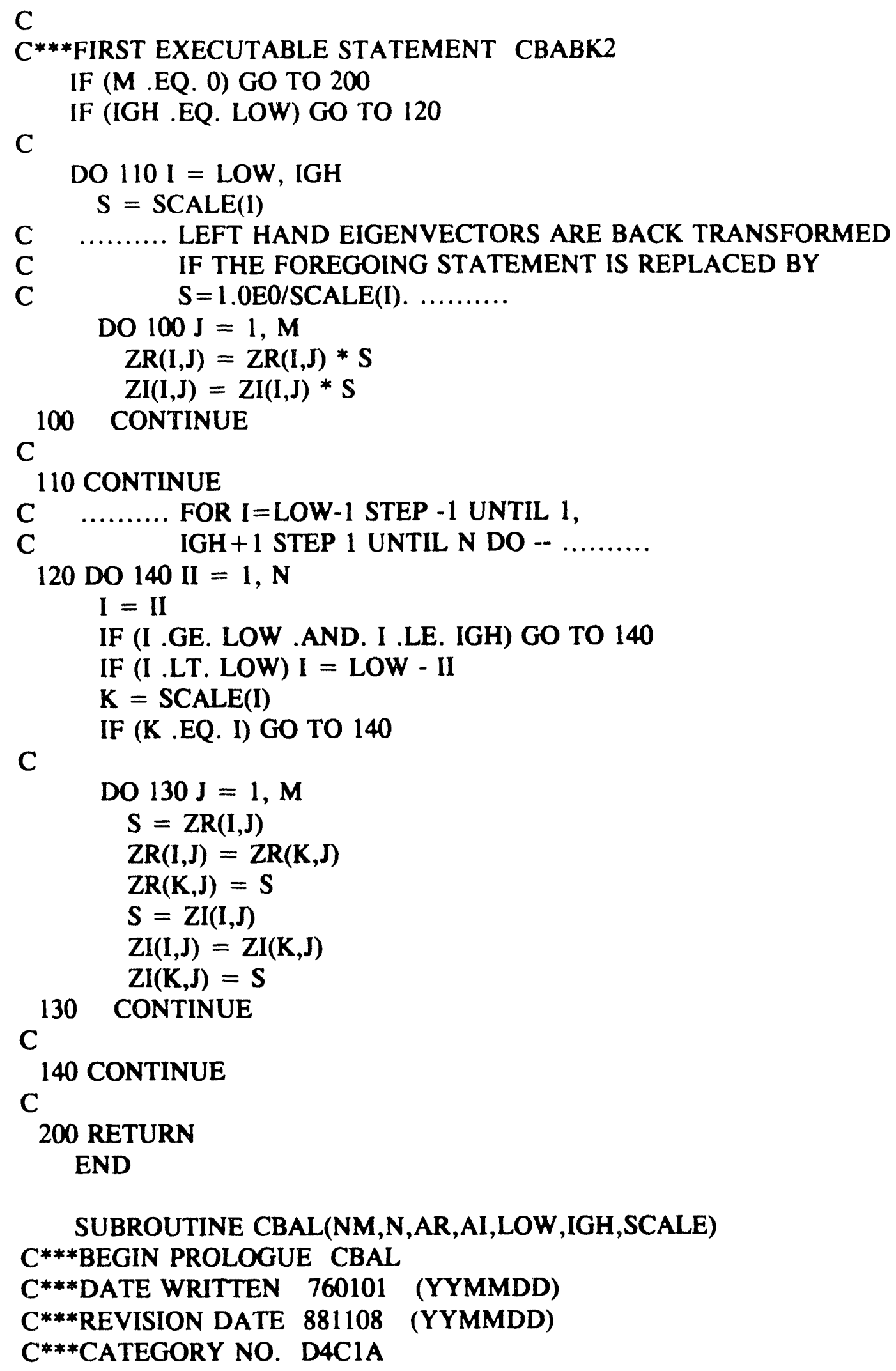







C Suppose that the principal submatrix in rows LOW through IGH

$C$ has been baianced, that $P(J)$ denotes the index interchanged

$C$ with $J$ during the permutation step, and that the elements

$C$ of the diagonal matrix used are denoted by $D(I, J)$. Then

C $\quad \operatorname{SCALE}(\mathrm{J})=\mathrm{P}(\mathrm{J})$, for $\mathrm{J}=1, \ldots$, LOW -1

$\mathrm{C} \quad=\mathrm{D}(\mathrm{J}, \mathrm{J}) \quad \mathrm{J}=\mathrm{LOW}, \ldots, \mathrm{IGH}$

$\mathrm{C} \quad=\mathrm{P}(\mathrm{J}) \quad \mathrm{J}=\mathrm{IGH}+1, \ldots, \mathrm{N}$.

C The order in which the interchanges are made is $\mathbf{N}$ to $\mathrm{IGH}+1$,

C then 1 to LOW-1.

C

C Note that 1 is returned for IGH if IGH is zero formally.

C

C The ALGOL procedure EXC contained in CBALANCE appears in

$\mathrm{C}$ CBAL in line. (Note that the ALGOL roles of identifiers

C $K, L$ have been reversed.)

C

C Questions and comments should be directed to B. S. Garbow,

C APPLIED MATHEMATICS DIVISION, ARGONNE NATIONAL LABORATORY

C

C***REFERENCES B.T. SMITH, J.M. BOYLE, J.J. DONGARRA, B.S. GARBOW, C Y. IKEBE, V. C. KLEMA, C. B. MOLER, *MATRIX EIGEN-

C SYSTEM ROUTINES - EISPACK GUIDE*, SPRINGER-VERLAG, C 1976.

¿**ROUTINES CALLED (NONE)

$C^{* * *}$ END PROLOGUE CBAL

$\mathrm{C}$

INTEGER I,J,K,L,M,N,JJ,NM,IGH,LOW,IEXC

REAL*8 AR(600,600),AI(600,600),SCALE(600)

REAL*8 C,F,G,R,S,B2,RADIX

C

LOGICAL NOCONV

C THE FOLLOWING PORTABLE VALUE OF RADIX WORKS WELL ENOUGH

C FOR ALL MACHINES WHOSE BASE IS A POWER OF TWO.

C

C***FIRST EXECUTABLE STATEMENT CBAL

RADIX $=16$

C

$\mathrm{B2}=\mathrm{RADIX} *$ RADIX

$\mathrm{K}=1$

$\mathbf{L}=\mathbf{N}$

GO TO 100

C $\quad \ldots \ldots \ldots$ IN-LINE PROCEDURE FOR ROW AND

C COLUMN EXCHANGE

$20 \operatorname{SCALE}(\mathrm{M})=\mathrm{J}$ 
IF (J .EQ. M) GO TO 50

C

DO $30 \mathrm{I}=1, \mathrm{~L}$

$F=A R(I, J)$

$\operatorname{AR}(I, J)=A R(I, M)$

$A R(I, M)=F$

$\mathrm{F}=\mathrm{AI}(\mathrm{I}, \mathrm{J})$

$\mathrm{AI}(\mathrm{I}, \mathrm{J})=\mathrm{AI}(\mathrm{I}, \mathrm{M})$

$\mathrm{AI}(\mathrm{I}, \mathrm{M})=\mathrm{F}$

30 CONTINUE

C

DO $40 \mathrm{I}=\mathrm{K}, \mathrm{N}$

$\mathrm{F}=\mathrm{AR}(\mathrm{J}, \mathrm{I})$

$A R(J, I)=A R(M, I)$

$\operatorname{AR}(\mathbf{M}, \mathbf{I})=\mathrm{F}$

$\mathrm{F}=\mathrm{AI}(\mathrm{J}, \mathrm{I})$

$\operatorname{AI}(\mathrm{J}, \mathrm{I})=\mathrm{AI}(\mathrm{M}, \mathrm{I})$

$\mathrm{AI}(\mathrm{M}, \mathrm{I})=\mathrm{F}$

40 CONTINUE

C

50 GO TO $(80,130)$, IEXC

C ......... SEARCH FOR ROWS ISOLATING AN EIGENVALUE

C AND PUSH THEM DOWN

80 IF (L .EQ. 1) GO TO 280

$\mathrm{L}=\mathrm{L}-1$

C FOR J = L STEP -1 UNTIL 1 DO -. .........

$100 \mathrm{DO} 120 \mathrm{JJ}=1, \mathrm{~L}$

C

$$
\mathrm{J}=\mathrm{L}+1-\mathrm{J} \mathrm{J}
$$

DO $110 \mathrm{I}=1, \mathrm{~L}$

IF (I .EQ. J) GO TO 110

IF (AR(J,I) .NE. 0.0EO .OR. AI(J,I) .NE. 0.0E0) GO TO 120

110 CONTINUE

C

$\mathbf{M}=\mathbf{L}$

IEXC $=1$

GO TO 20

120 CONTINUE

C

GO TO 140

C ........ SEARCH FOR COLUMNS ISOLATING AN EIGENVALUE

C AND PUSH THEM LEFT ..........

$$
130 K=K+1
$$

C 
$140 \mathrm{DO} 170 \mathrm{~J}=\mathrm{K}, \mathrm{L}$

C

DO $150 \mathrm{I}=\mathrm{K}, \mathrm{L}$

IF (I .EQ. J) GO TO 150

IF (AR(I,J) .NE. 0.0E0 .OR. AI(I,J) .NE. 0.0E0) GO TO 170

150 CONTINUE

C

$\mathbf{M}=\mathbf{K}$

IEXC $=2$

GO TO 20

170 CONTINUE

C ........ NOW BALANCE THE SUBMATRIX IN ROWS K TO L ........

DO $180 \mathrm{I}=\mathrm{K}, \mathrm{L}$

$180 \operatorname{SCALE}(\mathrm{I})=1.0 \mathrm{E} 0$

C ........ ITERATIVE LOOP FOR NORM REDUCTION .........

190 NOCONV $=$.FALSE.

C

DO $270 \mathrm{I}=\mathrm{K}, \mathrm{L}$

$\mathrm{C}=0.0 \mathrm{E} 0$

C

$\mathrm{R}=0.0 \mathrm{E} 0$

DO $200 \mathrm{~J}=\mathrm{K}, \mathrm{L}$

IF (J .EQ. I) GO TO 200

$C=C+A B S(A R(J, I))+A B S(A I(J, I))$

$R=R+A B S(A R(I, J))+A B S(A I(I, J))$

200 CONTINUE

C ......... GUARD AGAINST ZERO C OR R DUE TO UNDERFLOW

IF (C .EQ. 0.0EO .OR. R .EQ. 0.0E0) GO TO 270

$\mathbf{G}=\mathbf{R} / \mathbf{R A D I X}$

$F=1.0 \mathrm{E} 0$

$\mathbf{S}=\mathbf{C}+\mathbf{R}$

210 IF (C .GE. G) GO TO 220

$\mathbf{F}=\mathbf{F} * \mathbf{R A D I X}$

$\mathrm{C}=\mathrm{C} * \mathrm{~B} 2$

GO TO 210

$220 \mathrm{G}=\mathrm{R} * \mathrm{RADIX}$

230 IF (C .LT. G) GO TO 240

$F=F / R A D I X$

$\mathrm{C}=\mathrm{C} / \mathrm{B} 2$

GO TO 230

C ......... NOW BALANCE

240 IF $((C+R) / F . G E .0 .95 E 0 * S)$ GO TO 270

$\mathrm{G}=1.0 \mathrm{E} 0 / \mathrm{F}$

SCALE $(I)=$ SCALE $(I) * F$ 


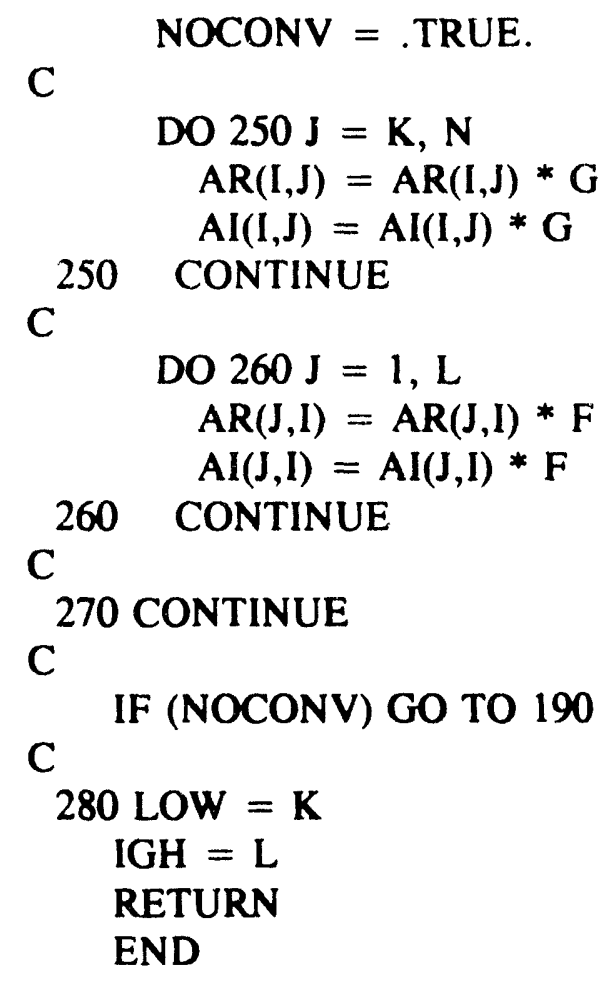

SUBROUTINE CDIV(AR,AI,BR,BI,CR,CI)

$C^{* * * B E G I N ~ P R O L O G U E ~ C D I V ~}$

$C^{* * *}$ REFER TO EISDOC

C

C Complex division, $(\mathrm{CR}, \mathrm{CI})=(\mathrm{AR}, \mathrm{AI}) /(\mathrm{BR}, \mathrm{BI})$

$C^{* * * \text { ROUTINES CALLED (NONE) }}$

C***END PROLOGUE CDIV

C

$\mathrm{REAL} * 8 \mathrm{AR}, \mathrm{AI}, \mathrm{BR}, \mathrm{BI}, \mathrm{CR}, \mathrm{CI}$

REAL*8 S,ARS,AIS,BRS,BIS

C***FIRST EXECUTABLE STATEMENT CDIV

$S=A B S(B R)+A B S(B I)$

ARS $=$ AR/S

AIS $=$ AI/S

$\mathrm{BRS}=\mathrm{BR} / \mathrm{S}$

$\mathrm{BIS}=\mathrm{BI} / \mathrm{S}$

$\mathrm{S}=\mathrm{BRS}^{* * 2}+\mathrm{BIS} * * 2$

$\mathrm{CR}=(\mathrm{ARS} * \mathrm{BRS}+\mathrm{AIS} * \mathrm{BIS}) / \mathrm{S}$

$\mathrm{CI}=(\mathrm{AIS} * \mathrm{BRS}-\mathrm{ARS} * \mathrm{BIS}) / \mathrm{S}$

RETURN

END

SUBROUTINE COM2(NM,N,LOW,IGH,ORTR,ORTI,HR,HI,WR,WI,ZR,ZI,IERR) 





C one-dimensional REAL arrays, dimensioned ORTR(IGH) and

C ORTI(IGH).

C

C HR and HI contain the real and imaginary parts, respectively,

$\mathrm{C}$ of the complex upper Hessenberg matrix. Their lower

C triangles below the subdiagonal contain information about

C the unitary transformations used in the reduction by CORTH,

$\mathrm{C}$ if performed. If the eigenvectors of the Hessenberg matrix

C are desired, these elements may be arbitrary. HR and $\mathrm{HI}$

C are two-dimensional REAL arrays, dimensioned HR(NM,N) and

C HI(NM,N).

C

C On OUTPUT

C

C ORTR, ORTI, and the upper Hessenberg portions of HR and HI

C have been destroyed.

C

C WR and WI contain the real and imaginary parts, respectively,

C of the eigenvalues of the upper Hessenberg matrix. If an

C error exit is made, the eigenvalues should be correct for

$C$ indices IERR +1, IERR +2, .., N. WR and WI are one-

C dimensional REAL arrays, dimensioned WR(N) and WI(N).

C

C ZR and $\mathrm{ZI}$ contain the real and imaginary parts, respectively,

C of the eigenvectors. The eigenvectors are unnormalized.

C If an error exit is made, none of the eigenvectors has been

$C$ found. ZR and $\mathrm{ZI}$ are two-dimensional REAL arrays,

$C$ dimensioned $\mathrm{ZR}(\mathrm{NM}, \mathrm{N})$ and $\mathrm{ZI}(\mathrm{NM}, \mathrm{N})$.

C

C

IERR is an INTEGER flag set to

Zero for normal return,

$\mathrm{J}$ if the J-th eigenvalue has not been

determined after a total of $30 * \mathrm{~N}$ iterations.

The eigenvalues should be correct for indices

IERR + 1, IERR + 2, .., N, but no eigenvectors are computed.

Calls CSROOT for complex square root.

C Calls PYTHAG(A,B) for $\operatorname{sqrt}\left(A^{* * 2}+B^{* * 2}\right)$.

C Calls CDIV for complex division.

C

C Questions and comments should be directed to B. S. Garbow,

C APPLIED MATHEMATICS DIVISION, ARGONNE NATIONAL LABORATORY

C 
C***REFERENCES B.T. SMITH, J.M. BOYLE, J.J. DONGARRA, B.S. GARBOW,

C Y. IKEBE, V. C. KLEMA, C. B. MOLER, *MATRIX EIGEN-

C SYSTEM ROUTINES - EISPACK GUIDE*, SPRINGER-VERLAG,

C 1976.

C***ROUTINES CALLED CDIV,CSROOT,PYTHAG

$C^{* * * E N D ~ P R O L O G U E ~ C O M Q R 2 ~}$

C

C

$\mathrm{C}$

C Change record:

C 89-05-31 Changed all specific intrinsics to generic. (WRB)

C

C

C

C

INTEGER I,J,K,L,M,N,EN,II,JJ,LL,NM,NN,IGH,IP1

INTEGER ITN,ITS, LOW, LPI,ENM I,IEND,IERR

REAL*8 HR(600,600), HI $(600,600), W R(600), W I(600)$

REAL*8 ZR(600,600),ZI(600,600), ORTR(600), ORTI(600)

REAL *8 SI,SR, TI,TR,XI,XR, YI, YR,ZZI,ZZR, NORM,S1,S2

REAL*8 PYTHAG

C

C***FIRST EXECUTABLE STATEMENT COMQR2

IERR $=0$

C

DO $100 \mathrm{I}=1, \mathrm{~N}$

C

$$
\begin{aligned}
& \text { DO } 100 \mathrm{~J}=1, \mathrm{~N} \\
& \mathrm{ZR}(\mathrm{I}, \mathrm{J})=0.0 \mathrm{E} 0 \\
& \mathrm{ZI}(\mathrm{I}, \mathrm{J})=0.0 \mathrm{E} 0 \\
& \mathrm{IF}(\mathrm{I} . \mathrm{EQ} . \mathrm{J}) \mathrm{ZR}(\mathrm{I}, \mathrm{J})=1.0 \mathrm{E} 0
\end{aligned}
$$

100 CONTINUE

C ........ FORM THE MATRIX OF ACCUMULATED TRANSFORMATIONS

C FROM THE INFORMATION LEFT BY CORTH

IEND $=$ IGH - LOW -1

IF (IEND) 180, 150, 105

C $\quad \ldots \ldots \ldots$ FOR I =IGH-1 STEP -1 UNTIL LOW +1 DO..$- \ldots \ldots \ldots$

105 DO $140 \mathrm{II}=1$, IEND

$\mathrm{I}=\mathrm{IGH}-\mathrm{II}$

IF (ORTR(I) .EQ. 0.0E0 .AND. ORTI(I). EQ. 0.0E0) GO TO 140

IF (HR(I,I-1).EQ.0.0E0 .AND. HI(I,I-1).EQ.0.0E0) GO TO 140

C ......... NORM BELOW IS NEGATIVE OF H FORMED IN CORTH

NORM $=$ HR(I,I-1) * ORTR(I) + HI(I,I-1) * ORTI(I)

IPI $=1+1$ 
C

110

$$
\begin{aligned}
& \text { DO } 110 \mathrm{~K}=\mathrm{IP} 1, \mathrm{IGH} \\
& \text { ORTR(K) }=\mathrm{HR}(\mathrm{K}, \mathrm{I}-1) \\
& \text { ORTI }(\mathrm{K})=\mathrm{HI}(\mathrm{K}, \mathrm{I}-1)
\end{aligned}
$$

C

$$
\text { CONTINUE }
$$

DO $130 \mathrm{~J}=\mathrm{I}, \mathrm{IGH}$

$$
\begin{aligned}
& \mathrm{SR}=0.0 \mathrm{E} 0 \\
& \mathrm{SI}=0.0 \mathrm{EO}
\end{aligned}
$$

C

$$
\begin{aligned}
& \text { DO } 115 \mathrm{~K}=\mathrm{I}, \mathrm{IGH} \\
& \mathrm{SR}=\mathrm{SR}+\mathrm{ORTR}(\mathrm{K}) * \mathrm{ZR}(\mathrm{K}, \mathrm{J})+\mathrm{ORTI}(\mathrm{K}) * \mathrm{ZI}(\mathrm{K}, \mathrm{J}) \\
& \mathrm{SI}=\mathrm{SI}+\mathrm{ORTR}(\mathrm{K}) * \mathrm{ZI}(\mathrm{K}, \mathrm{J})-\mathrm{ORTI}(\mathrm{K}) * \mathrm{ZR}(\mathrm{K}, \mathrm{J})
\end{aligned}
$$

115 CONTINUE

C

$$
\begin{aligned}
& \mathrm{SR}=\mathrm{SR} / \mathrm{NORM} \\
& \mathrm{SI}=\mathrm{SI} / \mathrm{NORM}
\end{aligned}
$$

C

DO $120 \mathrm{~K}=\mathrm{I}, \mathrm{IGH}$

$$
\begin{aligned}
& \mathrm{ZR}(\mathrm{K}, \mathrm{J})=\mathrm{ZR}(\mathrm{K}, \mathrm{J})+\mathrm{SR} * \text { ORTR(K) }-\mathrm{SI} * \text { ORTI(K) } \\
& \mathrm{ZI}(\mathrm{K}, \mathrm{J})=\mathrm{ZI}(\mathrm{K}, \mathrm{J})+\mathrm{SR} * \text { ORTI(K) }+\mathrm{SI} * \text { ORTR(K) }
\end{aligned}
$$

120 CONTINUE

C

130 CONTINUE

C.

140 CONTINUE

C ........ CREATE REAL SUBDIAGONAL ELEMENTS .........

$150 \mathrm{~L}=\mathrm{LOW}+1$

C

DO $170 \mathrm{I}=\mathrm{L}, \mathrm{IGH}$

$\mathrm{LL}=\mathrm{MIN}(\mathrm{I}+1, \mathrm{IGH})$

IF (HI(I,I-1) .EQ. 0.0E0) GO TO 170

NORM $=$ PYTHAG(HR(I,I-1),HI(I,I-1))

$Y R=H R(I, I-1) / N O R M$

$Y I=H I(I, I-1) / N O R M$

$\operatorname{HR}(I, I-1)=$ NORM

C

$\mathrm{HI}(\mathrm{I}, \mathrm{I}-1)=0.0 \mathrm{E} 0$

DO $155 \mathrm{~J}=\mathrm{I}, \mathrm{N}$

$$
\begin{aligned}
& \mathrm{SI}=\mathrm{YR} * \mathrm{HI}(\mathrm{I}, \mathrm{J})-\mathrm{YI} * \mathrm{HR}(\mathrm{I}, \mathrm{J}) \\
& \mathrm{HR}(\mathrm{I}, \mathrm{J})=\mathrm{YR} * \mathrm{HR}(\mathrm{I}, \mathrm{J})+\mathrm{YI} * \mathrm{HI}(\mathrm{I}, \mathrm{J}) \\
& \mathrm{HI}(\mathrm{I}, \mathrm{J})=\mathrm{SI}
\end{aligned}
$$

155 CONTINUE

C 


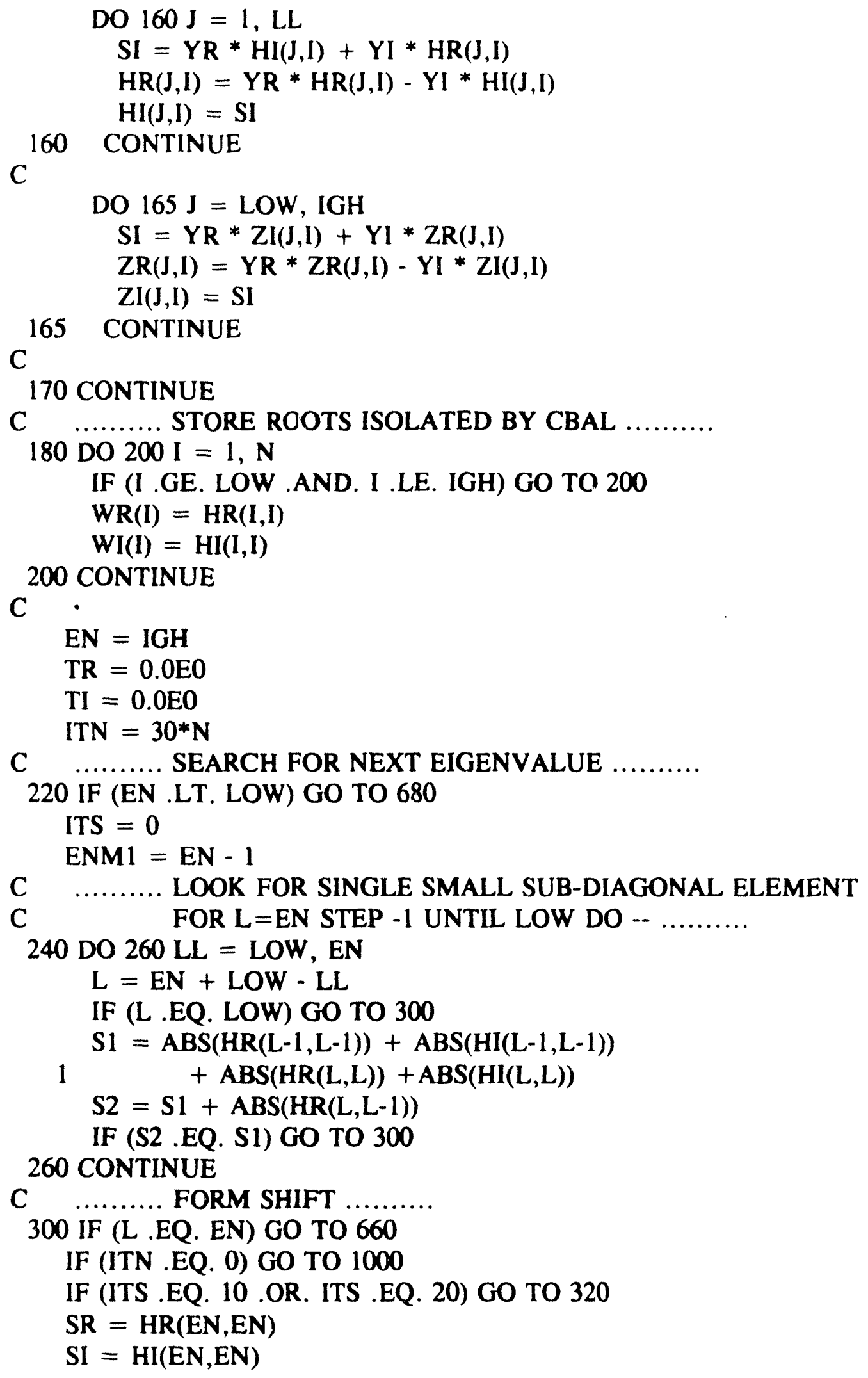




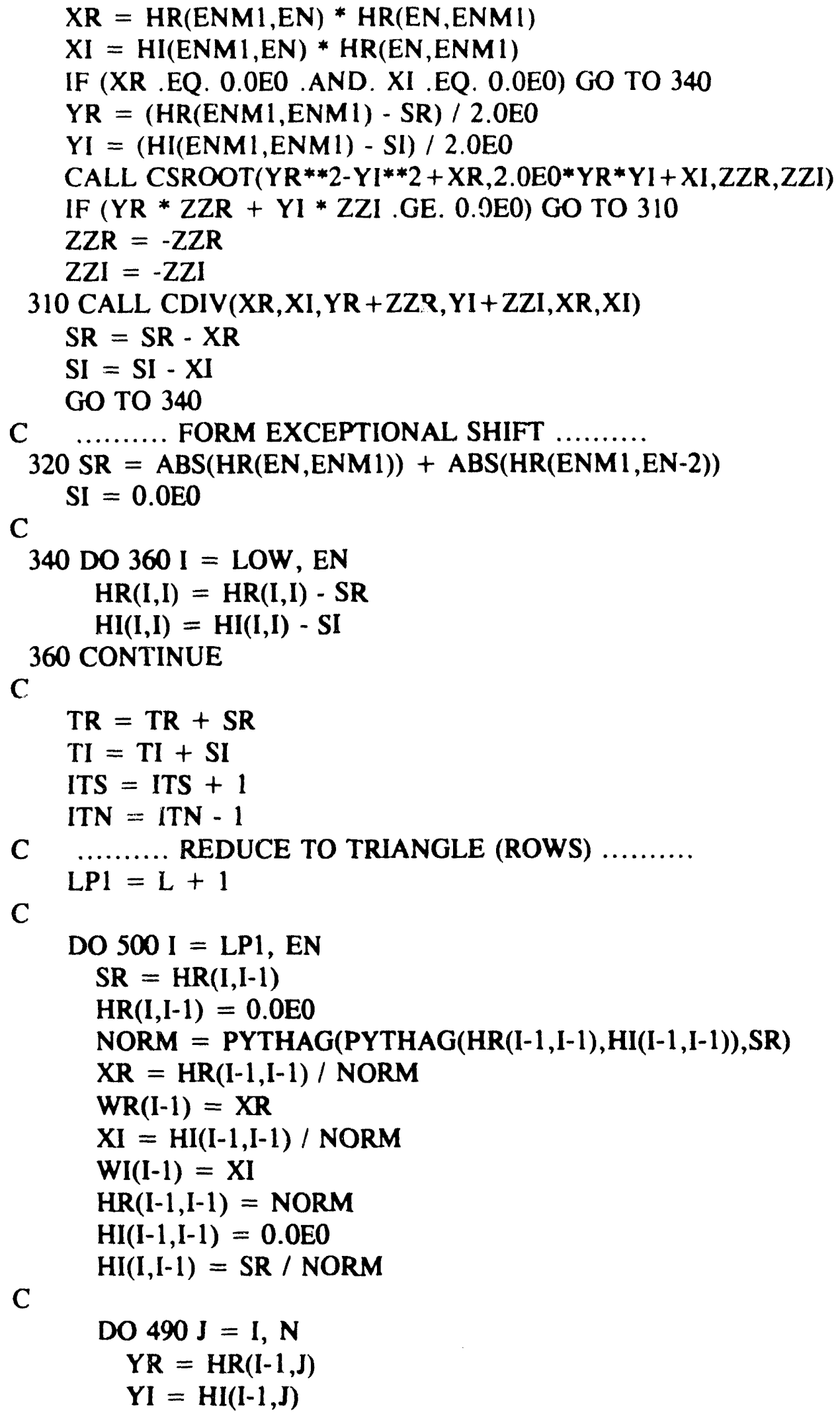






C

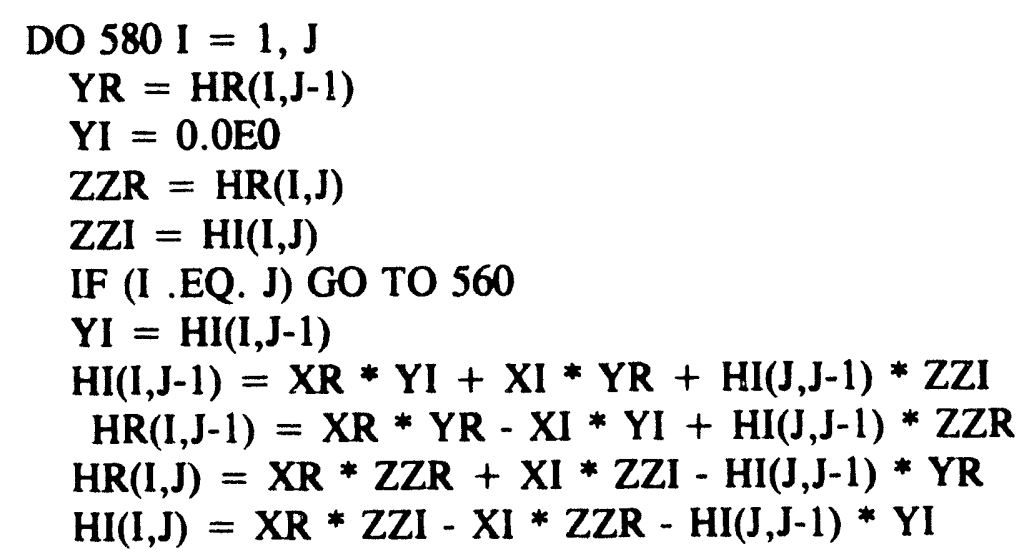

580 CONTINUE

C 







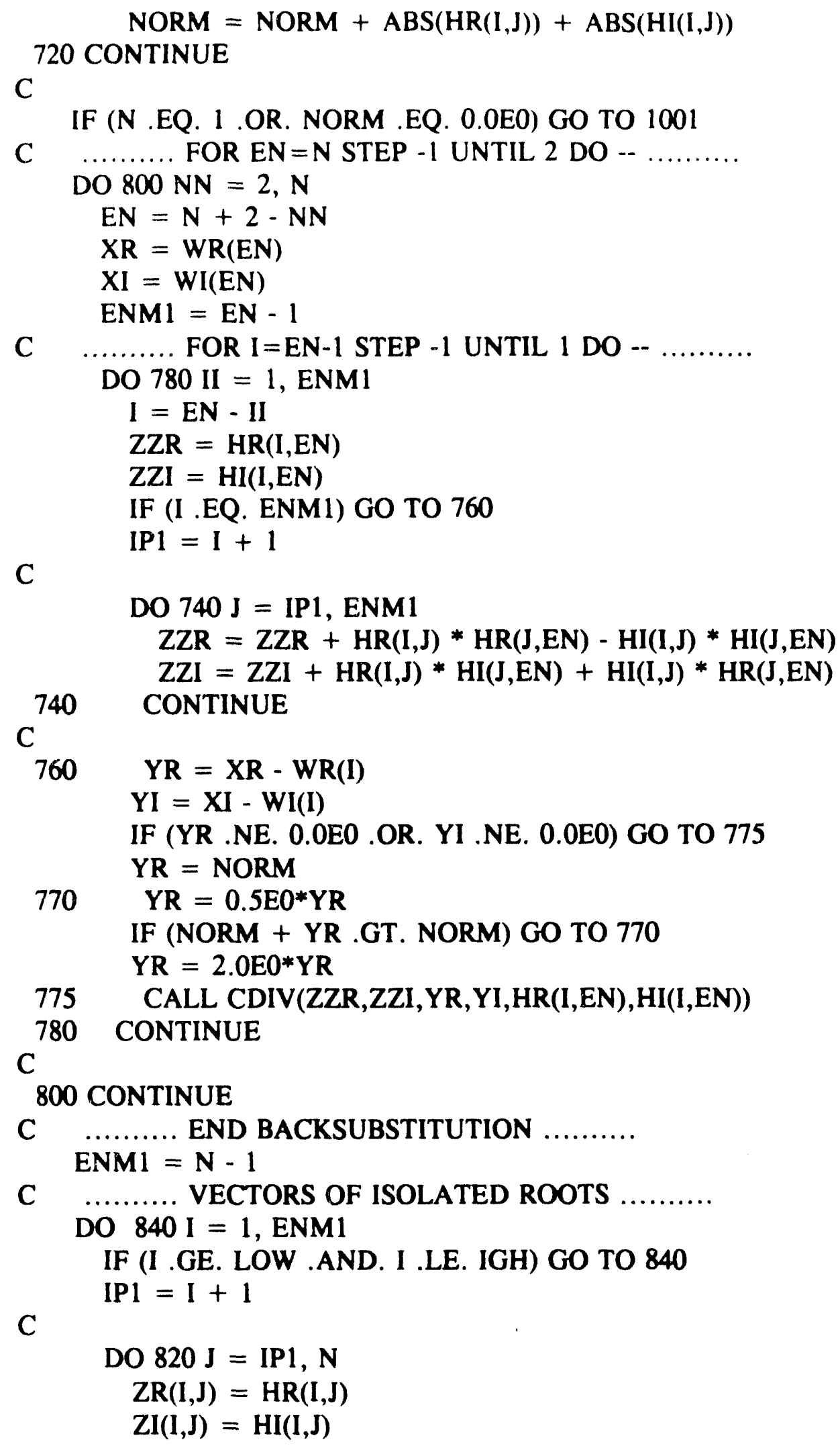




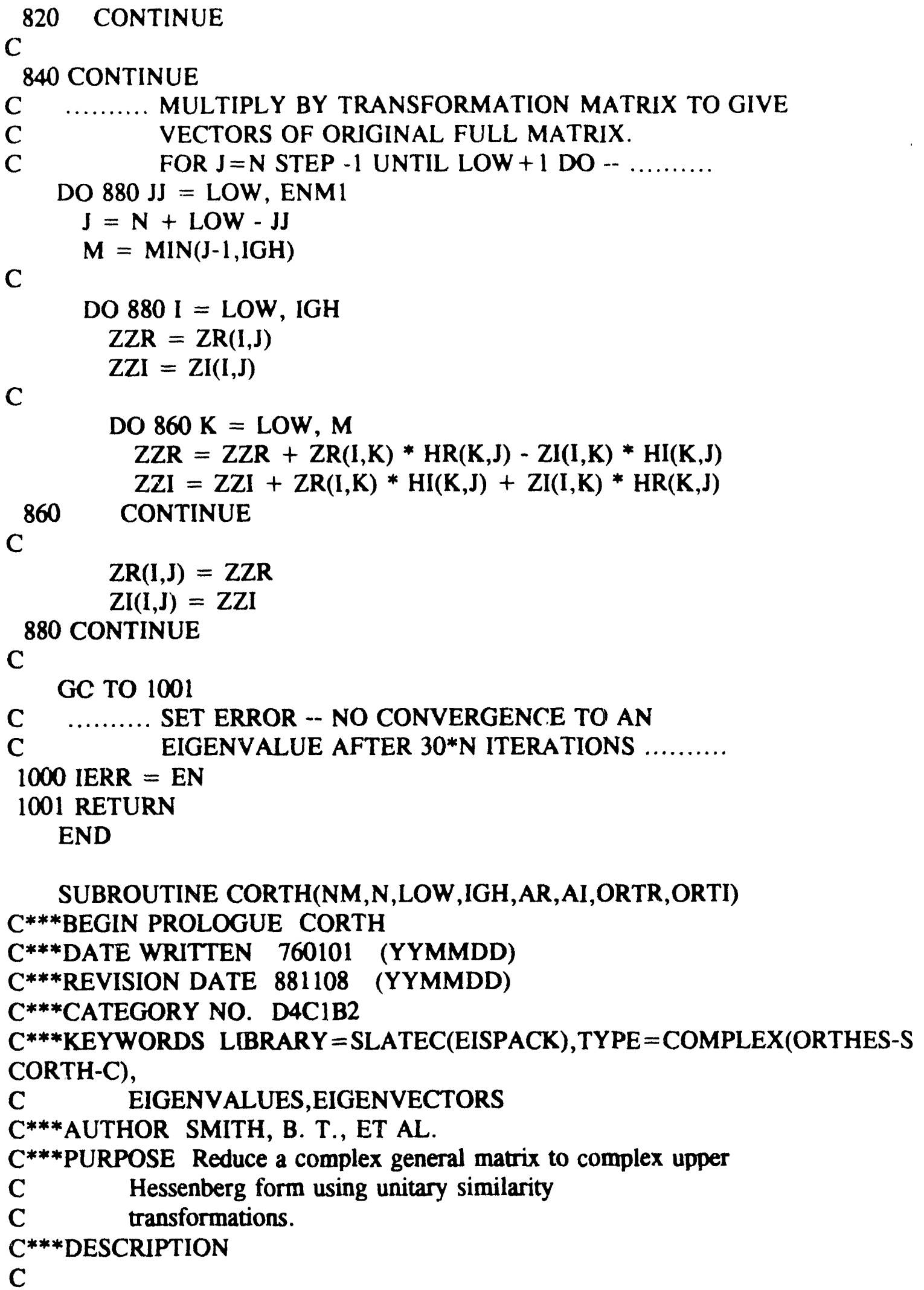


C This subroutine is a translation of a complex analogue of

C the ALGOL procedure ORTHES, NUM. MATH. 12, 349-368(1968)

C by Martin and Wilkinson.

C HANDBOOK FOR AUTO. COMP., VOL.II-LINEAR ALGEBRA, 339-358(1971).

C

Given a COMPLEX GENERAL matrix, this subroutine reduces a submatrix situated in rows and columns

C LOW through IGH to upper Hessenberg form by

C unitary similarity transformations.

C

C

On INPUT

NM must be set to the row dimension of the two-dimensional array parameters, $\mathrm{AR}$ and $\mathrm{Al}$, as declared in the calling program dimension statement. NM is an INTEGER variable.

$\mathbf{N}$ is the order of the matrix $A=(A R, A I) . N$ is an INTEGER variable. $\mathbf{N}$ must be less than or equal to $\mathrm{NM}$.

LOW and IGH are two INTEGER variables determined by the balancing subroutine CBAL. If CBAL has not been used, set LOW $=1$ and IGH equal to the order of the matrix, $N$.

$A R$ and $A I$ contain the real and imaginary parts, respectively, of the complex input matrix. $A R$ and $A I$ are two-dimensional REAL arrays, dimensioned $A R(N M, N)$ and $A I(N M, N)$.

\section{On OUTPUT}

C

$A R$ and $A I$ contain the real and imaginary parts, repectively, of the Hessenberg matrix. Information about the unitary transformations used in the reduction is stored in the remaining triangles under the Hessenberg matrix.

ORTR and ORTI contain further information about the unitary transformations. Only elements LOW through IGH are used. ORTR and ORTI are one-dimensional REAL arrays, dimensioned C ORTR(IGH) and ORTI(IGH).

C

C

C Questions and comments should be directed to B. S. Garbow, C APPLIED MATHEMATICS DIVISION, ARGONNE NATIONAL LABORATORY

C 
C***REFERENCES B.T. SMITH, J.M. BOYLE, J.J. DONGARRA, B.S. GARBOW, C Y. IKEBE, Y. C. KLEMA, C. B. MOLER, *MATRIX EIGEN-

C SYSTEM ROUTINES - EISPACK GUIDE*, SPRINGER-VERLAG, C 1976.

C***ROUTINES CALLED PYTHAG

C***END PROLOGUE CORTH

C

INTEGER I,J,M,N,II,JJ,LA,MP,NM,IGH,KP1,LOW

REAL*8 AR(600,600),AI $(600,600)$, ORTR $(600), \operatorname{ORTI}(600)$

REAL*8 F,G,H,FI,FR,SCALE

C

REAL*8 PYTHAG

C***FIRST EXECUTABLE STATEMENT CORTH

LA $=$ IGH -1

$\mathrm{KP1}=\mathrm{LOW}+1$

IF (LA .LT. KP1) GO TO 200

C

DO $180 \mathrm{M}=\mathrm{KP1}, \mathrm{LA}$

$\mathrm{H}=0.0 \mathrm{E} 0$

$\operatorname{ORTR}(\mathrm{M})=0.0 \mathrm{E} 0$

$\operatorname{ORTI}(M)=0.0 \mathrm{EO}$

SCALE $=0.0 \mathrm{E} 0$

C $\ldots \ldots \ldots$. SCALE COLUMN (ALGOL TOL THEN NOT NEEDED) .......... DO $90 \mathrm{I}=\mathrm{M}, \mathrm{IGH}$

$\mathrm{C}$

90 SCALE $=$ SCALE + ABS(AR(I,M-1)) + ABS(AI(I,M-1))

IF (SCALE .EQ. 0.0E0) GO TO 180

$\mathbf{M P}=\mathbf{M}+\mathbf{I G H}$

C $\quad \ldots \ldots \ldots$ FOR I $=$ IGH STEP -1 UNTIL M DO -.........

DO $100 \mathrm{II}=\mathrm{M}, \mathrm{IGH}$

$\mathrm{I}=\mathrm{MP}-\mathrm{II}$

ORTR(I) $=$ AR(I,M-1) $/$ SCALE

ORTI(I) $=$ AI $(I, M-1) /$ SCALE

$\mathrm{H}=\mathrm{H}+\mathrm{ORTR}(\mathrm{I}) * \mathrm{ORTR}(\mathrm{I})+\mathrm{ORTI}(\mathrm{I}) * \mathrm{ORTI}(\mathrm{I})$

100 CONTINUE

$\mathrm{C}$

$\mathrm{G}=\mathrm{SQRT}(\mathrm{H})$

$\mathrm{F}=$ PYTHAG(ORTR(M),ORTI(M))

IF (F .EQ. 0.0E0) GO TO 103

$\mathbf{H}=\mathbf{H}+\mathbf{F}^{*} \mathbf{G}$

$\mathbf{G}=\mathbf{G} / \mathbf{F}$

$\operatorname{ORTR}(M)=(1.0 \mathrm{E} 0+\mathrm{G}) * \operatorname{ORTR}(\mathrm{M})$

ORTI(M) $=(1.0 \mathrm{EO}+\mathrm{G}) *$ ORTI(M)

GO TO 105 
C

103 ORTR(M) $=\mathrm{G}$

$\operatorname{AR}(M, M-1)=$ SCALE

C $\quad \ldots \ldots \ldots$ FORM $(\mathrm{I}-(\mathrm{U} * \mathrm{UT}) / \mathrm{H}) *$ A $\ldots \ldots \ldots$

105 DO $130 \mathrm{~J}=\mathrm{M}, \mathrm{N}$

$\mathrm{FR}=0.0 \mathrm{EO}$

$\mathrm{FI}=0.0 \mathrm{E} 0$

C $\quad \ldots \ldots \ldots$ FOR I $=$ IGH STEP -1 UNTIL $M$ DO...$- \ldots \ldots$

DO 110 II $=$ M, IGH

$I=$ MP - II

$\mathrm{FR}=\mathrm{FR}+\mathrm{ORTR}(\mathrm{I}) * \mathrm{AR}(\mathrm{I}, \mathrm{J})+\mathrm{ORTI}(\mathrm{I}) * \mathrm{AI}(\mathrm{I}, \mathrm{J})$

$\mathrm{FI}=\mathrm{FI}+\mathrm{ORTR}(\mathrm{I}) * \mathrm{AI}(\mathrm{I}, \mathrm{J})-\mathrm{ORTI}(\mathrm{I}) * \mathrm{AR}(\mathrm{I}, \mathrm{J})$

110 CONTINUE

C

$\mathrm{FR}=\mathrm{FR} / \mathrm{H}$

$\mathrm{FI}=\mathrm{FI} / \mathrm{H}$

C

DO $120 \mathrm{I}=\mathrm{M}, \mathrm{IGH}$

$\operatorname{AR}(I, J)=A R(I, J)-F R *$ ORTR(I) + FI * ORTI(I)

$A I(I, J)=A I(I, J)-F R *$ ORTI(I) - FI * ORTR(I)

120 CONTINUE

$\mathrm{C}$

130 CONTINUE

C $\quad \ldots \ldots \ldots$ FORM $\left(\mathrm{I}-\left(\mathrm{U}^{*} \mathrm{UT}\right) / \mathrm{H}\right)^{*} \mathrm{~A}^{*}\left(\mathrm{I}-\left(\mathrm{U}^{*} \mathrm{UT}\right) / \mathrm{H}\right) \ldots \ldots \ldots$

DO $160 \mathrm{I}=1$, IGH

$\mathrm{FR}=0.0 \mathrm{EO}$

$\mathrm{FI}=0.0 \mathrm{E} 0$

C $\quad \ldots \ldots \ldots .$. FOR J =IGH STEP -1 UNTIL $M$ DO -. ........

DO $140 \mathrm{JJ}=\mathrm{M}, \mathrm{IGH}$

$\mathbf{J}=\mathbf{M P}-\mathbf{J J}$

$\mathrm{FR}=\mathrm{FR}+\mathrm{ORTR}(\mathrm{J})$ *AR(I,J) - ORTI(J) * AI(I,J)

$\mathrm{FI}=\mathrm{FI}+\mathrm{ORTR}(\mathrm{J}) * \mathrm{AI}(\mathrm{I}, \mathrm{J})+\mathrm{ORTI}(\mathrm{J}) * \mathrm{AR}(\mathrm{I}, \mathrm{J})$

140 CONTINUE

C

$\mathbf{F R}=\mathbf{F R} / \mathbf{H}$

$\mathrm{FI}=\mathrm{FI} / \mathrm{H}$

C

DO $150 \mathrm{~J}=\mathrm{M}, \mathrm{IGH}$

$A R(I, J)=A R(I, J)-F R *$ ORTR(J) - FI * ORTI(J)

$A I(I, J)=A I(I, J)+F R *$ ORTI(J) - FI * ORTR(J)

150 CONTINUE

C

160 CONTINUE

C 


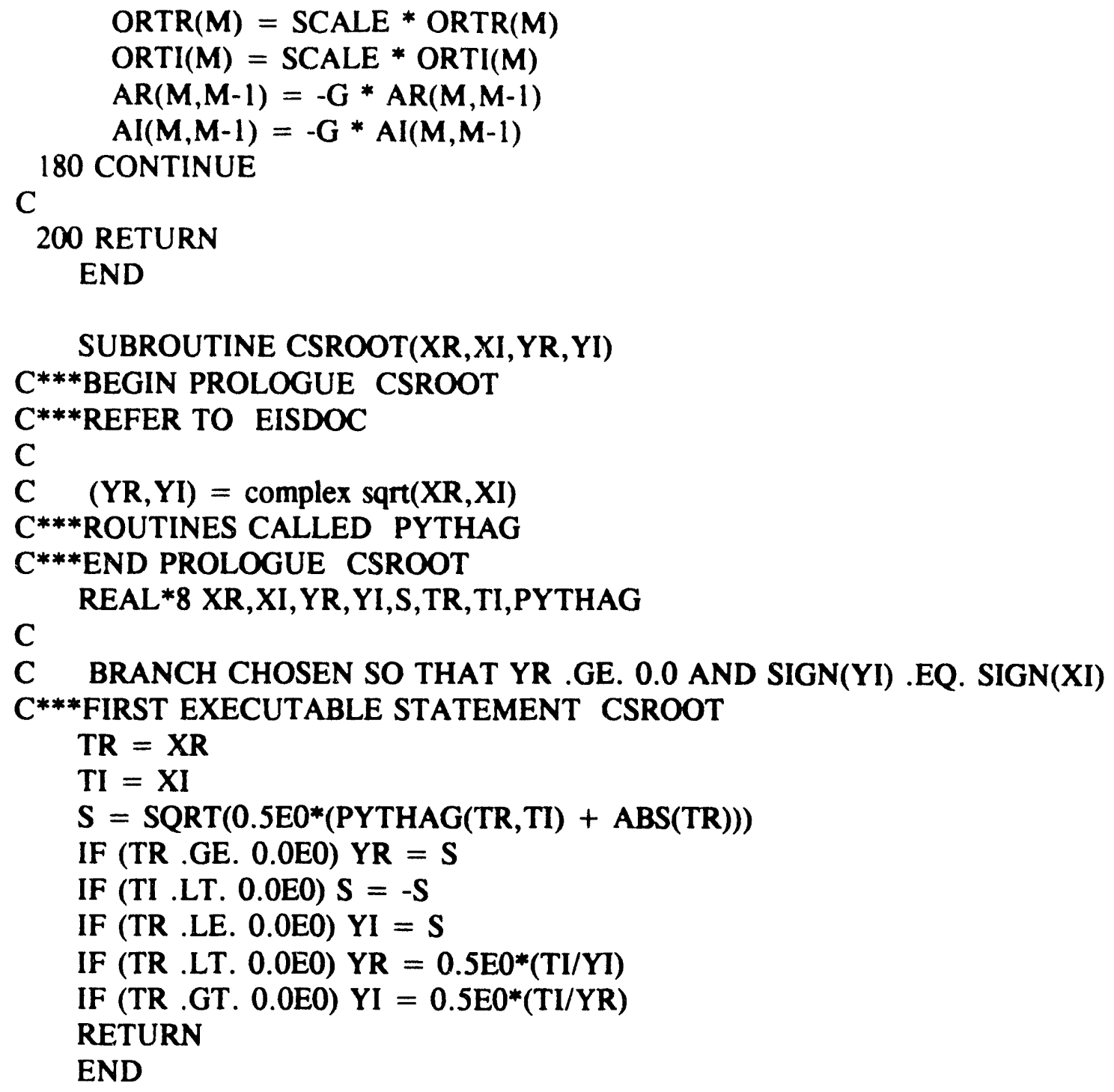




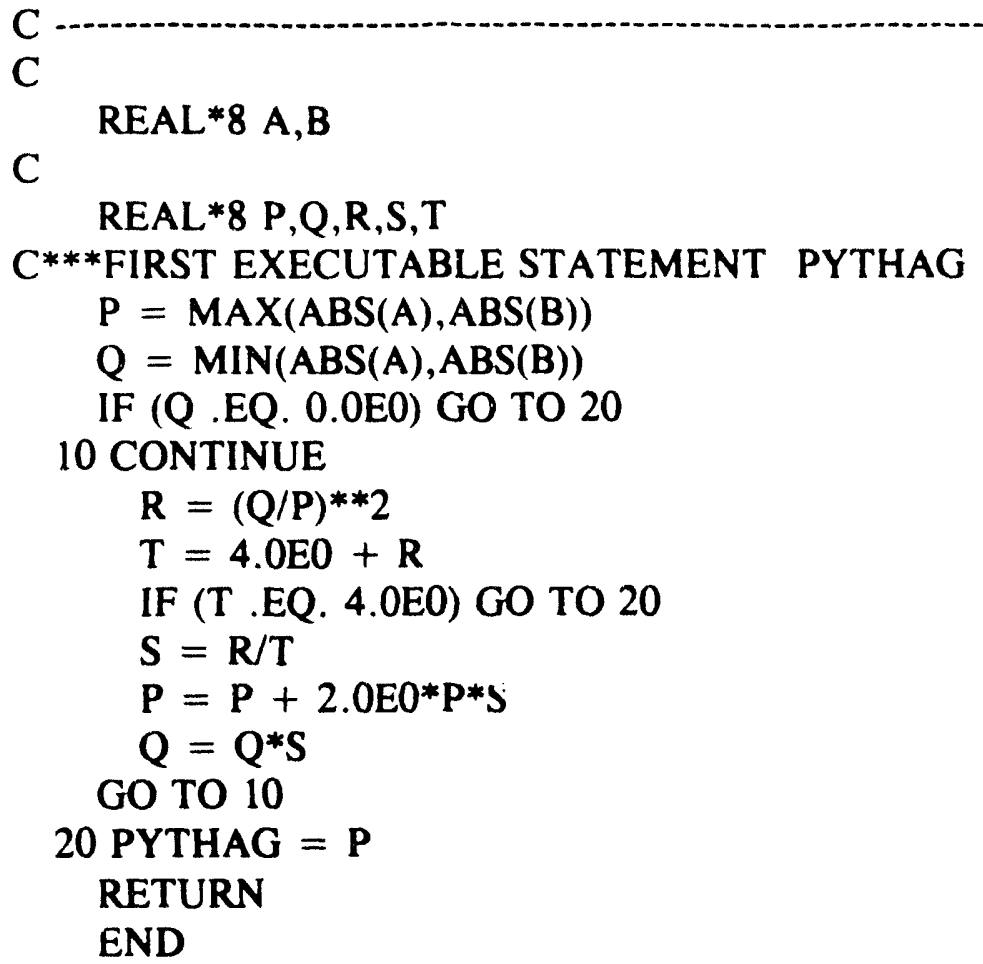

$\mathrm{C} * * * * * * * * * * * * * * * * * * * * * * * * * * * * * * * * * * * * * * * * * * * * * * * * * * * * * * * * * * * * * * * * * * * * * * * *)$

REAL*8 FUNCTION erfun( $x$ )

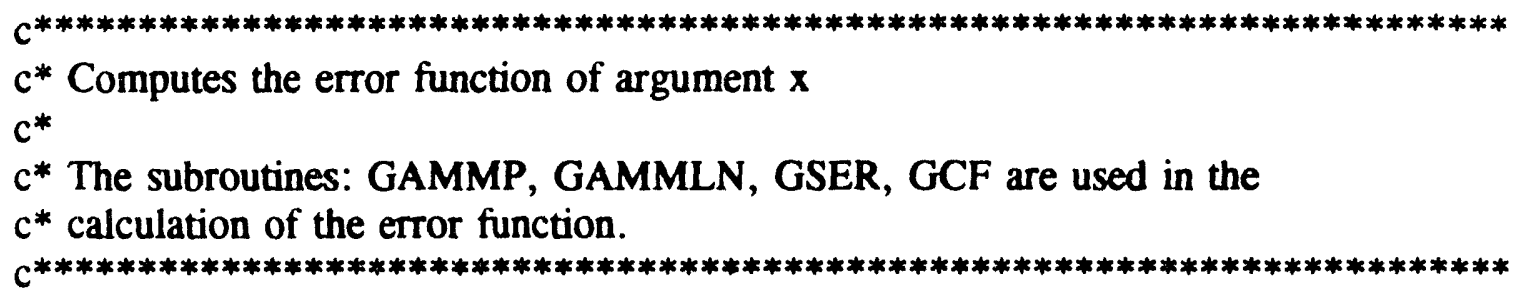

REAL*8 a,x,gammp

$\mathrm{a}=0.5$

IF (x .LT. 0) THEN

erfun $=-\operatorname{gammp}\left(a, x^{* * 2}\right)$

ELSE

erfun $=$ gammp $\left(a, x^{* * 2}\right)$

ENDIF

return

end

REAL*8 FUNCTION gammp $(a, x)$ 
REAL*8 gamser, $a, x$, gln, gammcf

IF ((x .LT. 0.0) .OR. (a .LE. 0.0)) PAUSE

IF ( $x$.LT. $(a+1.0))$ THEN

call gser(gamser,a, $x, g l n$ )

gammp = gamser

ELSE

call gcf(gammcf,a,x,gln)

gammp $=1.0$-gammcf

ENDIF

return

end

REAL*8 FUNCTION gammln(xx)

REAL $* 8$ cof(6),stp, half, one, fpf, $x$, tmp,ser, $x x$

DATA half,one,fpf/0.5, 1.0,5.5/



SUBROUTINE gser(gamser,a, $x$, gln)

REAL*8 gamser,a, $x, g l n$, gammln,ap,sum,del PARAMETER $($ itmax $=100$,eps $=3.0 \mathrm{e}-7)$ 




REAL*8 gammcf,a, $x, g l n, g o l d, a 0, a 1, b o, b 1$, fac, an, ana,anf, $g$ REAL*8 gammln

PARAMETER $($ itmax $=100$, eps $=3.0 \mathrm{e}-7)$

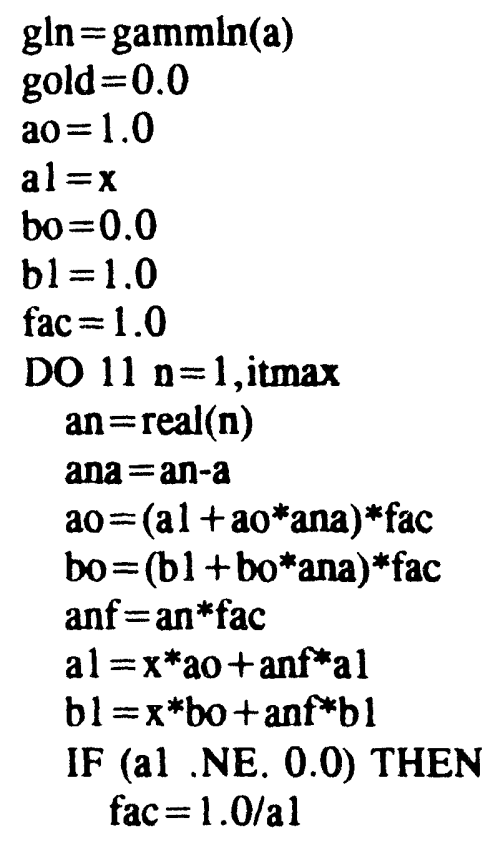




$$
\mathrm{g}=\mathrm{bl} \mathbf{f}_{\mathrm{fac}}
$$

IF(ABS((g-gold)/g) .LT. eps) GOTO 1 gold $=\mathrm{g}$

ENDIF

11 CONTINUE

PAUSE 'A too large, itmax too small'

1 gammcf $=\exp (-x+a * a \log (x)-g l n) * g$

return

end 
References

1. Digest of Topical Meeting on High Energy Density Physics with Subpicosecond Laser Pulses, 1989 (Optical Society of America, Washington, DC, 1989).

2. G. Mourou, and D. Umstadter, Phys. Fluids B 4, 2315 (1992).

3. L. Spitzer, Jr., Physics of Fully Ionized Gases (Interscience, New York, 1962).

4. J.A. Stamper, K. Papadopoulos, R.N. Sudan, S.O. Dean, E.A. McLean, and J.M. Dawson, Phys. Rev. Lett. 26, 1012 (1971).

5. J.A. Stamper, Laser and Particle Beams 9, 841 (1991).

6. M.K. Srivastava, S.V. Lawanda, M. Khan, C. Das, and B. Chakraborty, Phys. Fluids B 4, 4086 (1992).

7. I.H. Hutchinson, Principles of Plasma Diagnostics (Cambridge University Press, New York, 1987).

8. J.D. Jackson, Classical Electrodynamics, 2nd. Ed. (John Wiley \& Sons, New York, 1975).

9. F.F. Chen, Introduction to Plasma Physics and Controlled Fusion, Vol. 1, 2nd. ed. (Plenum Press, New York, 1984).

10. S.I. Braginskii, in Reviews of Plasma Physics, edited by M.A. Leontovich (Consultants Bureau, New York, 1965), Vol. 1, p. 249.

11. E.M. Epperlein and M.G. Haines, Phys. Fluids 29, 1029 (1986).

12. N.K. Winsor, and D.A. Tidman, Phys. Rev. Lett. 31, 1044 (1973).

13. C.E. Max, Laser-plasma Interaction Course 2: Physics of the Coronal Plasma in Laser Fusion Targets, edited by R. Balian and J.C. Adam (North-Holland Publishing Company, 1982).

14. W.L. Kruer, The Physics of Laser Plasma Interactions (Addison-Wesley, Redwood City, CA, 1988).

15. G. Bekefi, C. Deutsch, and B. Yaakobi, in Principles of Laser Plasmas, edited by George Bekefi (John Wiley \& Sons, New York, 1976), Chap. 13, pp. 549-669.

16. T.P. Hughes, Plasmas and Laser Light (John Wiley \& Sons, New York, 1975).

17. D.V. Giovanielli, J.F. Kephart, and A.H. Williams, J. Appl. Phys. 47, 2907 (1976). 
18. R.E. Kidder, and J.W. Zink, Nucl. Fusion 12, 325 (1972).

19. K.A. Brueckner, and S. Jorna, Reviews of Modern Physics 46, 325 (1974).

20. R.S. Craxton, R.L. McCrory, and J.M. Soures, Scientific American 255, 68 (1986).

21. S. Nakai, Nuclear Fusion 30, 1779 (1990).

22. A.W. Ehler, and W. Linlor, J. Appl. Phys. 44, 4229 (1973).

23. J.N. Olsen, G.W. Kuswa, and E.D. Jones, J. Appl. Phys. 44, 2275 (1973).

24. S.J. Gitomer, R.D. Jones, F. Begay, A.W. Ehler, J.F. Kephart, and R. Kristal, Phys. Fluids 29, 2679 (1986).

25. M. Nakai, et. al., Rev. Sci. Instrum. 61, 3235 (1990).

26. M.A. Russotto, and R.L. Kremens, Rev. Sci. Instrum. 61, 3125 (1990).

27. H.R. Griem, Phys. Fluids B 4, 2346 (1992).

28. O. Willi, G. Kiehn, J. Edwards, V. Barrow, R.A. Smith, J. Wark, and E. Turcu, Europhys. Lett. 10, 141 (1989).

29. J.T. Knudtson, W.B. Green, and D.G. Sutton, J. Appl. Phys. 61, 4771 (1987).

30. R.W. Lee, D.L. Matthews, and L. Koppel, J. Appl. Phys. 54, 4909 (1983).

31. J.N. Olsen, and C.W. Mendel, Jr., J. Appl. Phys. 46, 4407 (1975).

32. W. Seka, et. al., Phys. Fluids B 4, 432 (1992).

33. R.S. Marjoribanks, M.C. Richardson, J. Delettrez, S. Letzring, W. Seka, and D.M. Villeneuve, Optics Comm. 44, 113 (1982).

34. E.A. McLean, J.A. Stamper, C.K. Manka, H.R. Griem, D.W. Droemer, and B.H. Ripin, Phys. Fluids 27, 1327 (1984).

35. J.C. Kieffer, J.P. Matte, H. Pépin, M. Chaker, Y. Beaudoin, T.W. Johnston, C.Y. Chien, S. Coe, G. Mourou, and J. Dubau, Phys. Rev. Lett. 68, 480 (1992).

36. J.F. Kephart, R.P. Godwin, and G.H. McCall, Appl. Phys. Lett. 25, 108 (1974).

37. V.W. Slivinsky, H.N. Kornblum, and D.H. Shay, J. Appl. Phys. 46, 1973 (1975).

38. B.H. Ripin, et. al., Phys. Rev. Lett. 34, 1313 (1975). 
39. N.M. Ceglio, and J.T. Larsen, Phys. Rev. Lett. 44, 579 (1980).

40. O.L. Landen, E.M. Campbell, and M.D. Perry, Optics Comm. 63, 253 (1987).

41. P.E. Young, J.D. Hares, J.D. Kilkenny, D.W. Phillion, and E.M. Campbell, Rev. Sci. Instrum. 59, 1457 (1988).

42. J.D. Kilkenny, P. Bell, R. Hanks, G. Power, R.E. Turner, and J. Wiedwald, Rev. Sci. Instrum. 59, 1793 (1988).

43. D.G. Sterns, J.D. Wiedwald, B.M. Cook, R.L. Hanks, and O.L. Landen, Rev. Sci. Instrum. 60, 363 (1989).

44. M. Katayama, M. Nakai, T. Yamanaka, Y. Izawa, and S. Nakai, Rev. Sci. Instrum. 62, 124 (1991).

45. F.D. Seward, and T.M. Palmieri, Rev. Sci. Instrum. 46, 204 (1975).

46. M. Nakai, et. al., Rev. Sci. Instrum. 61, 2783 (1990).

47. D.T. Attwood, and L.W. Coleman, Applied Physics Letters 24, 408 (1974).

48. D.T. Attwood, L.W. Coleman, and D.W. Sweeney, Applied Physics Letters 26, 616 (1975).

49. J.F. Reintjes, T.N. Lee, R.C. Eckardt, and R.A. Andrews, J. of Applied Physics 47 , 4457 (1976).

50. P.E. Young, Phys. Fluids B 3, 2331 (1991).

51. D.T. Attwood, D.W. Sweeney, J.M. Auerbach, and P.H.Y. Lee, Phys. Rev. Lett. 40, 184 (1978).

52. G.E. Busch, C.L. Shepard, L.D. Siebert, and J.A. Tarvin, Rev. Sci. Instrum. 56, 879 (1985).

53. S.E. Coe, T. Afshar-rad, and O. Willi, Optics Comm. 73, 299 (1989).

54. S.E. Coe, T. Afshar-rad, and O. Willi, Europhys. Lett. 13, 251 (1990).

55. O. Willi, T. Afshar-rad, S. Coe, A. Giulietti, Phys. Fluids B 2, 1318 (1990).

56. T. Afshar-rad, L.A. Gizzi, M. Desselberger, F. Khattak, O. Willi, and A. Giulietti, 68, 942 (1992).

57. J. Bruneau, et. al., Phys. Rev. Lett. 65, 1435 (1990). 
58. J.P. Geindre, C. Chenais-Popovics, P. Audebert, C.A. Back, and J.C. Gauthier, Phys. Rev. A 43, 3202 (1991).

59. D.M. O'Neill, C.L.S. Lewis, D. Neely, S.J. Davidson, S.J. Rose, and R.W. Lee, Phys. Rev. A 44, 2641 (1991).

60. R.R. Whitlock, M.H. Emery, J.A. Stamper, E.A. McLean, S.P. Obenschain, and M.C. Peckerar, Phys. Rev. Lett. 52, 819 (1984).

61. J. Grun, M.H. Emery, S. Kacenjar, C.B. Opal, E.A. McLean, S.P. Obenschain, B.H. Ripin, and A. Schmitt, Phys. Rev. Lett. 53, 1352 (1984).

62. M. Desselberger, O. Willi, M. Savage, and M.J. Lamb, Phys. Rev. Lett. 65, 2997 (1990).

63. B.A. Remington, S.W. Haan, S.G. Glendinning, J.D. Kilkenny, D.H. Munro, and R.J. Wallace, Phys. Rev. Lett. 67, 3259 (1991).

64. B.A. Remington, S.W. Haan, S.G. Glendinning, J.D. Kilkenny, D.H. Munro, and R.J. Wallace, Phys. Fluids B 4, 967 (1992).

65. M. Desselberger, T. Afshar-rad, F. Khattak, S. Viana, and O. Willi, Phys. Rev. Lett. 68, 1539 (1992).

66. O.L. Landen, D.G. Stearns, and E.M. Campbell, Phys. Rev. Lett. 63, 1475 (1989).

67. J.F. Drake, P.K. Kaw, Y.C. Lee, G. Schmidt, C.S. Liu, and M.N. Rosenbluth, Phys. Fluids 17, 778 (1974).

68. C.S. Liu, M.N. Rosenbluth, and R.B. White, Phys. Fluids 17, 1211 (1974).

69. D.W. Forslund, J.M. Kindel, and E.L. Lindman, Phys. Fluids 18, 1002 (1975).

70. A. Simon, R.W. Short, E.A. Williams, and T. Dewandre, Phys. Fluids 26, 3107 (1983).

71. R.P. Drake, E.A. Williams, P.E. Young, K. Estabrook, W.L. Kruer, H.A. Baldis, and T.W. Johnston, Phys. Rev. A 39, 3536 (1989).

72. R.P. Drake, E.A. Williams, P.E. Young, K. Estabrook, W.L. Kruer, D.S. Montgomery, H.A. Baldis, and T.W. Johnston, Phys. Fluids B 1, 2217 (1989).

73. S.H. Batha, D.D. Meyerhofer, A. Simon, and R.P. Drake, Phys. Fluids B 3, 448 (1991). 
74. C.B. Darrow, R.P. Drake, D.S. Montgomery, P.E. Young, K. Estabrook, and W.L. Kruer, Phys. Fluids B 3, 1473 (1991).

75. R.P. Drake, H.A. Baldis, R.L. Berger, W.L. Kruer, E.A. Williams, K. Estabrook, T.W. Johnston, and P.E. Young, Phys. Rev. Lett. 64, 423 (1990).

76. P.E. Young, K.G. Estabrook, W.L. Kruer, E.A. Williams, P.J. Wegner, R.P. Drake, H.A. Baldis, and T.W. Johnston, Phys. Fluids B 2, 1907 (1990).

77. R.P. Drake, R.E. Turner, B.F. Lasinski, E.M. Campbell, W.L. Kruer, E.A. Williams, and R.L. Kauffman, Phys. Fluids B 1, 1295 (1989).

78. J.A. Stamper, R.H. Lehmberg, A. Schmitt, M.J. Herbst, F.C. Young, J.H. Gardner, and S.P. Obenschain, Phys. Fluids 28, 2563 (1985).

79. K. Mizuno, P.E. Young, W. Seka, R. Bahr, J.S. De Groot, R.P. Drake, and K.G. Estabrook, Phys. Rev. Lett. 65, 428 (1990).

80. K. Mizuno, R.P. Drake, P.E. Young, R. Bahr, W. Seka, and K.G. Estabrook, Phys. Fluids B 3, 1983 (1991).

81. W. Seka, R.E. Bahr, R.W. Short, A. Simon, and R.S. Craxton, Phys. Fluids B 4, 2232 (1992).

82. B. La Fontaine, D.M. Villeneuve, H.A. Baldis, R.P. Drake, and K. Estabrook, Phys. Rev. Lett. 68, 484 (1992).

83. K.L. Bowles, Phys. Rev. Lett. 1, 454 (1958).

84. K.L. Bowles, Journal of Research of the National Bureau of Standards 65D, 1 (1961).

85. G. Fiocco and E. Thompson, Phys. Rev. Lett. 10, 89 (1963).

86. E. Fünfer, B. Kronast, H.-J. Kunze, Phys. Lett. 5, 125 (1963).

87. H.J. Kunze, E. Fünfer, B. Kronast, and W.H. Kegel, Phys. Lett. 11, 42 (1964).

88. A.W. DeSilva, D.E. Evans, and M.J. Forrest, Nature 203, 1321 (1964).

89. U. Ascoli-Bartoli, J. Katzenstein, and L. Lovisetto, Nature 204, 672 (1964); 207, 63 (1965).

90. J.E. Bernard, H.A. Baldis, and D.M. Villeneuve, Phys. Fluids 30, 3616 (1987).

91. O.L. Landen and R.J. Winfield, Phys. Rev. Lett. 54, 1660 (1985). 
92. D.M. Villeneuve, C.J. Walsh, and H.A. Baldis, Phys. Fluids 28, 1591 (1985).

93. D.M. Villeneuve, J.E. Bernard, and H.A. Baldis, Phys. Fluids 30, 3832 (1987).

94. D. Umstadter, R. Williams, C. Clayton, and C. Joshi, Phys. Rev. Lett. 59, 292 (1987).

95. G. McIntosh, J. Meyer, and Z. Yazhou, Phys. Fluids 29, 3451 (1986).

96. G. McIntosh, H. Houtman, and J. Meyer, Phys. Rev. Lett. 57, 337 (1986).

97. C.J. Walsh and H.A. Baldis, Phys. Rev. Lett. 48, 1483 (1982).

98. C.E. Clayton, C. Joshi, and F.F. Chen, Phys. Rev. Lett. 51, 1656 (1983).

99. J.E. Bernard and J. Meyer, Phys. Fluids 29, 2313 (1986); 28, 2618 (1985).

100. Y. Al-Shiraida and A.A. Offenberger, Phys. Fluids 30, 593 (1987); Phys. Rev. Lett.

52, 283 (1984).

101. R. Giles and A.A. Offenberger, Phys. Rev. Lett. 50, 421 (1983).

102. H.A. Baldis, J.C. Samson, and P.B. Corkum, Phys. Rev. Lett. 41, 1719 (1978).

103. H.A. Baldis and C.J. Walsh, Phys. Rev. Lett. 47, 1658 (1981); Phys. Fluids 26, 1364 (1983).

104. J. Meyer and H. Houtman, Phys. Rev. Lett. 53, 1344 (1984).

105. D. Umstadter, W.B. Mori, and C. Joshi, Phys. Fluids B 1, 183 (1989).

106. B. Amini and F.F. Chen, Phys. Rev. Lett. 53, 1441 (1984).

107. C.E. Clayton, C. Joshi, C. Darrow, and D. Umstadter, Phys. Rev. Lett. 54, 2343 (1985).

108. G.B. Zimmerman and W.L. Kruer, Comments Plasma Phys. Controlled Fusion 2, 51 (1975).

109. J. Sheffield, Plasma Scattering of Electromagnetic Radiation (Academic Press, New York, 1975).

110. L.D. Landau and E.M. Lifshitz, Statistical Physics, 2nd ed. (J.B. Sykes and M.J. Kearsley, transls.) (Addison-Wesley, Reading, Mass. 1969).

111. H.B. Callen and T.A. Welton, Phys. Rev. 83, 34 (1951). 
112. R. Kubo, J. Phys. Soc. Japan 12, 570 (1957).

113. A.G. Sitenko, Electromagnetic Fluctuations in Plasma (M.D. Friedman, transl.) (Academic, New York, 1967).

114. S. Ichimaru, Basic Principles of Plasma Physics (W.A. Benjamin, Inc., Reading, Mass., 1973).

115. J.A. Fejer, Can. J. Phys. 38, 1114 (1960).

116. J. Renau, J. Geophys. Res. 65, 3631 (1960).

117. J.P. Dougherty and D.T. Farley, Proc. Roy. Soc. Ser. A. 259, 79 (1960).

118. E.E. Salpeter, Phys. Rev. 120, 1528 (1960).

119. D.E. Evans and J. Katzenstein, Rep. Prog. Phys. 32, 207 (1969).

120. K.L. Bowles, Advan. Electron. Electron Phys. 19, 55 (1964).

121. L.D. Landau, J. Phys. (USSR) 10, 25 (1946).

122. B.D. Fried and S.D. Conte, The Plasma Dispersion Function (Academic Press, New York, 1961).

123. E.C. Taylor and G.G. Comisar, Phys. Rev. 132, 2379 (1963).

124. J.P. Dougherty, Phys. Fluids 7, 1788 (1964).

125. M. Grewal, Phys. Rev. A 134, 86 (1964).

126. D.F. DuBois and V. Gilinski, Phys. Rev. A 133, 1308 (1964); 1317 (1964).

127. Y.Q. Zhang and A.W. DeSilva, Phys. Rev. A 44, 3841 (1991).

128. A.E. Siegman, Lasers (University Science Books, Mill Valley, CA, 1986).

129. G. Giuliani, Y.K. Park, and R.L. Byer, Opt. Lett. 5, 491 (1980).

130. S. DeSilvestri, P. Laporta, V. Magni, and O. Svelto, Opt. Lett. 12, 84 (1987).

131. K.J. Snell, N. McCarthy, and M. Piche, Opt. Commun. 65, 377 (1988).

132. D.A. McQuarrie, Statistical Mechanics (Harper \& Row, New York, 1976).

133. H.J. Kunze, in Plasma Diagnostics, edited by W. Lochte-Holtgreven (Wiley, New York, 1968), Chap. 9, pp. 550-611. 
134. J.I. Steinfeld, Molecules and Radiation - An Introduction to Modern Molecular Spectroscopy (MIT Press, Cambridge, MA, 1978).

135. Landolt-Bornstein Zahlenwerte und Funktionen aus Physik, Chemie, Astronomie, Geophsik, und Technik (Springer-Verlag, Berlin, 1962), Vol.2, Pt.8.

136. D. Colombant and G.F. Tonon, J. Appl. Phys. 44, 3524 (1973).

137. R.P. McWhirter, in Plasma Diagnostic Techniques, edited by R.H. Huddlestone and S.L. Leonard (Academic Press, New York, 1965), p. 201-261.

138. R.L. Kelley, J. Phys. Chem. Ref. Data 16, Suppl. 1, 218 (1987).

139. H.R. Griem, Plasma Spectroscopy (McGraw-Hill, New York, 1964).

140. J.W. Shearer and W.S. Barnes, in Laser Interaction and Related Plasma Phenomena edited by H.J. Schwarz and H. Hora (Plenum Press, New York, 1971), Vol. 1, pp. 307-337.

141. Y.T. Lee, J. Quant. Spectrosc. Radiat. Transfer 38, 131 (1987).

142. See National Technical Information Service Document No. DE87011009 (Lawrence Livermore National Laboratory Report No. UCRL-5002185 by Y.T. Lee, D.S. Bailey, and G.B. Zimmermann, pp.2-81, 1986). Copies may be obtained from the National Technical Information Service, Springfield, Virginia, 22161. The price is $\$ 73.00$ plus a $\$ 3.00$ handling fee. All orders must be prepaid.

143. J.S. De Groot, S.M. Cameron, K. Mizuno, K.G. Estabrook, R.P. Drake, W.L. Kruer, and P.E. Young, Phys. Fluids B 3, 1241 (1991).

144. J.S. De Groot, K.G. Estabrook, W.L. Kruer, R.P. Drake, K. Mizuno, and S.M. Cameron, Phys. Fluids B 4, 701 (1992).

145. D.L. Book, NRL Plasma Formulary (NRL Publication 0084-4040) (Naval Research Laboratory, Washington, D.C., 20375-5000, 1987).

146. P. Monchicourt and P.A. Holstein, Phys. Fluids 23, 1475 (1980).

147. L. Spitzer and R. Härm, Physical Rev. 89, 977 (1953).

148. Z. Chang and J.D. Callen, Phys. Fluids B 4, 1167 (1992).

149. R.L. Liboff, Kinetic Theory: Classical, Quantum, and Relativistic Descriptions (Prentice Hall, N.J., 1990).

150. M. Ono and R.M. Kulsrud, Phys. Fluids 18, 1287 (1975). 
74. C.B. Darrow, R.P. Drake, D.S. Montgomery, P.E. Young, K. Estabrook, and W.L. Kruer, Phys. Fluids B 3, 1473 (1991).

75. R.P. Drake, H.A. Baldis, R.L. Berger, W.L. Kruer, E.A. Williams, K. Estabrrok, T.W. Johnston, and P.E. Young, Phys. Rev. Lett. 64, 423 (1990).

76. P.E. Young, K.G. Estabrook, W.L. Kruer, E.A. Williams, P.J. Wegner, R.P. Drake, H.A. Baldis, and T.W. Johnston, Phys. Fluids B 2, 1907 (1990).

77. R.P. Drake, R.E. Turner, B.F. Lasinski, E.M. Campbell, W.L. Kruer, E.A. Williams, and R.L. Kauffman, Phys. Fluids B 1, 1295 (1989).

78. J.A. Stamper, R.H. Lehmberg, A. Schmitt, M.J. Herbst, F.C. Young, J.H. Gardner, and S.P. Obenschain, Phys. Fluids 28, 2563 (1985).

79. K. Mizuno, P.E. Young, W. Seka, R. Bahr, J.S. De Groot, R.P. Drake, and K.G. Estabrook, Phys. Rev. Lett. 65, 428 (1990).

80. K. Mizuno, R.P. Drake, P.E. Young, R. Bahr, W. Seka, and K.G. Estabrook, Phys. Fluids B 3, 1983 (1991).

81. W. Seka, R.E. Bahr, R.W. Short, A. Simon, and R.S. Craxton, Phys. Fluids B 4, 2232 (1992).

82. B. La Fontaine, D.M. Villeneuve, H.A. Baldis, R.P. Drake, and K. Estabrook, Phys. Rev. Lett. 68, 484 (1992).

83. K.L. Bowles, Phys. Rev. Lett. 1, 454 (1958).

84. K.L. Bowles, Journal of Research of the National Bureau of Standards 65D, 1 (1961).

85. G. Fiocco and E. Thompson, Phys. Rev. Lett. 10, 89 (1963).

86. E. Fünfer, B. Kronast, H.-J. Kunze, Phys. Lett. 5, 125 (1963).

87. H.J. Kunze, E. Fünfer, B. Kronast, and W.H. Kegel, Phys. Lett. 11, 42 (1964).

88. A.W. DeSilva, D.E. Evans, and M.J. Forrest, Nature 203, 1321 (1964).

89. U. Ascoli-Bartoli, J. Katzenstein, and L. Lovisetto, Nature 204, 672 (1964); 207, 63 (1965).

90. J.E. Bernard, H.A. Baldis, and D.M. Villeneuve, Phys. Fluids 30, 3616 (1987).

91. O.L. Landen and R.J. Winfield, Phys. Rev. Lett. 54, 1660 (1985). 
92. D.M. Villeneuve, C.J. Walsh, and H.A. Baldis, Phys. Fluids 28, 1591 (1985).

93. D.M. Villeneuve, J.E. Bernard, and H.A. Baldis, Phys. Fluids 30, 3832 (1987).

94. D. Umstadter, R. Williams, C. Clayton, and C. Joshi, Phys. Rev. Lett. 59, 292 (1987).

95. G. McIntosh, J. Meyer, and Z. Yazhou, Phys. Fluids 29, 3451 (1986).

96. G. McIntosh, H. Houtman, and J. Meyer, Phys. Rev. Lett. 57, 337 (1986).

97. C.J. Walsh and H.A. Baldis, Phys. Rev. Lett. 48, 1483 (1982).

98. C.E. Clayton, C. Joshi, and F.F. Chen, Phys. Rev. Lett. 51, 1656 (1983).

99. J.E. Bernard and J. Meyer, Phys. Fluids 29, 2313 (1986); 28, 2618 (1985).

100. Y. Al-Shiraida and A.A. Offenberger, Phys. Fluids 30, 593 (1987); Phys. Rev. Lett.

52,283 (1984).

101. R. Giles and A.A. Offenberger, Phys. Rev. Lett. 50, 421 (1983).

102. H.A. Baldis, J.C. Samson, and P.B. Corkum, Phys. Rev. Lett. 41, 1719 (1978).

103. H.A. Baldis and C.J. Walsh, Phys. Rev. Lett. 47, 1658 (1981); Phys. Fluids 26, 1364 (1983).

104. J. Meyer and H. Houtman, Phys. Rev. Lett. 53, 1344 (1984).

105. D. Umstadter, W.B. Mori, and C. Joshi, Phys. Fluids B 1, 183 (1989).

106. B. Amini and F.F. Chen, Phys. Rev. Lett. 53, 1441 (1984).

107. C.E. Clayton, C. Joshi, C. Darrow, and D. Umstadter, Phys. Rev. Lett. 54, 2343 (1985).

108. G.B. Zimmerman and W.L. Kruer, Comments Plasma Phys. Controlled Fusion 2, 51 (1975).

109. J. Sheffield, Plasma Scattering of Electromagnetic Radiation (Academic Press, New York, 1975).

110. L.D. Landau and E.M. Lifshitz, Statistical Physics, 2nd ed. (J.B. Sykes and M.J. Kearsley, transls.) (Addison-Wesley, Reading, Mass. 1969).

111. H.B. Callen and T.A. Welton, Phys. Rev. 83, 34 (1951). 
112. R. Kubo, J. Phys. Soc. Japan 12, 570 (1957).

113. A.G. Sitenko, Electromagnetic Fluctuations in Plasma (M.D. Friedman, transl.) (Academic, New York, 1967).

114. S. Ichimaru, Basic Principles of Plasma Physics (W.A. Benjamin, Inc., Reading, Mass., 1973).

115. J.A. Fejer, Can. J. Phys. 38, 1114 (1960).

116. J. Renau, J. Geophys. Res. 65, 3631 (1960).

117. J.P. Dougherty and D.T. Farley, Proc. Roy. Soc. Ser. A. 259, 79 (1960).

118. E.E. Salpeter, Phys. Rev. 120, 1528 (1960).

119. D.E. Evans and J. Katzenstein, Rep. Prog. Phys. 32, 207 (1969).

120. K.L. Bowles, Advan. Electron. Electron Phys. 19, 55 (1964).

121. L.D. Landau, J. Phys. (USSR) 10, 25 (1946).

122. B.D. Fried and S.D. Conte, The Plasma Dispersion Function (Academic Press, New York, 1961).

123. E.C. Taylor and G.G. Comisar, Phys. Rev. 132, 2379 (1963).

124. J.P. Dougherty, Phys. Fluids 7, 1788 (1964).

125. M. Grewal, Phys. Rev. A 134, 86 (1964).

126. D.F. DuBois and V. Gilinski, Phys. Rev. A 133, 1308 (1964); 1317 (1964).

127. Y.Q. Zhang and A.W. DeSilva, Phys. Rev. A 44, 3841 (1991).

128. A.E. Siegman, Lasers (University Science Books, Mill Valley, CA, 1986).

129. G. Giuliani, Y.K. Park, and R.L. Byer, Opt. Lett. 5, 491 (1980).

130. S. DeSilvestri, P. Laporta, V. Magni, and O. Svelto, Opt. Lett. 12, 84 (1987).

131. K.J. Snell, N. McCarthy, and M. Piche, Opt. Commun. 65, 377 (1988).

132. D.A. McQuarrie, Statistical Mechanics (Harper \& Row, New York, 1976).

133. H.J. Kunze, in Plasma Diagnostics, edited by W. Lochte-Holtgreven (Wiley, New York, 1968), Chap. 9, pp. 550-611. 
134. J.I. Steinfeld, Molecules and Radiation - An Introduction to Modern Molecular Spectroscopy (MIT Press, Cambridge, MA, 1978).

135. Landolt-Bomstein Zahlenwerte und Funkrionen aus Physik, Chemie, Astronomie, Geophsik, und Technik (Springer-Verlag, Berlin, 1962), Vol.2, Pt.8.

136. D. Colombant and G.F. Tonon, J. Appl. Phys. 44, 3524 (1973).

137. R.P. McWhirter, in Plasma Diagnostic Techniques, edited by R.H. Huddlestone and S.L. Leonard (Academic Press, New York, 1965), p. 201-261.

138. R.L. Kelley, J. Phys. Chem. Ref. Data 16, Suppl. 1, 218 (1987).

139. H.R. Griem, Plasma Spectroscopy (McGraw-Hill, New York, 1964).

140. J.W. Shearer and W.S. Barnes, in Laser Interaction and Related Plasma Phenomena edited by H.J. Schwarz and H. Hora (Plenum Press, New York, 1971), Vol. 1, pp. 307-337.

141. Y.T. Lee, J. Quant. Spectrosc. Radiat. Transfer 38, 131 (1987).

142. See National Technical Information Service Document No. DE870110n9 (Lawrence Livermore National Laboratory Report No. UCRL-5002185 by Y.T. Lee, D.S. Bailey, and G.B. Zimmermann, pp.2-81, 1986). Copies may be obtained from the National Technical Information Service, Springfield, Virginia, 22161. The price is $\$ 73.00$ plus a $\$ 3.00$ handling fee. All orders must be prepaid.

143. J.S. De Groot, S.M. Cameron, K. Mizuno, K.G. Estabrook, R.P. Drake, W.L. Kruer, and P.E. Young, Phys. Fluids B 3, 1241 (1991).

144. J.S. De Groot, K.G. Estabrook, W.L. Kruer, R.P. Drake, K. Mizuno, and S.M. Cameron, Phys. Fluids B 4, 701 (1992).

145. D.L. Book, NRL Plasma Formulary (NRL Publication 0084-4040) (Naval Research Laboratory, Washington, D.C., 20375-5000, 1987).

146. P. Monchicourt and P.A. Holstein, Fhys. Fluids 23, 1475 (1980).

147. L. Spitzer and R. Härm, Physical Rev. 89, 977 (1953).

148. Z. Chang and J.D. Callen, Phys. Fluids B 4, 1167 (1992).

149. R.L. Liboff, Kinetic Theory: Classical, Quantum, and Relativistic Descriptions (Prentice Hall, N.J., 1990).

150. M. Ono and R.M. Kulsrud, Phys. Fluids 18, 1287 (1975). 
151. C.J. Randall, Phys. Fluids 25, 2231 (1982).

152. I.P. Shkarofsky, T.W. Johnston, M.P. Bachynski, The Particle Kinetics of Plasmas (Addison-Wesley Publishing Company, Inc., 1966).

153. M.N. Rosenbluth, W.M. MacDonald, and D.L. Judd, Phys. Rev. 107, 1 (1957).

154. E.M. Epperlein, R.W. Short, and A. Simon, Phys. Rev. Lett. 69, 1765 (1992).

155. H.F. Talbot, Phil. Mag. (Third Series) 9, 401 (1836).

156. M.D. Tracy, J.S. De Groot, K.G. Estabrook, and S.M. Cameron, Phys. Fluids B 4, 1576 (1992).

157. S.M. Cameron, M.D. Tracy, K.G. Estabrook, and J.S. De Groot, Rev. Sci. Instrum. 63, 5259 (1992).

158. M.D. Tracy, E.A. Williams, K.G. Estabrook, J.S. De Groot, and S.M. Cameron, 'Eigenvalue solution of the ion-collisional effects on ion-acoustic and entropy waves' submitted to Phys. Fluids B (Jan. 1993). 

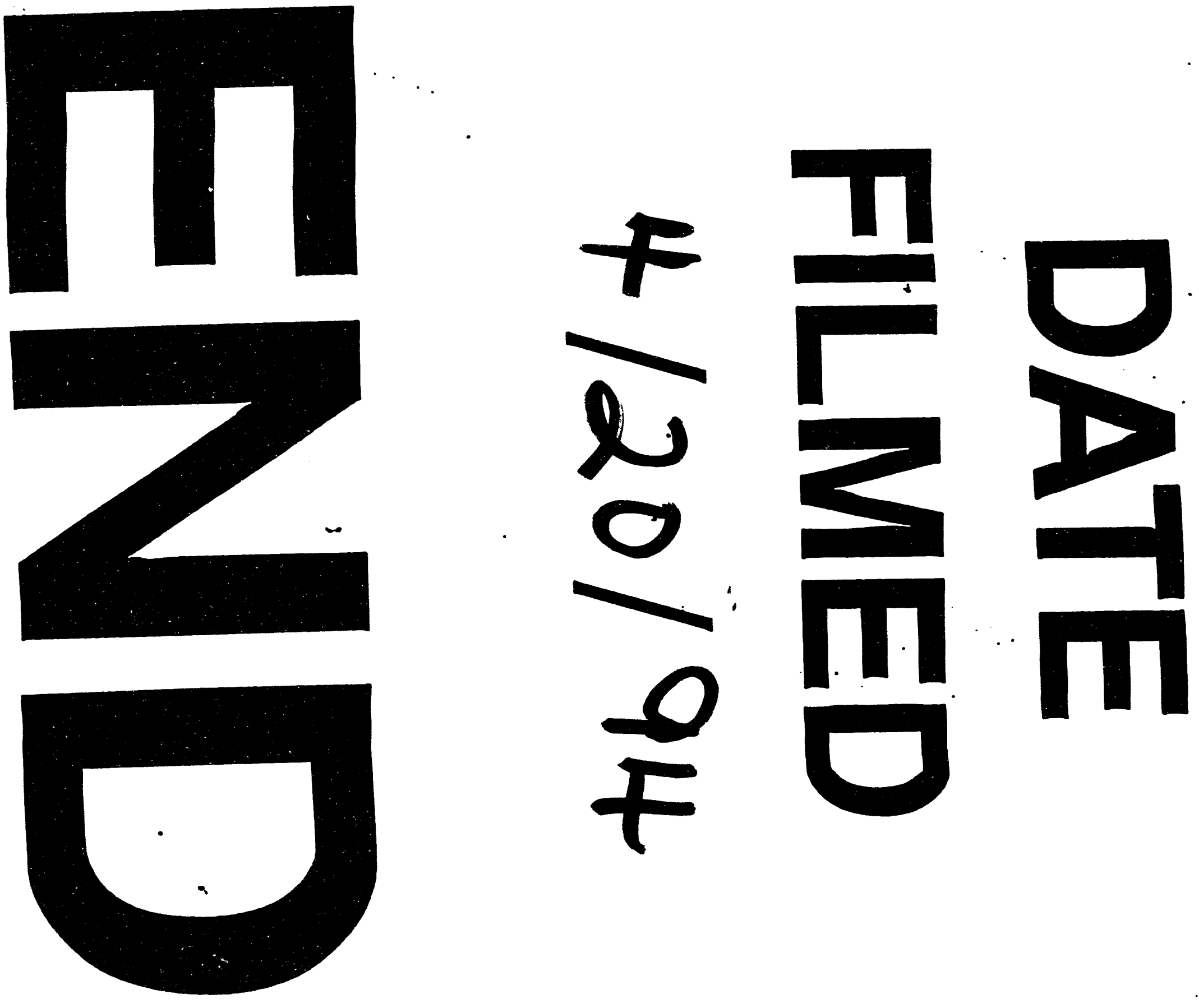

F 
